FROZEN OUT: AUDIENCES, AFFECT AND WOMEN'S HOCKEY

\author{
by \\ Donna H. Gall \\ Bachelor of Arts (Honours), Queen's University, 1989 \\ Master of Philosophy, University of Glasgow, 1995
}

\author{
A dissertation \\ presented to Ryerson University and York University \\ in partial fulfillment of the \\ requirements for the degree of \\ Doctor of Philosophy \\ in the joint program of \\ Communication and Culture
}

Toronto, Ontario, Canada, 2019

(C) Donna H. Gall 2019 


\section{AUTHOR'S DECLARATION FOR ELECTRONIC SUBMISSION OF A DISSERTATION}

I hereby declare that I am the sole author of this dissertation. This is a true copy of the dissertation, including any required final revisions, as accepted by my examiners.

I authorize Ryerson University to lend this dissertation to other institutions or individuals for the purpose of scholarly research.

I further authorize Ryerson University to reproduce this dissertation by photocopying or other means, in total or in part, at the request of other institutions or individuals for the purpose of scholarly research.

I understand that my dissertation may be made electronically available to the public. 


\title{
FROZEN OUT: AUDIENCES, AFFECT AND WOMEN'S HOCKEY
}

Doctor of Philosophy

2019

\author{
Donna H. Gall \\ Communications and Culture Joint Program \\ Ryerson University and York University
}

\begin{abstract}
Every four years, millions of Canadians watch women play hockey during the Olympics. Yet when it comes to regularly scheduled professional games, that audience dramatically decreases. In 2019, low audience numbers led to the closure of the Canadian Women's Hockey League and put the future of professional women's hockey in jeopardy. As with many women's sports, broadcasters argue the cost of production is too great, the value of airtime minutes too high to take the financial risk of televising the women's game without guaranteeing viewers for advertisers. Activists and athletes argue that the audience must be built through broadcaster investment.

While scholars have examined hockey for its representational power to define national and gendered identities, there has been shockingly little research into the hockey audience. This mixed method audience reception study seeks to explore the viewing inconsistencies of the audience for women's hockey. Quantitative results from an online survey $(n=685)$ provided data about viewing habits, perceptions and knowledge. This data informed qualitative focus groups (n $=25$ ) that in turn provided contextualization and reasoning for the quantitative data. Mixed method analysis intersected grounded theory with audience reception, sport media, feminist studies and affect theory to identify a persistent discursive strategy framing women's hockey as
\end{abstract}


"pure" for resisting the crass commercialization, incessant violence and individualistic star system of professional men's hockey. I argue that women's hockey becomes the manifestation of the Canadian myth of hockey; men's hockey as it used to be. As a nostalgic placeholder devoid of context, contemporary women's hockey functions within a double bind; virtuous and elevated yet non-viable as a commercial enterprise. This ensures that the sport remains precarious at best. "Pure" women's hockey also functions as a postfeminist essentializing discourse that solves the gender risk of hockey's hypermasculinity while disavowing women's physically aggressive play and sport media's affective currency. Whereas Olympic women's hockey relies on patriotic pride for audience affective engagement, professional women's hockey is framed by cognitive contradictions, "pure" but commercial, gender normative but transgressive. Confused and disconnected from the game and players, audiences are left unaffected. 


\section{Acknowledgements}

I offer my sincerest gratitude to my supervisor Dr. Jean Bruce from Ryerson University for providing such valuable support, advice, inspiration and empathy during my doctoral journey. Her generosity with her time, attention and expertise ensured my dissertation was thorough, coherent and, most importantly, complete.

I also want to thank my committee members, Dr. Nicole Neverson from Ryerson University and Dr. Natalie Coulter from York University. I am so grateful for their expertise and insight and for offering such unceasing support and warm encouragement.

My academic journey began with Dr. Pamela Robinson from Ryerson University and she has been unflagging in her support and encouragement ever since. I am so very grateful to her for inspiring me to make this enormous life change and for her continued belief in my future as an academic.

My doctoral dissertation was inspired by my documentary Play Fair and so I must acknowledge my producing partners, Chris McIvor and Jeff Peeler. I would also like to acknowledge with sincere gratitude my executive producer Senator Nancy Ruth for also providing me with ongoing support during my academic pursuits. I would also like to acknowledge Beth Atcheson, Donna Dasko, Dr. Sandra Kirby and Sina Zapfe for their advice and encouragement.

I would like to acknowledge the agencies that provided me with the funding that allowed me to complete my doctoral studies in a timely manner. I thank the Roger Fellowship in Communication \& Culture at Ryerson University, the Ontario Graduate Scholarship, the Alfred Bader Fellowship in Memory of Jean Royce Queen's University and Nancy's Very Own Foundation.

Lastly, I would like to acknowledge my family for their generous and ongoing support. I would particularly like to thank my mother, Georgia Gall, and my son William Conway-Gall, for their patience, their encouragement, their humour and their faith in me. 


\section{Dedication}

I dedicate this work to the memory of my father, Dr. Robert Gall, for teaching me that life is work and work is life and it's best when you're passionate about both. 


\section{Table of Contents}

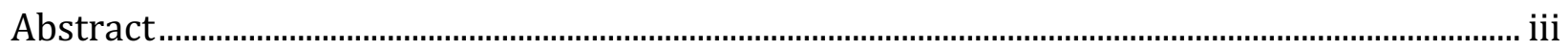

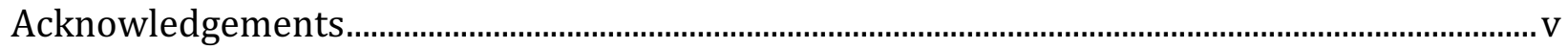

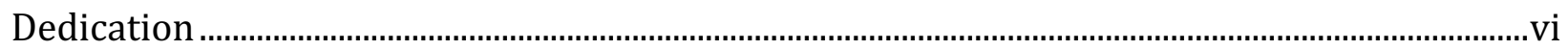

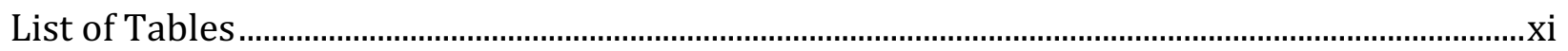

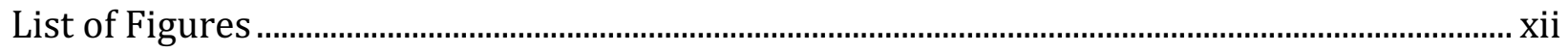

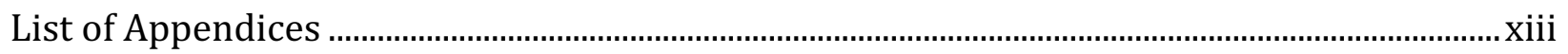

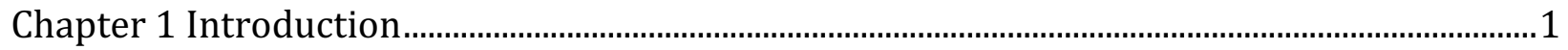

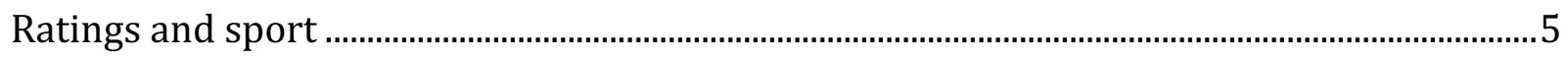

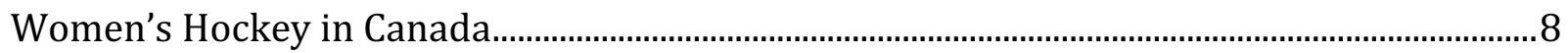

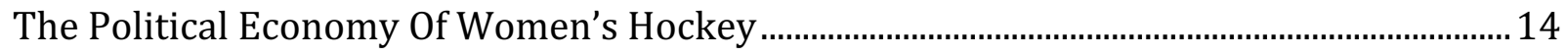

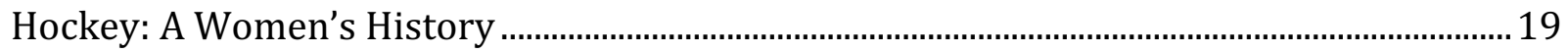

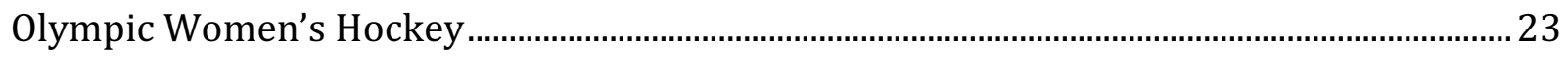

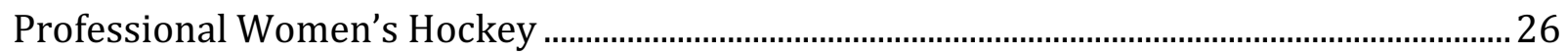

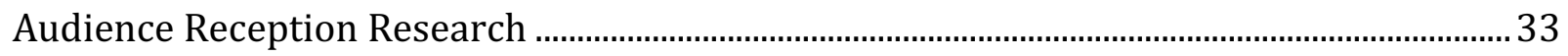

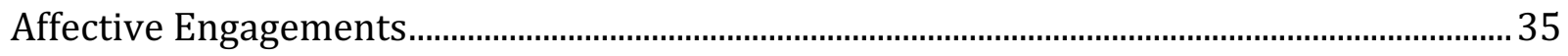

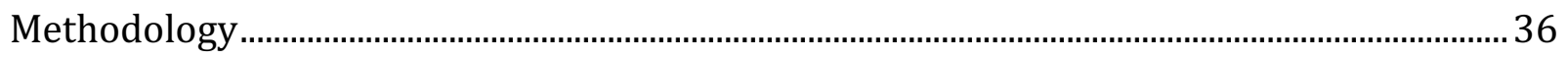

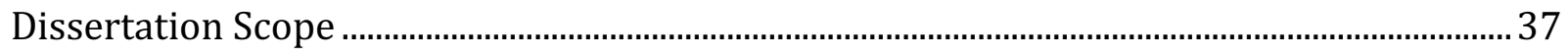

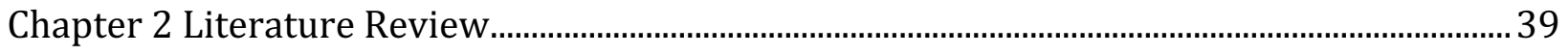




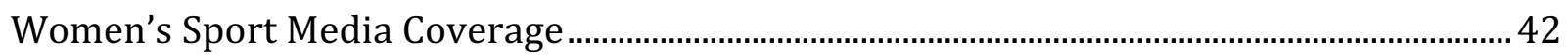

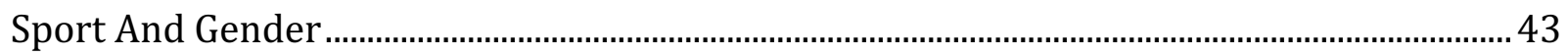

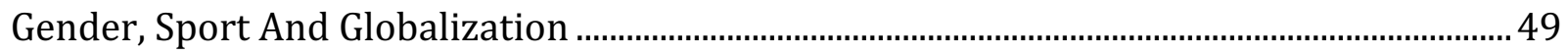

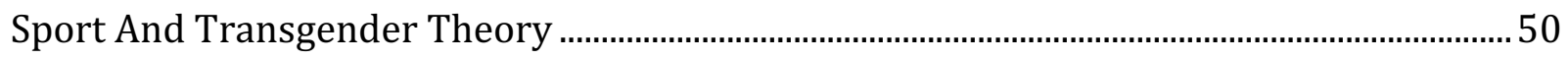

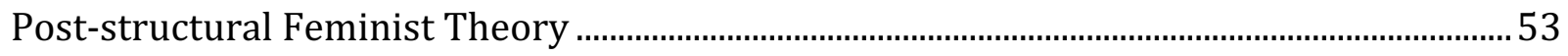

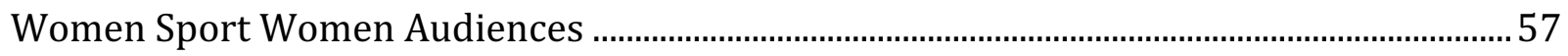

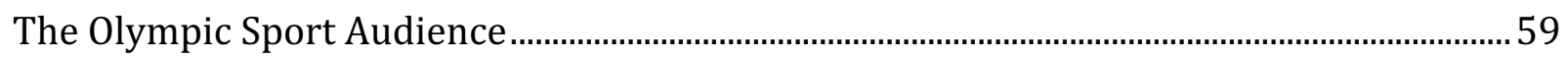

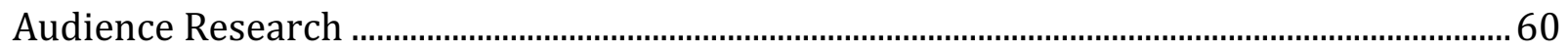

Social sciences.

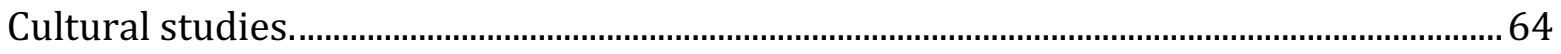

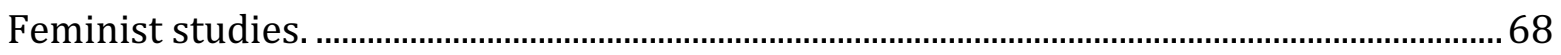

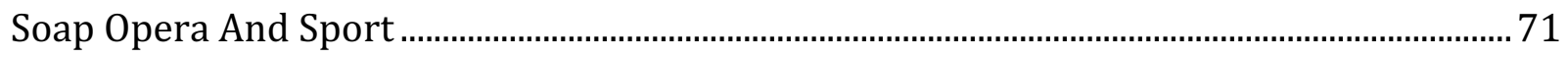

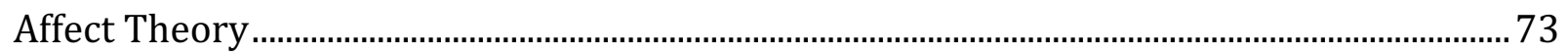

Affect And Audience Research.......................................................................................................76

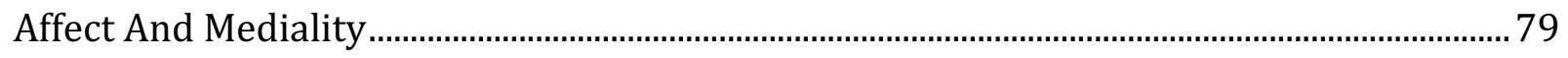

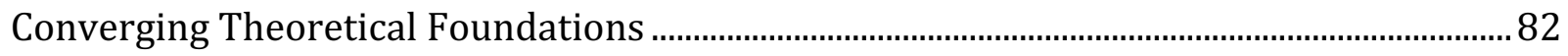

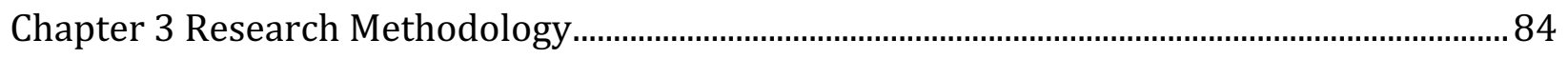

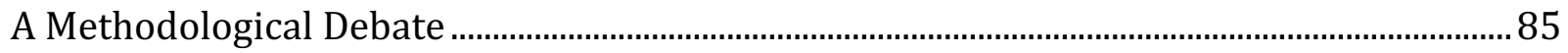

A Case For Mixed Methods Audience Research ............................................................................ 90

Sport Audience Research Methods ................................................................................................. 92 


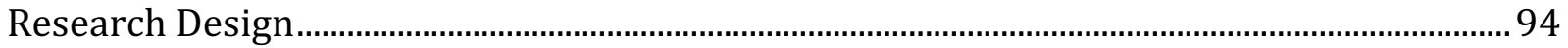

The quantitative component: the online survey....................................................................... 96

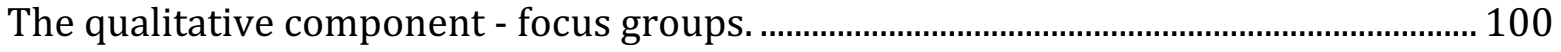

Sampling Selection..................................................................................................................... 106

Mixed Method Data Analysis ............................................................................................................. 109

Quantitative data analysis: the survey. ............................................................................... 109

Qualitative data analysis: open text survey responses........................................................ 112

Qualitative data analysis: focus groups.................................................................................. 114

Mixed Methods: A Grounded Integration .................................................................................... 118

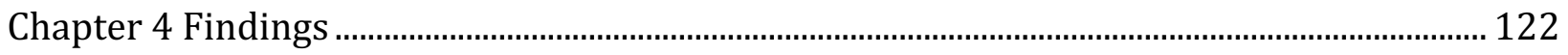

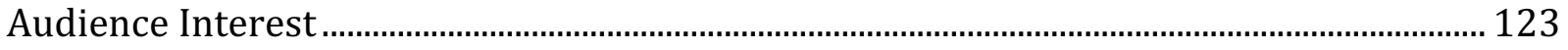

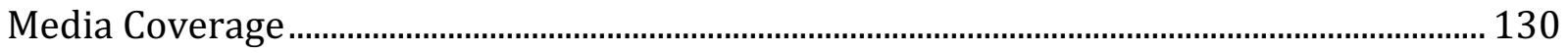

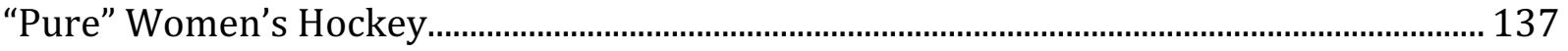

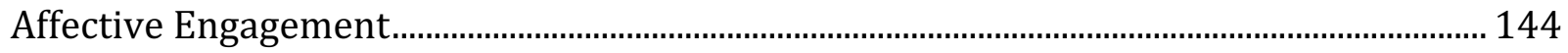

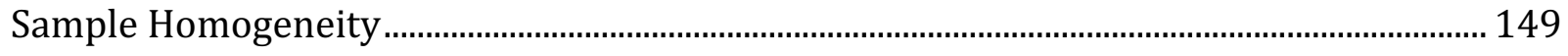

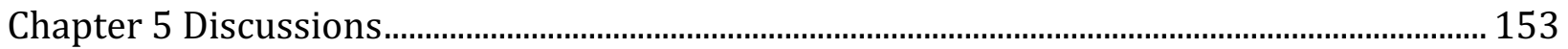

Women's Hockey Audience ....................................................................................................... 153

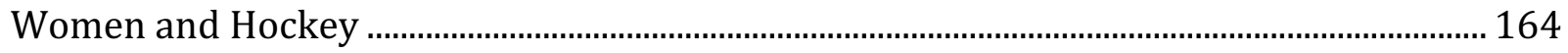

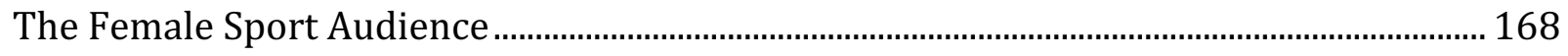

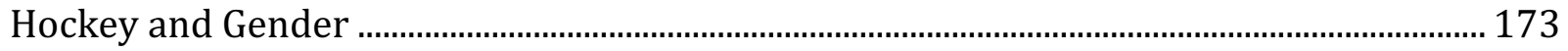


Women's "Pure" Hockey................................................................................................................ 176

The Professional Amateur ............................................................................................................... 181

Inclusive Women's Hockey ......................................................................................................... 184

Affect and Aggression ................................................................................................................ 189

Soap Opera and Sport........................................................................................................................ 206

Mixed Methods Audience Research …………………………………………………………...... 209

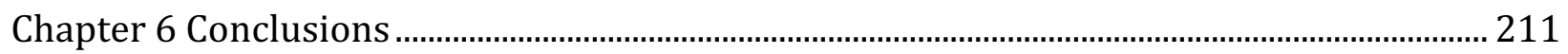

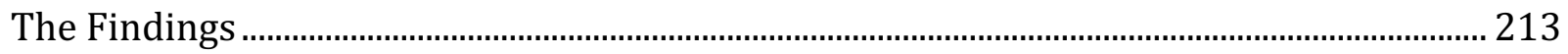

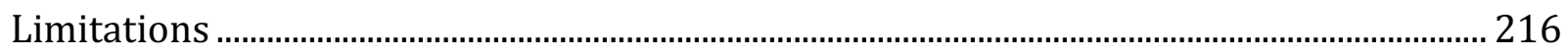

Recommendations Future Research ...................................................................................... 216

Growing The Audience For Professional Women's Hockey................................................... 219

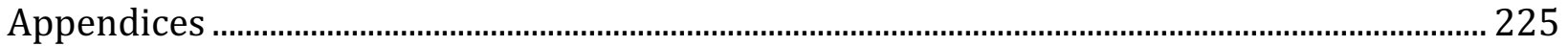

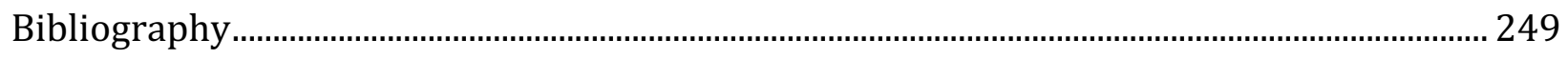




\section{List of Tables}

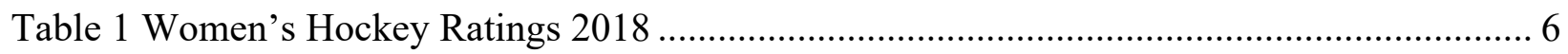

Table 2 Men's Hockey Ratings: 2018, 2014, 2010 ............................................................. 7

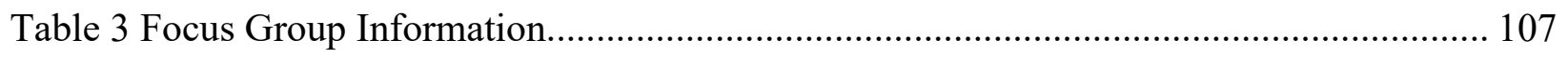

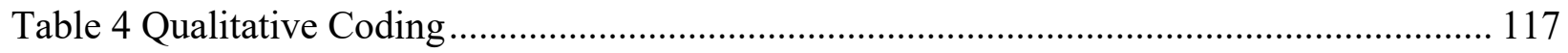

Table 5 Canadian Television (CBC) ratings for PyeongChang 2018 Olympic Winter Games.. 125

Table 6 Sample Coding: Q19) Why should women play professional hockey? ...................... 138

Table 7 Q19) Why should women play professional hockey? ............................................. 139

Table 8 Q21: Why should more people watch professional women's hockey?...................... 140

Table 9 Q21: Why should more people watch professional women's hockey?....................... 141

Table 10 Q19: Why should women play professional hockey? ......................................... 141 


\section{List of Figures}

Figure 1. Q1) Among all winter Olympic sports, how important is hockey to you?................. 123

Figure 2. In the Winter Olympics, would you rather watch Men's Hockey or Women's Hockey?

Figure 3. Q9) Are you aware that women also play professional hockey? ............................ 128

Figure 4. Q13) Are you interested in watching women play professional hockey?................. 128

Figure 5. Q11) Have you ever watched professional women's hockey?.............................. 128

Figure 6. Q12) Why haven't you watched professional women's hockey? ............................. 130

Figure 7. Q14) You would watch professional women's hockey if................................... 131

Figure 8. Q7) Do you think Olympic Women's hockey is............................................... 145

Figure 9. Q6) Watching Canadians play hockey during the Olympics makes me feel ............ 145 


\section{List of Appendices}

Appendix A - Ryerson Research Ethics Board Approval................................................. 225

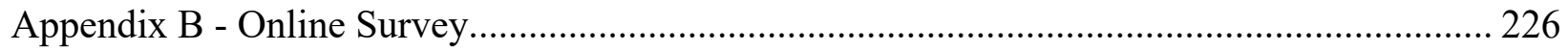

Appendix C - Online Survey Consent............................................................................ 233

Appendix D - Online Survey Recruitment Form .......................................................... 236

Appendix E - Focus Group Recruitment Form .................................................................... 238

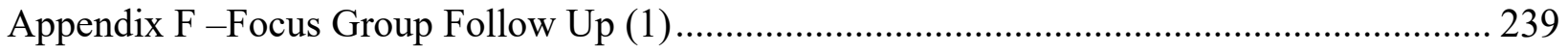

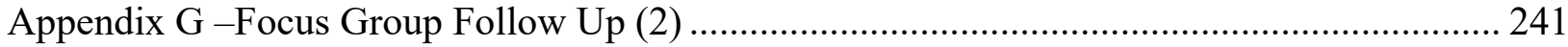

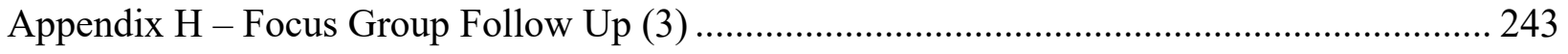

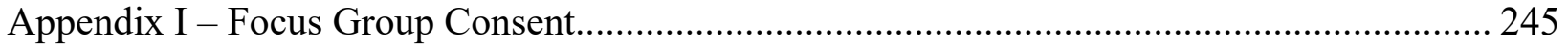




\section{Chapter 1 Introduction}

In 2015, I wrote, directed and produced a feature-length documentary called Play Fair (Gall, McIvor, \& Peeler, 2015) about women and sport in Canada and the ongoing discrimination female athletes face despite decades of activism and agitation. I identified six issues that must be addressed in order to make sport more equitable for women: participation; athlete safety; homophobia; leadership; media representation and media coverage. Issues don't make a film and so I told stories of individuals and groups that had faced these barriers; a female rugby coach who lost her job to a man when the sport was accepted by the Olympics; an aboriginal water polo player who suffered institutionalized racial discrimination and witnessed others suffer verbal and physical abuse at the hands of their male coach; a ski jumper banned from competing at the 2010 Olympics over fears of injuries to her reproductive organs; an Olympic rower who worried muscles might make her unfeminine; a lesbian hockey goalie who helped thousands of others by coming out; and a professional women's hockey team struggling for media coverage and a secure future. The film had a successful broadcast on Sportsnet as part of their PanAm Games coverage and, I am proud to say, went on to be consulted by provincial and federal bodies developing policy initiatives to support women and girls in sport in Ontario and Canada (Ontario Ministry of Tourism, Culture and Sport, 2017; Standing Committee on Canadian Heritage, 2017).

Four years later, the landscape for women and sport has changed. Tennis athlete Serena Williams is speaking out against systemic gender and racial discrimination in her sport and is receiving favourable and prominent coverage in the mainstream and sport media (Afoko, 2018; Caprino, 2018; TheUndefeated, 2018). Players from the Women’s National Basketball 
Association (WNBA) have walked away from their 2020 contracts in an effort to pressure league and team owners to address inequities in pay, resources, benefits and travel plans (D'Arcangelo, 2018; Neal, 2018; “WNBA players opt out”, 2018). Athlete safety has become front page news with the conviction of Larry Nassar, the former USA Gymnastics national team doctor sentenced to 175 years for sex crimes against athletes, and the impact statements of the more than 160 girls and women victims and their parents (Hobson, 2018). The International Olympic Committee (IOC) has publicly committed to advancing gender equality in Olympic sport and estimates 48.8\% female participation at the 2020 Tokyo Games (Armstrong, 2017). In the summer of 2015, the Fédération Internationale de Football Association (FIFA) Women's World Cup (FWWC) was watched by a record-breaking number of viewers around the world, over 750 million of them for the final match between Japan and the US, trumping all previous FWWC tournaments and even beating the men's World Cup viewing numbers, albeit in the somewhat soccer-shy United States (“FIFA Women's World Cup”, 2015).

In Canada, more Canadians watched the 2015 FWWC than the 2014 men's FIFA World Cup; other comparisons in tennis, in university level hockey and in curling, the women's events drew in significantly higher audiences than the men's (CAAWS, 2016). In 2018, the Canadian federal government committed $\$ 30$ million dollars to support "data and research and innovative practice to promote the participation of women and girls in sport, and to provide support to national sports organizations to foster the greater inclusion of women and girls in all facets of sport" (Canadian Heritage, 2019, 3rd para.). A CBC News and Sports investigation reported that over a 20-year period, 222 amateur sport coaches were charged and convicted of sex offences involving over 600 victims under the age of 18 (Ward \& Strashin, 2019). And, at the time of 
writing, Canadian women athletes are in talks with Curling Canada demanding pay equity with their male colleagues (Strong, 2019).

Despite this evidence of progression, many issues remain for women in sport. The IOC commitment to equality for female athletes must be tempered by an acknowledgement that participation percentages can mask ongoing gender-based differences including inequitable access to certain events based on distance or endurance. The number of women in leadership positions in elite sport organizations remains low (Adriaanse \& Claringbould, 2016; Adriaanse \& Schofield, 2014; Burton, 2015) and the few women who work in broadcast sport, behind and in front of the camera, continue to suffer the effects of rampant sexism and sexual harassment in the workplace (Antunovic, 2018; Everbach, 2018). The controversial practice of testing to verify a female athlete's gender, although challenged and shelved in 2015 (Branch, 2015), was reestablished in 2019 when Caster Semenya lost her appeal against an International Association of Athlete's Federation (IAAF) ruling that forced her to either stop competing, or undergo invasive hormone therapy ${ }^{1}$ (Ingle, 2019). Women's sport continues to receive a fraction of available media coverage (Billings \& Young, 2015; CAAWS, 2016; Cooky, Messner, \& Hextrum, 2013; Cooky, Messner, \& Musto, 2015; Kane, 2013), and what exists is too often characterized by a focus on female athletes' appearance rather than their athleticism (Bruce, 2016; Fink, 2015; Sherry, Osborne \& Nicholson, 2016). And, on March 31, 2019, the Canadian Women's Hockey League (CWHL) suddenly ceased operations (McGran, 2019). This leaves only one professional

\footnotetext{
${ }^{1}$ On August 19, 2009, 18-year old Caster Semenya won the gold medal in the women's 800 meters at the World Championships in Athletics in Berlin. She was told by the International Athletics Association (IAAF) to undergo a gender verification test measuring her level of androgen, a naturally occurring testosterone, to determine if she was eligible to compete in a women's event. Gender testing is a controversial practice, administered only to women, that has been discredited by scientists, activists and athletes (Dunn, 2017; Kidd, 2018; Pieper, 2016). While cleared to compete in 2010, it was revealed later that Semenya underwent hormone treatment. In April 2018, the IAAF announced new rules for gender testing. Semenya took the IAAF to court arguing the policy was discriminatory and unfair and posed health risks to female athletes (Campbell, 2019). On May 1, 2019, Semenya lost her appeal (Ingle, 2010; see Cooky \& Dworkin, 2013).
} 
women's hockey league, the National Women's Hockey League (NWHL) based in the United States. Recent reports about players unionizing and boycotting the 2019-2020 season suggest its future is also uncertain (“More trouble for women's hockey", 2019).

In my documentary, I dedicate significant screen time to women's hockey, interviewing CWHL commissioner Brenda Andress, Montreal Stars goalie and Olympian Charline LeBonté, Manitoba Bison forward Michelle Pawluk and Olympian Hayley Wickenheiser. I begin the film in the midst of a women's hockey game, ponytails flying as players chase each other down the ice. The visual juxtaposition of women playing a sport so thoroughly drenched in masculinity (Theberge, 2000, 2003; Gee, 2009) was, I argued, an effective assault on the gender normativity of sport and set the tone for the discourse of gender that I wanted the film to engage more generally. The story of women's hockey includes the struggle for a socially legitimate and economically viable professional league. My interviews with Brenda Andress and Hayley Wickenheiser about the future of professional women's hockey lodged in my brain and went on to form the basis of my doctoral dissertation research question. When asked for her thoughts about the struggle for media coverage and the low broadcast ratings for professional women's hockey games, Brenda Andress answers:

You know, every four years, the Olympics comes around, and I think last year 13.1 million individuals watched that final game, and I don't think there was one out of the 13.1 million that didn't say it was an absolutely outstanding game of hockey. And so, for us it's to say why don't you watch us after it's over? Do you not know we're alive? Do you now know where we play? And so that's a huge challenge for us. 
The same question prompted Hayley Wickenheiser to answer, with palpable frustration, that the economic model for professional women's hockey exists but needs investors and audiences to commit:

Really it's about television ratings and bums in seats and interest in the game. Every time I talk to someone, [they claim] it will happen in the next few years and it doesn't and now I am feeling impatient. Because it needs to happen and I think we're right on the cusp of it happening. Whether it will be in my playing days or not, I don't know.

It is from this place of frustration that I found myself also asking, why do millions of people watch women play hockey during the Olympics but don't watch women play in the professional leagues? Why does professional women's hockey struggle for broadcast ratings? I argue that the diminishing audiences for women's hockey is so dramatically specific as to be what Rowe (2004) refers to as a "point[s] of dissonance and resistance, the place[s] where different meanings and practices of and for sport are made and played out" (p. 8). My research focuses on the audience and the viewing inconsistency for women's hockey as one such "point of dissonance and resistance" requiring interrogation.

\section{Ratings and sport}

In sport, it always comes down to numbers. One hundredth of a second faster, a centimetre higher, a tenth of a point lower determines the difference between victory and failure. Numbers also provide the foundation for my research question: I ask why the number of Canadians who watch broadcast Olympic women's hockey is so much greater than the number of Canadians who watch broadcasts of the professional Canadian Women's Hockey League 
(CWHL). Audience ratings are often conflated and presented in bite-sized, easily digestible figures. In reality, measuring national audience ratings is complex and multi-layered. Its importance to the underlying premise of my research question means the use of ratings, specifically as ascribing social value, is unavoidable. Table 1 below shows a comparison of the (2+) average minute audience (AMA) ratings for the 2018 Olympic women's hockey gold medal game and the 2018 CWHL Clarkson Cup final:

Table 1

Women's Hockey Ratings 2018

Olympic Women's Hockey Gold Medal Game $\quad$ CWHL Clarkson Cup Final

4.8 million $^{2}$ $121,000^{3}$

Table 1

I argue that the dramatic drop of audiences from 4.8 million to 121,000 deserves examination.

Instinctually, the answer to the discrepancy is simple: winter sports benefit from the increased profile, marketing and publicity of the Olympics ( Kim, Cheong, \& Kim, 2016). Why then would women's hockey be different? The important comparison to note here is the audience ratings for men's hockey between the Olympics and the professional National Hockey League. Table 2 below notes the $(2+)$ average minute audience (AMA) ratings for the men's hockey gold medal game over the last 3 Olympics in comparison with AMA ratings for the professional men's league, the National Hockey League (NHL) Stanley Cup finals in the same year:

\footnotetext{
${ }^{2}$ Source: Numeris TV Meter, Feb. 21, 2018, CBC Total + TSN Total +SN + SN1, A2+, Wed-Thu 11p-2:30a, Total Canada, AMA, generated by InfoSys $+T V$

${ }^{3}$ Source: Sportsnet representative Ben McConnachie- Howarth, personal communication, May 21, 2018.
} 
Table 2

Men's Hockey Ratings: 2018, 2014, 2010

$\begin{array}{lcc}\underline{\text { Year }} & \underline{\text { Olympics }} & \text { Stanley Cup } \\ 2018 & 1.8 \text { million }^{4} & 2.7 \text { million }^{5} \\ 2014 & 5.7 \text { million }^{6} & 5.1 \text { million }^{7} \\ 2010 & 16.6 \text { million }^{8} & 3.3 \text { million }^{9}\end{array}$

Table 2

As Table 2 shows, the disparity between men's Olympic hockey and men's non-Olympic (NHL) hockey is evident but not consistent and nowhere near as drastic as the difference in ratings for women's hockey. I argue the relative lack of disparity between audiences for men's Olympic Hockey and professional men's hockey in the National Hockey League (NHL), coupled with the reified place the sport of hockey has in Canadian cultural discourse (Gruneau \& Whitson, 1993, 2006; Harrison \& Dopp, 2009; Horning, 2018), makes the markedly lower audience numbers for professional women's hockey a rich object of study.

These ratings measure and solidify the arguments of feminist sport activists and academics (Bernstein, 2002; Alvarez, 2018) who argue that reduced visibility from lack of media coverage translates as lack of legitimacy. Academic articles such as "Women Play Sport, But

\footnotetext{
${ }^{4}$ Numeris TV Meter, Feb. 24, 2018, CBC Total + TSN + A2+, Sat 11p-2a. Total Canada, AMA, generated by InfoSys $+T V$

${ }^{5}$ Numberis TV Meter, Jun. 7, 2018, CBC Total+, TSN+, A2+, Sat 8p-11p, Total Canada, AMA, generated by InfoSys $+T V$

${ }^{6}$ Maloney (2014)

${ }^{7}$ Numeris representative: Tom Jencks, personal correspondence, July 8, 2019.

${ }^{8}$ Zelkovich (2010)

${ }^{9}$ Numeris representative: Tom Jencks, personal correspondence, July 8, 2019.
} 
Not On TV: A Longitudinal Study of Televised Media” (Cooky et al., 2013) and "“It's Dude Time!': A Quarter Century of Excluding Women's Sports in Televised News and Highlight Shows" (Cooky et al., 2015) utilize quantitative research methods to bring into stark relief the inequality of media coverage dedicated to women's sport, results that often end up in outraged headlines touting minuscule percentages of conventional broadcasting minutes or column inches dedicated to women's sport (Alvarez, 2018; Ramirez, 2018). Even the United Nations Educational, Scientific and Cultural Organization has called for more equitable media coverage for women's sport (“UNESCO calls for fairer”, 2018). These headlines and releases in themselves become, somewhat ironically, much of the media coverage that women's sport receives. I will argue in the following chapters that media coverage that focuses primarily on the problems with women's hockey runs the risk of increasing rather than counteracting marginalization.

\section{Women's Hockey in Canada}

Perhaps as befits a bilingual country, Canada has two officially recognized national sports: ice hockey and lacrosse (Legislative Services Branch, 1994). Of these, ice hockey permeates our national consciousness, (Allain, 2008, 2014; Kidd, 2013; Gruneau \& Whitson, 2012; Vincent \& Crossman, 2015) dominating our sporting airwaves, cereal boxes, stories, songs and sweaters (Blake, 2010). This research acknowledges hockey as a pervasive social discourse in Canada that has the power to construct gendered subjectivities, especially associated with nationalism. Adams (2006) critiques hockey as an "obfuscating construction of the so-called 'ordinary Canadian', a creature whose evocation in popular political commentary helps to 
homogenize discourses about an increasingly heterogeneous population” (p. 71). The social discourse of hockey as perpetuated in the sport and non-sport media purports to represent Canadian identity but instead privileges the experiences of white men. This establishes a cultural baseline against which all other identities and experiences, differences of gender and race, are measured and found wanting, and ultimately, excluded.

Cloaked in an armour of steel and plastic, be it the toothless goon, gorgeous celebrity or just an example of Don Cherry's "good old Kingston boy" (Barber, 2013), the hockey player represents the "hyper-masculine ideals of a mythical warrior culture" (Gee, 2009, p. 585). Like so many other sports where gendered superlatives are used only when isolating the female leagues (Meân, 2010), hockey is automatically men's hockey whereas women's hockey is never just hockey. It is both cultural myth and commercial product constructed by a patriarchal society invested in narratives of gender difference, celebrating physical strength, aggression and toughness as "naturally" male and held in stark contrast against the frailty of the female constitution; and by the sport-media complex (Jhally, 1984; Messner, Dunbar, and Hunt, 2000) invested in the economic payout of hockey's corporate institutions (Gruneau \& Whitson, 2006; Phillips, 2018). The economic operation of broadcast media depends on the commodification of the audience as a product, assembled by media institutions (and in Canada with government assistance) and sold, specifically audience time, attention and purchasing power, to advertisers. The reification of hockey as naturalized Canadian identify is then an issue of power that determines who is represented and what institutions, values and bodies retain cultural and economic dominance.

In Canada, hockey lives beyond the television frame: infiltrating families through participation in or support of community and recreational leagues (Gruneau \& Whitson, 1993, 
2012; Harvey, 2006; Horning, 2018); invoked in popular discourses associated with music and film (Earle, 2002; Stevens \& Holman, 2013); and commemorated as national tragedy, as evidenced by the personal and collective demonstration of grief $^{10}$ following the deaths of the Humboldt Bronco hockey players in a Saskatchewan highway crash in April 6, 2018 (Elliot, 2018; Purdy, 2018). Implied in this tragic story, but explicit in advertising, game coverage and even in the mainstream (non-sport) media, is the message that hockey is a game that makes us Canadian, joins us together, makes us one, to cheer and to grieve as one, even though that "one" is "masculine and white" (Adams, 2006, p. 71).

Feminist scholars in sport media, communication and kinesiology have explored women's hockey as historical artefact (Adams, 2008; Hall, 2002), as a site of gender production (Theberge, 1997) and heteronormative disturbance (Pelak, 2002). Others have interrogated issues around participation (Eaton, 2012; Theberge, 1995), physiology and performance (Gilenstam, 2009), media representation (Poniatowski \& Hardin, 2012) and governance (Adams \& Stevens, 2007). Koivula (1999) examines the dearth of media coverage for women's hockey arguing that it "stands as further evidence for the ignoring of women athletes engaged in team contact sports categorized as masculine" (p. 601). Women have long been made absent from the game of hockey, both physically (off the ice) and conceptually (out of the public consciousness) (Gruneau \& Whitson, 1993; Hall, 2002; Stevens, 2006). Scholars argue that without media coverage, women and girls are excluded from sport, denied the legitimacy to compete, to be physical, to be aggressive and to embrace activities that defy constructed representations of femininity (Fink, 2015; McDonagh \& Pappano, 2008).

\footnotetext{
10 \#putyoursticksout was a social media driven campaign that saw thousands of people across the country lay symbolic hockey sticks against doors, on porches and in driveways in a show of sympathy and solidarity with the Humboldt team families (Frisk, 2018).
} 
The argument over the lack of media coverage for women's sport is classic chicken-andegg. Private broadcasters and producers argue that the audience for women's sport is too small, the cost of production too expensive, the value of airtime minutes too high to take the financial risk without guaranteeing viewers for advertisers (Gall, McIvor, \& Peeler, 2015), an audience that is presumed to be predominantly, if not exclusively, male (Cooky et al., 2013) and equally presumed to be uninterested in watching women's sport (Creedon, 1998). Activists and athletes argue an audience must be built through broadcaster investment, advocating a "if you show it they will watch" commitment, to create "a sense of interest on the part of the audience in a sport to suit the needs of the marketplace" (Creedon, 1998, p. 98). Messner, Duncan and Wachs (1996) argue that audiences for sport are constructed by sports/media complex investment, not only in airtime hours covering games and matches, but also by the supporting mechanisms that frame certain (male) events as "dramatic, historic. That they simply 'must' watch, while fans are likely to see the women's [sport] as a non-event or, at best, just another game" (p. 435).

The audience for Canadian professional women's hockey has grown, despite the fact that media coverage, in terms of games broadcast, has not increased. Since 2015, Sportsnet has broadcast three CWHL games and the Clarkson Cup final. Audience numbers for the Clarkson Cup have grown from 40,000 in 2015 (Larkin, 2015) to $110,000^{11}$ in 2018. The 2019 CWHL Clarkson Cup final broke records with 175,000 fans tuning in to watch ("Players 'shocked' as Canadian Women's", 2019). The game was broadcast nationally in Canada on Sportsnet and, for the first time, to the U.S. market on the NHL Network. Whether attributable to an increased public interest resulting from the national women's hockey team performance at the 2018 Olympic games (see below), or more substantial coverage from sports journalists, the ratings

\footnotetext{
${ }^{11}$ Ratings from Sportsnet representative Ben McConnachie-Howarth, personal communication, May 21, 2018.
} 
indicate an upward trend. Still, these ratings are far from the NHL's average audience, 2.7 million for the 2018 Stanley Cup (Rody-Mantha, 2018), nor ultimately enough to save the CWHL.

As I argue below, audience ratings are far from simple representations of actual numbers of viewers (Napoli, 2008), yet they remain a key indicator used by broadcasters and advertisers to ascribe a value to an audience and the program that can or can't draw them in. While it is unknown if the 2019 Clarkson Cup ratings played a part in the decision to fold the CWHL, the statement from the league provides some insight declaring "the business model that has been the foundation of the League is not sustainable financially" ("The Canadian Women's Hockey League", 2019, 2nd para). Sport media writers and broadcasters reported players were “devastated" (Rosen, 2019, 1st para.) and "fearful for the future of the game" (Spencer, 2019b, 1st para) even as others were predicting a phoenix-like re-birth from the ashes, including a merger with the five-team American National Women's Hockey League (NWHL), or as a subsidiary of the men's National Hockey League (NHL) (Schram, 2019). Even Olympian Hayley Wickenheiser shared a cautious optimism in her tweet following the folding of the CWHL "one step back, two steps forward, perhaps?" (Wickenheiser, 2019). At the time of writing, no new league or merger has been announced.

In the face of this unknown future, more than 200 of the world's top hockey players collectively released a statement that they will not compete in any professional games in North America during the 2019/2020 season until there is a league in place "that will showcase the greatest product of professional women's hockey in the world" (Knight, 2019) that is financially sustainable enough to pay the players, provide health care and other benefits. Weeks later, on May 20, 2019, the players unionized as the Professional Women's Hockey Players Association 
(PWHPA). There is speculation that the boycott and the players association were formed in an effort to convince the NHL to acquire the NWHL and the remains of the CWHL to form one professional women's hockey league, the Women's National Hockey League (WNHL) (Perez, 2019). Dee Spagnuolo, the lawyer representing the PWHPA, has publicly denied this motive, explaining the unionization of the players is an effort to "create a sustainable league for professional women's hockey in North America. It's the NHL's decision (whether to back the effort) and we don't speak for them" (as cited in Perez, 2019). Other high profile players, reporters and stakeholders have pegged the NHL as the solution, able to lend infrastructure, publicity and economic underwriting to a new women's league, complete with double header broadcast games to “jumpstart women's hockey” (Ryan, 2017). One reporter recommends imposing an equity tax on the millionaire NHL players to sustain a women's league (Corcoran, 2019). NHL commissioner Gary Bettman has been outspoken in his unwillingness to become involved in either the CWHL or NWHL: "I didn't believe and don't believe that the current models are sustainable in the long-term - either one of them. Unfortunately, the prophecy was correct for the CWHL" (as cited in Sadler, 2019, 3rd para). Under Bettman, the NHL will not provide the resources required to sustain the future of professional women's hockey as long as the league(s) remain "another entity" (as cited in Sadler, 2019, 11th para).

The positioning of the men's NHL as a "Deus Ex Machina" swooping in with its economic might to save women's hockey must be critiqued for both reinforcing stereotypical gender hierarchies and ignoring issues of systemic inequity in the sport-media complex. This is best interrogated as a postfeminist discursive strategy. While postfeminism as a theoretical stance has been claimed by some intersectional scholars seeking to expand feminism to include anti-foundationalist concerns (Brooke, 2002; Gillis and Munford, 2004; Lewis, 2014), 
postfeminism as a cultural practice describes an articulation of feminism as ruptured and obsolete. Rather than anti-feminism, postfeminism indicates temporality as though the goals of gender equity have been met and feminism as a political framework is no longer necessary. Feminist expression of empowerment and independence becomes a matter of individual choice, dissected and decontextualized from structural inequities, to be taken up or discarded according to immediate needs (Dean, 2010). Postfeminism underpins the commodification of feminism that sees the realization of women's independence through consumption (Tasker \& Negra, 2007). Postfeminism also reaffirms gender difference; women's empowerment comes to be represented through her sexualization (Gill, 2007; Negra, 2009). Rather than offering economic security and cultural relevance to women's hockey (a paternalistic if not patriarchal promise), positioning the NHL as owner/father of professional women's hockey is a postfeminist discursive strategy that will instead solidify its marginalization. Defined by a naturalized sexual difference that constructs its players as feminine (slower, weaker and "othered" in a sporting context), women's hockey under the NHL risks economic dependency, whatever gains to be made from the commodification of the feminist hockey player enriching corporate owners rather than the league or players themselves.

\section{The Political Economy Of Women's Hockey}

Although not always mentioned specifically, the statements about the economic sustainability of professional women's hockey refer to the audience, both spectators attending live games but, also and more valuably, broadcast television audiences in the form of ratings. Given its foundational weight to my research question and argument, it is important to both 
define and interrogate the concept. Administrative research traditions based on positivist empirical methods, or ratings, persist as an important dataset used by the production industry and advertising to assess the reach and ascribe a value to a media text (Webster, Phalen \& Lichty, 2006). Ratings, we are assured, represent the objective size of the conventional television audience and the length of time they are exposed to a specific media text. While online program streaming provides an arguably more accurate and reliable source for audience data (Calderón, Álvarez, \& Mariño 2019), conventional sport broadcasters continue to reference administrative audience data for marketing purposes and for programming and advertising decisions. In Canada, audience measurement data comes from Numeris (formerly BBM), a not-for-profit memberowned organization that dates back to 1944 . Numeris distributes data tracking measurement devices such as the Personal People Meter (PPM) to participating households across Canada. Each individual wears a customized PPM as they go throughout the day at home, in the car, at work and out shopping. The device picks up an inaudible code that is inserted into broadcast programs to measure the amount of exposure the participant has to the signal, and presumably, to the television or radio program in which it is embedded. At night, participants place the PPM on a portable charger that uploads the days' data to Numeris servers. Numeris researchers analyze the data for member broadcasters, advertisers and licensed institutions including Ryerson University, outputting ratings reports that contain audience estimates, projections based on data from a relatively small sample group. Numeris assures its users that the small sample size "does not negate the value of audience estimates as important evidence about past behaviour and as a basis for future projections and decisions" although they do include a caveat that "numbers have to be viewed realistically" ("Data Usage Guidelines", n.d., 3rd para.). 
Numeris interprets its raw data and presents it in a number of ways. Average minute audience (AMA) indicates the average number of people that watched a TV program during any 60 -second portion, usually the minute when the number peaks. Other ratings indicate reach, the summation of all audiences who have been exposed at one time or another to a program including viewers who may have been briefly or even absent-mindedly exposed to the program and its embedded signal. Numbers in the form of audience ratings shout from headlines and websites signalling glorious victory or agonizing defeat. According to the FIFA website, 750 million worldwide viewers tuned into the 2015 FIFA Women's World Cup (FIFA.com, n.d.). 103.4 million American viewers watched the 2018 Super Bowl (Otterson, 2018) and 31 million Canadians watched the 2018 Winter Olympics ("More than $85 \%$ of Canada", 2018). These impressive numbers refer to audience reach, the total number of viewers exposed to any portion of the program. Less impressive and therefore less frequently touted in the press are numbers representing average numbers of viewers and reach. For example, while 31 million Canadians may have tuned in for the 2018 Winter Olympics, this number represents the reach of the Olympics, indicating viewers who tuned in for any moment sometime over the 17 days of broadcast. Far less dramatic and celebratory is the (2+) average minute audience (AMA) which, for the CBC English-language network over the Olympics, was 3 million ("More than $85 \%$ of Canada”, 2018). The ratings I reference in this study refer to (2+) average minute audience.

Dallas Smythe (1977) theorized that the commodity of mass communication is not in meanings, messages or information but is instead the audience. Media companies produce audiences as commodities by providing content, the audience as commodity, or, more specifically, the audience's attention and potential purchasing power is then sold to advertisers. Watching commercial television is labour that produces surplus value when viewers consume the 
products advertised in and in-between program content. Boyle and Haynes (2009), Jhally (1982), and Rowe (2004) have pointed to the political economy of the sport as "a prize commodity and 'killer application' that can attract consumers to digital media products and services" (Hutchins \& Rowe, 2009, p. 358). Jhally (1982) notes the value of the sports audience is defined by gender, specifically men and their purchasing power, particularly for high-cost consumer items. Meehan (2002) argues that the commodification of the audience valued as ratings is already prejudicially gendered to assume men as both the primary earner and in charge of household spending. Broadcasters produce a male audience for sport to create greater use-value for advertisers while at the same time ignoring the inherent contradictions of the patriarchal domestic construction of women as household shoppers and women's long history as earner in the workplace.

This makes little economic sense. In capitalism, money is supposed to be the great leveller. Arbitrary social distinctions that unfairly oppress individuals are supposed to evaporate when people enter the market for goods and services as consumers, or when they offer themselves as labour. The logic of profit should drive advertisers to demand shoppers regardless of the gender, social status, race, age, ethnicity, sexual orientation, etc., of the particular people buying the bars of soap, rolls of toilet paper, or cans of beans. Why, then, do such distinctions persist in the markets for commodity ratings and commodity audiences - in markets where companies essentially trade in people? (Meehan, 2002, p. 220).

Meehan's tone of incredulity over the illogic of a system that should work logically is reminiscent of the statements from Andress and Wickenheiser above. Meehan argues the illogic is born of prejudice resulting from "ideologies naturalizing the oppression of women and working people" (p. 202) which functions to ensure a continuing marginalization in popular 
cultural discourses. In this way, ratings are a statement of cultural currency. Higher ratings are trumpeted as evidence of value, not just in terms of quality of programming, but of the scope of shared public experience.

Ryan J. Phillips (2018) examines the political economy of Hockey Night in Canada (HNIC) to argue that the hockey audience commodity has provided great use-value for public and private broadcasters, dominating the audience market and stifling the development of other (gendered) alternatives. He indicts the Canadian Broadcasting Corporation (CBC) in particular to have "perpetuated gender inequalities due to an over-reliance on advertising revenue for funding" (2018, p. 205). He goes on to argue that the low use-value (low ratings) of the audience for women's hockey is attributable to the early 1980s NHL expansion, second wave feminism and the development of a new and highly-prized audience commodity: the 18-24 year old middle-class woman. The costs of opening teams throughout Western Canada necessitated an economic infusion from a larger audience. The expansion of women into the job market, particularly pre-parental young women, was perceived to provide them with economic autonomy and independence that increased their value to advertisers. Rather than produce that audience for women's hockey, broadcasters and advertisers instead rolled them into "the pre-existing male prime-time audience for HNIC [that] had already been reproduced for over 50 years" (Phillips, 2018, p. 214). He argues that broadcasters and advertisers were and are more interested in expanding the established audience by attracting female viewers "with more widely accessible content, while not alienating its already established male prime-time audience with more coverage of women's hockey, or else with non-hockey content" (p. 213). Women's hockey is not only marginalized, it is functionally erased from the mainstream hockey audience. Where is the 
imperative for broadcasters and advertisers to provide coverage for women's hockey when it poses the risk of diluting the foundational structure of the men's hockey audience commodity?

\section{Hockey: A Women's History}

The assumption that audiences will not watch women play sport generally, or hockey more specifically, can be challenged historically. In her book The Girl and the Game, M. Ann Hall (2002) traces the history of female hockey in Canada to pre-settlement when first nations women would play shinny, using sticks to hit a small ball across the surface of a field or ice aiming for the spot between two posts. Hockey as an organized sport was first played by men in Montreal in 1875 (Gruneau \& Whitson, 1993). By the 1890s, university women's hockey teams were competing on campus and off. By 1905, women players were forming university leagues, although one team did withdraw the following year when bodychecking was not banned. Other leagues were formed by single young women drawn to the city by industrialization to work in offices, factories and stores. Competitive games were played as exhibition or for charity in front of large crowds. Ice hockey was enthusiastically embraced by thousands of women across the country, in small towns, big cities and universities who played in recreational and community leagues. Professional women's hockey dates back to 1915. The Western Club, one of a four-club league in Montreal, was owned and operated by a local entrepreneur who paid women to play full contact hockey in front of thousands of paying spectators. This was a money-making operation built on spectacle, “viewed in the same light as men's hockey, as well as the skating races, carnivals and masquerades that attracted thousands of patrons to these rinks" (Hall, 2002, 
p. 57). Players were treated like celebrities, greeted by cheering children and honour guards and regularly played a very rough game in front of thousands of spectators.

It would not last. During the depression, support for women's hockey dwindled. Economic hardship left little time for leisurely activities and few people willing to pay to watch games. Commercial sport organizations focused on securing and maintaining a male audience to access their spending capabilities. Advertisers and the media began to represent sport as a solely masculine domain; women were relegated to sporting activities that were 'appropriately feminine'. With World War II, female sports reporters were assigned to other stories while arenas and other play facilities were turned over to the government for military training. Men returning home after war joined hockey leagues that received priority on public and community rinks; soon other times were taken with the growth of minor leagues for boys. By the 1940s "the long timeout began” (McFarlane, 1994, p. 103) for women's hockey until the 1960s, although some amateur teams continued at community level with the occasional exhibition game but no formal governance structure (Adams \& Stevens, 2007). Dwindling resources and interest caused most professional teams to shut down.

In the 1970s, the restrictive constraints limiting women's participation in sport began to receive attention from the growing second wave feminist movement. Athletes and activists articulated liberal feminist critiques that called for gender equality in sport through systemic structural change. In some provincial hockey associations, a radical feminist approach was adopted including a separatist stance demanding that women control women's sport. This resulted in some unexpected conflicts. Adams and Stevens (2007) note the irony when Ontario Women's Hockey Association (OWHA) found itself arguing against Justine Blainey, a 12-year- 
old girl denied access to play on a Metro Toronto Hockey League (MTHL) boy's team. ${ }^{12}$ Blainey's case would eventually have a successful reading in front of the Ontario Human Rights Commission and led to the dismantling of a discriminatory clause that allowed exemptions to sex equality policies for athletic associations (Hall, 2002). Struggles for access continued. In 2004, Amy and Jesse Pasternak filed a complaint with the Manitoba Human Rights Commission (MHRC) against their provincial high school athletic association for refusing to allow them to try out for the boy's senior hockey team; three years later, the MHRC ruled in their favour (Eaton, 2012).

The 1980s saw expansive growth for amateur women's hockey in Canada and the development of an international division and tournaments devised to grow the game globally. It was the watershed win for the Canadian women's national hockey team at the inaugural Women's World Hockey Championships (WWHC) in 1990 that saw women's hockey claim some space in national headlines and cultural discourses (Theberge, 2000). Reid and Mason (2015) argue that the dramatic win for the Canadian team was "instrumental in showcasing the abilities of elite female players, garnering support for the inclusion of women's ice hockey in the Olympic Games, and legitimating women's hockey as an elite sport" (p. 1679). The tournament was also covered extensively by mainstream sport broadcasters (Theberge, 2000) and ushered in an era that saw a dramatic rise in participation numbers for women playing hockey in Canada and the United States (Reid \& Mason, 2015). Yet, some scholars (Fink, 2015; Hargreaves, 1994) have problematized narratives that cite increased participation as progress and therefore a "triumphant feminist tale" (Messner, 2011, p. 152) glossing over continued structural inequities around pay, around leadership and exposure. Still, Reid and Mason point to the WWHC 1990 as

\footnotetext{
${ }^{12}$ Given the current situation with professional women's hockey possibly a subsidiary of the NHL, presumably debates around separatism will reignite.
} 
the deciding factor for the inclusion of women's hockey as an Olympic sport, although it would take another eight years to be realized. The Canadian Amateur Hockey Association (CAHA) and the International Olympic Committee (IOC) had identified the WWHC as an important testing ground to demonstrate that women's hockey could be played broadly at an elite competitive level comparable to other Olympic winter sports and the tournament where international rules and regulations would be established.

The WWHC was also the site where the controversy and confusion around allowable bodychecking in women's hockey began. To resolve the conflict between European associations that allowed bodychecking and CAHA concerns that this important tournament not be riddled with injuries, officials publicly stated that bodychecking would be allowed while privately informing referees to call the games "as though bodychecking was forbidden" (Reid \& Mason, 2015, p. 1683). I will argue that this began an exercise in institutionalized gaslighting that goes on today. I will also argue that bodychecking and physically aggressive play becomes important when considering the transmission of affect in women's hockey and sport in general (see Discussions chapter below).

While the WWHC initially faced sluggish ticket sales and little interest from mainstream or sports media journalists, the introduction of a controversial jersey for the Canadian team initiated a strategic and successful marketing campaign for the tournament (Reid \& Mason, 2017). Rather than reflecting the red and white of other national sport teams, the Canadian national women's hockey team instead wore pink and white jerseys to brand the team and tournament and catch the attention of the hockey media. While initial response to the distinctive colour was negative, Reid and Mason (2017) argue that this branding strategy changed media and public perceptions of the tournament. After the opening game, not one of the 38 press 
references to pink were given in a negative context: "instead the media began to appropriate the term pink power when describing the teams' dominant performance" [italics in original] (Reid \& Mason, 2017, p. 384). Additionally, commentators and officials noted that spectators began attending games wearing clothing or carrying items that were a similar tone of pink which in turn led to important revenue-generation through sales of licensed merchandise. While successful as a branding exercise and favoured by the players at the time as a way to distinguish the women's game from the men's this must also be considered as a postfeminist discursive strategy. The reliance on a visible representation of a gendered stereotype as gimmick serves to reinforce sexual difference and the valuation of sexual difference that sees women's hockey game notable, not for its gameplay but for the culturally and ideologically loaded colour associated with it (Krane, 2001).

\section{Olympic Women's Hockey}

While the WWHC 1990 was a defining moment for women's hockey, it did not immediately lead to its inclusion as an Olympic sport. At the time, and to this day, complaints in the media and within the IOC stated that the international competition for women's hockey was and is insufficient to merit its inclusion in the Olympics (Mohorovich, 2018; Stevens, 2018). Others recognize this discourse functions to "reinforce masculine hegemony" (Vincent \& Crossman, 2012, p. 99) by refusing to acknowledge the systemic discrimination women's hockey has faced in terms of lack of opportunity, legitimacy, funding and even access to ice time. Female hockey players have struggled with issues of negative representation, more likely to be lauded in the press for their looks than their athletic prowess (Jones, Murrell \& Jackson, 1999). 
They have also suffered commentary that celebrates women's hockey while simultaneously denigrating the players as "substandard (with the standard as masculine) ... reflect[ing] a strategy of ambivalence that undermines the credibility of the athlete and women's participation in the sport" [italics in original] (Poniatowski \& Hardin, 2012, p. 624). Even off-ice, Olympic women hockey players suffer a "gender double standard" such as the criticism aimed at the Canadian women's national hockey team in 2010 for post-game celebratory alcohol consumption (FortierBrynaert, 2011; Vincent \& Crossman, 2012). Meanwhile, the Canadian players from the celebrating men's hockey team who engaged in similar behaviour, escaped censure. This public hypocrisy helps to "create and reproduce distorted images of women who engage in masculine traditions in sport" (Edwards, Jones, \& Weaving, 2013, p. 683).

Poniatowski and Hardin (2012), describe a waning interest in Olympic women's hockey, despite the decision by US broadcaster NBC to air all the women's hockey games in 2006, and again, in 2010, albeit during non-primetime hours. In Canada, the overall ratings for Olympic women's hockey has been on a downwards trend, from 2010 when the Canadian (AMA) ratings for the women's gold medal game was 7.5 million (Patton, 2018) to 6.37 million in 2014 (Vlessing, 2014) and down again to 4.8 million in 2018 ("More than 85\% of Canada", 2018). This diminishing ratings trend can be seen across the Olympics rather than just women's hockey and may be more as a result of geography and time zone challenges than interest (Rody-Mantha, 2018). A more vital comparison may be found with the 2018 PyeongChang Games when the Canadian women played for gold and secured the second highest peak ratings trumped only by the final skate of Canadian pairs Tessa Virtue and Scott Moir (Rody-Mantha, 2018) but well ahead of the Canadian men's bronze medal game watched by 2.3 million ("CBC touts Canadian Ratings”, 2018). 
The Olympics is a mega-event, a broadcast media spectacle (Billings, 2008; Sugden \& Tomlinson, 2011; Wenner \& Billings, 2017) that effectively constructs and reconstructs national identities in alignment with dominant ideological norms (Billings, Angelini, \& Wu, 2011; Billings, Brown, \& Brown, 2013; Hall \& Hodges, 1997; Horne \& Whannel, 2016; MacNeill, 1996). Gerd von der Lippe (2002) argues that nationhood as represented is always and already gendered, be it embodied historically by kings and male rulers and, more recently, by athletes: "sporting bodies are critical articulators in the construction of the symbolic making of the nation" (p. 373). These athletic bodies are stereotypically male; the hegemonic representation of masculinity as strong, aggressive and dominant intersects easily with national values of autonomy and authority (Hargreaves, 1994; Harris \& Clayton, 2002; Lines, 2004; Tervo, 2001; Wenner, 1998). However, von der Lippe (2002) argues that elite female athletes in international competitions can also represent national identity and receive more equitable media coverage in terms of amount and quality (less sexual and more athletic), provided they are successful and engaged in a sex-appropriate sport traditionally associated with the nation. Other researchers concur by noting the increased amount of media coverage afforded to female athletes during major sporting events (Bernstein, 2002; Bruce \& Scott-Chapman, 2010; Hardin, Chance, Dodd, \& Hardin, 2002). Wensing and Bruce (2003) argue that the "old rules" of traditional media coverage that demand certain gendered sports and bodies be marginalized or erased are supplanted during Olympic coverage when athletes (female and male) are framed instead by a nationalistic fervour. Even the "new rules" allowing positive representations of female athletes, albeit with an "ambivalence" that "frame their accomplishments with descriptions and images that undermine and trivialize women's efforts and successes" (Wensing \& Bruce, 2003, p. 388), are set aside to celebrate national victories (although evidently not to celebrate the celebrations, 
as above). Eastman and Billings (1999), Elueze and Jones (1998), and Kinnick (1998) note the improved quantity and quality of women's sports during the Olympics and the resulting audience numbers that put them at near ratings parity with men's sports. Janet Fink (2015), however, counters this measured optimism arguing that the media coverage for women's sport during the 2012 Summer Games contained quantitative and qualitative differences that "negatively impacts audience perceptions of sports women's athleticism” (p. 339).

\section{Professional Women's Hockey}

Broadcasts of the Canadian national women's hockey team have garnered significant ratings during the Olympics and, arguably, produced notable personalities with cultural, social and economic value (Hall, 2002; Stevens, 2018; Theberge, 2002; Wickenheiser, 2018). The professional women's hockey league in Canada, the CWHL, has struggled for publicity and relevance, despite the fact that many of the same Canadian and American Olympic athletes play on league teams. Comparing Olympic and professional women's hockey audience ratings as apples to apples requires interrogation. As mentioned earlier, there is an argument to be made that many winter Olympic sports fail to retain significant audience ratings or, indeed, take up much space in popular discourse except once every four years. Often, media coverage and public interest centres on a particular athlete; for example, Kaillie Humphries garnered international headlines for breaking the gender barrier in bobsleigh as the first woman driver in the 4-man event (Reynolds, 2016). More headlines were to follow when she left the sport in 2019 amidst a harassment complaint against Bobsleigh Canada ("Kaillie Humphries Files Harassment", 2019). Other than these significant athlete related stories, results from national or international 
bobsleigh competitions and athlete standings are generally not covered in daily sports reporting outside of the Olympics. The argument may be made that the same should therefore be true for women's hockey. I argue that there is a difference between the lack of non-Olympic coverage of bobsleigh, for example, and women's hockey simply because bobsleigh is not Canada's national sport, nor does bobsleigh construct gendered and national identities. Also, unlike hockey, there is no gendered distinction between men's bobsleigh and women's bobsleigh in terms of economic or cultural value.

Unlike women's hockey, there is a fluidity of public perception between Olympic and professional men's hockey and its players, particularly in the 2010 and 2014 Games when NHL stars played for their home countries. Even in 2018, when the NHL banned their players from the Olympics, this was considered to be bad for Olympic hockey (Friedman, 2017) and identified as the main reason for the Canadian men's poor showing and low ratings ("Canadians less interested in Olympic", 2018). Although NHL ratings experience peaks and valleys, usually depending on the standing of Canadian teams (Wagner, 2019), the everyday, cultural relevance of professional men's hockey remains significant as a cultural discourse in Canada. How then can it exclude half the population?

Professional women's hockey is a difficult story of slower growth and continuing struggles with infrastructural and economic instability. In 1999, the National Women's Hockey League (CAN-NWHL) ${ }^{13}$ was established in Canada with four teams forming an eastern division in Quebec and five teams as the "western" division in Ontario (Hall, 2002; Theberge, 2002). The league was defined as professional although the players were not paid, nor was equipment

\footnotetext{
${ }^{13}$ This should not be confused with the current iteration of the National Women's Hockey League (NWHL) based in the United States. I have adopted an acronym for distinction: the Canadian version of the National Women's Hockey team is indicated by CAN-NWHL.
} 
covered (Hall, 2002). The league eventually expanded west to include teams from British Columbia and Alberta, although only until the 2003-04 season when travel costs became prohibitive. This prompted the western teams to drop out to form their own league, the Western Women's Hockey League (WWHL). However, by the 2006-07 season, the WWHL (now expanded to include five teams from Alberta, British Columbia, Saskatchewan and Minnesota) merged again with the CAN-NWHL to form a western division while the central division was based in Ontario and an eastern division in Quebec. To counter the costs of travel, the league planned for the western division to play amongst itself while the central and eastern division would play for a place in the divisional finals. The separation, while promising economic viability, led instead to another league breakdown and further disarray amongst leaders and officials. With no infrastructure or funding and little support from hockey organizations outside the OWHA, the 2006-07 season would be the Can-NWHL's last (Hewings, 2006).

As members of the Canadian and American Olympic teams, players from the former Can-NWHL wanted to continue to play hockey at an elite level. They came together in 2007 to form the CWHL. Like its predecessor, the CWHL formed as a professional league; although again, the players were not paid. In 2008, Brenda Andress stepped into the role of commissioner and, along with some of the star players, became the public face of the league, a position she occupied for 11 years before stepping down in July 2018. At the time of Andress' appointment, the CWHL had just lost a team, reducing the league to just six franchises (Annie, 2018). The WWHL was still operating in the western provinces in, if not direct competition with the CWHL, then certainly posing a threat of dilution of available resources. In the 2008-09 season, Hockey Canada established a national women's hockey tournament that would see the WWHL and CWHL finalist and champions face off. The 2009 tournament prize was the Clarkson Cup, 
originally commissioned by former Governor General Adrienne Clarkson for a Can-NWHL championship in 2006 and then shelved for two years following the league's dissolution and a copyright dispute between Hockey Canada, Clarkson and the artists who created the cup (“Clarkson Cup in Limbo", 2007).

In 2010, under Andress' leadership, the CWHL restructured some of their Canadian teams, expanded into the United States with two teams and ran its first draft. In 2011, the CWHL announced that the WWHL was dissolving and the teams would merge into one women's league under the CWHL. This never happened. Instead, a dispute between the leagues left two WWHL teams remaining, one in Manitoba and the other in Minnesota, while the other two merged to form the Calgary Inferno as a CWHL team ("Minnesota Whitecaps cry foul”, 2012). In 2012, the CWHL announced a partnership between the Toronto Furies and Calgary Inferno with their respective NHL teams; the Maple Leafs and the Flames would provide additional funding to cover coaches, travel and equipment costs and access to marketing teams ("Leafs announce partnership agreement", 2012; "Flames partner with CWHL", 2012). In 2014, fresh off the Canadian national team's gold medal win, the league hosted its first All Star Game drawing in more than 7,000 spectators to Toronto's Air Canada Centre (“All-Star Game", 2014). The following year, the CWHL established a third partnership with the NHL, this time re-branding the Montreal Stars as Les Canadiennes de Montreal to align more closely with the "extended Canadiens family" ("NHL Canadiens, CWHL Stars”, 2015). In 2016, the CWHL formed a partnership with the NHL's Ottawa Senators to host the Clarkson Cup final in their home stadium for the following two years ("CWHL and Ottawa Senators announce", 2016). In 2017, the CWHL expanded with two teams in China that merged into one team for the 2018/19 season. Establishing a franchise in China allowed the women's game to grow internationally and, more 
specifically, created the opportunity to build a competitive home team when China hosts the Olympics in 2022 (Andress, personal communication, June 14, 2018; Rutherford, 2018). While the Chinese teams did not pay an expansion fee, they did sign a five-year license agreement with the CWHL that created sponsorship opportunities and allowed the league to pay the players a salary for the first time with a salary cap of $\$ 100,000$ for each team. The only exception was with the players on the Chinese teams who earned a salary reported to be close to $\$ 100,000$ each, the same as the total salary cap for the five other teams in the league (Rutherford, 2018).

Some sports reporters (Berkman, 2017; Brady, 2017; Spencer, 2017) suggest that the CWHL began to pay their players in response to the pressure coming from the newly formed professional women's hockey league in the United States. The National Women's Hockey League (NWHL) was established in 2015 and was the first professional women's hockey team to hire salaried players (Peters, 2015). Originally offering salaries as high as $\$ 26,000$ annually, that number was later reduced for 2016-17 season (Gordon, 2016) putting them more closely in line with the newly established CWHL payment of $\$ 2,000$ to $\$ 10,000$ per season (Murphy, 2017). Acknowledging this didn't come close to a living wage, Andress continued to advocate for league sustainability through slow, steady growth (Andress, personal communication, June 14, 2018). The NWHL, on the other hand, grew quickly in the untapped market of post-collegiate women's hockey in north-eastern America. Press accounts from Canada paint the NWHL as upstart usurper, describing the league comparison almost as a battle between good and evil: The NWHL has a measly 16-game regular-season schedule, while the CWHL plays 28 ; the CWHL is a not-for-profit, started by players and run by a commissioner who's advised in part by a board of directors, while the NWHL is a business started by a former NCAA player and funded by private investors. (Rutherford, 2019) 
This story presents the two leagues locked in an almost existential conflict with the CWHL "notfor-profit" sensibilities prized in stark contrast to the commercial ambitions of the NWHL. Certainly, the NWHL and its commissioner Dani Rylan have battled lawsuits and scandals including accusations of deceptive business practices from investors demanding damages and repayment (Wawrow, 2016; Neale, 2016), a contested trademark of the use of its name in Canada (Spencer, 2016) and a 2016 anonymous email leak with unverified correspondences painting Rylan as dishonest, untrustworthy and crooked (Neale, 2016). I argue that the rhetoric that envisions women's hockey as a "pure", morally superior iteration of the game for resisting the lure of crass commercialization has been a commonly invoked description throughout its history. The word "pure" is loaded with specifically gendered cultural, social, historical and religious meaning. From the Victorian "true womanhood" construction of femininity that demanded women be modest, submissive, and innocent (Campbell, 1989; Roberts, 2002; Welter, 1966) to current perceptions of the inherent probity of the female politician, (Goetz, 2007), the characterization of women as "pure" re-establishes "essentialist notions of women's higher moral nature and their propensity to bring their finer moral sensibilities to bear on public life" (p. 90). It also functions to deter commercial ambitions, even while used to advocate for the goals of economic sustainability and pay equity.

Relations between the two professional women's leagues remained frosty (Friedman, 2019) even as players, reporters and the leagues' commissioners publicly pondered the possible benefits of merging to form one professional league (Brady, 2018b; Clipperton, 2019; Spencer, 2019a). When Brenda Andress stepped down as CWHL commissioner on July 31, 2018, she was replaced by interim commissioner Jayna Hefford, who would, in eight months time, announce the folding of the CWHL on March 31, 2019 ("Interim CWHL commissioner defends", 2019). 
Since then, the NWHL first announced and then cancelled plans to expand into the Toronto and Montreal markets ("NWHL cancels expansion plans", 2019) while two NHL teams have severed their connections with their NWHL counterparts (Kaplan, 2019). With this news and the planned player boycott mentioned above, the 2019-20 professional women's hockey season looks far from secure.

As Sportsnet reporter Kristina Rutherford (2019) attempts to summarize the state of professional women's hockey, even pre-CWHL closure, her frustration is clear. She describes professional women's hockey history as a "confusing mess" (6th para.), stuck in a dizzying pendulum between success and failure. She points to Kendall Coyne-Schofield's well publicized performance at the 2019 NHL All Stars, her high-speed skate representing "the potential of women's hockey when it's promoted and funded and features a best-on-best" (5th para.). Rutherford claims high profile events such as this and the resulting public attention will convince audiences to watch broadcast professional women's hockey games. The argument is that in order to become legitimate hockey players worthy of significant public attention (and audience ratings and the financial benefits that result from this), the women must be seen to be compete, if not with the men, then certainly within the male dominated environment of the NHL. The underlying assumption reflects the ideological underpinnings of gender difference: women hockey players are naturally perceived to be weaker, slower, less skilled, than men, until they can prove otherwise. Counter-arguments claim that audiences simply need to be exposed to women's hockey in order to be converted. And yet, every four years, audiences across Canada are exposed to the high quality of women's play. The issue cannot be mere exposure. What do audiences think of women's hockey? How do they decide to watch or not watch professional women play? While scholars develop theories about representation, exposure and hegemonic gender identities, 
what does the watching and even non-watching audience think and feel about professional women's hockey? To fully explore the intersection between hockey, gender and TV, I turn to audience reception research as a theoretical framework.

\section{Audience Reception Research}

Audience scholarship has a long and complicated history (Grey \& Lotz, 2012;

Livingstone, 1993), fraught with competing epistemological, ontological, methodological and paradigmatic arguments. Understanding what an audience is, what they do with media and what media does to them, has prompted academics and advertisers alike to research audiences through a variety of theoretical lenses. Early audience researchers feared the negative effects of propaganda and approached the audience as passive dupes unable to moderate media influences (Lasswell, 1927). While media effects research on the influence of television and digital texts continues, particularly to answer concerns over the distribution of violent content to young people (Anderson, 2016; Savage \& Yancey, 2008), I align with scholars who consider the audience to be active participants in finding, making and interpreting meaning from television and media texts in their everyday lives (Ang, 1985, 2007; Brunsdon, 1981, 2000; Fiske, 1987, 2001; Hall, 1980; Morley, 1986, 1993, 2006). Following Stuart Hall's (1980) canonical work in Encoding/Decoding, I acknowledge the semiotic function of media texts (traditionally broadcast television programs) that create and communicate meaning through the distribution of encoded messages (the signifier) to be decoded by the audience (signified) and in the interpretation dominant ideological norms are reinforced. Rather than the linear "sender - message-receiver" model of early media effects, audience reception research understands the encoding/decoding 
process as circular and layered; audiences find multiple meanings and make varied engagements with television texts in their everyday lives, dependent on context, accepting, rejecting and/or negotiating encoded messages in ways that are neither discrete nor predictable (Morley, 1980). I am informed by John Fiske's (1986) argument that popular television texts must provide opportunities for audiences to find more than one meaning. While polysemic television texts are embedded with dominant cultural messages there must be room for audience negotiation and resistance. Pleasure comes from interpretation; audiences must resolve the contradiction between a program's dominant "preferred" message and their diverse life experiences.

My research also considers the political economy of women's hockey and recognizes the audience as a commodity to be bought, sold and traded by commercial institutions (Jhally \& Livant, 1986; Smythe, 1977) and as consumers sought after by advertisers and broadcasters alike (Napoli, 2003; Bogart, 1996). My research seeks to understand how audiences interpret women's hockey as a television text but it also asks why audiences will not watch. Given that audiences cannot watch what is not broadcast, the question demands an examination of the issue of media coverage for women's sport and the economic, social and political foundations that lead broadcasters and sport media to support some programs and not others. I seek to synthesize multiple theoretical and methodological processes to explore the ways audiences engage with and perceive women's hockey as a media/television text and the implications this has for the construction or possible disruption of gendered normativity.

Feminist scholars, long interested in popular texts as a "natural site of identity formation and empowerment" find in audience reception research an articulation "of images and narratives valuable less as a means of representing reality than as motifs available for contesting, rewriting and recoding" (Karlyn, 2003, p. 8). Feminist audience work has focused on specifically gendered 
texts such as television soap operas (Blumenthal, 1997; Brown, 1994; Brunsdon, 2000; Hobson, 1982; Mumford, 1995) as a site of feminine resistance. Sport scholars focused on the "other soap opera" (O’Connor \& Boyle, 1993) sport media's construction of hegemonic masculinity (Adams, 2011; Allain, 2011; Connell, 2008) and sexualized femininity (Cahn, 1994; Fausto-Sterling, 2000; Kane, LaVoi \& Fink, 2013). I engage with feminist theory to consider how audiences negotiate with representations of women playing a sport that has been so thoroughly constructed as hypermasculine (Gee, 2009) and to understand its lack of media coverage as a contradiction between patriarchy and capitalism (Meehan, 2002).

\section{Affective Engagements}

As much as audiences engage with television texts in a cognitive process, some scholars (Hirdman, 2015, Bociurkiw 2011, Gibbs 2011) have theorized the role and import of affect in media and communication and specifically television texts (Kavka, 2008). Affect indicates emotion to some (Ahmed, 2004), or gut instinct to others (Gibbs, 2011) minus the cognitive machinery that links feeling to emotion. I argue that sport is a media text that communicates most efficiently through affect, or, as Hundley and Billings (2010) would have it, sport audiences "consume identity-oriented messages in a peripheral rather than central processing route" (p. 8). My research identifies these "peripheral" processing routes as affective flow. Consider the classic image of the sports viewing fan, breath held, hands clenched, brow sweating, they sit at seats' edge, poised for victory leap or dejected slump if the goal is scored, the pass blocked, the jump landed or the ball caught. Even in the most cerebrally strategic games, golf for example, the roar breaking the silence of a tension-filled putt is surely a form of what Linda Williams 
(1989) describes as the "money-shot ... visual evidence of the mechanical 'truth' of bodily pleasure caught in involuntary spasm: the ultimate and uncontrollable - ultimate because uncontrollable — confession of sexual pleasure in the climax of orgasm" (p. 101). Lindsey J. Meân (2001) argues the emotional connection between sport and identity "accounts for its significance as a globalized discourse that implicitly and explicitly constructs wider social practices such as gender" (Meân, 2009, p. 67). In Canada, the discourse of hockey constructs national identity with an implicit gender bias that, according to Dopp and Harrison, has avoided critical analysis because of its "possessive emotionality" (2009, p. 9). I argue that hockey's "possessive emotionality" is equally gendered. Playing and watching hockey as an affective activity is associated specifically with the male body and denied to women.

\section{Methodology}

While Joke Hermes (2014) described the qualitative methods of much audience reception as being the "best possible expression of feminist engagement in media studies" (p. 61), audience studies have traditionally engaged either quantitative or qualitative methods depending on disciplines and epistemologies. Administrative audience researchers from the social sciences provide empirical research for business, academic and government agencies utilizing quantitative methods to gather statistical data on audience behaviour while scholars from cultural studies and the humanities have relied on qualitative methods to understand how and why audiences engage with various media texts. In response to this debate and calls for methodological convergence (Livingstone, 2013; Morley, 2006, Schrøder, 2013, 2019), I utilized a mixed method research design integrating an online survey (quantitative) and focus groups (qualitative) with audiences of Olympic women's hockey. While data was collected separately in the linear fashion of an 
explanatory sequential design (Creswell \& Plano Clark, 2011) that demands quantitative data be collected first and then further explored in the qualitative research component, data analysis and interpretation allows a more circular integration of findings. This way, data is collected that is both broad and rich, that draws from a large sample size with the survey but allows an in-depth ethnographic investigation of audience's interpretations, thoughts, viewing habits and feelings in focus groups (Clough \& Nutbrown, 2012).

\section{Dissertation Scope}

In Chapter 2, I establish my research as a convergence between varied scholarly traditions including sport media and gender, audience reception studies, affect theory and feminist media studies. My review of literature aims to define a broad theoretical framework that will allow me to consider the women's hockey audience as a cultural and economic product and an example of patriarchal and capitalist contradictions. In Chapter 3, I explore the varied and competing methodological traditions utilized in audience studies from quantitative to qualitative and argue for the suitability of mixed methods for this study. I include the reasoning and details for the study's design and implementation as well as descriptions of the results and processes of analyses. In Chapter 4, I present the findings of the study, mixing the quantitative and qualitative results in a constant comparison to establish ways respondents and participants engage with, frame and watch or not watch professional women's hockey. In Chapter 5, I present a discussion of the findings within the context of the aims of my research study and within the existing literature, looking specifically at if and how audiences affectively engage with women's hockey as a media text and cultural product. In Chapter 6, I provide conclusions and describe how this 
research contributes to the field of audience studies, sport media and gender scholarship as well as affect theory. I detail its challenges and limitations and make recommendations for future academic research. 


\section{Chapter 2 Literature Review}

Of the many tearful tributes to the late Canadian musician and Tragically Hip frontman Gord Downie, the video created and broadcast by Hockey Night in Canada ("Hockey Night in Canada", 2016) demonstrates most effectively the seamless interweaving of narratives between Downie as rock star, Downie as hockey player/fan and ultimately, Downie as Canadian. The tribute combines a soundtrack of Downie's voice and music, with a montage of images that cuts between concert shots, famous NHL winning goals, Downie posing with players and fans, and shots of famed hockey heroes such as Sidney Crosby, Wayne Gretzky and even Coach's Corner's Don Cherry. The implication is neither subtle nor uncommon: Downie, the voice of one of Canada's most iconic bands, singing musical references to northern landscapes and social justice, along with his well-known love of hockey and goalies in particular, embodies an imagined Canadian identity where masculinity, nationalism and hockey intersect, become codified and then verified by their association. This reification of nationalism through hockey and masculinity is a multi-directional verification process; each image assures the other of its heroic status.

Given its ideological weight in Canada, it is no surprise that hockey has been the focus of much serious scholarly analysis. It is, however, a relatively new field of study for critical sports scholars who want to take "the game seriously as a place where academics might find real, meaningful fodder for the study of society and culture, and the expression of power in a variety of forms, physical and psychological” (Stevens \& Holman, 2013, p. 251). Gruneau and Whitson's (1993) foundational book Hockey Night in Canada is considered by many (Blake, 2010; Jamieson, 2014; Stevens \& Holman, 2013) to be one of the first to engage in a critical analysis of the game as a social discourse and cultural product. The book inspired the launch of 
"hockey studies" as a legitimate multidisciplinary subfield of sport studies. Men's hockey has been interrogated and problematized as a conflation of Canadian identity, masculinity and nationalism (Adams, 2006, 2011; Atkinson, 2010; Allain, 2011; Gee, 2009; Gruneau \& Whitson, 1993; Langley, 2003; Robidoux, 2001, 2002; Watson, 2017) that creates discourses of patriarchy represented in the media and reinforced in community locker rooms. Less attention has been paid to women's hockey. Theberge $(1997,2000)$ created an extensive body of work on women's hockey, particularly her embedded ethnographic research with the Blades, a team of elite-women players in the Provincial Women's Hockey League. She explores women's hockey as a series of complex practices and constant contradictions where the physicality of play both challenges and defines gender identities, promises joy while threatening censure and, ultimately, creates community. Other scholars recount the history of women's hockey (Hall, 2002; Norton, 2009; Etue \& Williams, 1996); critique the institutional limitations in women's hockey governance (Adams \& Stevens, 2007); interrogate transnational women's hockey as a problem of parity (Stevens, 2018); and question the progressive narratives of growing participation rates in girls hockey (Adams \& Leavitt, 2018). Few have critically examined women's hockey as a media text or interrogated issues of representation and coverage (Poniatowski and Hardin, 2012; Sparks and Westgate, 2002). Vincent and Crossman (2012) conducted a comparative content analysis of media coverage of men's and women's hockey teams at the 2010 Winter Olympics. Their findings indicate mainstream media create narratives that reaffirm dominant gender ideologies, observing that the women players are more likely to be described in terms that "failed to fully recognize the athleticism and agency of the women's teams" (Vincent \& Crossman, 2012, p. 104). 
Considering the state of the subfield of 'hockey studies', Stevens and Holman (2013) acknowledge the body of "rich and growing and intellectually profound" literature that forms the basis of an academic canon. Celebrating these achievements, they also include a cautionary tale of sorts about the need to consider hockey from below the ivory tower of academia looking "closer to the game ... making connections with those who experience the sport daily or weekly at rinkside" (Stevens \& Holman, 2013, p. 252). Stacy L. Lorenz (2015) calls for research that does more than interpret hockey texts; " it is important to acknowledge that media sources "can reveal the ideas and images in widest currency, but they cannot finally tell us how their audiences understood them" (p. 109). MacNeill (1996) engages production studies to consider how broadcasters and the NHL encode messages in the media text but there has been shockingly little scholarship examining how these messages are decoded. Research into the hockey audience has not been well served and even less is known about the audiences for women's hockey.

It is indisputable that the audience for professional men's hockey in Canada has been and continues to be significantly larger than the audience for professional women's hockey. With the exception of Olympics where audience numbers approach parity, the disparity between the millions who watch the NHL and the tens of the thousands who tune into women's hockey is clear. My research question asks how does Olympic women's hockey entice viewers to tune in and why do those viewers so decidedly tune out after the Games have ended? In this chapter, I will review the existing literature exploring hockey as a patriarchal discourse and women's hockey as both a challenge to and victim of hegemonic masculinity. The theoretical foundation of this work is at the intersection of cultural studies, sport media scholarship and affect theory; I'll review the literature of each theory as related to and informed by feminist media theory. 


\section{Women's Sport Media Coverage}

Scholarly research on women's sport and the audience has primarily consisted of quantitative research measuring websites, minutes or column inches of media coverage. Often repeated in outraged headlines, the numbers are shocking: researchers report that while $40 \%$ of all athletes are female, women's sport receives a mere 4\% of media coverage (CAAWS, 2016; Cooky et al., 2013) and that women's sports receive less airtime than they did 10 years ago (Cooky et al., 2015). Even amidst the increased publicity of the 2012 Olympics, researchers report that women's sport received an average of only $4.5 \%$ of British press coverage; a measly figure that decreased to 2.9\% after the Olympics concluded (Packer et al., 2014). The lack of media coverage for women's sport has a number of implications for the audience and for female athletes. Hardin and Greer (2009) argue that despite greater gender inclusivity in personal sport (in individual fitness and on community teams), unless portrayed on a broader level through mass media, traditional stereotypes remain unchallenged, especially when it comes to defining traditional gender appropriate sport. Researchers have established a direct association between greater media coverage and increased participation (CAAWS, 2016). Other researchers relate lack of media coverage to the trend of girls who drop out of sport in large numbers at adolescence (Bailey, Wellard, \& Dismore, 2005; Johnstone \& Millar, 2012). Duncan and Hasbrook (2002) turn to media studies to argue that television coverage of team sports and some individual sports provides insight into the exercise of power in society: "sport tells us, through its lopsided distribution of women into 'female-appropriate' individual sports, men into team sports and certain 'manly' individual sports, how this power is withheld from women and how it is accorded to men" (p. 86). Without representation, women's sports reaffirm the gender binary 
most powerfully through their absence. The female body, different and invisible, serves to make sport more fully male.

\section{Sport And Gender}

Gender differences are the bricks and mortar of sporting culture and structure; a built in binary based on biology. Pfister (2016) argues that sport does more than perform sexual difference, it does so within a specifically defined gender hierarchy that defines certain sports to be acceptable for certain genders as though through naturalized individual preferences that reflect "social norms, values and expectations" (p. 40). Emanating from and enacted on the body, in sport, gender becomes entwined with an essentialism that anchors women as naturally weaker, less physically competent and of less value. This can be seen most clearly through gender testing. At the 2014 Commonwealth Games in Glasgow, Dutee Chand, a runner from India, failed a hyperandrogen test and was barred from competing (Macur, 2017). Whether high levels of natural testosterone give female competitors an unfair advantage is, in many ways, beside the point. These tests are only issued to female athletes, particularly those who do not fit into traditional Western expectations of femininity and are more likely to have "their biological standing as female athletes called into question" (Kane, 1995, p. 210). Nor are other "unfair advantages such as height, centre of gravity or, other genetic differences in male athletes, grounds for exclusion:

[Michael] Phelps possesses a proportionately vast wingspan, for example. Double-jointed ankles give his kick unusual range. In a quirk that borders on supernatural, Phelps apparently produces just half the lactic acid of a typical athlete — and since lactic acid 
causes fatigue, he's simply better equipped at a biological level to excel in his sport... Nobody suggested he should be forced to have corrective surgery on his double-jointed ankles, nobody decided he should take medication to boost his lactic levels. (Hesse, 2019, 1st para.)

Gender testing must be seen through an intersectional lens that considers issues of race and class, as well as gender. That these women hail mostly from the Global South, often without the economic benefits of corporate sponsorship, indicates clearly that sport as an institution and industry, buttressed and supported by government and media, reaffirms and reproduces sex-, gender-, race and class- based forms of social injustice (Cavanaugh \& Sykes, 2006; Cole, 2000; Travers, 2008).

Feminist sport scholars such as Adams (2006), Birrell and Cole (1994), Cavanaugh and Sykes (2006), Cole (2000), Kane (1995), Sykes (2006) and Travers (2008) have sought to highlight the ways in which sport reaffirms the sex/gender binary as inherent, natural and inevitable. Some scholars (Messner, 1988; Koivula, 1999; Kane, 1996) critically analyze media representations of female athletes and call out homophobia in sport (Wright \& Clarke, 1999; Adams, 2011). Chalabaev, Sarrazin, Fontayne, Boiché, and Clément-Guillotin (2013) suggest that the representation of gender stereotypes in sport have specific implications for how females perceive themselves as athletes and consider their legitimacy to compete, to be physical, to be aggressive, and to embrace activities that defy constructed representations of femininity. Much important academic and advocacy work exposes systemic discrimination and inequities in an effort to make sport a better place for women. For example, Sherry, Osborne, and Nicholson (2016) identify a set of categories that define the limited representation of female athletes in print images. Photos tend to show female athletes in gender appropriate sports such as skating, 
swimming or tennis and often in passive positions rather than active. Females are more likely to be shown playing individual sports as though the representation of women in team sports risks a suggestion of lesbianism. They also note the persistent sexualization and objectification of female athletes as a subtle but effective strategy to minimize athletic performance and reassert traditional notions of femininity (see also, Bem, 1981; McVee, Dunsmore, \& Gavelek, 2005).

The prioritization of beauty over physical prowess is so intrinsic to the structures and ideology of sport that even female athletes themselves succumb to the message. Kane, LaVoi, and Fink (2013) gathered a focus group of elite female athletes to show them images of women in sport some celebrating on-court competency and others showing women off-field emulating the style of soft-core porn. Participants were asked to choose the image they believed would most effectively generate public interest. While a little more than half indicated their preference for the photos celebrating athletic competency, $47 \%$ of participants chose the soft-core porn images to be the best for self-representation. This "dual-identity" between the competent athlete on the field who prefers prescribed definitions of femininity off the field was most commonly articulated by those participants who played on teams: "for these athletes, performances of femininity were considered an effective counterweight to what they believed were stereotypes linking women's sports to a lesbian presence" (Kane, et al., 2013, p. 294).

While no less dedicated to social change, other feminist scholars interrogate and challenge the gender binary, illuminating how culture shapes and creates the body (Balsamo, 1995; Butler, 1993; Dreger, 1998, Dworkin \& Wachs, 2009; Fausto-Sterling, 2000). In this approach, the body is not fixed genetic material shaped by natural evolution but instead is the product of historically specific practices shaped through relations of power. As Cooky et al. (2013) put it: "specific social roles imbue bodies with particular capacities and physicality, there 
may be less in common biologically between bodies of female domestic workers and bodies of female athletes, despite the specific sex categorization of those bodies" (p. 32). Gender construction regulates not only behaviour or performance, but also a physical structure that is capable of change. Fausto-Sterling (2000) insists we move away from genetic or biological classifications to offer new conceptualizations of sex categories stating: "a body's sex is too complex. There is no either/or. Rather there are shades of difference" (Fausto-Sterling, 2000, p. 3). For these scholars, as well as some athletes and advocates, the goal isn't simply making sport equitable for women; it is disavowing gender difference entirely to be replaced instead by sexintegration, a physical meritocracy, the inevitable end to closing the "muscle gap" (Messner, 1988). As indicated in the title of the book, separate sport does not mean equal sport (McDonagh \& Pappano, 2007), this is a controversial issue with other feminist scholars arguing the need for women to maintain separate sporting spaces to minimize the risk of violence and physical injury, and to ensure the hard fought battles for the integrity and autonomy female athletes do enjoy is not threatened. Dashper (2007) argues that "without accompanying wider changes in gender norms and expectations" (p. 213), sex integration in sport alone is not enough. The debate of sex-segregation in sport is one example of what Birrell (2000) would note approvingly as the lack of a unifying feminist theory about culture in general or sport in particular. Rather, feminism must take in account "the multivocality of current thinking within the theoretical range that can be generally referred to as feminist theory" (p. 62). As discussed above, much feminist academic and activist work in sport has focused on issues of participation. In the liberal feminist tradition, the imperative is to remove barriers society has erected that restrict their equal participation in society. However, Birrell (2000) cautions against liberal feminist traditions that advocate women be allowed to take their equal place alongside men if 
they neglect to incorporate a fundamental critique of the ideological structures that maintain the gender hierarchy. Cahn (1994) suggests that lurking beneath the surface of the debate about women's athleticism is the "nagging question of power" (p. 208). Cooky et al. (2013) interrogate the concept of "level playing field" as the end goal - specifically in terms of the justification for gender testing - as a myth that "reproduces other forms of inequalities, such as racism, classism, ableism, and others... sport is not, and arguably has never been, a level-playing field" (p. 50).

Even well-meaning advocacy work around issues such as increasing the number of women coaches in sport engage in discourses that normalize a masculinist gender ideology. The argument that women bring a "nurturing sensibility" to coaching, are more cooperative, communicative and even-tempered in constitution and who would create safer environments for athletes reifies an essentialist construction of gender as innate and biological (Gall, McIvor, \& Peeler, 2015; Yiamouyiannis \& Osborne, 2012). Norman (2010) problematizes the argument that considers "the underrepresentation of women coaches as an individual problem: women possess less intention, interest and efficacy to become head coaches" (p. 91). This rationalization of inequity underscores a methodological problem with positivist and postpositivist research that doesn't take into account the political, cultural and historical context of sport (Markula, Grant, \& Denison, 2001). Issues of pay equity, access to childcare and few opportunities for advancement must be considered as institutional barriers keeping women from progressing in coaching and leadership positions.

In her qualitative analysis of media representation of female athletes, Duncan (2006) advocates that scholars must interrogate the "appearance of equity in sport journalism" (author's italics, p. 248) that reaffirms the existing gender order. She goes on to suggests that feminist sport media research must move beyond textual analysis to avoid replicating past work that 
highlights commonplace themes while yielding little new data. Duncan advocates instead for studies that are more nuanced, that strike a balance between theorizing and data, for research that "takes into consideration the intersections of social relationships such as race, ethnicity, gender, sexuality, class, ability and so on" (Duncan, 2006, p. 249).

Despite Duncan's (2006) warning, I argue that content analysis of sport media discourses can provide insight beyond an identification of sexualized, heteronormative imagery. In earlier unpublished work (Gall, 2016), I interrogated the media success of the FIFA Women's World Cup as a pseudo-progressive discourse, arguing the increased media attention that the tournament received in the U.S. in particular was less about legitimizing the female athlete than it was about underpinning a postfeminist discourse that feminized the 'un-American' sport of soccer. Habitually framing the women's game against the men's World Cup, comparing players skills, determination and all around athleticism and finding the male game and players wanting, American media in effect marginalized the sport as a feminine domain, thereby equating soccer and the non-American cultural values it espouses with women: different, other and alien. This agenda also ensures that the integrity of audiences for mainstream male American sports is maintained; the threat that audiences might reject baseball, football and hockey in favour of soccer is neutralized by framing it as a gendered discourse. This pseudo-progressive discourse, heralding increased media coverage and a surprising lack of sexualized representation (still lacking racial and class diversity) constructs soccer as the natural site for women, where the nonAmerican male identity, specifically his masculinity, becomes de-constructed to be made feminine and inert. I conclude that this so-called media triumph for women's sports instead masks a xenophobic discourse and foreshadowed the anti-immigrant, anti-foreigner rhetoric made mainstream in 2016 and beyond. 
Even when gender difference isn't used to exclude women from sport, the sport industry and the media that cover it relies on naturalized constructions of gender for expressions of political and social power and ethnic and racial erasure. MacNeill (2009) analyzes the Olympic media coverage of Canadian track athlete Perdita Felicien, identifying the female athlete of colour as a "historic double threat" to sport media's hegemonic masculine order. MacNeill observes that "representations of women Olympians in visual and written texts do not exist as stand-alone artefacts of sporting culture. All texts draw on cultural codes, discourses and often metaphors and stereotypes to dialogically communicate at the production and consumption moments" (2009, p. 64). These images present complex and layered representations of gender, race and class. MacNeill argues that scholars must avoid what Tuhiwai Smith (1999) calls "imperial eyes" by employing an intertextual, postcolonial and feminist perspective to examine "how the media represent some female athletes as racialised 'Other', how cultural hierarchies are reproduced or resisted, [to begin] to develop decolonizing pedagogies for sport media literacy and media interventions" (p. 64).

\section{Gender, Sport And Globalization}

A growing number of scholars are beginning to explore sport as it is practiced around the world and to consider how sport intersects with localized social and gender norms in various geopolitical locations ( Hayhurst, Kay, \& Chawansky, 2015; Rowe, 2017; Thorpe, 2014; Toffoletti, 2017). Satoko Itani (2015) explores Japanese gender and sexual politics and the construction of female athletes in the male-dominated sport spaces of soccer and wrestling. Her research identifies a "third gender space .... small affective buffer, which is provided by the 
discourses of female sporting masculinity [that] tolerates a certain level of masculine gender expressions and the absence of heterosexual relationships" (Itani, 2015, p. 258). In this way, the normative and normalizing discourse of Japanese media creates a space for the 'unthinkable': masculine female athletes with masculine gender identities or who prefer masculine gender expressions. This space is not physical but produced when certain sets of discourse enable different ways of being woman or 'trans', which although temporary and situational, "allows athletes to express masculinity in sporting spaces without fearing that their gender identity and sexuality would be scrutinized" (Itani, 2015, p. 267). Itani suggests that the discourse of female sporting masculinity holds the promise of deconstructing binary genders and the categories of women and men as discrete:

if we celebrate the masculinity of female athletes as what it is (this doesn't mean that it is not necessary to critically analyze the expressions of their masculinity), rather than as a result of sacrifices and compromises, different ways of being women and different ways of being gendered might open up. (Itani, 2015, p. 266)

\section{Sport And Transgender Theory}

Sport relies on gender difference for its structural, ontological and epistemological foundations. Not surprisingly, sporting organizations have been slow to recognize and welcome transgender and nonbinary athletes (Anderson, 2002; Griffin, 1998; Harry, 1995; Travers, 2006) who are forced to contend with institutional barriers (Krane, 2001) and suffer harassment and abuse from other players (Travers \& Deri, 2011). Recently, women's hockey has become one of the few mainstream professional sports to include transgender players (Bennett, 2018). Jessica 
Platt, a transgender woman, plays on the Toronto Furies with the CWHL and Harrison Brown is a transgender man who plays forward for the National Women's Hockey League's (NWHL) Buffalo Beauts. The inclusion of transgender athletes signals a commitment from women's hockey to promote diversity and inclusivity across sport. As I will argue below, the results of my research indicate that this discourse contributes to the positioning of women's hockey as "pure", evidence of women's hockey virtue, characteristics that also indicate hegemonic femininity, a representation of naturalized womanhood that reinforces and legitimizes the dominant gender hierarchy of patriarchal society and ensures the continued subordination of women. Hegemonic femininity is a controversial term with some scholars arguing that it has escaped theoretical interrogation, reaffirms gender binary and, unlike hegemonic masculinity, flattens hegemonic femininity without taking into account any multiplicity or diversity in its performativity. Paechter (2018) argues for a reconceptualization of hegemonic femininity that rejects the limitations from being defined "other" to hegemonic masculinity, that takes into account power structures within the gender, that conceives dominance as unattached to certain bodies and that considers how hegemonic gender can be performed by different gendered bodies in ways that are not necessarily problematic. In this way, the inclusion of transgender athletes in women's hockey is justifiably celebrated as progressive and actively rejects constructs that uphold gender difference. I argue that within the larger discourse of hockey, that is the hegemonically male discourse of hockey, that framing women's hockey as "pure" and inclusive, indicates the opposite: that men's hockey is neither of these things. Rather than inspiring inclusivity, I suggest this discourse may instead reinforce a gender binary and gendered hierarchy, not between male and female, but between CIS-gendered male on one side and everyone else on the other. 
Transgender theory provides a valuable framework to interrogate issues of constructed gender identity that can:

encompass[es] and transcend[s] feminist and queer theory by explicitly incorporating ideas of the fluidly embodied, socially constructed and self-constructed aspects of social identity along with the dynamic interaction and integration of these aspects of identity within the narratives of lived experiences. (Nagoshi \& Brzuzy, 2014, p. 432)

Crenshaw's (1990) concept of intersectionality embraces the rich, complex multiplicities of lived experiences and considers social positions as constructed and relational (Yuval-Davis, 2006). Intersectional transgender scholars consider the interrelationship of race, class, gender and ethnicities as self-defined and changeable. Connell and Messerschmidt (2005) suggest that multiple hierarchies exist within gender, related but separate to the patriarchal subordination of women. The argument becomes if we can consider masculinity as dynamic, rather than unmovable instrument of oppression, this allows for the possibility of change "including democratizing gender relations" (p. 20). Of course, as these authors, and Judith Butler (1999) herself admits, sexual difference has political value, a way to gather the oppressed under one gender umbrella to agitate for social change. The cost, however, in terms of reifying the system of oppression as somehow "natural" does not take into account the intersectionality of multiple oppressed social identities that ends up fracturing whatever alliance may be established. The positioning of women's hockey as inclusive must be examined through an intersectional lens to consider issues including the lack of racial diversity in elite and community teams as well as the risk of further marginalization that works to solidify men's hockey as hegemonically masculine and dominant. 


\section{Post-structural Feminist Theory}

Post-foundational critical theories such as post-structuralism, postmodernism and intersectionality have pushed feminist sport studies to look beyond issues of participation. Cole's (1993) Foucault-influenced work is, in my opinion, foundational in this pursuit and is as relevant today as it was over two decades ago. She argues:

sport is a discursive construct that organizes multiple practices (science, medicine, technology, governing institutions, and the media), that intersect with and produce multiple bodies (raced, sexed, class, hetero-sexualized, reproductive, prosthetic, cyborg etc.) embedded in normalizing technologies (classification, hierarchization, identity, production) and consumer culture. (Cole, 1993, p. 78)

Birrell (2000) identifies Cole as a "major visionary" (p. 69) for the post-structural feminist cultural studies movement in sport. Post-structuralism considers the way institutional and cultural discourses construct and deconstruct meanings and identities that maintain or challenge power relations. This provides agency to the subject, to understand: "existing power relations and to identify areas and strategies for change" (Wheedon, 1997, p. 41) even while operating within these same systems. Gesturing towards materialism, Birrell notes Foucault's concept of surveillance and discipline as an evocative framework to consider the production of power arising from the constraints, manipulations and control enacted on the athlete's body. Feminist scholars must then examine the ways that sport disciplines the female body through diet, strenuous activity and singular focus that "anchors women into a discursive web of normalizing practices" (Markula, 2003, p. 87) and reinforces dominant hegemonic power structures. This is 
particularly true for women playing hockey, a physical game that necessitates body contact even as that contact is limited and denied by institutional barriers.

Pirkko Markula (2003) explores sport as a transformative practice that allows women to resist its power relations, even as they choose to operate within them. Markula considers the implications of Foucault's technology of self in this endeavour, the processes and discursive practices individuals willingly engage to transform themselves into ethical subjects that follow, and yet also problematize, the codes that constitute their subjectivity. This counters the idea of top down power dissemination from institutional structures, as though the subject struggles with ideological determinism that hides her inner truth. Markula describes the utilization of this theory as a directive for research that starts with the individual rather than institution and focuses on the individual first problematizing codes of sport and the resulting practices that come from the problematization. Rather than slave to the immutability of social codes, technology of self indicates the individual chooses to adopt those codes or rules and make them their own. This should not be mistaken with a kind of humanistic freedom of choice as though existing outside power structure. Markula argues that while "Foucault directs our attention to localized individual practices, he does not advocate individualism... his anti-essentialism allows simultaneous focus on the effects of power relations and the possibilities of their transformation" (2003, p. 97). This repositions the idea of resistance as an individual transformation rather than the liberal feminist goal of systemic change of social power relations, which Foucault would argue, becomes reconstituted as other (better?) power relations. There is no emancipation from power "it is present in every relationship and the task, rather than getting rid of power, is to consider how it is used through sport and physical activity" (Markula, 2003, p. 97). 
Markula argues that while technology of self doesn't indicate specific sporting practices to be practices of freedom, it nevertheless provides a framework for inquiry. Technologies of self can be transformative and transgressive but this must take into account more than change wrought through physical exertion. An athlete who diets and works out to transform her body is still operating within the limits created by the dominant disciplinary sporting practice. Markula argues feminist sport scholars must also consider Foucault's concepts of ethical self care (how power is used ethically; aesthetic self-stylization (an ongoing process of invention and reinvention of the self); and, most importantly, critical self-awareness:

an individual woman must be aware of the limitations of herself before she is able to problematize her present condition. A physically active woman, then, must become aware of the limitations of discursive femininity and the athletic self in order to reinvent herself (Markula, 2003, p. 104)

Markula sees the researcher, not just interrogating or documenting an athlete's technology of self as a transgressive practice. Instead, she argues, the sport researcher has an ethical responsibility to encourage and initiate athlete's self-reflection and critical assessments of sporting practices and performance standards, as "practices of freedom are conscious, critical problematizations of the boundaries of the female athletic self' (p. 105). This is an important consideration for future research into professional women's hockey, particularly in light of player unionization and boycott; research is needed to support the activities of athletes who have already begun to critically problematize institutional barriers.

Markula (2003) warns this athlete-led research can be conducted only when scholars "engage in an active dialogue with the sport world" (p. 105). Some scholars (Cronin \& Armour, 2015; Clark, Ferkins, Smythe, \& Jogulu, 2018) follow a hermeneutic phenomenological 
approach to sport research to offer "an understanding of how one's subjective encounter can reveal much about the shared knowings, meanings and experiences of participation in sport in our society" (Clark et al., 2018), arguing that research must be conducted not just of the body but from it as well (Allen-Collinson, 2011). Itani (2015) describes how for "third gender" athlete participants, the available discourse was as fragmented, slippery and incoherent as the performative identity categories so that the "dialectical process of 'being-named' and 'selfnaming' is a crucial task" (p. 265-266). Itani cautions us to remember that intersection is not about a "dominant-centric additive of the marginalized, but a destabilization of the centre - a deconstruction of women, men, sport, nationalism and colonialism" (p. 264-265). Methodologies must mine the homologous centre for diversity and make visible the experiences of athletes to help "deconstruct the normative and normalizing discourses of women and female sporting masculinities" (p. 265). These methodological approaches begin to address the need for more scholarly self-reflexivity and for providing female athletes with an important opportunity for expression. The same theoretical underpinnings that destabilize the centre in terms of gender construction, nationalism, colonialism as discussed above are equally necessary to consider for any audience reception research: research that allows a voice for the silent/invisible audience for women's sport is imperative.

This is fundamentally political work that seeks to challenge gender, racial and class inequities on and off the field and, in this research, on and off the ice. Even those who do not watch or care about sport cannot escape its pervasive association with core cultural values in advertising, in political discourses, in communities and even in city-wide shutdowns for celebratory parades; excluding certain bodies and identities from these discourses reaffirms their exclusion from non-sporting contexts. Conversely, sport has massive potential to be harnessed to 
agitate for positive social change. Colin Kaepernick taking the knee to protest anti-black racism (Rorke \& Copeland, 2017) for example, and the recent win of the U.S. women's soccer team in the 2019 FWWC when thousands of spectators in the arena burst into chants demanding "equal pay", a discrimination suffered by women in many workplaces, not just on the soccer pitch. Here, the issue is stripped down its core about worker value, with no less than the American president suggesting that athletes' pay should reflect revenue they generate. Despite earning roughly $\$ 30,000$ less than their male colleagues, women's soccer has generated more total revenue that the men's game (Bachman, 2019). This lays bare the hypocrisy that Meehan (2005) identifies in audience ratings; a gendered discrimination that supports ideological power structures while contradicting the structural requirements of capitalism. In this research, the lack of an audience for professional women's hockey has real implications, not just for the women players who are denied a living wage but for the exclusion of all non-CIS male gendered people from a socially relevant discourse.

\section{Women Sport Women Audiences}

The audience for women's sport must not be conflated with the female audiences for sport: perhaps against instinct, the two are not interchangeable although often positioned as such (Farrell, Fink, \& Fields, 2011). Toffoletti $(2012,2017)$ explores female sport fandom and the everyday uses women have for sport media within the framework of critical constructivism that recognizes her commodification by global sporting institutions. While much of her work focuses on female fans of male sport with a particular view on transnationalism, Toffoletti explores how female sport fans utilize social media to build audiences for women's sport and create dialogue 
to counter diverse geographical limitations. In her doctoral dissertation, Farrell (2006) investigates the reasons why women will watch men's basketball but will not watch women's basketball. The findings of her qualitative research indicate that women don't find pleasure in sport media but instead see it was a way to spend more time with family, or as a strategy to connect to or meet with men or enhance the relationship with their mates, males who watch men's sport nearly exclusively. Sport viewing, set up by, experienced with, and filtered through men, becomes an exercise in male hegemonic dominance. Conversely, Bruce (1998) writes about an audience eager to watch women's basketball only to be frustrated by coverage that trivializes player skill and misrepresents serious gameplay. While some read the preferred meaning of the text "oppositionally" (see Hall, 1980 discussed below), "deconstruct[ing] the preferred meaning before reconstructing their own positive interpretations" (p. 389), Bruce identifies this reading against the grain as harder work than watching men's sport where preferred meaning and cultural values are more in line. It may be more difficult but there is little choice for female sport viewers. As Phillips (2018) argues, broadcasters may have identified the economic value of women audiences as commodities but they have worked to subsume them into the established male sporting audience rather than build a distinctive product from scratch. Whiteside and Hardin (2011) find that domestic gender roles leave minimal leisure time available for women to watch women's sport. Burdened with the majority of domestic work, women's necessarily constant multi-tasking disrupts viewing patterns leaving them unable to commit to the emotional labour required of the sport audience. Interestingly they found women instead to be willing to commit to "time-compressed sporting events such as the Olympics during more convenient times for leisure, such as in the evenings after domestic obligations are done" (Whiteside \& Hardin, 2011, p. 140). 


\section{The Olympic Sport Audience}

As discussed above, the Olympics is no mere sporting event. It is a massive media juggernaut watched by millions of people around the world (Billings, 2008; Sugden \& Tomlinson, 2012; Wenner \& Billings, 2017). Olympic broadcasts produce an ideologically heavy text critically examined by scholars for its representations of nationalism, in flags, costumes and pageantry, country against country competition, forming and performing national identity (Bernstein \& Blain, 2003; Vincent, Kian, Pedersen, Kuntz, \& Hill, 2010), almost always aligned with gendered athletic characteristics and accomplishments (Greene, 2016; Lenskyj, 2012; Lindner, 2012). Wheaton and Thorpe (2018) identify a diminishing economic viability from the ageing demographics of the traditional Olympic audience and the intense multiplatform competition for the attention of the sought-after youth viewer as the driver behind the IOC's consideration and acceptance of more youth-favourable sports such as snowboarding and the possible inclusion of E-sports in the 2024 Games. It remains to be seen if exaltation of nationhood will hold appeal for the global digital citizen.

Margret MacNeill (1996) references Jhally’s (1984) “spectacle of accumulation” to describe the political economy of television rights and Olympic broadcast audiences as commodity, gathered, packaged and sold to advertisers; and "spectacle of legitimation" to describe the material production components that Olympic broadcasters use to "encode messages supporting dominant ideological power structures. Rather than distinctive strategies, the spectacle of legitimation and spectacle of accumulation coordinate and loop back on each other. Producers highlight specific sports, use state of the art technology and write narratives that 
construct and reconstruct national identity. The goal is to increase viewership to make a more valuable commodity to sell to advertisers and commercial corporations invested in the perpetuation of the dominant hegemonic status quo. MacNeill is one of the few scholars to critically analyze Olympic hockey as a television text, exploring "encoded" messages about dominant ideological norms that audiences presumably accept, reject or negotiate. In order to explore how audiences “decode” meaning from Olympic women's hockey media text and particularly how that relates to watching or not watching professional women's hockey, I turn to cultural studies and the theoretical, epistemological and ontological foundations of audience reception research.

\section{Audience Research}

Much like hockey, audience reception research is structured as a network of teams, each with their own distinctive theoretical foundations and research traditions. Also, like hockey, audience reception research has seen its fair share of brawls, conflicts erupting over methodology (qualitative vs. quantitative), academic disciplines (social sciences, humanities, cultural studies) and definition of the audience (active, passive, implied, dupe and non-existent). Noted academics characterize audience reception research at either a crossroads (Livingstone, 1998) or in need of revival (Gray, 2017). Jonathan Gray (2017) writes of audience reception research red-lining, threatened by empirical studies rendered "at worst wrong, at best dated" in the face of Netflixstyle big data, and by audience studies in the critical cultural tradition that "has variously dwindled and become so hyper-specialized" by the preponderance of fan studies that the two terms have become synonymous. Now, he tell us, "is the time for a revival and maturation of 
audience studies in the critical cultural vein" (p. 80) that looks beyond fan studies to the casual viewer and then even more specifically to those who don't like or, even don't watch programs or other media content. Consideration must also be paid to the audience beyond Cubitt's "politics of the sitting room" (1985, p. 178) to mobile media technologies, shared viewing in public spaces, and the online studio of the consumer/prosumer around the world. Despite the clarion call, the unmistakable tone continues to reflect uncertainty at best, and crisis at worst, for the advancement of meaningful study into how audiences engage with media texts and how they make meaning that illuminates, obfuscates, reinforces or challenges their sense of themselves within the world.

Livingstone (1998) suggests this turmoil is productive, that the crossroads is less about a crisis than a call for a "a multi-disciplinary convergence in which the major splits within media and cultural studies might be resolved" (p. 195). The way forward indicated by Livingstone $(2013,2019)$ is through an integration of media and cultural studies research traditions, research that considers the "implied audience" theorized from text-based studies and production studies and connects this to audience studies. Others (Gunter, 2000; Morley, 2006; Schrøder, 2013) indicate integration as a commitment to methodological pluralism. To conceptualize a softening of the edges that have defined and divided audience research, it is important to first explore the development of audience studies as an exercise in opposition.

\section{Social sciences.}

In 1896, an audience in Paris sits down to a screening of the Lumière brothers film L'Arrivée d'un train en gare de La Ciotat. The position of the camera means that as the train pulls into the station it gets closer and closer to the lens until it exits camera right. The audience, 
unused to this new technology, imagines the train will burst beyond the confines of the screen to run them all down causing a stampede out of the cinema. This famous story is likely an urban legend, at least according to Loiperdinger and Elzer (2004) after conducting a detailed frame by frame analysis of the film and undertaking an historical investigation into the event and the promotional and press material around it. Immortalized in film history textbooks, the legend of audience panic at the Lumière screening persists, along with the assumption that media texts have great power to manipulate and therefore pose great danger to spectators.

Audience studies began in the social sciences to address concerns over mass media and the vulnerability of its audience, passively unaware, and at the mercy, of the political messaging that was World War I propaganda. Early media theorists (Lasswell, 1927; Lippmann, 2017) argued the marked social changes as a result of industrialization and urbanization had left fragmented and isolated individuals unable to withstand the effects of this "onslaught of subversive mass media messages" (Bryant \& Zillmann, 2009, p. 10). The "hypodermic needle" or "magic bullet" theory (Lasswell, 1927) presumed a linear association between content and effect wielded with dubious authority by masterminds seeking to control people's thoughts and behaviour. Lazarsfeld (1944) tested these theories in a study meant to examine the link between voting patterns and propaganda; his conclusions demonstrated interpersonal relations to be more influential on voter choice than any media effect. He instead theorized a "two-step communication" model arguing that audiences are influenced by opinion leaders, "individuals who exert an unequal amount of influence on the decisions of others" (Rogers \& Cartano, 1962, p. 435), who are in turn influenced by media texts they have consumed and contextualized.

Lazarsfeld's administrative approach to audience research stood in contrast to the work being done by European scholars at the Frankfurt School who were developing a critical theory 
of the cultural industries. From their Marxist perspective, they considered the political implications of mass culture that imposes media texts on audiences to dull senses, rob imaginations, take over brain functions and willingly labour in the profit-driven cultural industry. As articulated by Horkheimer and Adorno, two key theorists from the Frankfurt School, the "culture industry" signifies the process of the industrialization of mass-produced culture and the commercial imperatives that drive the system that "perpetually cheats its consumers of what it perpetually promises" (Horkheimer and Adorno, 1972, p. 139). Lazarsfeld invited Adorno to collaborate on a Princeton University radio project in order to establish a landscape that would allow for a convergence of the administrative and critical theory approaches to the role of media in the social system. Epistemological and political differences between the two (scholars as much as theories) left the project in failure (Slack and Allor, 1983).

Media effects researchers went on to consider a more complex, nuanced view of the audience and challenged the idea of the purely passive viewer. Developed in the 1940s, the uses and gratifications model measures a somewhat more active viewer in terms of their choice of program or content that meets or gratifies a specific or general social or psychological need. Early studies were more descriptive than analytical, classifying audience responses (gratifications) into set categories (Berelson, Lazarsfeld, \& McPhee, 1954; Katz \& Lazarsfeld, 1955; Lazarsfeld, Berelson, \& Gaudet, 1968; Merton, 1949) but the focus remained on the effects or what the media did to the audience and why certain audiences would choose or be drawn to certain texts. Criticisms of this approach were both methodological (a reliance on selfreporting) and theoretical (a lack of critical examination on the origin of the social need being fulfilled and no representation or analysis of the constraints of the text), (Ang, 1995; Chaney, 1972; Elliot, 1974; Swanson, 1977). Further development of uses and gratifications re- 
positioned, contextualized and re-animated the viewer to examine not only what media did to the viewer but what the viewer does with the media (Katz, 1959). Katz and others examined the diversity of uses viewers can find for media, from entertainment and pleasure to distraction and education, and sought to understand how social situations can filter media influence. In this formulation, the audience is somewhat active to choose and use media texts and, even in Katz's functionalist iteration, do so in a multiplicity of ways. However, its reliance on individualism neglects to consider the social context of either audience, text or social institution; this left uses and gratifications open to criticism from critical theorists, particularly from the Centre for Cultural Studies in Birmingham.

\section{Cultural studies.}

With its focus on the everyday, low culture popular texts, the audience research conducted from Birmingham's Centre for Cultural Studies (BCCS) in the 1980s also considered how texts act to legitimize power structures and support a dominant ideology that works to normalize meanings of class, race, gender, policies and social values as though by 'common sense'. Hall (1980) theorized audience reception research as a way to understand how audiences make meaning from mass media texts through interpretation. He argued that audiences decode messages in a variety of ways that either support the "preferred" or dominant message encoded in the text, or require a modification of encoded meaning to reflect individual positions, experience and interest in a "negotiated" reading. Or, audiences may reject encoded dominant meanings in an "oppositional" or resistant reading (Hall, 1980, p. 101). Hall's theoretical work in audience research was developed, in part, in opposition to the "passive" audience of early social science research. This cultural studies model considers the audience to be "active" in their 
engagement with the text, how they "decode" the messages that are "encoded" in mass media texts that support dominant hegemony.

Where uses and gratifications research, even in its most developed form, allows for the audience to assert autonomy in terms of what they choose to watch or not watch, the theorized process is linear and close-ended. The meaning or message of the text is delivered from the producer to the individual audience member who exhibits a reaction, or even non-reaction, to its meaning. At BCCS, Hall argued instead that mass communication was a more complex process that allowed for a variety of responses from the audience/receiver depending on context, experience and social background. Production practices create television texts that act as "signvehicles", encoded with meaning and distributed to audiences through broadcasting structures; texts must be decoded by audiences and translated into social practices to complete the communication process. Each of the four stages of the communication process, (production, circulation, use and reproduction) are connected yet remain autonomous, distinct and therefore possible to disrupt. Unlike previous media theories that privileged interpretations of the text, Hall considered the audience an active contributor to meaning making. Pre-dating Livingstone's call for convergence, Hall advocates for a careful textual analysis to determine the preferred reading and an application of codes to audiences that might then either accept ("dominant-hegemonic") or pick and chose ("negotiated") or reject ("oppositional") encoded messages. For Hall, the audience decoding depended on their position in the social power structure so that how a text is understood and valued depends on social situation. As Morley (2006) explains "the elements of comprehension and evaluation will be inevitably intertwined — with some kind of interpretations dismissed by more powerful others as merely 'misunderstandings"'(p. 110). 
Hall's theories were paradigmatically and methodologically enacted in Morley and Brunsdon's (1978) work, first with a textual analysis of the current affairs program Nationwide and then followed up with Morley's (1980) empirical analysis of the Nationwide audience using the encoding/decoding model. Despite the social determinism grounding the assumption of linearity that audiences will decode text as a consequence of their socio-economic status (either in accordance with or in opposition to or a struggle between), the Nationwide Audience Study was foundational both for its initial semiotic and ideological analysis of the text to uncover encoded messages of nationhood and for the wide variety of decoding done by audiences in amongst and within set socio-economic groups.

By the 1980s, audience studies had developed into a substantial discipline in cultural studies and, according to Gray and Lotz (2012), rejuvenated television studies. Studies were conducted that focused on marginalized audiences and how they interpreted popular television texts, including children and women viewers. Buckingham (2008) addressed the debate between cultural studies and media effects in his research to determine the impact of televisual violence on child and youth audiences. While acknowledging the political implications of media effects based research that allowed politicians to blame television for socially based issues at the root of violence in children's lives, Buckingham argued that the emphasis of research must be less on how media effects viewer behaviour and attitude than on how viewers interpret, negotiate and distribute meanings. Research must also consider the diversity of possible meanings that children and youth can find in mainstream television texts, including pleasure from questioning and ridiculing narrative strands and characters.

Lewis (1991) argued against scholars who read television texts for political and social messaging without examining audience interpretation although, particularly in his 1992 work 
with Jhally, Lewis argues that audience interpretation and autonomy does not mean that television texts are ideologically weak (Jhally \& Lewis, 1994). Their ethnographic research with audiences of the Cosby Show, a popular 1980s sitcom featuring an affluent black family, argued that while black and white viewers found pleasure in the Huxtables and their middle-class stories, they interpreted the text in different ways and for very unexpected reasons. White viewers considered the show to be an affirmation of racial equality, as though systemic racism can be fixed by individual (black) success. Black viewers, on the other hand, while appreciating the representation of black lived experiences beyond the stereotypical working class and often foolish sitcom character, critiqued the socioeconomic status of the characters for being unrealizable to them. Far from aspirational, the text instead disassociates black viewers and their lived experiences. While acknowledging the polysemic nature of the television text that offers a variety of opportunities for diverse audience interpretation, Jhally and Lewis (1992) argue for the need to acknowledge the ideological work of the text, in this case celebrating the financial achievements of one family without acknowledging how capitalism perpetuates racial discrimination. Of equal importance is the reminder that audience interpretation must not be assumed.

For feminist scholars, women audiences were poorly served in both administrative audience research and cultural studies audience reception. Television texts associated with female viewers were denigrated and deemed unworthy of academic attention, particularly from cultural studies audience work focusing on serious television texts and their implied serious (male) viewer. Feminist scholars such as Fraser (1985), McRobbie (1978) and Press (1990) began to consider the private sphere of the domestic home as porous and therefore subject to the 
hegemonic gender hierarchy. Audience reception research becomes not just about the program text but who controls the viewing technologies (Gray, 2006).

\section{Feminist studies.}

The association between feminism and qualitative audience reception research has a long history beginning with Herzog's (1941) study of female listeners of radio daytime soap operas. Unlike future studies that sought to recuperate the soap opera from its low culture positioning, Herzog trivialized the genre and its female listeners as innocent and unworldly. Nevertheless, her work is noteworthy in its intention to illuminate the role of media in the everyday lives of ordinary women in the domestic space. Herzog argues that women escape the drudgery of their existence through the vicarious adventures of soap opera heroines. The study illuminates the ups and downs, the triumphs and frustrations of everyday domestic life, a deeply gendered experience that had been ignored as a valuable research target. Herzog's work defined the domestic sphere as a legitimate and cohesive domain and its female inhabitants as active consumers of media texts. This would become important in future decades when feminist theoretical and political action demanded a recognition of women's unpaid domestic labour. Radway (1984) contextualized and legitimized readers of the romance novel, a previously disparaged "trash" sub-genre of the heavily gendered melodrama. Radway also recognized the process of consumption and meaning-making as a complex social event allowing the equally complex female reader to both escape from and protest the restricted role allowed to them under a patriarchal social structure.

As Radway did with her work on the reader of romances, feminist audience researchers have conducted ethnographic studies of audience engagement with so-called "low-cultural" texts 
gendered as feminine, specifically the soap opera to, in part, "celebrate the specificity of women's pleasures and to revaluate them against the grain of male denigration" (Geraghty, 1991, p. 299). Before Morley, Dorothy Hobson (1982) conducted ethnographic audience research with viewers of the British soap opera Crossroads in their own homes and settings. Hobson theorized that while the media text may be encoded with hegemonic messages, viewers appropriate the text and contribute to it through their own personal experiences. For the audience, "there is no overall intrinsic message or meaning in the work, but it comes alive and communicates when the viewers add their own interpretations" (Hobson, 1982, p. 170). For Hobson, pleasure, certainly the necessary prerequisite for viewers taking the time to tune in repeatedly, can be found not in the text but in what the audiences $d o$ with the text.

Ien Ang (1985) considered the ways Dutch audiences make sense of and derive pleasure from watching the evening soap opera Dallas, a low culture text dismissed by many cultural critics for perpetuating "American cultural imperialism" (p. 2). Disrupting "the arrogant ideological work of distinction that propped up this social hierarchy of value" (Ang, 1985, p. 4), Ang discovered an audience actively extracting and constructing meaning from the text in a multiplicity of ways. Most of her respondents described being entranced by the "tragic" emotional relationships between the characters, the continual cycle of fracture and reparation, pleasurable in its tantric incompleteness. This emotional engagement or affective mode of pleasure can only be accessed by the audience through cultural competency, that enriches ordinary, everyday emotional activity with melodramatic value that would otherwise be seen as trivial, an "intimate level of feeling" (Ang, 1985, p. 3). Ang identifies multiple decodings to the encoded text but argues the melodramatic imagination that allows the predominantly female viewer to find pleasure in Dallas results from the social positions held in that particular society; 
conventional representations of femininity outweigh whatever cultural differences exist between Dutch and American audiences. Pleasure in the text depends on this recognition and in doing so, re-affirms gendered social norms, both in viewing and outside the text in conversations with workmates, friends and family.

Ang identifies other decodings such as "ironic disposition" where viewers use humour to create a distance between themselves and the text, allowing them to find pleasure in the characters and narratives while rejecting the discourse that normalizes the "cultural imperialism" of the text. This, in Foucault's (1981) argument, makes the object graspable by raising the viewer to a superior position to find humour in the 'low quality' of the media text. As she re-visits her earlier work, Ang (2007) marks the growth of the "ironic decoder" with the proliferation of postmodern self-reflexivity in many Western television texts that subsume the sentimentalism of soap opera/melodrama. Far from being swept away, audiences of 21 st century soap opera texts, most commonly reality/docusoap series, "are engaged in critical viewing of the attitudes and behaviour of the ordinary people in the programmes, and the ideas and practices of the producers of the programs" (Hill, 2004, p. 9). Yet, even in its current "reality" iteration, a soap opera or docusoap like any of The Real Housewives franchises continues to be 'gynocentric', appealing to a largely female audience, and derided for presenting "an over-dramatic, under-rehearsed presentation of trivial dramas blown out of all proportion to their importance" (Geraghty, 1991, p. 1). These media texts are still ripe for 'oppositional decoding' to reveal "subversive, potentially resistant feminist ties that undermine the very conservatism that the show perpetuates" (Levy, 2018, p. 370). Ang's "melodramatic imagination" becomes less emotional realism than inspiring affective mimicry as emotional outbursts perform and engender feminine rage. 


\section{Soap Opera And Sport}

Given the expansive audience research into the soap opera as a gendered engagement with a gendered text, can these theoretical foundations contribute to a cross-fertilization with audience engagement with sport as a specifically gendered media text that performatively constructs masculinity, or, what McKay and Rowe (1997) refer to as "masculine melodrama" (p. 76)? O’Connor and Boyle (1993) argue that sport media exhibits many of the same characteristics of soap opera. Built around an ensemble cast of characters with multiple story strands following a continuous narrative, an indeterminate ending and frequently scheduled regular broadcasts, sport media texts, like soap opera, "invoke similar structures and feelings of sensibility in their respective [highly gendered] audiences" (p. 109). While the soap opera's “open-ended, slow paced, multi-climaxed structure” (Modleski, 1982, p. 98) doesn’t appear to immediately lend itself to sport, O'Connor and Boyle argue that with its multiple cameras and frequent starts and stops that isolate characters and compress time and space, mediatized sport follows the same institutional codes and conventions as soap opera. The continuous open-ended soap opera text is like ongoing league championships, each individual game never fully closed until the series' final, at which point talk immediately turns to preparations for the next season. Sport media appeals to audiences much as soap opera, for the "openness which allows for multiple and complex identifications with players/[characters]” (O’Connor \& Boyle, 1993, p. $113)$.

Others scholars consider how media coverage creates particularly masculinized melodramatic meta-narratives of sports-related events. Stories of owners and players locked in 
brutal contract negotiations, athlete fights, arrests, retirements and comebacks provide a "behind the scenes" perspective to deepen on-field drama (Cole and Denny, 1994; King, 1993; McKay, 1993; McKay \& Rowe, 1997; McKay \& Smith 1995; Muller, 1995). Dalbir S. Sehmby (2002) identifies televised pro-wrestling positioned within the tension of a masculine-feminine narrative form, satisfying "feminine" soap opera criteria with displays of excessive emotion, recurring characters, plot twists and turns while meeting the demand for a highly "masculinized" performance of aggression and violence (see also Jenkins, 1997 and Pratten, 2003). The audience finds pleasure in the contrivance of wrestling as performance, enjoying a "position of privileged knowing" (Sehmby, 2002, p. 34).

Ang (1985) suggests that audiences find pleasure in soap opera through recognition, "the possibility of identifying oneself with it in some way or other, to integrate into everyday life“ (p. 20). O'Connor and Boyle argue that familiarity with players, their personal lives, their stats and stories, told in magazines and tabloids, creates the opportunity for the audience to feel a personal connection. Production techniques such as the close-up are utilized in both sport (Whannel, 1992) and soap opera to further establish feelings of immediacy and recognition. This allows audiences to further invest in the dramatic conflicts and resolutions played out. Pleasure comes from recognition and identification but also from the cultural competency to read codes about gender and identity. More than other genres, sport and soap opera celebrate and award traits that are representative of gender norms and heteronormativity:

Televised sport affirms fantasies and realities of power, control and autonomous activity. Soap opera attempts to address and construct a female audience in terms of tolerance and passivity clustering around three areas of particular concern to women: the family, motherhood and romance. (O’Connor \& Boyle, 1993, p. 114) 
This is not to suggest that men don't watch soap operas, or that there are no women viewers for sport; the argument is that textually, the central construction of gendered identity falls along predictable lines. Viewers, regardless of gender, find pleasure in this recognition. How then to understand the excessive emotionalism of the sport audience as anything other than a contradiction of its own gender stereotype? How to solve the paradox of the constructed hypermasculinity of the hero athlete with the hyper-emotionalism of male sports fans crying, smashing TVs or punching holes in walls over bad outcomes while others jump, scream and cry with joy at their team's victory?

\section{Affect Theory}

I argue that the excessive emotionalism of the sport audience is less a function of the idiosyncratic proclivities of individual fans and more a result of the way affect is created, embedded and circulated in sport media texts. Richard Pringle (2015) turns to affect theory to explore the paradox in his own sports viewing between his role as academic critic of the Olympics and the very real pleasure he experienced watching the 2012 London Games, almost as though in spite of himself. While scholars have focused on how sport media texts symbolically and discursively construct social identities and reaffirm ideological power structures, queries have centred on audience interpretation as a cognitive process. Pringle contends this approach fails to account for the overwhelming popularity of sport and the pleasure audiences clearly derive from watching and, most importantly, how that pleasure can obfuscate the workings of power and "encourage sport fans to uncritically accept the political messages that are embedded within the sport media" (p. 117). Pringle argues that the visual representation of charged 
emotional moments so common in Olympic broadcasts, "an embrace, a smile, a tear, an ignition of elation, a hug, a kiss, a shake of disappointment, a supportive touch" (p. 126), effectively circulates the same emotion to the viewer while commentators' dramatic descriptions of athletes' and crowd emotions work to discursively construct a narrative of sporting performances that "provide coherence and meaning to the Olympic representations" (p. 129). Pringle and other scholars reach beyond the cognitive, taking an "affective turn" (Clough, 2008) to understand the relation between audience and text as an emotional exchange. Anna Gibbs (2011) asks: "what contribution, then, does affect make to the generation, hold, and sway of ideas in contemporary society? In other words how are affects implicated in belief' (p. 255)?

In their introduction to The Affect Theory Reader, Seigworth and Gregg (2010) poetically position affect as in the midst of "in-between-ness: in the capacities to act and be acted upon ... visceral force(s) beneath, alongside, or generally other than conscious knowing, vital forces insisting beyond emotion" (author's italics, p. 4). Gorton (2009) recognizes the difference between affect as "firmly rooted in biology ... physical responses to feeling" and emotion as a "sociological expression of feeling" but uses the terms interchangeably to explore "the way feeling is negotiated in the public sphere and through the body" (p. 62). Both Gorton and Sara Ahmed (2004) reject a cognitive/affective binary separating knowing and feeling; Ahmed identifies the division as an inferred hierarchy: "the story of evolution is narrated not only as the story of the triumph of reason, but of the ability to control emotions, and to experience the 'appropriate' emotions at different times and places”'(p. 3). American psychologist Silvan Tomkins enacts a "hierarchy of emotion" in his primary human motivational system, nine discrete affects categorized as either as positive/rewarding (enjoyment-joy and interestexcitement) or negative/punishing (fear-terror, distress-anguish, anger-rage, shame-humiliation, 
disgust-contempt, and the "auxiliary" affect, dissmell and surprise-startle) (Sedgwick \& Frank, 1995). These affects form the basis of human sociality, neither solely neurological nor social constructions, but "bio-psycho-social [phenomena], in which the corporeal and the cognitive are inescapably conjoined in ways which are contingent on experience" (Gibbs, 2011, p. 254).

Others insist on a theoretical distinction between affect and emotion as though places on a kind of cognitive continuum, affect positioned very distinctly as 'pre-cognitive', a knee-jerk gut instinct felt first in the body before presumably intellectual processing emits an emotion. Shouse (2005) refers to the affective competency of infants who, without language or cognitively developed expression, communicate affect corporeally through facial expressions, sounds, breathing as though innate or even primal. Massumi (2003) distinguishes affect as "an energetic dimension or 'capacity' and emotion as a selective activation or expression of affect" (as cited in Gibbs, 2011, p 251). For theorists like Deleuze, Guattari and Massumi (1987), the concept that affect works at a molecular level allows them to work beyond representation or knowledge as signification and instead explore the connections, intimacies and integration of not just media texts but media artefacts itself. Lazzarato (2006) describes this as "machinic enslavement" where "the individual and the machine are sets of elements, affects, organs, flux and functions, all of which operate on the same level and which cannot be articulated as binary oppositions - not subject/object, human/non-human, sentient/intelligible" (p. 4). Thrift's (2008) NonRepresentational Theory (N.R.T.) focuses on the pre-cognitive aspects of everyday life as a "rolling mass of nerve volleys [preparing] the body for action in such a way that intentions or decisions are made before the conscious self is even aware of them" (p 7).

For these theorists, and indeed for the purpose of audience reception studies, the question goes beyond what affect/emotion is to what affect/emotion does? In particular, how does 
affect/emotion circulate between bodies and objects and what impact does that have on us as viewers/users and citizens? Kavka (2008) refers to Grossberg's “wild realism” in her examination of reality television to indicate "both excess and irregularity, something which exceeds and escapes the ideologically grounded function of realism" that demands scholars “open up a critical space beyond the structures of signification and meaning production which have defined post-structuralist approaches to texts and technologies, including television" (Kavka, 2008, p. 26). Has the affective exchange in our mediated history spilled over into political and social discourses to, in effect, overrun cognitive perceptions? Or is it mediatization that increasingly rules all facets of social and political interactions that prioritizes affective expression and response articulated politically as feeling, as it were, over fact?

\section{Affect And Audience Research}

Gorton (2009) looks at television texts using affect as a theoretical lens to explore how producers use emotion to connect and engage with their viewers. She considers how programs, particularly from the reality genre, encode and embed emotion in text using production strategies such as music and close-ups. Carl Plantinga (1999) in his work on film and affect, argues that the face plays a vital role in not only transmitting emotion but eliciting same in the viewer: “emotional contagion is the phenomenon of 'catching' others' emotions or affective states ... when our friends laugh and smile while telling us a story, we often laugh and smile in response even if we fail to see the humour in the story itself" (Plantinga, 1999, p. 243). The close-up amplifies the affective power of the face, along with music, voices and editing, "broadcasting human emotion [which] then produces complex feedback loops between image, individual, and 
audience in which causes and effects become interchangeable" (Gibbs, 2007, p. 253). Crucially for audience research, this emotional engagement is not to be mistaken for passivity. Emotionalism or sentimentality has been assumed to "lead us away from active, cognitive engagement with the ambiguities and complexities of the real ... toward the over-simplified, the distorted, the falsified and the fantasized" (Knight, 1999, p 417); yet, feminist scholars invested in recuperating melodrama and its broadcast soap opera equivalent and "the mad, hysterical and overly emotional women" (Gorton, 2009, p. 68) who engage with them, argue against cognitive/affective tension (Mankekar, 1999) as a critique of the gendered place of emotion in everyday life. For audience researchers, the question becomes not only how do viewers affectively decode the emotional encoding purposefully placed in both the text and through production strategies, but what are the implications of that ongoing circulation of emotion once the program ends?

The idea of affective contagion requires further examination. This is more than a simple exchange of feeling, as though happiness is a thing that one has and passes it on as a piece of property. Ahmed (2004) points out that “even when we have the same feeling, we don't necessarily have the same relationship to the feeling" (p. 10). Even though a television program constructs an emotional moment with extended close ups and sentimental music, this does not necessarily mean that all viewers will experience the same emotion or catch the emotion in the same way; some will laugh, some cry and some will feel bored by the same moment (Probyn, 2005). Ahmed (2004) discounts the idea of emotions as something we "have", initiated inside the body; she suggests instead that emotions make boundaries: "it is the objects of emotion that circulate, rather than emotion as such. Emotions can move through the movement or circulation 
of others. Such objects become sticky, or saturated with affect, as sites of personal and social tension" (p. 7).

Ahmed's stickiness underscores how affects such as hate, disgust and fear, in particular, works on and through bodies suggesting:

hate is involved in the very negotiation of boundaries between selves and others, and between communities, where 'others' are brought into the sphere of my or our existence as threat.... If hate is felt as belonging to me but caused by an other, then the others (however imaginary) are required for the very continuation of the life of the ' $I$ ' or the 'we'. (p. 51)

As Ahmed interrogates systemic racism, she considers affect as it becomes what "sustains or preserves the connections between ideas, value and objects" (p. 29). In this way, audience research should consider the circulation of affects, their repetition or contagion, or as Gibbs (2011) puts it, "affects powerful enough to be transposed from one context into another more public one, where they become attached to ideas which seemed to give legitimate voice to them (p. 259). Positioning media audiences as a more active "publics" and referencing French sociologist Tardes (1962), Gibbs characterizes contagion as a mimetic communication that goes beyond the cognitive, to involve "nerves, muscles, and affects. Imitation is 'suggested' then, both by the force of habit (the force of the past) and, more immediately, by the force of the sympathy that is the response to what is suggested by other bodies" (Gibbs, 2011, p. 260). Rather than face-to-face conversation that more traditionally disseminated opinion, Gibbs considers online intimate conversation in discussion groups and other online platforms to generate imitation that increasingly expands and spreads globally: "swept through by contagion without ever coming to form an aggregated force" (p. 264). For audience research, Gibbs calls for an "epidemiology of 
affect" that maps or traces the "affect-born migration of ideas" (p. 264) to show how they spread globally, to either wither and die or bloom and propagate further imitations.

Gorton (2009) argues that television programs that have the ability to move viewers emotionally have political value, not just in terms of content, but also in the "sense of connectedness and belonging that is repeated in each episode. The repetition [of emotion] attaches them to the text; they often come to expect and even desire this engagement" (p. 94). Affectively engaging with certain media texts provides a "gateway" to craving affect in increasing intensities, setting the emotional stage for a viral affect outbreak. The strength of the affective contagion is increased by the easy and even constant accessibility to media through mobile technology. Beyond the specific texts, the way we affectively engage with the materiality of our television sets and our digital devices must also be incorporated into audience research.

\section{Affect And Mediality}

There is a precedent in audience research to focus on the media device, traditionally the television set and its technology. Morley (1986) and Bausinger (1984) focused on the television set and remote control respectively as symbols of masculine power in the home. Gray (2006) examined the VCR in terms of domestic technology and women's leisure. Spigel (1992) positioned the television as integrated in everyday life, within the physical domestic urban and suburban sphere, as glue to bring the postwar nuclear family together. Kavka (2008) sees the television set not only relaying programming to which audiences affectively engage, but as the site of affective transmission or deployment. 
Kavka focuses her affective analysis on reality television as a genre that glories in emotional excess. The "affective call" to the audience is so effective, not only because it is experienced by supposedly 'real people', but also because it is based on identification with the affective situation itself. This is an instinctive alignment to the intensity of feeling on the screen, even if the audience member has never 'felt' or expressed it the same way. Audiences not only identify with the affective situation, their feelings are engaged, amplified and made all the more real because they are shared with the others they see on the screen. This "hyper-familiarity" creates a shared affective reality that provides a feeling of comfort to the viewer: "We feel in some sense because affect moves through and across us, in palpably material form, resonating across divides that may otherwise be blocked by cognition or representation" (Kavka, 2008, p 33). Kavka suggests affect moves across a divide that is the actual TV screen itself forming a bridge or cusp.

Kavka makes reference to Brennan (2004) who argues that affect or emotions can be materially transported from one body to another by a process of 'entrainment', as though a form of psychophysiological osmosis. In this way, affect can be transmitted by touch, sight, sound or even by pheromones but also moved in and between material objects. Objects both receive and transmit our feelings and also other people's feelings about the object. The object is then evacuated and refilled with the material of a feeling that is and is not our own. This has theoretical precedent from Deleuze (1987) who argued that affectivity is a quality of things as well as people, that by transmitting or linking affective states, the thing itself becomes an affective participant, most famously suggesting that Jack the Ripper's knife edge is capable of affect (p. 97). 
For Freud (1940), affect is a bridge joining two distinct divisions between the unconscious and conscious; for James (1952), affect connects the body and psyche; for Brennan (2004), affect connects bodies. This join, cusp or bridge is a form of mediation or intermediation and as affect crosses, knowledge is produced:

affect matters, because feelings - of joy, distress, excitement - perform a function of intermediation between selves and the world of objects/others. This intermediation, the contact point of the self with objects/others which produces another kind of knowledge about the world, in turn aids relationality, which is to say that it is the glue of affect that gives our relationships their sticky existence of mattering. (Kavka, 2008, p 35).

This falls in line with Latour (1994) who considers mediation as a translation, not a neutral reproduction of meaning or information, but an active transmission of affective state. Kavka argues that mediation is already central to affect at the cusp formation or as intermediation. Technologically and materially, affect functions as a "hard-wired circuit" linking bodies, psyches and the world. So, affect that is produced at the site of the cusp formation, even if that site is a piece of technology like a TV screen, that affect is real. TV text in particular as discussed above, amplifies and increases affect with close-ups and music, connecting "real people" on one side of the screen with, as Kavka argues for reality television in particular, "real people" on the other side of the screen, bound together in a shared reality by affective glue.

If the television functions as cusp or bridge allowing the circulation of affect in, through, to and from the screen to viewer, the screen saturation of our digitally invested lives from laptops to mobile devices to wearable technologies only ups the affective ante. Audience research must take into account digital technologies and alternative viewing environments for programs and online materials. In this context, the word audience is a generalized slippery term conflating 
viewer, user and prosumer, who engages, interacts with, or even creates media texts/content. Affect theory is particularly suited to go beyond the text, regardless of platform, to engage less with what the media (or content) is or says than what the media does. As the results of this study will show, affect circulated from the sport media text of Olympic women's hockey inspires emotional and physiological reactions that create audience engagement. While affective in nature, audience engagement is deepened through cognitive processing related to patriotism and also player statistics, behind the bench politicking and negotiations. In this study, participants and respondents' call for increased media coverage of the female athletes can be understood as an attempt to create a foundation for an emotional connection to the players and the games. I contend that sport media also circulates affect disconnected from any cognitive processing as audiences' physiological responses to mediated game play provide an affective 'hit'. Conversely, as I will argue, the lack of audience engagement for professional women's hockey is a result of, not the absence of affect, but from its disavowal.

\section{Converging Theoretical Foundations}

This chapter has explored and synthesized literature from different scholarly traditions to create a theoretical foundation from which to conduct this audience reception research. Understanding how audiences interact with, interpret and find meanings in television texts requires a cultural studies foundation in audience reception research. Exploring the implications of women playing the hypermasculine sport of hockey leads to an engagement of critical constructivist scholars who study sport media and gender. Examining how feeling and emotion help to create meaning out of television text necessitates an affective turn. Feminist post- 
structuralism provides the theoretical framework to interrogates the discursive construction of gendered subjectivities. There are four key concepts underlying this audience reception research: 1) the recognition of hockey as a powerful cultural discourse that defines normative social identities, particularly in terms of gender and nationalism; 2) audiences engage with media texts in a multiplicity of ways decoding meaning that aligns with, resists or is result of negotiation with the encoded dominant ideology. Further, audiences create meaning from the absence of text; 3) affect is circulated through sport media texts to create audience engagement through emotional and physiological response; 4) broadcaster reliance on administrative ratings do not automatically reflect audience interest. In the next chapter, I will extend this intention to pluralism with a discussion of the reasoning, planning and implementation of mixed methods. 


\section{Chapter 3 Research Methodology}

I am, by nature and training, a qualitative researcher. My academic work and my documentary film work has relied on my ability to communicate with individuals and groups, to ask questions, lead discussions, and to interpret, categorize and analyze their answers and statements in order to form conclusions. The purpose of this study is, at first glance, qualitative in nature. My research seeks to explore the reasons why audiences will watch women play hockey during the Olympics but will not watch them play professionally and the implications of gendered exclusion from cultural discourse. As detailed in Chapter 1, quantitative research in the form of ratings from Numeris already tells me the audience for professional women's hockey is a fraction of the men's. Yet, as I will argue in this chapter, qualitative methods alone would not have sufficed. I required data that was both broad and rich, that indicated trends and allowed individual statements of (dis)engagement with professional women's hockey. Most critically, I did not want data from a smaller sample set to be interpreted as evidence of a lack of interest in women's hockey and further contribute to its marginalization. For these reasons, I utilized mixed methods beginning with a quantitative component in the form of an online survey, and followed up by focus groups to provide the qualitative data.

This chapter will begin by exploring the theoretical foundations for the suitability of mixed methodology in audience reception research and present the reasoning for its use in this research study. The debate in audience research methodology can be understood on a historical timeline. Traditions and paradigms are developed over time, researcher objectives, epistemologies and ontologies creating forks in the methodological roads. Early quantitative audience research may have led scholars to utilize qualitative methods in response, but it did not keep other social scientists from continuing positivist pursuits. The historical timelines of 
audience research methodologies run parallel rather than linear, occasionally intersected and yet still discrete. I review the methodological traditions for television audience research in general as well as the more specific audience for broadcast sport. In keeping with the theoretical foundations of this research project, this chapter explores qualitative, quantitative and mixed methods audience research from a critical feminist perspective. I ponder the limitations to the design and implementation of this study and identify the challenges of gathering data measuring audience affective response.

\section{A Methodological Debate}

The field of audience research is in perpetual flux (Das, 2018; Gray, 2017) abounding with academic arguments about the flow of meaning between viewer and text, the nature (and existence) of the audience, their agency or vulnerability and what it is that they do with media and what media does to them. Barker (2018) argues that debate in the field of audience research so often has less to do with specific projects or findings than with commentaries or a metacritique about "conceptions of 'the audience' and what fundamental languages, concepts and methods we can use to investigate them" (p. 132, author's italics). The traditional methodological distinction in audience research is between "positivism, empiricism, and behaviourism on the one hand, and interpretivism and constructionism on the other" (Schrøder, 2013, p. 41), the former established in the field of social sciences, the latter method preferred in the humanities. This conceptual binary has been challenged however, both paradigmatically and theoretically. Audience reception scholars facing tensions from dualist, and often duelling, positivist and interpretivist traditions that approach the audience as either passive or active, to be 
studied quantitatively or qualitatively, from an administrative or critical position, advocate for a more ecologically informed paradigm that deploys mixed quantitative and qualitative methods (Gunter, 2000; Livingstone, 2019; Morley, 2006; Schrøder, 2013). Audience research compartmentalized in discrete, autonomous categories fails to consider the complexities and contradictions of the multiple ways individuals and groups of individuals find and make meaning with media texts. Mixed methods scholars must acknowledge that all research, both quantitative and qualitative, must confront researcher assumptions and preconceptions, and that all empirical research floats in a "sea of interpretation" filtered through historically specific social and political situations (Schrøder et al., 2003, p. 52).

The theoretical foundations of audience research can be organized broadly into two factions: administrative research traditions and critical research traditions. Developed in mid $20^{\text {th }}$ century America, administrative research is, as the name suggests, conducted in the interests of administrations and funded by corporate and policy interests. Administrative researchers adhere to quantitative methods, utilizing scientific empiricism to indicate trends, patterns and generalizations of television viewing habits (Gitlin, 1978). Administrative audience researchers gathered (and continue to gather in ratings) positivist data about television audience size and composition to be analyzed and utilized by broadcasters and advertisers to make programming decisions.

Critical theory developed from the Frankfurt School to understand how mass media works in society and to query organizational structures in order to expose hegemonic power in the culture industry, "a complex of organizations that transform creativity into commodities" (Katz \& Katz, 2016, p. 5). Whereas administrative research was largely conducted to benefit corporations and institutions, "critical theory was a kind of conscience of society, to champion 
unpopular causes, and to oppose powerful establishments forces" (Rogers, 1994, p. 112). Critical scholars focused on how ordinary people were exploited by powerful institutions in society and how mass media was utilized to perpetuate their sublimation. Methodologically, early critical theorists employed mainly qualitative methods including textual analysis, content analysis and literary criticism; audience activity and their interpretation of texts was mainly assumed, "being supposedly predictable from theories of ideology and hegemony and thus neglecting concrete and local contexts" (Livingstone, 1994, p. 247).

Media effects research which developed in the years between the two World Wars was conducted to explore the ways that mass media could influence audience thought and behaviour, particularly the vulnerability audiences might have to propaganda. Much of this research was conducted in the United States using positivist methodology. To this day, quantitative and experimental methods are utilized to measure the effects of media and mediatization on audience and users (Ruddock, 2011). During the post-war years, effects scholars began to expand their theoretical and methodological efforts to explore audiences' increasing interaction with the growing number of media texts available. Lazarsfeld and Dinerman (1949) for example conducted quantitative research in the form of surveys to learn about audiences' radio listening habits while others from psychological and social psychological backgrounds (Herzog, 1944; Warner \& Henry, 1948) utilized qualitative methods to explore how listeners found meanings in radio daytime serials. Researchers began to understand that audience and text interactions were more complex than media effects research had considered. By the 1950s, these scholars shifted their analysis from the dangerous powers of persuasion of the mass media to an understanding of a more active, more discerning audience who used media for different social reasons in their everyday lives. These uses and gratifications audience researchers, much like the administrative 
audience work happening at the same time in the United States, continued to rely on positivist methods including surveys and statistical analyses, to explore the psychology of audiences and how they used media to satisfy those psychological needs (Ruddock, 2011).

By the early 1970s, scholars at the Birmingham Centre for Cultural Studies (BCCS) began to voice criticism of both media effects and uses and gratifications research for focusing on audience behaviour rather than understanding how audiences make meaning out of media texts. Hall's (1980) critiques were theoretical in terms of audience activity and the complexity of their engagement with media texts. Methodologically, cultural studies scholars argued that positivist research elevated scientific methods in order to bestow an "abstract authority" (Schrøder, 2019, p. 42) over "all other modes of experience and analysis" (Williams, 1974, p. 121). Positivist findings are both dubious and shallow and generalize audience practices without taking into account the "situational embeddedness" (Ang, 1991, p. 160) of their experiences. Cultural studies scholars are interested in audience reception rather than statistics. In other words, how “audiences differentially read and make sense of messages which have been transmitted, and act on those meanings, within the context of the rest of their situation and experience" (Morley, 1980, p. 79). Audience reception scholars presume an active audience, able to interact, interpret and extrapolate multiple meanings from a television text, often while performing other tasks (Gunter and Svennevig, 1987) requiring an acknowledgement of viewing as a "contextualized activity" (Morley, 1992, p. 165). These researchers find methodological inspiration in the ethnographic practices of anthropology, "to investigate television viewing (and the rules of its 'accomplishment') in its 'natural' setting" (Morley, 1992, p. 178).

The qualitative methods utilized for ethnographic research "in the field" include participant observation, interviews and focus groups to produce data that "attempt[s] to 
describe - and inevitably interpret — the practices of the subjects in that cultural context, on the basis of his or her first-hand observation of day-to-day activities" (Morley, 1992, p. 187). The data, in the form of notes, transcripts and observations, requires an interpretative and extrapolated analysis of an audience's interpretation of a television text. Audience reception research is in effect, an interpretation of an interpretation. This reliance on researcher interpretation creates an epistemological and ontological hierarchy that puts the researcher in a relative position of power to articulate audience meaning. Removed and elevated from subjects, the researcher risks cultural imperialism, as though the subjects are exotic others (Said, 2003) and their expressions a foreign language requiring translation. The researcher as interpreter finds and expresses and possibly imposes a meaning that the subjects themselves were unaware (Young, 1999).

Researchers such as Fiske $(1985,1987,1990)$ and Jenkins (1992), emphasize audience autonomy and empowerment to construct meaning from a television text far beyond and even removed from its encoded messaging. This romanticized, rebellious viewer, the resistant reader, engaged in "semiological guerrilla warfare" (Eco, 1990) with popular television texts was methodologically challenged for its narrow scope and failing to investigate the multiplicity of audience engagement in so-called serious texts and an over-reliance on non-representative sample sizes to gather non-verifiable and non-generalizable data (Kellner, 2011; Morley, 1992, 2006; Sholle, 1991). Morley (2006) argues that critiques of this overly active audience and resistant reading research (Nightingale, 1993), including his own (Morley, 1992), have cast a pall on audience ethnographies in general despite the persistent relevance of Fiske's understanding of television texts as polysemic. Morley (2006) warns the alternative is a reductive return to the linear model of sender-message-receiver that enlivens: 
the politics of "false consciousness," where the realm of the popular media is simply conceived as a world of "bread and circuses" got up by the powerful to dupe the vulnerable masses - though of course, it is presumed to only affect those Others (children, women, the poor, the working class, the uneducated) outside the realms of adult maturity and transcendent consciousness happily inhabited by the critic Himself. (Morley, 2006, p. 103)

Rather than supplanting ethnographic audience reception research with an alternative paradigm, Morley (2006) repositions the debate as a question of methodology. In some cases, he argues, “only statistics will help" (Morley, 2006, p. 106).

\section{A Case For Mixed Methods Audience Research}

Attempts to redress the opposition between constructivism and positivism, as polar opposites on an ontological, epistemological and methodological spectrum (Guba \& Lincoln, 2005; Höijer, 2008), have led audience scholars to seek out common ground through the utilization of mixed-methods (Napoli, 2011; Press 2006; Schrøder 2000, 2003). At its most basic, mixed methodology provides the researcher with an adaptable framework to use both quantitative and qualitative methods - surveys and interviews for one example, questionnaires and focus groups for another - in a way the researcher feels is best-suited to provide data that is both broad and deep, and that identifies trends while providing specific background, contexts, and reasoning (Creswell \& Plano Clark, 2011). The ambition is to "gain qualitative insights about and acknowledge the diversity of media users, while [saying more] about trends and regularities in a general, demographically representative population” (Huiberts, 2016, p. 4325). 
More than complementarity, mixed methods offers the researcher mutually beneficial opportunities to develop reasoning that is both deductive and inductive; generalized survey data is more deeply explored in interviews and focus groups while individual statement can be measured against wider audience trends (Huiberts, 2016).

Methodological pluralism must be more than mere juxtaposition. Schrøder (2013) argues for an integration, a near seamless combination of multiple methods in one research design resulting in a circular rather than a linear flow of support/justification/information between quantitative to qualitative and vice versa. Creswell and Plano Clark (2011) describe mixed methods as individual stories supporting a trend and, even more aptly for this research, like qualitative colour commentary supplementing the quantitative play by play in sporting events. Mixed methodology indicates a way of looking at the social world, not as compartmentalized into tidy packages, but embraces instead "multiple ways of seeing and hearing, multiple ways of making sense of the social world, and multiple standpoints on what is important and to be valued and cherished" (Greene, 2007, p 20). Mixed methodology does not privilege numeric over discursive evidence. Rather, it provides a platform for bringing them together (Danermark, 2002; Deacon, 2007; Schrøder et al., 2003)

I argue the philosophical openness of mixed methodology is suitable to this audience reception study. Audience activity or "audiencing" as Fiske (1992) would have it, occurs in multiple situations and settings and has broad implications, to the individual, within the family or community, and to society more generally. The implications of an inconsistent audience for women's hockey reach beyond the sport, beyond individual hockey players and viewers. More plainly, without an audience, the future of professional women's hockey is in jeopardy and this, I argue, has social, cultural and political implications. Women's hockey challenges the patriarchal 
ideology of frail femininity, interferes with the conflation of hegemonic masculine and nationalist identities, and has a transformative power to influence the "shifting terrain of contemporary gender relations" (Theberge, 1997, p. 70). The research question demands statistical information about audience behaviour, viewing choices and demographic attributes; however, the study also seeks to gain insight not just into audience engagement with a polysemic text, but also audience perception of the text and its broader social implications. The question isn't simply do you or don't you watch women play professional hockey, but must incorporate an opportunity to ask why. If the underlying inconsistency of viewing choices between Olympic and professional women's hockey is a possible "point[s] of dissonance and resistance" (Rowe, 2004, p. 8), it must be exploited with a methodology that allows for insight that is at once broad and deep. Quantitative methods may allow for a larger sample size but alone they are unlikely to reveal the depth of viewer opinions or experiences (Clough \& Nutbrown, 2012). Values and attitudes cannot necessarily be observed or accommodated in a formal questionnaire (Byrne, 2004). Qualitative methods provide an opportunity to thoroughly explore subjects' thoughts, practices, involvement and uses with media texts but the smaller sample set may have risked reinforcing perceptions that the audience for women's hockey is necessarily small, marginalized and therefore easily ignored.

\section{Sport Audience Research Methods}

Despite the significant amount of scholarly research focused on mediated sport, the television audience for sport has been rather underserved (Davis \& Duncan, 2006; Meese \& Podkalicka, 2015; Oates, 2012). The female audience for sport has received little attention 
(Crawford \& Gosling, 2004; Farrell et al., 2011; Hoeber \& Kerwin, 2013; Osborne \& Coombs, 2013; Pope, 2011; Sveinson \& Hoeber, 2015, 2016; Toffoletti, 2017; Toffoletti \& Mewett, 2012) and even less for female audiences for women's sport (Bruce, 1998; Meân, 2012; Whiteside \& Hardin, 2011). Few scholars go "beyond the limitations of content analysis, a methodological technique which has dominated studies looking at the amount and type of coverage given to women's sports in print and broadcast journalism” (Kane \& Maxwell, 2011, p. 202).

Kane and Maxwell (2011) employ audience reception research to investigate how fans of women's sport perceive sexualized images of female athletes in mainstream media. Their mixed method study gathered quantitative data from participant rankings of images of female athletes and qualitative data from focus groups. Their findings contradict the popular perception that 'sex sells' by demonstrating that participants reacted most favourably to images celebrating athletic prowess rather than physical attractiveness. In their article "Women Play Sports, But Not On TV: A Longitudinal Study of Televised News Media”, Cooky, Messner and Hextrum (2013) engage mixed-methods to study mainstream media coverage dedicated to women's sport, using qualitative content analysis to examine the type of coverage and quantitative analysis to determine the amount. Cooky, Messner and Musto (2015) followed up with “'It's Dude Time!': A Quarter Century of Excluding Women's Sports in Televised News and Highlight Shows”, a similarly structured mixed method study. Their qualitative content analysis indicated a shift in the way female athletes are represented in the media, from sexualized object to mother figure, while the quantitative results remained consistent, with a "dismally low" (p. 262) amount of media coverage dedicated to women's sport. Bruce (2004) conducted mixed method research within sport production studies using qualitative in-depth interviews with basketball announcers and a quantitative textual analysis of team naming patterns to explore the production of racist 
ideologies. Guest and Luijten (2018) studied fans of the Portland Thorns national soccer team by gathering quantitative data from surveys that was then contextualized against qualitative methods including ethnographic observation and interviews. Overall, there has been little mixed method research on female or male audience engagement with women's sport. This research works in part to redress this. I argue below that the utilization of mixed methods in this audience reception study not only yielded rich results about the audience for women's hockey but also demanded a rigour in data gathering and analysis that led me to draw richer, deeper and more unexpected conclusions than a singular method.

\section{Research Design}

Creswell and Plano Clark (2011) describe six design frameworks for mixed method research: 1) convergent parallel design specifying that qualitative and quantitative components be conducted concurrently; 2) explanatory sequential design beginning with a quantitative component followed by a qualitative component designed to explore the quantitative findings; 3 ) exploratory sequential design starting with a qualitative component and followed by quantitative methods to test or generalize qualitative findings; 4) embedded research design describing a quantitative study that includes a qualitative component or vice versa; 5) transformative design including either a sequential or concurrent timing of the components and devised so that the researcher may use either qualitative or quantitative components to inform or highlight the desired transformation; 6) multiphase design utilizing quantitative and qualitative methods both concurrently and sequentially over a period of time, the findings of one component informing the design of the next. 
I chose an explanatory sequential design for this study that would allow me to gather broad quantitative data from a large sample that could then both inform, and be enriched by, the follow up qualitative component. The sequential nature of the design also allowed me to recruit focus group participants from survey respondents, a strategy that increased the likelihood of reaching a more diverse range of participants, at least geographically. Lastly, the research faced time limitations associated with the scheduling of the Olympic women's hockey gold medal game and the Clarkson Cup final. The date of distribution of the quantitative component was timed to coincide with the increased public and media interest in women's hockey resulting from Canada securing a place in the 2018 Olympic women's hockey gold medal game. Team Canada was scheduled to play against their long-time American rivals at 23:10 the evening of February 21,2018 . The upcoming game was covered extensively in the press (Arthur, 2018) and on social media. My decision to launch the survey that morning allowed me to strategically capitalize on the increased public awareness of women's hockey and the hopeful anticipation that the Canadian national women's hockey team would win the gold medal.

In my initial plan, the qualitative component was scheduled to begin one month after the launch of the survey, and before the CWHL Clarkson Cup on March 25, 2018. I reasoned that one month would provide enough quantitative data that, after analysis, could inform the qualitative component. Scheduling focus groups before the Clarkson Cup would allow me to query participants about their intentions to watch the game; I would then follow up with individual phone calls to see if they did or did not watch. Scheduling the focus groups during that time period proved to be challenging; only one of the six focus groups met before March 25, 2018. This proved fortuitous. I discerned from Focus Group A that querying participants about their intentions to watch the Clarkson Cup was too close to a suggestion that participants should 
in fact tune in. I discovered in subsequent focus groups that it was a more fruitful line of enquiry to ask the question in hindsight, if participants had watched the professional women's final and their reasons and thought processes that led to that decision. While I still gathered much valuable data from Focus Group A, the other five groups' participants could speak more specifically to the 2018 Clarkson Cup. Timing was also important to ensure that the 2018 Olympic women's hockey was still reasonably fresh in participants' minds. This allowed me to ask specific questions about commentary, about production values, promotion and publicity for both the Olympic and Clarkson Cup games.

\section{The quantitative component: the online survey.}

Quantitative audience research methodologies are "framed by a positivist, empirical social scientific approach ... in which behavioural phenomena are quantified and measured numerically" (Gunter, 2000, p. 2-3). While quantitative audience methodologies include experiments (see Bird, 2003; Gauntlett, 2007; Wood, 2007), surveys have long been utilized by empirical audience researchers from the administrative tradition (Beville, 1988; Lazarsfeld, 1941), and uses and gratifications scholars (Katz, 1959; Ruggiero, 2000), albeit often as the second of a two-step process following up on focus groups (Lucas \& Sherry, 2004; Rubin, 2009; Rubin \& Bantz, 1987). While discounted by many qualitative audience researchers, for "being unable to address questions of "meaning", quantitative surveys "are ideally suited to the purpose of establishing broad patterns of media consumption and taste, if used in conjunction with more qualitative methods" (Gillespie, 1995, p. 52). Disadvantages to using surveys must be noted: the representation of the audience as stable rather than "inherently unstable" (Ang, 1991, p. 41) means quantitative data analysis must include critical scepticism. Survey responses can be 
influenced by individual's memory, experience, knowledge, and even "social desirability bias", a type of response bias that describes "the tendency of some respondents to report an answer in a way they deem to be more socially acceptable than would be their 'true' answer" (Callegaro, 2008, p. 826). Survey data does not allow for the expression of deep insight, attitudes and values nor does it allow for observable verbal or nonverbal behaviours. For this research, I argue that the survey allowed access to a larger number of respondents than qualitative methods alone and provided valuable data that indicates trends about viewing women's hockey. This allows for an assurance that the data based on a representative sample, and can therefore be reliably generalized to a population to be further explored in qualitative focus groups.

This survey was designed and distributed online using software from SurveyGizmo, a subscription-based internet service company in the United States (http://www.surveygizmo.com). Online survey research is a relatively recent tool used by thousands of academic, scientific, professional and amateur researchers around the world (Toepoel, 2015; Wright, 2005). Online survey platforms can be distributed widely rather than limited by geography or other issues of access; data entry is automated saving researchers time and effort as well as reducing opportunities for human error. Understanding that mixed methods pose challenges to researchers who lack training and/or experience (Morgan, 2007), the userfriendly nature of online survey platforms can work to mitigate qualitative researcher concerns around quantitative methods. For this research study, I weighed the benefits of the online survey in terms of software design, distribution and access against the disadvantages including limiting respondents to those able to access the internet and a necessary reliance on third party software for statistical data analysis. It is important to acknowledge possible privacy issues that may result from a private corporation that stores identifiable data online. With academic precedence as 
detailed above, the ease of distribution and user interface, the multiple reporting options and reasonable cost, and with research ethics approval ${ }^{14}$, I concluded the SurveyGizmo platform held the most promise.

SurveyGizmo allows the researcher to build a survey by choosing question types and applying display logic conditions so that a specific answer will initiate or bypass the next question. The survey for this research project consisted of 23 questions; six questions contained display logic conditions that added another potential six questions for a total of 29. The majority of survey questions were multiple choice; two of these questions included an "Other-write in" box where respondents could provide a personalized response instead of, or in addition to, one or more of the available answers. The survey also included four textbox questions asking respondents to write in their reasoning for why women should, or shouldn't play hockey, and why viewers should, or shouldn't watch women play hockey. ${ }^{15}$

The questions were designed to query respondents' interest in and knowledge of women's hockey during the Olympics and in the professional leagues. Respondents were asked about viewing habits, preferred platforms and their familiarity with the teams and players. Those who indicated they did not watch professional women's hockey were asked to choose from a number of possible reasons or to write-in their own explanation. All respondents were asked a multiple choice question about why they think viewers do not watch women play professional hockey. Other questions were designed to gauge the social importance respondents attributed to women's hockey and the significance of the gender of the player. The survey also included demographic questions about gender, age, location, education and racial identification. The final

\footnotetext{
${ }^{14}$ See Appendix A for Ryerson Research Ethics Board Approval.

15 Please see Appendix B for the Online Survey.
} 
question asked respondents to give permission for their answers to be included in the study; if a respondent indicated no, their answers were not included in the data. ${ }^{16}$

During the survey design, I identified the population to be studied, decided on the sample strategy and recruitment plan. The term "population" in quantitative research indicates the targeted group for study and the researcher must decide if they will draw out a sample or survey the entire population (Toepoel, 2015). I define the population for this study as the Canadian audience for Olympic women's hockey. Rather than reaching out to viewers who had no interest in, nor had ever watched women's hockey, I targeted audiences who had watched women play hockey during the 2018 Olympics to query if and why they may or may not watch women's hockey in the professional season. I limited respondents to Canadians in recognition of the role that hockey plays in cultural discourses in Canada that works to construct national and gendered identities. Given my knowledge and experience with the CWHL, I also knew that my queries would focus specifically on the Canadian professional league. Even though Toepoel (2015) advises, "a good list is a list on which every member of the population is present" (p. 55), a complete list of the population for this study was not available, nor particularly realistic to gather. The distinction here is between probability and non-probability sampling. In nonprobability sampling, there is a recognition that the group of respondents do not represent the entire population. Probability sampling, on the other hand, identifies a fixed group representing all members of a population. The difference is between the general audience for women's hockey as opposed to a finite population such as members of a local community hockey league. I utilized snowball sampling as a non-probability sampling strategy that relies on personal networks for recruitment, beginning with an initial respondent or group of respondents who are

\footnotetext{
${ }^{16}$ Please see Appendix C for the online survey consent form.
} 
then asked to refer someone else and so on. While criticized for failing to represent a generalized population, scholars argue "snowball sampling [is] a good way to select members of specifically defined, highly targeted populations" (Ritter \& Sue, 2007, p. 43).

The survey was launched on Friday February 21, 2018 at 08:55 ET. Utilizing snowball sampling, I posted a recruitment notice with a link to the online survey on social media and through my own personal and business contacts via emai $1^{17}$. The notice also included a request for respondents to distribute through their own networks. The initial distribution list of 93 recipients included athletes, academics, and sport leaders with a demonstrated interest in women's sport in general. My original notice was re-posted by many, including perhaps most effectively by Canadian Olympian Hayley Wickenheiser (C. Price, personal communication, February 21, 2018). This arguably increased the likelihood that the survey would be distributed to a larger sample set and appeal to respondents already interested in women's hockey.

\section{The qualitative component - focus groups.}

The robust data produced by the survey provided valuable insight into the audience for women's hockey by indicating broad trends and patterns of thoughts, opinions and viewing habits. While the embedded qualitative components in the survey began to add "flesh and blood" (Gillespie, 1995, p. 52), further qualitative research was required to examine the complex and sometimes contradictory ways viewers engage, or do not engage, with women's hockey. Given the time constraints of the research study as detailed above, I determined that collecting supplementary data in focus groups would be the most efficient technique to gather the opinions of many people at once rather than conducting individual interviews (Robson, 2002). The use of

\footnotetext{
${ }^{17}$ Please see Appendix D for the survey recruitment notice.
} 
the focus group as a qualitative research method dates back to World War II in media effects research conducted by military psychologists and civilian consultants (Merton, 1987; Morgan, 1996). Featherstone (2018) recounts a story of the first focus group as a dinner party gone awry, when, in 1941, Paul Lazarsfeld absconded with his dinner guest, Robert K. Merton, to test listeners of a new anti-Nazi propaganda program using quantitative technology that Lazarsfeld developed for this purpose. Merton took over the discussion that followed, querying participants about their quantitative choices. The result was a "lifelong collaboration between the two social scientists ... that significantly shaped the field of sociology. More importantly to contemporary culture, politics and everyday life, their friendship and partnership gave us the method that we now call the "focus group"' (Featherstone, 2018, $1^{\text {st }}$ chapter). In the 1950 s, focus groups became the favoured methodology for marketers and advertisers (Bartos, 1989; Morgan, 1997; Morrison, 2001) prompting critical media scholars to reject the method as too commercially oriented (Merton, 1987).

By the 1980s, media scholars interested in emerging critical and interpretive media research methods (Gunter, 2000) returned to the focus group as an efficient means to interrogate the complex ways active audiences interact with television texts (Merton, 1987; Morgan, 1997). Although arguably lacking specific protocols in design and implementation other than "ad hoc rules of thumb that have been passed down and then formalized in the how-to books as "good practice" (Lunt \& Livingstone, 1996, p. 82), the methodology generally consists of a group of individuals gathered together to discuss an issue, guided by a moderator who poses questions designed to elicit opinions and ensures the group remains focused on the issue at hand (Gunter, 2000). The location, size, composition and tone of the group is at the researcher's discretion and 
must be weighed and considered for impact on the quality of data produced (Lunt \& Livingstone, 1996).

In contrast to the linear question and answer of the in-depth interview, focus groups encourage discussion and interaction that can provide deeper insight into participants' experiences and opinions (Morgan, 1997). While individual audience members decode media texts, further meanings are negotiated through interactive discourse (Gamson, 1992; Lunt \& Livingstone, 1996; Swink, 2017). The challenge to the moderator is to ensure a balance between participants inclined to dominate conversations and more passive participants without causing discomfort or effectively shutting down the discourse (Gunter, 2000). The focus group provides group data, collective views that are shared and negotiated by individual members. The group dynamic must be considered in terms of familiarity, whether members of the group know each other or are strangers, share demographic characteristics such as age, gender, class etc. Some researchers (Gamson, 1992; Kamberelis \& Dimitriadis, 2013; Kitzinger, 1994; Warr, 2005) argue familiarity between focus group participants creates "peer group conversations" (Gamson, 1992) that are advantageous and efficient for bypassing introductions and positioning. Peer group conversations provide insight into social contexts where meanings are formed and negotiated. Morley (1980) points to a group identity emerging as shared opinions and values are recognized. As Gunter (2000) warns, the researcher must not lose sight that focus group participants are aware they are actively involved in a research exercise and so may self-censure or exaggerate thoughts and feelings in front of strangers or even family members. While critics suggest focus group data lacks representative value due to issues of small size or avenues of recruitment, others (Morrison, 2001) argue results are comparable to and consistent with survey findings. 
As mentioned above, the sequential nature of the research design also addressed issues of recruitment. After respondents concluded the survey by giving permission to use their data in the research, they were asked if they would be interested in participating in a follow-up focus group. ${ }^{18} \mathrm{~A}$ link was provided to a Google Form ${ }^{19}$ that asked respondents to supply contact information, as well as location and availability. The decision to use the survey as a focus group recruitment tool was more than mere efficiency; it was, I argue, best practice to ensure sampling from within the identified population of the audience for women's hockey. I was particularly interested in the focus group as an "interpretive community", identified as a gathering of participants who share certain traits and characteristics if not similar social positions and experiences. Radway (1984) argued that reader interaction with the text isn't merely an autonomous individualized experience and must be understood to include other operations, around desires to read certain texts and not others, prescribed modes of interpretation "and the uses to which both the interpretation and the act of constructing it are put” (p. 71). Ginés (2014) rejects the inherent homogeneity of the interpretive community, expanding the term to describe a gathering of diverse participants who have one thing in common, in this case, an interest in women's hockey. In this way, the researcher allows for the autonomy of the viewer in their interpretive experience while recognizing the interpretive community as a "source of cultural legitimation of texts" (p. 234).

Accessing a sample that is representative of a population while ensuring heterogeneity requires a vigorous recruitment process. Unlike an online survey that can be completed in 5-10 minutes in any location so long as the respondent can access the internet, focus group participation requires a commitment of time, energy and occasionally travel. Recruitment

\footnotetext{
${ }^{18}$ Please see Appendix E for focus group recruitment notice.

${ }^{19}$ Please see Appendix F for focus group follow up (1).
} 
through posters, online notices or snowball sampling can be effective; however, these limited strategies pale in comparison to the depth and width of the population I was able to access through the wide distribution of the online survey. While the survey did not simply serve as a recruitment tool, to ignore its potential recruitment opportunities was not productive.

The 97 survey respondents that signed up for follow-up focus group research were sent a link to a second Google Form (B) and asked to choose from eight proposed times and dates for in-person focus groups to be held on Ryerson Campus in downtown Toronto. ${ }^{20}$ Respondents unavailable to attend these times and dates were asked to indicate their interest in participating in an online or telephone focus group. Of the 41 individuals to respond to this form, nine were available and willing to participate in an in-person focus group. Only one of the available time slots was chosen by three participants, the minimum number I determined was needed for a viable discussion. Attempts to schedule the other six respondents on alternative days were unsuccessful. As a result, only one in-person focus group was conducted with three participants on Ryerson Campus on Tuesday March 20"th 2018 from 10:30 - 12:30, an hour more than originally scheduled.

The six respondents who were unable to attend in-person were asked to participate in an online focus group, along with the additional 28 respondents who had indicated this as their preference. These 34 people were sent a link to a third Google Form (C) and asked to choose from eight possible days and times. ${ }^{21}$ This Google Form also requested information about demographics (gender and age) and familiarity with women's hockey. The information was gathered in an attempt to ensure diversity; however, participant availability became the ultimate deciding factor. Each participant signed a consent form and gave a verbal agreement allowing

\footnotetext{
${ }^{20}$ Please see Appendix $\mathrm{G}$ for focus group follow up (2).

${ }^{21}$ Please see Appendix $\mathrm{H}$ for focus group follow up (3).
} 
their participation to be recorded, transcribed and included as data ${ }^{22}$. To protect anonymity, each participant was assigned a code associated with their focus group identifier (i.e. A-1, B-2 etc.). In accordance with the Ryerson Research Ethics Board, this coding information and the transcripts from the focus group audio recording are stored securely in an external hard-drive in a locked office. $^{23}$

As a research method, the in-person focus group poses a number of challenges in terms of scheduling participant availability, securing locations, organizing travel and risking a sample limited by geography. Conducting focus groups online, with or without a video component, has become a common alternative (Kenny, 2005; Stewart \& Williams, 2005) offering convenience and cost benefits to the researcher and participants while removing geographic barriers and encouraging heterogeneity and diverse opinions. Online focus groups can be conducted asynchronously when participants contribute individually on their own time, usually as textbased submissions; or synchronously, when participants contribute at the same time on video or audio chats or in online conference platforms (Abrams \& Gaiser, 2017; Kenny, 2005; Kite \& Phongsavan, 2017). Video conferencing and chats allow face-to-face communication; generally, participants are visible in thumbnail video windows spread in an array at the bottom of the screen. On many platforms, the video will expand to fill the screen when a participant speaks, although other focus group members remain visible in thumbnails below. While online focus groups remove geographic barriers, other limitations must be considered including access to the internet as well as knowledge of, or comfort with the technology. This became an issue for some online focus group participants as discussed below.

\footnotetext{
${ }^{22}$ Please see Appendix I for focus group consent form.

${ }^{23}$ Please see Appendix C for research ethics application and approval.
} 
In this study, I conducted synchronous online focus groups to more closely approximate a face-to-face interaction that "may reveal important and unanticipated data on the topic" (Abrams \& Gaiser, 2017, p. 439). Many online focus group software options were considered; Google Hangouts was chosen as a platform that provided relative ease of use, no cost and the option for video conferencing. As well, invitations to participants could be issued through the companion software of Google Calendar, reducing the chance of mis-scheduling. There were, however, challenges with this software. Invitations issued through Google Calendar included a link to join the video conference. Some participants without Google accounts reported being asked to signin. I established a number of Google accounts for this purpose. If a participant confronted a signin page they were able to input the information I provided and were taken to the video conference. On three occasions, participants were not able to join the video conference for unknown reasons and instead joined as audio contributors via telephone.

\section{Sampling Selection}

The online survey received a total of 918 complete responses and 73 partial responses. Given the explanatory sequential nature of the study that dictates the quantitative component precedes the qualitative (Creswell \& Plano Clark, 2011), I used a filter to impose a date restriction to limit quantitative data up to the day of the first focus group on March 20, 2018. I also used filters to remove partial responses as well data from respondents based in the United States. Given that I had identified the population to be studied as audiences of Olympic women's hockey, responses from 34 individuals who indicated they had not, or were not planning to watch the Canadian national women's hockey team play in the PyeongChang 2018 Winter Olympics 
were removed. This left a final sample size for the survey of $685(n=685)$. I note three spikes in response counts: on Day 1 (February 21, 2018) with 148 respondents; Day 2 (February 22, 2018) with 249 respondents; on Day 17 (March 9, 2018) with 70 respondents. Day 1 and day 2 spikes can be accounted for as the pre- and post- cursor to the Olympic gold medal game; the Day 17 spike may be attributable to press coverage of International Women's Day on March 8, 2018 and the launch of the Canadian Women's Hockey League (CWHL) playoff season.

As detailed above, focus group participants were recruited from survey respondents. A total of 97 people indicated their interest in participating in a follow up focus group; however, challenges with scheduling and communication reduced the number of focus group participants to $25(\mathrm{n}=25)$. A total of six focus groups were held as per Table 3 :

\begin{tabular}{|c|c|c|c|c|}
\hline \multicolumn{5}{|c|}{ Focus Group Information } \\
\hline Focus Group & $\underline{\text { Location }}$ & Date & Time (ET) & \# Of Participants \\
\hline A & Ryerson Campus & March 20, 2018 & $10: 30-12: 30$ & 3 \\
\hline B & Online & March 30,2018 & $11: 00-12: 00$ & 5 \\
\hline $\mathrm{C}$ & Online & April 10, 2018 & $18: 30-19: 30$ & 5 \\
\hline $\mathrm{D}$ & Online & April 11, 2018 & $10: 30-11: 30$ & 4 \\
\hline $\mathrm{E}$ & Online & April 12, 2018 & $17: 30-18: 30$ & 5 \\
\hline $\mathrm{F}$ & Online & April 13, 2018 & $14: 00-15: 00$ & 3 \\
\hline
\end{tabular}

Table 3 
In the five online focus groups, participants introduced themselves, their locations and described their interest in women's hockey. In terms of interaction, participants did not indicate any familiarity with each other either personally or by reputation. While the focus group was structured mainly around questions I asked followed by individual responses, there were occasions when participants would jump in to add or argue against someone else's answer. Focus Group A, the in-person group, was markedly different. In this case, the three participants all knew each other as teammates from a community hockey league. Whereas the online focus group conversation was much more moderate in tone with participants waiting for either the moderator to call on them or for another participant to finish talking, the "peer group conversation" of Focus Group A commonly erupted in loud debate with participants often speaking over each other. Transcribing the explosive exchanges of the in-person focus group was difficult; however, the interaction did allow an observation of non-verbal, affective expression. This suggests synchronous online focus groups allow participants to focus on questions and the discussion in general, and can generate responses that are reflective of a more natural thought process (Poynter, 2010). While data is also more easily transcribed, the moderate tone does not necessarily allow the researcher to observe participants' affective investment in the specific topic. Generally, the tone of all the online focus groups was respectful and there was a common perspective that women's hockey was worth supporting, even if individual participants were not committed to doing so as viewers. Focus group participants expressed complex, and often contradicting, descriptions of their interactions with and opinions on women's hockey, describing ways they engage with media texts, thoughts on production components, how the games are filmed and the players covered, the quality of game commentary, as well as more conceptual ideas about the social and political implications of women's hockey. 


\section{Mixed Method Data Analysis}

Data analysis describes the process by which data is represented and interpreted. Data analysis in mixed-method research consists of "analyzing separately the quantitative data using quantitative methods and the qualitative data using qualitative methods ... as well as techniques that 'mix' the quantitative and qualitative data and end results"' (Creswell \& Plano Clark, 2011, p. 203). Although discrete, the process of data analysis in quantitative and qualitative strands is not dissimilar and includes data preparation, exploration, analysis, representation, interpretation and validation. Below I will discuss the four stages of data analysis prescribed by an explanatory sequential design: 1) survey data (quantitative); 2) survey open text data (qualitative); 3) focus group data (qualitative); 4) integrated data analysis.

\section{Quantitative data analysis: the survey.}

In this study, quantitative data was collected in an online survey that was prepared and run through the SurveyGizmo. The nature of the online survey means that no data entry is required and so transcription errors are avoided. Non-probability survey results do not contain estimates of margins of error (Sue \& Ritter, 2012). While sampling errors occur in all survey research, snowball sampling and other non-probability sampling relies on volunteers to selfselect for participation and so lacks a reliable sample size to calculate the margin of error (Sue $\&$ Ritter, 2012; Toepoel, 2015). Survey data must be cleaned to "remove all unnecessary variables, entry errors, illogical answers (e.g. out of a possible range), respondent's identifying information, skip errors (good survey software already detects and deletes skip logic features)" 
(Toepoel, 2015, p. 176). SurveyGizmo offers a number of tools that help the researcher prepare and analyze data including a Quarantine Bad Responses tool that highlights responses that have been completed too quickly or too slowly, or indicates straight-lined or patterned response in multiple choice questions, or if all checkboxes are chosen. This can indicate respondents were either uninterested and/or motivated to complete surveys as quickly as possible. The Quarantine Bad Responses tool also indicates when open-ended questions are answered in nonsensical ways. SurveyGizmo also offers a software tool that assigns reporting values or a numeric to each multiple choice answer. This is important for serious, in-depth statistical analysis utilizing software tools such as SPSS (Statistical Program for the Social Sciences) (http:///www.spss.com) that requires multiple choice answers be ranked or weighted. For the purposes of this study, answers to multiple choice questions were not ranked and so I determined this level of analysis was unnecessary and that maintaining textual responses rather than numeric was sufficient for data analysis. Utilizing SurveyGizmo software filters, I removed the data from partial completions and imposed date and location limitations. While the size of the sample $(\mathrm{n}=685)$ contributes to the reliability, stability, and generalizability of this study's statistical inferences, its representativeness in terms of gender, income, race, geography and age is arguably skewed to a specific demographic. This will be discussed further in the following chapter.

To explore the quantitative findings, SurveyGizmo allows users to view the data by individual response or by a reporting tool. Individual response data is also downloadable as a *.csv file. For this study, I created a report with the filters as indicated above and also exported a downloadable *.csv file to Microsoft Excel. This allowed me to examine the data as overview, seeing statistical data as both numbered and in percentages. Distribution of data indicates trends that allow researchers to begin to formulate generalizations (Creswell \& Plano Clark, 2011). 
Examining the individual reporting, especially in a *.csv format allowed for a more granular interrogation, making bad data possibly missed by the Quarantine function easier to identify and to assess data reliability and validity.

Quantitative data analysis is done in consultation with the research question and in consideration of stable (demographics) and abstract (audience behaviour) variables (Creswell \& Plano Clark, 2011). In this study, research questions included:

- Who is the audience for women's hockey?

- Why do they watch Olympic women's hockey but not professional women's hockey?

- Why should people watch women play professional hockey?

- Is it important for women to play professional hockey and why?

Variables allow the researcher to analyze data to "understand two basic phenomena:

relationships and differences" (Wrench, Thomas-Maddox, Richmond, McCroskey, 2008, p. 106) and to establish relationships as positive (when an increase in one variable produces an increase in another variable), negative (when an increase in one variable causes a decrease in another) or neutral (variables are unrelated). For the purposes of this study, data was categorized by a nominal variable "identified by its qualitative characteristic" (Wrench et al., 2008, p. 111) and descriptive statistics that "describe or summarize information...used to organize and summarize information or data" (Wrench et al., 2008, p 146). SurveyGizmo provides analytical tools to identify and examine trends including Comparison Reports that display summarized data broken down by subgroups (e.g. gender, association with women's hockey) and Cross Tabulation Reports that shows relationships between two or more survey questions. For example, are respondents who identify as women more likely to watch women's hockey? Are viewers of Olympic women's hockey more likely to watch professional women's hockey? Do respondents 
who indicate women's hockey should be watched, actually watch women's hockey? Summaries of statistical results in the form of statements, tables or figures represent the data analysis.

Quantitative data must be validated at two levels: (1) validating the quality of the instruments used to conduct the research; (2) the quality of the conclusions drawn from interpreting the results so that " the scores received from participants are meaningful indicators of the construct being measured" (Creswell \& Plano Clark, 2011, p. 210). Internal validity represents the conclusions the researcher draws from the data that supports a cause and effect relationship between the variables; external validity represents the extent to which the researcher can confidently generalize findings from a sample to the larger population. As Creswell and Plano Clark (2011) explain further, "this means correct inferences can only be drawn if the investigator has used procedures such as selecting a representative sample" (p. 211). Assessing validity in this study included an evaluation of the online survey software SurveyGizmo as academic instrument, a comparison with external findings, such as audience ratings for professional women's hockey (please see following chapter), and an assessment of the vitality of the representative sample based on the breadth and scope of distribution as discussed earlier. Together, these factors determine the research is sufficiently robust.

\section{Qualitative data analysis: open text survey responses.}

As noted above, the quantitative strand of this study included embedded qualitative components. The survey included 2 multiple choice questions with an "Other-Write In" option while other questions posed as "why" or "why not" provided a textbox for written response. This qualitative data was explored, analyzed and coded using SurveyGizmo's Open Text Analysis tool that allowed me to define a series of categories or 'Text Buckets' used to group, organize 
and quantify textual answers into broader themes, to be then "grouped into even larger dimensions or perspectives, related, or compared" (Creswell \& Plano Clark, 2011, p. 208). Open text analysis in the form of coding is a qualitative methodology based in grounded theory. Grounded theory is conceptualized as emerging from the data, "from the ground up" in comparison to deductive methods that gather data to prove or disprove a theory (Wrench et al., 2008). Developed by Glaser and Strauss (1967), grounded theory as a method relies on ongoing comparative analysis: data is gathered and analyzed in symphony. The researcher relies on "reasoning about experience to entertain all conceivable theoretical explanations for the data and then proceeds to check these explanations empirically through further experience - more data collection - to pursue the most plausible theoretical explanation" (Charmaz \& Henwood, 2008, p. 241). The intention is to build empirically derived inductive theories of human behaviour and the social world.

While grounded theory typically provides a foundation for qualitative research, scholars have long considered the potential benefits of a grounded theory perspective for mixed method research (Glaser, 2008; Glaser \& Strauss, 1967; Creamer, 2018), although few have explored all that grounded theory has to offer to mixed method research design and implementation (Guetterman, Babchuk, Howell Smith, \& Stevens, 2019). In this study, I chose grounded theory to allow me to adapt my methodology so that the qualitative component could arise from initial quantitative findings. Rather than conducting research to prove a theory about audience engagement or non-engagement with women's hockey, my conclusions instead emerge from the data, particularly from the integration of quantitative and qualitative analyses. Unlike the theoretical sampling of grounded theory research, the quantitative component employed purposeful sampling from participants identified by a set of criteria prior to conducting the 
research. The qualitative component followed a theoretical sampling procedure that identifies a group of participants by a set of criteria and then, based on those findings, gathers more participants for further research. In this way, the quantitative findings provided data and access to participants for further research (Walsh et al., 2015). Another key grounded theory procedure involves constant comparative methods to develop analytic themes or categories emerging from organized and coded data.

\section{Qualitative data analysis: focus groups.}

Qualitative data analysis follows many of the same procedures as quantitative in terms of data preparation, exploration, analysis, representation and interpretation but differs in the type of data. Rather than weighted numerical results, qualitative data from focus groups in its raw form are analyzed as transcripts, text to be thematically arranged, ordered and coded (Creswell \& Plano Clark, 2011). As discussed above, audience research in the cultural studies tradition utilizes primarily ethnographic methods including participant observation, structured and semistructured interviews and focus groups, methods that "allow respondents to converse freely about their media experiences, choosing their own answers, their own language and terminology, and even their own questions" (Gunter, 2000, p. 39) to be analyzed and interpreted by the researcher through content analysis and/or thematic coding. A code is a precise key word that captures or represents the primary content of the qualitative data (Saldana, 2011). Coding describes the process the researcher undertakes to attach labels to portions of data that indicate a theme or subject, or reflects the essence of the content, "grouping evidence and labelling ideas so they can reflect increasingly broader perspectives" (Creswell \& Plano Clark, 2011, p. 208). Data is analyzed through a three-step process involving open, axial, and selective coding (Strauss \& 
Corbin, 1990). Open coding is an immersive, exploratory process "of breaking down, examining, comparing, conceptualizing, and categorizing data" (Strauss \& Corbin, 1990, p. 61) This involves reading the data (transcripts) thoroughly and repeatedly to establish an intimate familiarity and allow broader categories of information, such as codes or themes to emerge. Axial coding reconstitutes the fragmented material of open coding in new ways by identifying relationships among the open codes, seeking points of connection in categories and concepts. Selective coding is the process that establishes a core category of data that summarizes or accounts for the central research question or phenomenon around which the other categories are integrated. It is through selective coding that a researcher begins to develop theoretical constructions (Strauss \& Corbin, 1990).

As discussed above, audio recordings were made of all the focus groups. Transcripts of audio recordings were produced using a paid online transcription service (http://www.temi.com). Data preparation involved a process of cross-referencing the audio recordings with the produced transcripts, correcting errors and removing participant identifiers to maintain anonymity. Lastly the data was imported into a qualitative data analysis software program NVivo 12, (https://www.qsrinternational.com/nvivo) for coding. NVivo 12 is one of many online qualitative data analysis tools used with increasing frequency by academic, market and political researchers (Bertrand, Brown \& Ward, 1992; Creswell \& Maietta, 2002; Fielding \& Lee, 1991). Some critics caution against digital tools for qualitative analysis, warning data is easily isolated from contextualization (James, 2013) and can limit the creative imagination of the researcher by an overly efficient streamlining of the analytical process (Lawson, 2004). Others (Maher, Hadfield, Hutchings \& de Eyto, 2018) argue that the management and organization of the necessarily rich qualitative data must be of primary concern in analysis and conclude "digital software proves to 
be an invaluable tool for this process" (Maher et al., 2018, p. 5). The authors conclude that current software is insufficient on its own to "scaffold" data analysis, suggesting supplementary digital coding with more traditional means (sticky notes, coloured pens, visually mapping on a physical surface) to allow for more “'serendipitous encounters' and creative exploration of ideas and interpretations" (Maher et al., 2018, p. 12). For this research study, I found the human computer interface of NVivo 12 to be both user-friendly and efficient for data immersion, exploration and organization. While acknowledging the ongoing importance of traditional coding methods, in this study, I argue that the software more than sufficed. This is echoed by other researchers (Houghton et al., 2017) who argue NVivo offers additional advantages "to systematically and rigorously synthesize findings" (p. 873).

NVivo 12 software provides a number of tools that support data analysis including note or memo-taking software as well as tools to identify emerging themes and categories defined in NVivo 12 as nodes. Once the qualitative data was uploaded into NVivo 12, the material was read multiple times to establish familiarity while remaining "flexible to emerging insights" (Maher et al., 2018, p. 2). I began with open coding to create tentative categories from the meaning that emerged from the data. Axial coding allowed me to identify relationships or connections between the open codes as a kind of conceptual alchemy. With selective coding I first identified the core variables that applied to all the data and then reread then the transcripts to code any data that relates to the core variable. For example, utilizing open coding I identified the ways women's hockey was described as better than men's hockey. Axial coding allowed me to make connections between statements about skills, lack of violence and nostalgia and selective coding allowed me to see how these statements function to position women's hockey as "pure". See Table 4 below for the three stages of coding: 


\section{Table 4}

\section{Qualitative Coding}

$\begin{array}{lll}\text { Open Codes } & \text { Axial Codes } & \text { Selective Codes } \\ \text { Association with hockey } & \text { Demographics } & \text { Sample homogeneity } \\ \text { Spectating/not spectating } & \text { No information } & \text { Audience interest } \\ \text { No promotion } & \text { Broadcaster failing } & \text { Media coverage } \\ \text { Role models } & \text { Hegemonic femininity } & \text { "Pure" Hockey } \\ \text { Equity } & \text { Hockey for social good } & \text { "Pure" hockey } \\ \text { Hockey like it used to be } & \text { Nostalgia } & \text { "Pure" hockey } \\ \text { Skills } & \text { Better than men } & \text { "Pure" hockey } \\ \text { No physical play/violence } & \text { Better than men } & \text { Affect/cognitive hockey }\end{array}$

Table 4

Assessing the reliability of qualitative research, Guba and Lincoln (1989) determined qualitative findings must accurately ('truthfully') reflect participants' reality (credibility), be transferable to other contexts and situations (transferable), be consistent and repeatable (dependable), and are shaped by the participants and not the researcher (confirmable). In this study, multiple focus groups produced data from different participants, situations and contexts and yet from this disparate data emerged similar and consistent themes. From this, I argue, these qualitative findings meet Guba and Lincoln's reliability markers of credible, transferable, dependable and confirmable. In this study, I acknowledge a preconception or bias clearly stated as a desire for the audience for professional women's hockey to grow. Yet, the findings of the qualitative and quantitative research do not functionally support a realization of this 
preconception, bias or desire. Instead, the research findings help to build knowledge of the actual and possible women's hockey audience in Canada from which theoretical and practical discussions can emerge.

\section{Mixed Methods: A Grounded Integration}

Charmaz and Henwood (2008) argue that the integrative, comparative, emergent conceptualizations of grounded theory make it particularly well-suited to mixed method research. Grounded theory informs the integrative or mixing component of this study. Creswell and Plano Clark (2011) dictate that the steps for mixed data analysis in explanatory designs begin with independent analysis of each strand followed by a process that transforms qualitative findings into quantitative data and quantitative findings into qualitative data to allow for a side-by-side comparison of merged results to establish categories for coding. The mixed method findings are presented in a discussion which becomes "the vehicle for merging the results" (Creswell \& Plano, 2011, p. 223). In this study, comparative analysis involved qualifying quantitative data through descriptive statistics summarizing respondents' attitudes, opinions, behaviour and sociodemographic attributes in a general trend or pattern. Qualitative data was quantified both manually and through NVivo 12 to provide statistics on focus group participants' perspectives. However, rather than a more unidirectional flow where qualitative data inform quantitative data, following grounded theory, comparison was ongoing and sometimes circular. By this I mean, analysis of the quantitative strand enriched and informed the qualitative research which produced data that would then circled back to enrich and inform the quantitative findings in comparison and so on. For example, the majority of survey respondents indicated a lack of publicity as the 
reason why they didn't watch women play hockey; focus group questions based on this trend allowed that concept to be interrogated more deeply and from which emerged the finding that participants wanted not just more publicity, but to feel a deeper connection to the athletes. Survey data about the disconnect between the large majority of respondents who think women should play professional hockey and the much smaller percentage who had actually watched women play professional hockey provided statistical structure to the qualitative findings from focus group participants who articulated a dedication to women's hockey with a failure to watch. In this study, quantitative findings enriched and support qualitative findings as much as the qualitative data functioned as explanation of survey results.

There are many advantages to mixed methods research, particularly in audience studies where the divide between administrative and critical research has appeared impassable. There are limitations to mixed methods in general, however, and to its utilization in this study. Mixed methods research requires extensive effort, time and resources and requires a broader range of skills (Creswell \& Plano Clark, 2011). With the breadth and scale of the information gathered, researchers risk overload leading to a shallow rather than deep analysis (Huiberts, 2016). There is an inherent complexity in designing, analyzing and interpreting mixed methods research that requires reflexivity and care to consolidate divergent traditions and epistemologies. Researchers may be reluctant to undertake a mixed method study for lack of rigorous training in one method or the other, an outcome of "the real world of university research institutions [when] one often encounters research environments that are still in the grip of myopic methodological straitjackets, in which the issue of methods is not a matter of choice but of prescription" (Schrøder, 2013, p. 328). Support for designing the quantitative strand was found in literature, through the SurveyGizmo website, academic support and from external experts. A limitation of 
this mixed method research was in its distribution and sampling. While snowball sampling is an academically acceptable form of recruitment, a targeted sample consisting of a defined group recruited through a broadcaster or even women's or girl's hockey associations may have allowed for a more verifiable representative sample than the non-probability survey conducted. Given the time limitations, relying on my own network was the most efficient and practical means to draw a reasonably vibrant sample. I understand that research methods, like research itself, gains momentum through familiarity and practice.

Another limitation to the mixed methodology employed in this research concerns the measurement and articulation of audience's affective engagement with women's hockey or the affective circulation between text and viewer. In other words, the study asked subjects to articulate how they feel about women's hockey and how women's hockey makes them feel. As discussed in the previous and following chapters, affect theory provides the foundation for an understanding of how affect flows in, through and between audience and the television text. Anna Gibbs (2011) makes the connection between affect and feeling and the formation of beliefs that:

depends on the embodied aspect of affect, on its power to make the heart beat faster, or the muscles to tense, or the skin to grow cold and clammy or to burn with a blush. The corporeal origin of affect and its power to transform the physiological state of the body in response to ideas is what makes belief real, both sustaining conviction and lending it urgency. (p. 256)

The visceral effect of affect helps to affirm and make real the cognitive processing. In this study, I relied on respondents and participants to cognitively articulate or describe their affective state; the data consists of a cognitive articulation of previous affective state rather than data of the 
visceral effect of affect. Gorton (2009) identifies a similar issue in her original research question about audience affective engagement with television texts. She describes how this had to be put aside in the face of the difficulties of setting up a situation where participants would be able to feel or communicate their feelings to her in her presence: "part of the experience involves the sense that one is alone or in an intimate surrounding. Having an interviewer at someone's side, recording and observing, breaks this feeling of intimacy" (p. 144). Taking affect back to its corporeal nature, where affect flows in and through the body, future audience research may incorporate wearable technology measuring biometrics as affective response to media and media texts. ${ }^{24}$ Researchers then don't have to rely solely on participant language to express and translate (if even possible without editorializing) affective circulation. Too, this approach allows for the accumulation of quantitative data that answers many of the critiques aimed at qualitative audience research as being prone to generalizations and prioritizing the text. Despite these limitations, mixed method in this study yielded rich results that provide opportunities to develop theories for why audience watch, or won't watch, women's hockey.

\footnotetext{
${ }^{24}$ See Stead, Gouley, Evans, Mamdani, 2011 work in "Emotional Wardrobe", clothing outfitted with sensors indicating changes in the emotional states of the wearer.
} 


\section{Chapter 4 Findings}

The intention of mixed methodologies is to merge theory and practice to disrupt and problematize traditionally narrow research procedures that fail to take into account the multiplicity and multilayered nature of marginalized experiences (Hesse-Biber, 2010). This mixed method audience study utilized both quantitative methods for larger sample sizes with more heterogeneity, and qualitative methods that provide more context, background and nuanced data, albeit within a more limited sample group (Hankivsky \& Grace, 2015). The explanatory sequential design of this mixed method research stipulates that quantitative data can be explored, contextualized and enriched by qualitative data. Results from the survey allowed me to hone the lines of inquiry I would pursue in the focus groups. Additionally, statements from the focus groups provided deeper understanding of survey data.

As detailed in the previous chapter, I began this mixed methods research with a quantitative survey from a population I defined as Canadian individuals who had watched or planned to watch the Canadian national women's hockey team play in the 2018 Olympic Winter Games in PyeongChang. The qualitative component took the form of focus groups with participants recruited from survey respondents. While the data gathering was sequential, I am presenting the findings below, mixed as it were, under headings that indicate categories or codes that emerged from the data as follows: (a) Audience Interest; (b) Media Coverage; (c) "Pure" Hockey; (d) Affective Engagement; (e) Sample Homogeneity. I argue that the integration of the findings here allows for clear connections to be established between the data sets and creates a foundation for the results to be discussed in the next chapter. 


\section{Audience Interest}

As mentioned in the previous chapter, the population to be studied was identified as audiences of Olympic women's hockey. To ensure the integrity of the sample, a filter was imposed to remove data from survey respondents who indicated they had not, nor were planning to, watch the Canadian national women's hockey team play in the 2018 Olympics. No surprise then that the overwhelming majority respondents indicated that women's hockey was either very important $(69.9 \%)$ or somewhat important $(25.7 \%)$ to them among all the winter Olympic sport (see Figure 1). More interesting was the lack preference to watching Olympic women's hockey over the men's. Just under half (46.7\%) indicated they would prefer to watch women's hockey over the men's; slightly more (47.2\%) said they would watch both equally and $6 \%$ claimed they would prefer to watch men's hockey (see Figure 2).

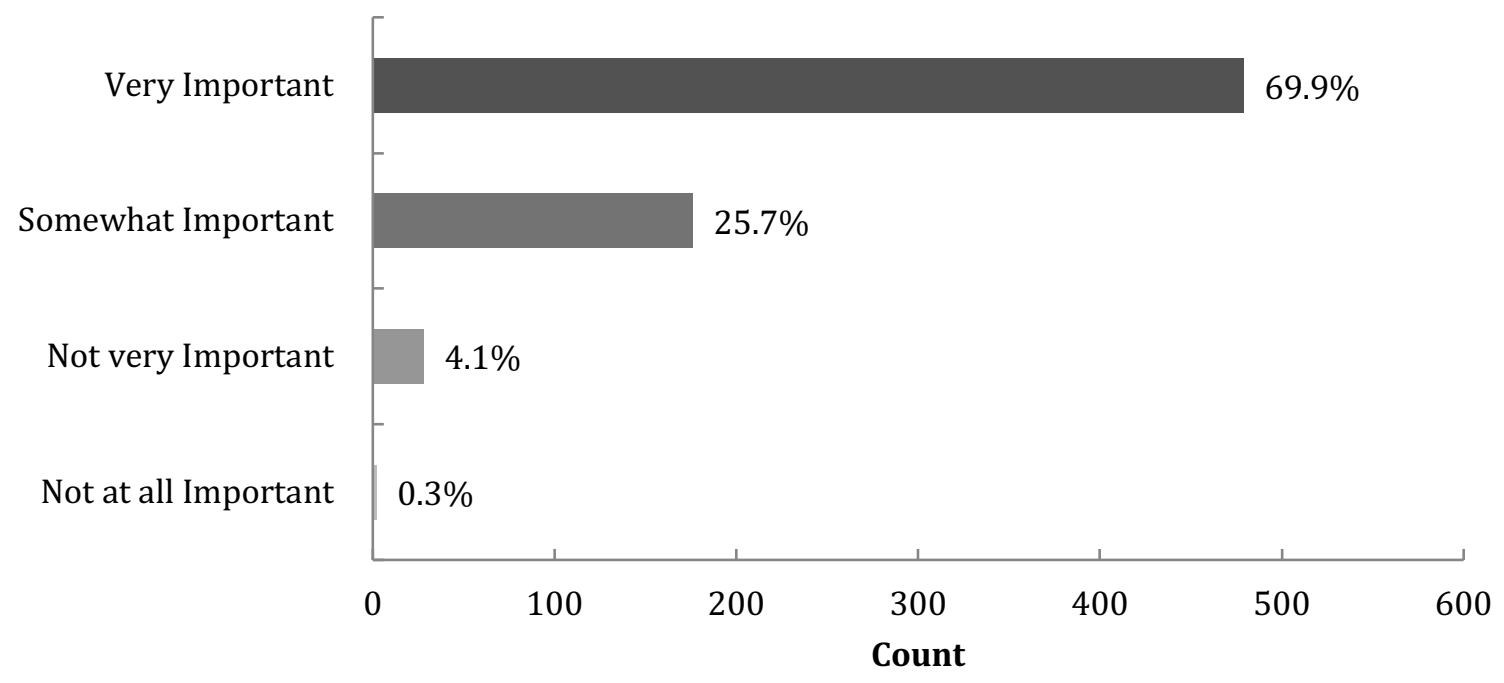

Figure 1. Q1) Among all winter Olympic sports, how important is hockey to you? 


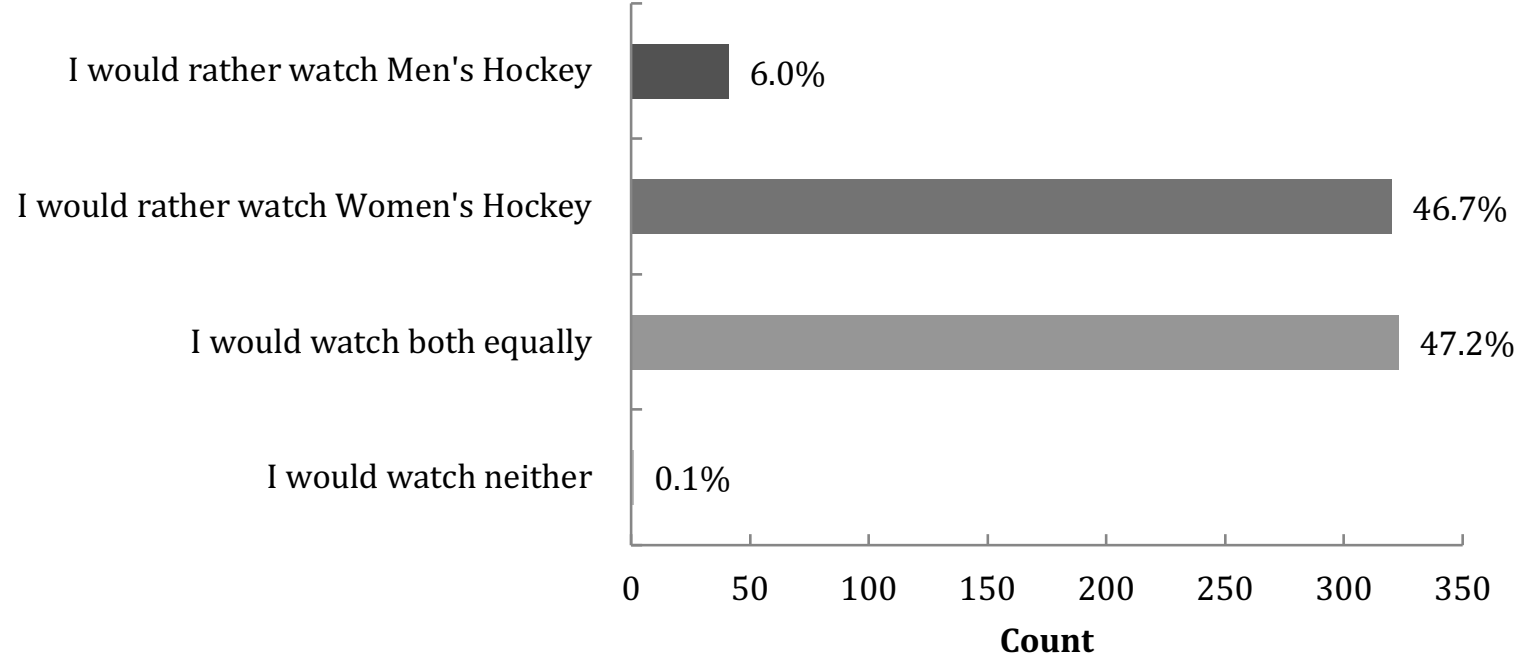

Figure 2. In the Winter Olympics, would you rather watch Men's Hockey or Women's Hockey? That the majority of respondents indicated a lack of preference could be attributable to the broadcast scheduling during the Winter Games and the rare occurrences when men's and women's hockey occupy overlapping broadcast slots. During the Pyeongchang Games, for example, there was only one instance on February 15, 2018 where the broadcast of a men's hockey game overlapped with a women's hockey game (Pyeongchang 2018: TSN National, 2018). In other words, viewers rarely have to choose between men's and women's hockey. Only $6.0 \%$ of survey respondents indicated they would prefer to watch men's Olympic hockey. The lack of preference for men's hockey could be attributable to the gendered majority of respondents or to the Canadian national men's hockey team's failure to make the gold medal round. Certainly, there was a disparity in ratings (see Table 5). Men's hockey, usually the ratings winner of the Winter Olympics (Clipperton, 2019), ranked well below the women in 2018. Low audience interest in Olympic men's hockey may have resulted from the lack of National Hockey League (NHL) stars playing on national teams. After 20 years of allowing its players to compete in the Olympics, the NHL rescinded the policy for PyeongChang. Reporters note this as the 
reason the Canadian men's team failed to make the gold medal rounds and for the overall low profile of men's hockey at the 2018 Olympics (Hutchins, 2017a; Longley, 2018a).

\section{Table 5}

Canadian Television (CBC) ratings for PyeongChang 2018 Olympic Winter Games

Sport: Event $\quad$ Ratings

Figure Skating: Virtue/Moir Ice dance gold medal free skate 5.7 million*

Hockey: Canadian women's gold medal game 4.8 million*

Figure Skating: Weaver/Poje ice dance short program 4.1 million*

Figure Skating: Team figure skating gold-medal awards $\quad 4.1$ million*

Snowboard: Parrot/McMorris slope style medal win $\quad 3.7$ million*

Snowboard: Sébastien Toutant's gold medal win in big air. $\quad 3.7$ million*25

Hockey: Bronze medal game, Canada vs. Czech Republic $\quad 2.8$ million $^{26}$

Table 5

While it can be argued that the failure to make the finals may have benefited the women's team in terms of audience ratings, the majority (76\%) of survey respondents indicated the lack of NHL players made no difference in their decision to watch women's hockey. This disregard was also expressed in the focus groups; their decision to watch Olympic women's hockey was based on a number of factors as discussed below, but the lack of NHL players on the men's team was not one of them. However, $21.6 \%$ of survey respondents indicated the NHL lockout left them more

\footnotetext{
$25 *$ Rody-Mantha (2018)

${ }^{26}$ Numeris TV Meter, Feb. 24, 2018, CBC Total + TSN++ SN + SN1, A2+, Sat 7a-10a, Total Canada, AMA, generated by InfoSys $+\mathrm{TV}$
} 
inclined to watch the women. I argue that the data from the survey and the 2018 Olympic ratings puts the stability of audience for men's hockey in question (Phillips, 2018). As mentioned in Chapter 2, broadcasters seeking to expand the audience base for men's hockey incorporated "female-friendly" content that would also not alienate the male viewer. This paints a picture of a stable men's hockey audience, unwilling to accept alternative forms of (e.g. women's) hockey. The survey results clearly shows a more porous hockey audience, willing to engage with multiple hockey texts.

Interest in women's hockey was often explained through association with the game, as a player themselves, or as a family member of someone who plays hockey. Survey results indicate $35.6 \%$ of respondents played hockey and $73.3 \%$ either played and/or were associated with hockey through a family member. Of the $35.6 \%$ respondents who played hockey, $78.6 \%$ selfidentified as female/CIS gendered female. In focus groups, 21 out of 25 participants indicated an association with hockey as a player and/or through immediate family: "I can bet you that if I was not exposed to it as a kid watching my sister play, I probably wouldn't seek it out” (E-4).

I have to say that I have had no interest in hockey in hockey really until my daughter decided to take up hockey as an alternative to figure skating. She has become this like diehard hockey kid, so I would love to be dialed into women's hockey more and I think it would be terrific for her to have the opportunity to watch more women's hockey. (C-4) Association for some participants involved driving daughters to and from arenas and volunteering in recreational hockey leagues:

I've been the registrar for our leagues for the last eight years. So I'm very involved in girl's hockey; in fact tryouts started last night and I was at the rink from four to a midnight last night, so I'll be there again tonight from four to midnight. (D-3) 
Others coached daughters' teams or participated in fund-raising activities. In some cases, it was the daughters who instigated or inspired the family to watch broadcasts of women's hockey: "my daughters both adore it. They play at the rep level and uh, you know, any opportunity I get, I like for them to be able to watch hockey on TV. That is representative of their gender" (C-1).

Some focus group participants who played hockey articulated their interest to watch women's hockey as a way to gain information "I would watch for the need to build up a knowledge of the team and the players" (A-1); "I watch games differently now try to understand what they're doing. I'm watching you know, what their systems are and watching it separately" (B-1).

For me it's this idea of the craft of hockey. So I would watch a game to learn what Szabados, how the fuck does she make those double pad sings? How can I do that and who on my team can I pass the puck to get that" (A-2)

Many focus group participants identified their association with women's hockey as the foundation for an affective engagement with the media text. This connection was articulated as both emotional: "I'm just a player and I just love watching women play hockey" (B-5), and physiological: "It's almost like I can relate way better to the women and I feel motivated and driven and like that could be me out there" (B-2), "I guess because I'm a woman, I love watching women do sport, um, you know, live vicariously through them" (D-1), "when you're watching a hockey game, do you feel in your body? I do" (A-2). I will discuss this in greater detail and will argue in the next chapter that affective engagement is a key component when strategizing audience building.

The question of whether interest in and/or knowledge of professional women's hockey translates to broadcast viewing was queried in the online survey. While $89.9 \%$ of respondents 
were aware of professional women's hockey (see Figure 3) and 75.0\% expressed an interest in watching women's hockey (see Figure 4), just over half (55.0\%) of respondents indicated they had ever watched a professional women's hockey game (see Figure 5).

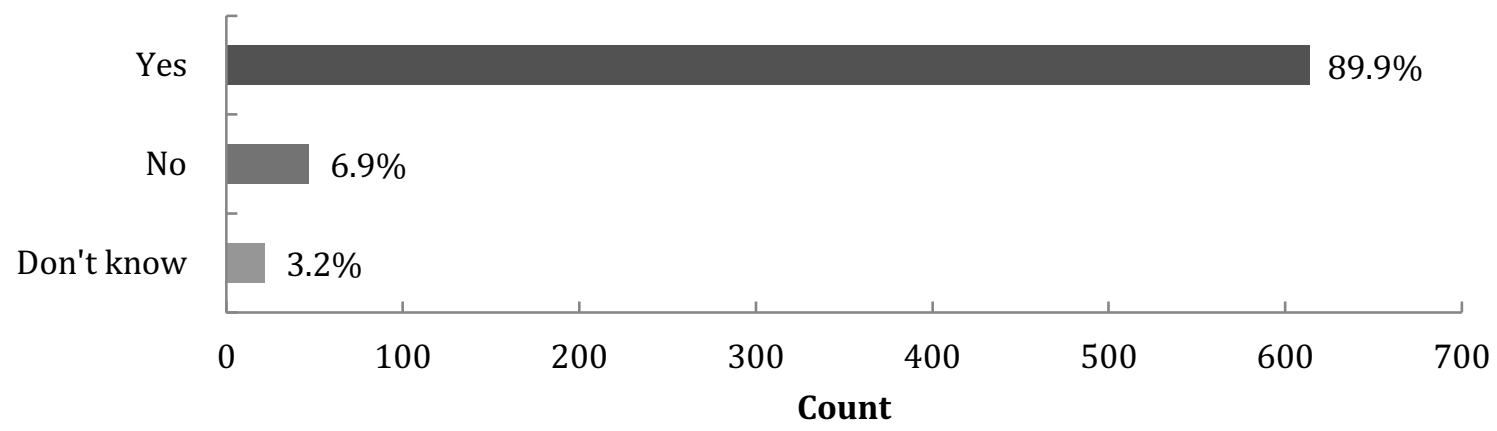

Figure 3. Q9) Are you aware that women also play professional hockey?

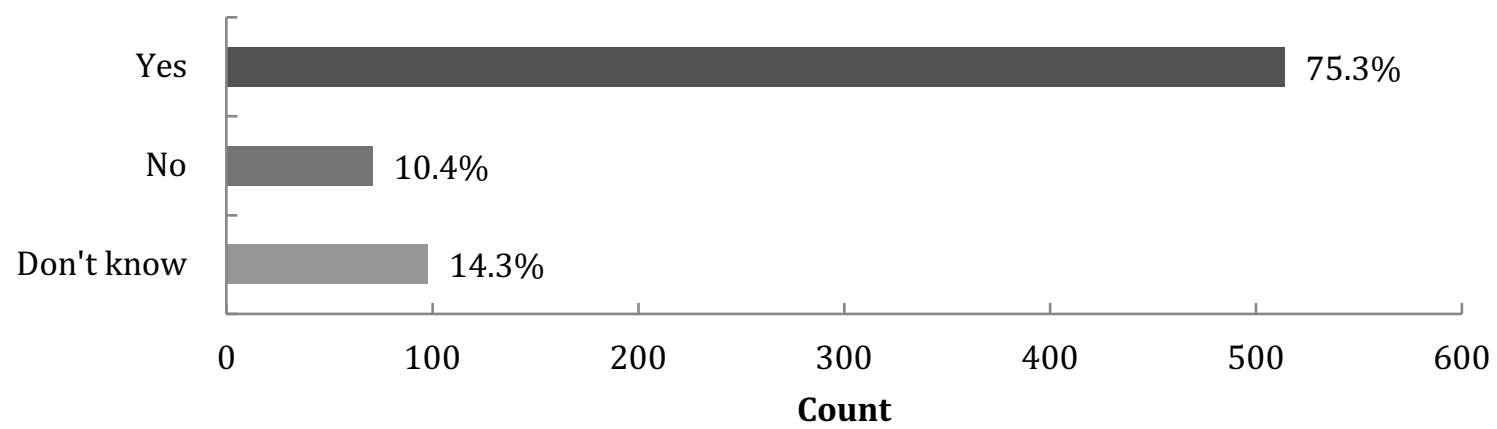

Figure 4. Q13) Are you interested in watching women play professional hockey?

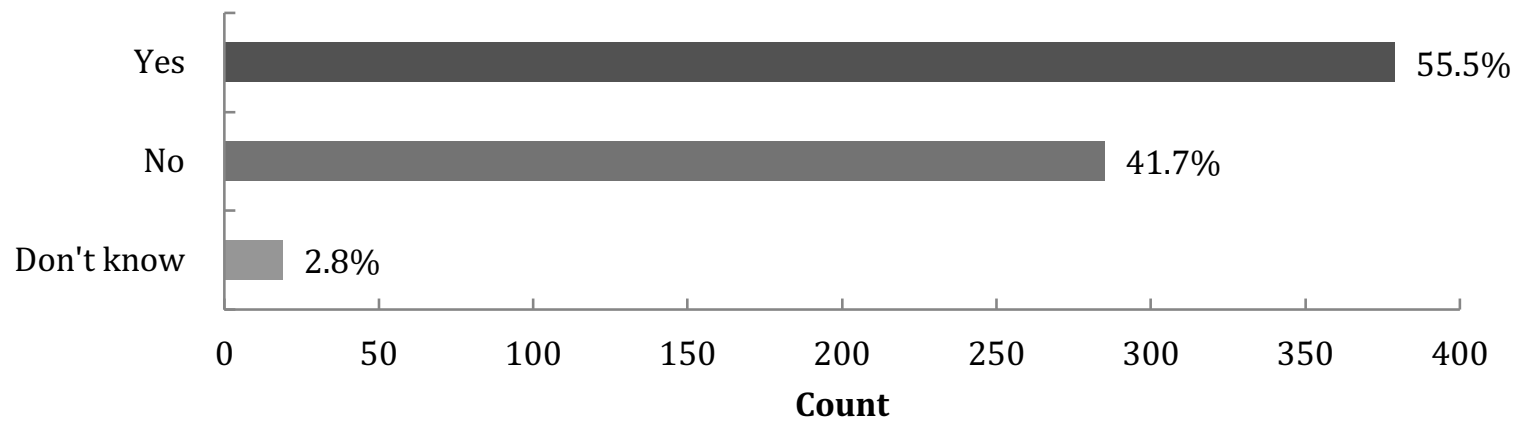

Figure 5. Q11) Have you ever watched professional women's hockey? 
When asked if women should play professional hockey, $98.0 \%$ of survey respondents said yes and $100 \%$ of focus group participants agreed. This data supports the hypothesis of the research question: there is interest women's hockey, particularly during the Olympics, and a commitment to the general belief that women should play hockey, but that does not translate to viewing habits for the professional women's leagues. However, here we can add that interest in women's hockey does extend beyond the Olympic Games to the professional leagues. The majority of respondents enthusiastically supported the idea of professional women's hockey and expressed a desire to watch both broadcast and arena games and yet $42.0 \%$ of survey respondents had never watched a professional women's game.

Interest in and awareness of professional women's hockey was expressed both consistently and keenly in the focus groups. Perhaps not surprising given they were willing to commit the time and energy to participating, all focus group participants articulated their interest in and desire to support professional women's hockey. And yet, more than half (52\%) had never watched a game in either professional league. Of the $48 \%$ who had watched a CWHL game, few (8\%) claimed to be consistent and dedicated viewers. Instead, these participants explained they would watch if there happened to be a game on, if they had run across a broadcast rather than seeking it out. "I do watch the occasional game but I'm not like consistently watching men's or women's hockey. My biggest preference in terms of watching is watching in person which I mostly get through coaching" (C-2). "I'm one of those people who don't really watch other than when the Olympics are on or if I catch, I'm going around the channels I find women's talking and then I'll watch" (F-2). 


\section{Media Coverage}

When online survey respondents were asked why they haven't watched professional women's hockey, more than half (59.4\%) chose “I don't know where I can watch professional women's hockey", while $42.0 \%$ answered with “I don't know when I can watch professional women's hockey". 17.8\% weren't aware that professional women's hockey existed (see Figure 6).

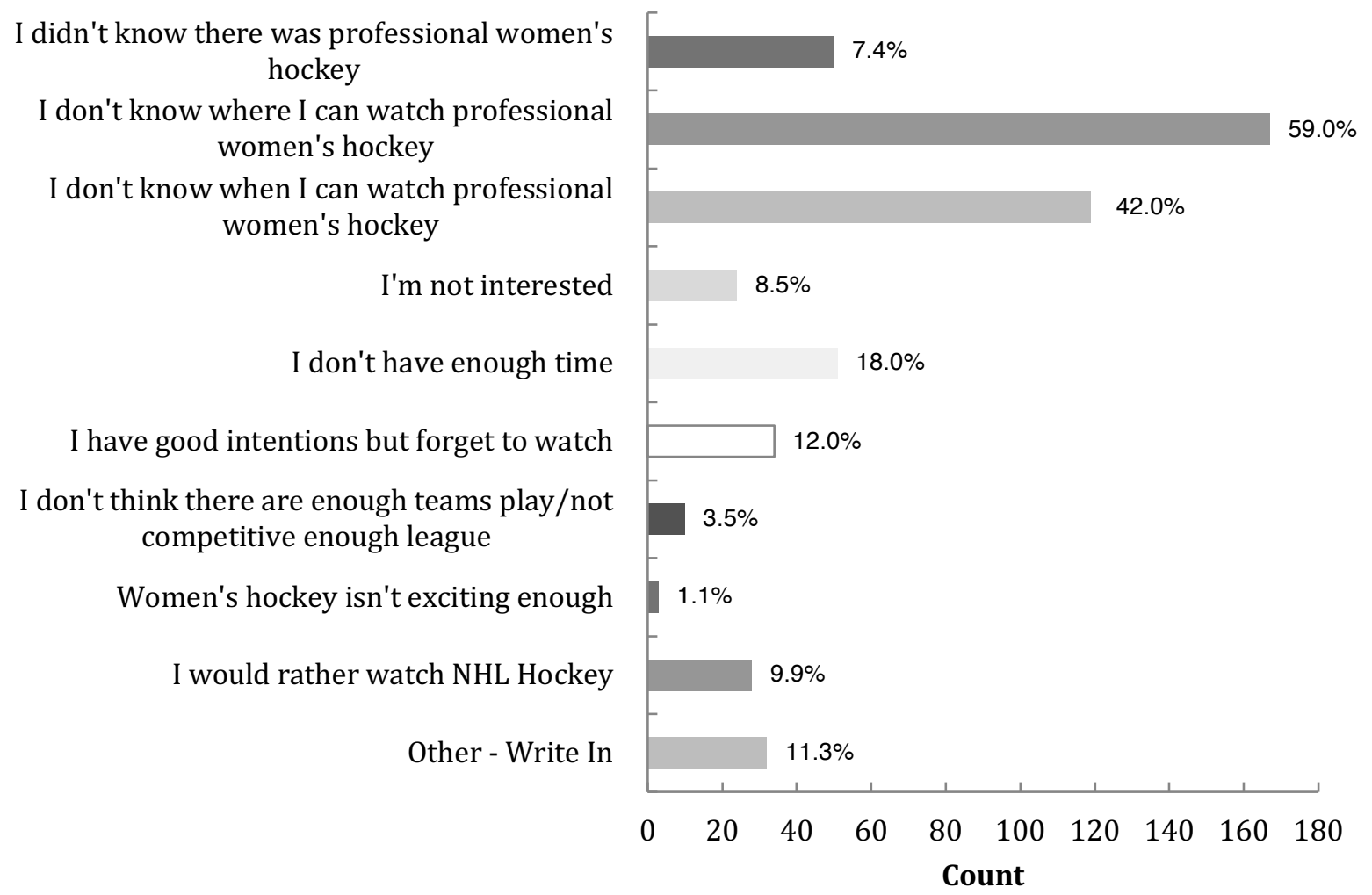

Figure 6. Q12) Why haven't you watched professional women's hockey?

In answer to the question of what might compel them to watch, $71.6 \%$ of respondents indicated they would need to know where and when professional women's hockey was broadcast; this was further supported by the $51.9 \%$ who said they would watch if there was more advertising and 
promotion about the games. Fewer respondents thought knowing more about the teams (30.1) and the players $(22.5 \%)$ would convince them to watch (see Figure 7).

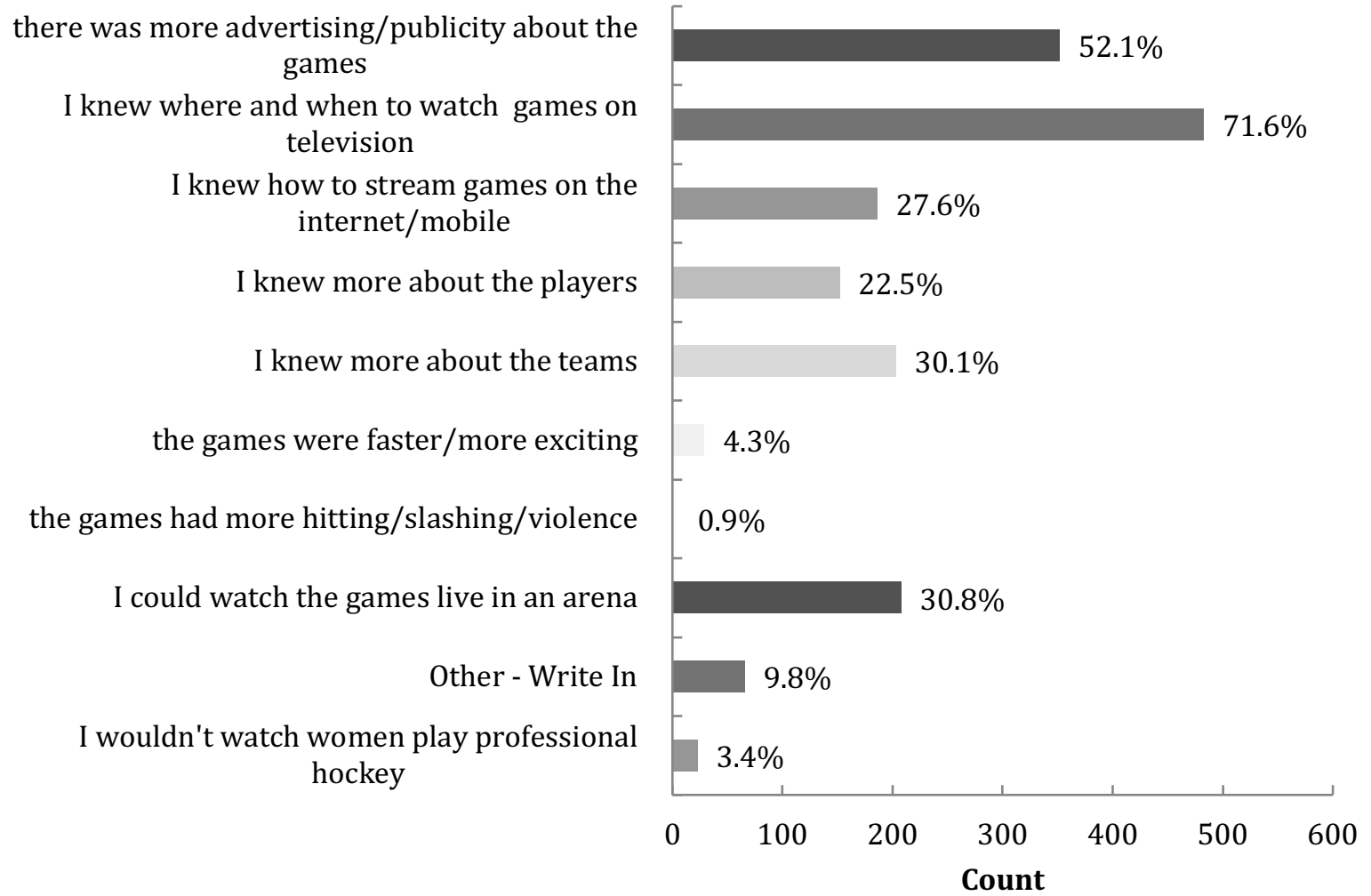

Figure 7. Q14) You would watch professional women's hockey if...

These results were also reflected in the focus groups. Participants explain that not knowing when or where professional women's hockey is broadcast was the overwhelming reason used to explain their inability to watch. Many pointed specifically to the lack of marketing and publicity from both the broadcaster (Sportsnet) and/or the league (CWHL):

I think the advertising is a big issue. We don't always know when the Games are, where they are. If it's on TV, definitely. I will put it in my bookmark and say, hey, I need to watch the game. I think it's information that is lacking. It's the media coverage in the news or whatever, but we don't talk much about women unless it's something big (D-2). 
Brenda Andress, the commissioner of the CWHL, answered this critique with empathy but some frustration explaining the marketing strategy with the broadcaster Sportsnet:

For two weeks before our game, it's all over Sportsnet, like it's all over it which is unbelievable...they put a ton of money into it, we put a ton of money into it. I mean our website has the games. All they have to do is go on the website and if they go on the website they'll be able to see where the games are. We send out marketing materials to every single women's group that plays hockey and ask them to distribute it. I hear their frustration. Could we do a better job of marketing? Probably. But that would mean more money ... to have the marketing dollars, you still need the fans you can only do so much without the fans being engaged. (personal correspondence, June 14, 2018)

Sportsnet does promote CWHL broadcasts across TV, Digital and Social platforms that “integrate great stories and interview into our production" (Amanda Dwyer, personal correspondence, October 16, 2018). However, the campaign does not include the Clarkson Cup which is promoted exclusively through the CWHL. It remains that for viewers who do not watch Sportsnet or check on the CWHL website, awareness of the league and their games is low. This becomes an issue in terms of audience growth and the difficulty reaching potential viewers who know little about professional women's hockey: "I think we are preaching to the choir. I suspect, you know, the marketing and promotion goes to the Facebook group, the Instagram followers, the people who are already engaged and likely to watch" (E-3). Focus group participants noted promotional efforts by the CWHL, including free game tickets to local girls' hockey leagues, but the translation of this to larger broadcast audiences was seen to be ineffective.

Even when there was some awareness that CWHL games were broadcast, focus group participants blamed the scheduled time as a deterrent. The 2018 CWHL season broadcast on 
Sportsnet consisted of 3 games as follows (times are all ET): 13:00 Sunday January 28, 13:00 Saturday February 3, and 17:00 Saturday March 10 (Nafio, 2018). Participants referred to the lack of a consistent broadcast schedule negating the opportunity to create habitual viewing as well as the inconvenience of the primarily early afternoon time slot interfering with other commitments:

I have to go get groceries and I have to do my cooking for the week that I'm getting together with friends later, so I have to get all this done before this time and then, you know, sitting down and watching a game on a Sunday afternoon falls down the priority list. Whereas if I was getting together with my friends later on, we could watch the women's game if it was at a different time. (B-3)

Other participants noted the afternoon broadcast slot often conflicted with their own hockey playing time. Participants were definitive in their beliefs that primetime broadcast was securely owned by men's hockey for the male market and there is no incentive to share the value of that time and place: "We get male hockey. Every game is covered. Are all the women's games covered? No, of course. And why? Because anybody will tell you TV money is worth millions of dollars, and that's a male market" (A-3).

Participants also noted that the afternoon broadcast time lessened the opportunity to watch CWHL hockey with friends in public spaces such as a sports bar where viewing becomes more of a shared, social event, particularly compared to watching men's hockey when “there's just this uproar of energy and culture surrounding your favourite teams or playoff season. That culture just doesn't really exist for women's hockey" (B-2). Intentionally developing an audience culture around women's hockey was perceived to have not only financial value in terms of 
audience building, but also social repercussions as a statement of legitimacy for women's hockey in particular and women's sport more generally.

Discussions in the focus group included the amount of promotion and marketing efforts and the type of coverage sports journalists give to professional women's hockey. Participants observed that mainstream sports news neglect stories about specific games, athletes and stats in favour of broader or meta-stories such as whether CWHL players will get paid or whether the two professional women's leagues should merge to survive:

Like when Hilary Knight joined the Canadiennes; I think that that's the kind of story that would drive fan engagement. When they get bounced out in the semi-finals. That's the kind of sports stories that are engaging at a fan level but aren't getting communicated widely enough that the large media outlets don't have people covering women's hockey. (B-1)

The inclusion of women's hockey in daily sports reporting on television and radio news would serve to "normalize" women's hockey, to integrate it into a broader hockey culture and expand the market. Stories should be less focused on the issues and problems with women's hockey and more about the players and game play. Participants articulated value for this in a number of ways: increased audience provides more financial structure for the league to expand, more opportunity to pay players and negate the need for a second job. In turn, this leads to better quality of play and more competitive teams and ultimately provides a possible career goal for the young women and girl hockey players of today.

For focus group participants, media coverage also means game coverage, both in terms of the quality of commentary and who is calling the play-by-play. Some were explicit in their opinions about male commentators providing questionable coverage of the women's game and 
more prone to use gender inappropriate language such as "defensemen" rather than defensive player. Others argued that the women's game needed better coverage and that the gender of the commentator was irrelevant. The issue for these participants is that male commentators are better because they have the experience; the problem is the lack of opportunity for women hockey players to graduate into visible media roles, noting that the few who do are often treated harshly by fans and other journalists:

I think that they're working on getting the female journalists out there. You've got Christine Simpson now covering men's hockey, Cassie Campbell's been covering the games in Calgary, getting ripped for it mind you. But I think that she's working towards that. You've got a Cheryl Pounder. But, I agree. I think that it doesn't really matter if it's a man or if it's a woman, you just need somebody who knows what they're doing in there. but I do think that we actually do need, you know, some kind of support from the NHL to make it happen, to make it legitimate, which is ridiculous because it is absolutely legitimate. (B-4)

The issue of borrowed equity from the men's game was more thoroughly explored when participants were asked about the appearance of controversial Hockey Night in Canada host Don Cherry during the 2018 Olympic gold medal final. Publicly, Cherry positions himself as a strong supporter of women's hockey even while he has built his brand on being:

openly hostile to those who want to diminish expressions of hegemonic masculinity, including fighting, within the game...Those players who live up to his ideas of masculinity —almost always "good ol' Canadian boys"-are the centre point of his hockey broadcasts. (Allain, 2008, p. 474) 
The 2018 Olympic women's hockey games on CBC featured Mark Lee as play-by-play announcer, Cassie Campbell-Pascall as colour commentator, and Rob Pizzo and Cheryl Pounder as reporters (CBC Unveils Star-Studded, 2018). Between the first and second, and second and third periods of the gold medal game, Don Cherry appeared with Cassie Campbell in a special edition of Coach's Corner, to give his thoughts on the women's game and players. Focus group participants had strong reactions to his appearance: "He's one of the reasons I don't watch men's hockey" (D-1); "He didn't really talk about the women's game...He kept talking about the men's game, didn't really know who the players were .. it was an embarrassment to see him speaking about women's hockey" (B-4); “I can't stand the man, I don't think a lot of women can, but at the same time it does it, I hate to say it, but he lends a bit of credibility" (A-1). Other participants saw Cherry's appearance more pragmatically:

at the same time when you hear Don Cherry, what do you think? You think hockey! So I think there could have been an attempt to draw in more viewers or to get more people interested in the women's game because if Don Cherry is there that might draw a new audience or a bigger audience in, even though we may not love him, people do associate him with hockey. (B-2)

The majority of focus group participants viewed Cherry's appearance as a distraction. Instead, they valued commentators from former players such as Cassie Campbell and Tessa Bonhomme, as much for their expertise as for their value as role models, an example aspiring female sports journalists and retiring players to follow. Participants also attributed a value to female hockey commentators, particularly former players, as a way to validate women's hockey, particularly for the men's hockey audience. As B-1 explains: 
It was the World Juniors Hockey Championship and Tessa Bonhomme was working for TSN. She was interviewing players between periods. She interviewed a junior player. After, Bonhomme was doing a wrap up as the camera followed the player. We see him walk down the aisle to another player and gesture as though to say: did you see who that was? That really famous player just interviewed me. And I thought it was really cool that this world junior boy player was just totally stoked that he was interviewed by THE Tessa Bonhomme. And I thought this is important.

In this case, the adult women Olympic gold medal winning hockey player and decorated reporter gains credibility when recognized by a Junior men's player. That this story is told by a selfdeclared women's hockey fan indicates the instability of the women's hockey audience is a much deeper issue than media coverage and commentary.

\section{"Pure" Women's Hockey}

The online survey included two multiple-choice questions meant to gauge respondents' belief in the idea of women playing professional hockey. The question "do you think women should play professional hockey" was answered in the affirmative by an overwhelming majority of respondents $(97.7 \%)$ while a smaller percentage $(81.9 \%)$ answered yes to the question "do you think more people should watch professional women's hockey". Respondents were then asked to explain their choices by answering open-ended questions that asked why women should or shouldn't play professional hockey, and why more people should or shouldn't watch professional women's hockey. After analysis, I determined that the questions of why women shouldn't play professional hockey and why more people shouldn't watch women play professional hockey 
produced little valuable data. Most respondents took issue with the question itself: "I can’t answer for others", "people should watch whatever they want" and "they should if they want to". On the other hand, the data asking respondents to explain why women should play professional hockey and why more people should watch women play professional hockey held more promise. An open-text analysis of the answers to the question of why women should play professional hockey identified connections and themes from four codes emerged: 1) Gender equity; 2) Quality of game; 3) Better than men's; 4) Role models. While statements were clearly associated with one code, others required the assignment of more than one category (see Table 6).

\begin{tabular}{|c|c|c|c|c|}
\hline ample Coding: Q19) Why should women play professi & nal hockt & & & \\
\hline Open Text & $\frac{\text { Gender }}{\text { equity }}$ & $\begin{array}{l}\text { Quality } \\
\text { of game }\end{array}$ & 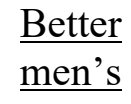 & $\begin{array}{l}\text { Role } \\
\text { models }\end{array}$ \\
\hline $\begin{array}{l}\text { Because they are as good and exciting as men but play } \\
\text { a cleaner and more strategic game. }\end{array}$ & & $\mathrm{X}$ & $\mathrm{X}$ & \\
\hline $\begin{array}{l}\text { women in professional hockey create examples for } \\
\text { younger women. Seeing women modelled in pro sports } \\
\text { is a good part of having younger women and girls } \\
\text { choose these sports as activities. }\end{array}$ & & & & $\mathrm{X}$ \\
\hline $\begin{array}{l}\text { Why not? It's fun, they are good and they can make } \\
\text { money doing it. }\end{array}$ & & $\mathrm{X}$ & & \\
\hline $\begin{array}{l}\text { They are just as skilled as men, and deserve the } \\
\text { paycheck. }\end{array}$ & $\mathrm{X}$ & $\mathrm{X}$ & & \\
\hline why the hell shouldn't they & $\mathrm{X}$ & & & \\
\hline $\begin{array}{l}\text { Great game, great role models for girls, We're } \\
\text { Canadian! }\end{array}$ & & $\mathrm{X}$ & & $\mathrm{X}$ \\
\hline
\end{tabular}

Table 6 
The quantitative results showed three quarters $(77.8 \%)$ of survey respondents felt that women should play professional women's hockey in answers coded as gender equity. Fewer (26.6\%) identified the quality of the women's game as the reason why women should play professional hockey. Some (6.2\%) articulated that the women's game was not only as good as the men's but better. Other respondents (5.2\%) reasoned that professional women's hockey players act as good role models for young women and girls (see Table 7):

\begin{tabular}{|c|c|c|}
\hline Code & $\underline{\text { Percent }}$ & $\underline{\text { Count }}$ \\
\hline Equity & $77.8 \%$ & 462 \\
\hline Quality of game & $26.4 \%$ & 158 \\
\hline Better than the men & $6.2 \%$ & 37 \\
\hline Role model & $5.2 \%$ & 31 \\
\hline
\end{tabular}

Table 7

Open text analysis of the answers to why more people should watch professional women's hockey resulted in the same coding. In this case, the results for the first two codes were reversed. The majority (76.2\%) indicated quality of game: "it's an exciting high-skilled game with incredible athletes", "it's a fine sport with excellent games and players", "it's a great game". Far fewer (26.8\%) referred to reasons associated with equity: "to support the next generation of female athletes", “to help promote women's sports", "support the women's sports". Some respondents (14.2\%) argued that the women's game was not only good enough to watch but actually better than the men's game "it is exciting and without violence", "it is a better game", "so they can see that women have just as much skill as men and aren't as big as babies as men 
are when they get hurt". Similar to Q19 responses, 4.9\% referred to the value of women hockey players as role models (see Table 8).

\begin{tabular}{|lcc|}
\hline \multicolumn{2}{|l}{ Table 8} & \\
Q21: Why should more people & watch professional women's hockey? \\
\hline Code & $\underline{\text { Count }}$ \\
Quality of play & $76.2 \%$ & 353 \\
Equity & $26.8 \%$ & 124 \\
Better than the men & $14.2 \%$ & 65 \\
Role Model & $4.9 \%$ & 23
\end{tabular}

Table 8

I began to note that many respondents who referred to the quality of the game often articulated this as a comparative or alternative to the men's game. Respondents described women's hockey as "less violent", "more skill based", "good, clean hockey" and "a purer sport". Some respondents reasoned that people should watch because the players were "in it for the love of the game" and "not as corrupt (yet) as the men's pro". In these statements, women's hockey becomes associated with social good, an answer to (men's) hockey violence, commercialism, star system as well as an antidote to gender inequity and to provide role models for young women and girls. From this emerged an additional code, "Social value". The findings show that over half of respondents $(55.5 \%)$ reasoned that watching women's hockey has a social value (see Table 9). Of the 353 respondents who referred to the quality of the women's game as the reason why more people should watch women's hockey, 159 articulated this by referencing the men's game and stating or implying that the women's game was not just better than men's hockey but better for hockey in general. 
Table 9

Q21: Why should more people watch professional women's hockey?

$\underline{\text { Code }} \underline{\text { Percent }} \underline{\text { Count }}$

Quality of play $\quad 76.2 \%$

Social value $\quad 55.5 \% \quad 266$

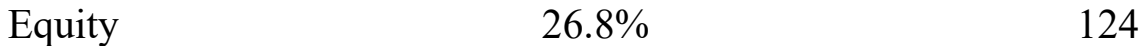

Better than the men $\quad 14.2 \% \quad 65$

$\begin{array}{lll}\text { Role Model } & 4.9 \% & 23\end{array}$

Table 9

Answers to the question of whether or not women should play hockey are more dramatic: of the 667 people who agreed that women should play hockey, $535(80.2 \%)$ articulated their reasons by referencing its social value (see Table 10).

Table 10

Q19: Why should women play professional hockey?

\begin{tabular}{lll|}
\hline Code & Percent & Count \\
Social value & $80.2 \%$ & 535 \\
Equity & $77.8 \%$ & 462 \\
Quality of game & $26.4 \%$ & 158 \\
Better than the men & $6.2 \%$ & 37 \\
Role Model & $5.2 \%$ & 31 \\
\hline
\end{tabular}

Table 10

In focus groups, the association between women's hockey and social value was articulated by many group participants, particularly in the value of female hockey players as role models: "they serve as excellent role models for young women" (B-2); "I just love the idea of a national sport love the idea of girls being involved and having the role models to, you know, pursue their dreams" (E-1); "the role modeling is critically important; there is a phrase, you can't 
be what you can't see" (E-3). The female hockey player as role model also acts as a safety net to keep young women hockey players from straying too far off the beaten path of gender expression:

I think the role-modelling and the exposure is very, very important. But I think one message that is getting out there that is positive, is that young fit, strong women do not work in any way against femininity. Right? It certainly was an attitude. Being fit and strong is something to be celebrated more than it was 20 or 30 years ago, which I hope makes it easier for young women girls to pursue their dreams in sport. (F-3)

For E-4, role modeling has specific political and social implications "I think that role models are an important part of gender equality; if society would be motivated by equality, then we should be having equal funding media companies that are advertising for both types of hockey". For some focus group participants, women's hockey functioned as a “microcosm for what we're looking at in society now" (E-5), where systemic issues of gender inequity were player played out:

I think it's great to see this sort of societal shift and I think things are getting better and they're getting more equal and yet we still have these, these areas where there's this, legacy effect, these insults, these judgments, the, you know, sports are a man's thing and, and that sort of thing. And I'm glad to see that it's changing. But I do think that it's a pretty slow change. (C-5)

The implication is that women playing hockey is good for women and girls and good for society. Others argued that women's hockey was also good for the game of hockey. The narrative logic often began by describing the women's game in contrast to the men's, as better, more reliant on skills and passing and speed of the puck than "smash them up kind of thing to watch" (C-1). 
"There's not the hitting, there's not the physical violence, it's more like a European style game where its finesse" (B-5). "It is more sportsman-like and there's more team play and there's little to no fighting" (A-1). "I do have more respect for the women in the game because I think they play smarter. They seem to me more intelligent. They don't seem to duke it out like the men in the NHL do" (E-1).

Not to say that the women's game isn't physical, it's certainly, certainly is, but it becomes more of a positional game. It's about passing lanes, it's about systems and it's speed of the puck and it, it's really, as I say, it's what the, what hockey should be like. It's the pure nature of the game. (B-1)

For others, the qualifier isn't better but different, "the same sport but different game" (C-2). In some cases, this comparative also became a statement of temporality, a nostalgia-tinged wistfulness that perceives the women's game as what the men's game used to be:

There's a lot of talk about the original six and there's so much romance around that and this idea that authentic hockey is the original six. When you look at that, it's like, well yeah, they played like we do, it was, it was more of a team ethic and I don't like the word efficacy, but in my mind like that, that was, it makes for a nicer viewing. (A-2) Based on passing, skill, intelligence and purity, women's hockey becomes, not just as good as the men's game, but its possible saviour, mired as it is in controversy around issues of fighting and the value of star players over the team (Goldberg, Sandikci \& Litvack, 2018). As I will argue in the following chapter, the same characteristics used to elevate women's hockey also reaffirm gender hierarchy and hegemonic femininity.

While a common theme in all the focus groups, the association between women's hockey and hegemonic feminism did occasionally cause some tension. One participant, referring to the 
camaraderie of her teammates, described hockey "as the new knitting" (A-3). This caused a disturbance in the group with another participant pushing back against the perception that knitting and hockey are marginalized as women's hobbies.

I have recently taken up knitting. I have a feminist textile art practice. Hockey is not the new knitting. Fine craft, or any sort of craft, has been seen as women's work and has been demeaned its entire life. I don't want hockey to be with the new knitting. I'm saying 90 percent of the people when we walk outside that door, if you said that to them, they'd be like, oh, crafty and cute. (A-2)

While focus group participants routinely valued women's hockey with characteristics associated with performative femininity, here at its most blatant it instead becomes a struggle to wrest hockey away from essentializing discourses that reinforce hegemonic gendered constructions.

\section{Affective Engagement}

As discussed in Chapter 2, sport media relies on a circulation of affect between the text, viewer and screen. As a result, it was important to query survey respondents and focus group participants about both their thoughts and their affective engagement with, or feelings from, watching women's hockey. A multiple-choice question provided survey respondents with a number of ways to describe Olympic women's hockey. The most popular choices were “exciting” (88.8\%), “fast-paced" (75.6\%) and "skill-based" (73.4\%). Just over a third (36.8\%) felt that women's hockey as good as the men's while $28.5 \%$ thought it was better (see Figure 8 ). Many who wrote in answers described the women's game as “different” from the men's, others felt women's hockey "needed to grow" and some were "inspired". When asked to choose how 
they felt while watching the Olympic women's hockey team play, $81.3 \%$ of survey respondents reported feeling "proud" and 78.0\% chose "excited" (see Figure 9). Some respondents (10.8\%) wrote additional descriptors such as "inspired", "patriotic", “interested", "nervous", "impressed" and "empowered" while $1.8 \%$ of respondents indicated they felt nothing.

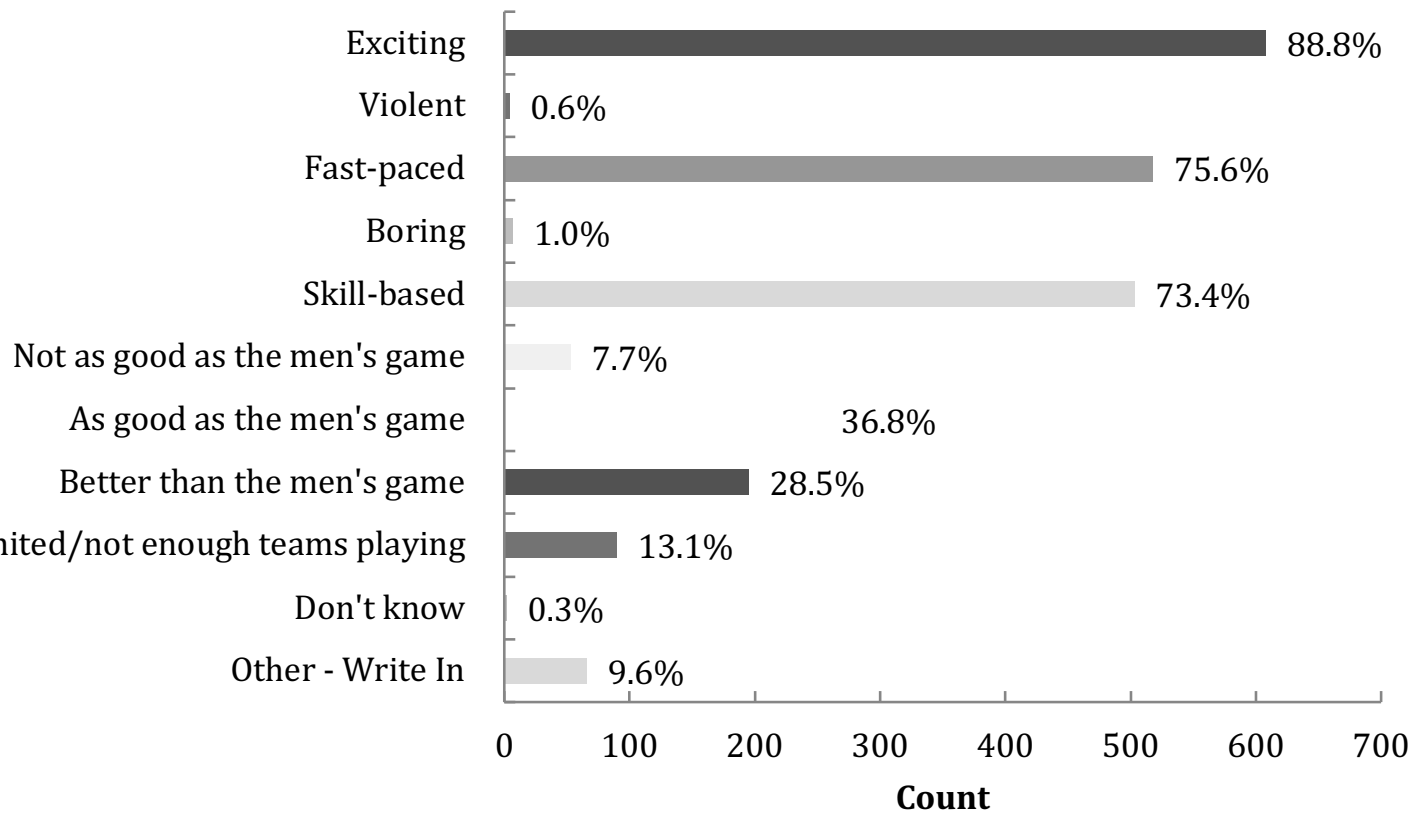

Figure 8. Q7) Do you think Olympic Women's hockey is...

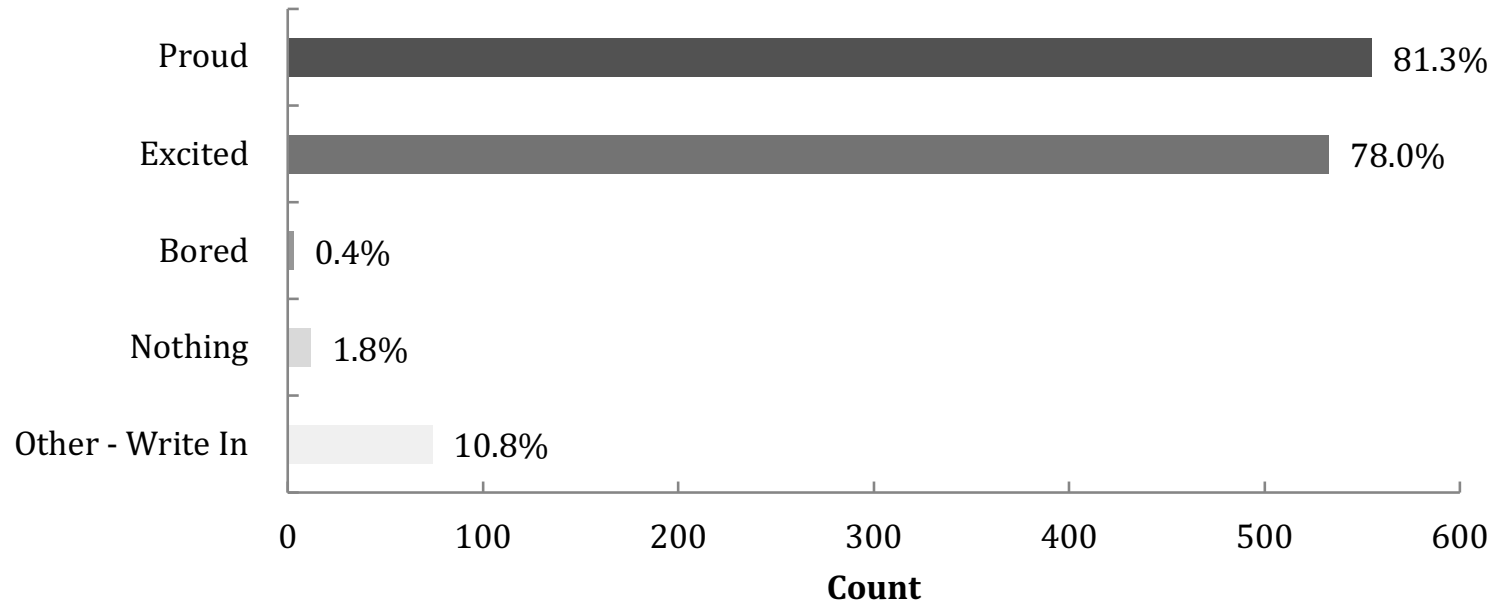

Figure 9. Q6) Watching Canadians play hockey during the Olympics makes me feel ... 
Affective engagement was articulated more precisely in the focus groups. Participants described the pride they felt while watching Olympic women's hockey, specifically a nationalistic pride deepened by the sense that this was a feeling shared with the rest of the country. This finding is expected given that participants were self-identified as Olympic women's hockey audiences. For some, watching the national women's team play made them feel excited, joyful, nervous, stressed and, during the Canadian women's gold medal loss, disappointed and sad. These feelings or the depth of feelings were contextualized by some participants as resulting from the fact that this was the women's team; the feeling of pride was based in the sense that the team's performance, even existence, was evidence of Canada's commitment to gender equity. As D-1 described:

I just get so emotionally involved because you're seeing women do something that's always been such a male dominated environment and maybe that's it. Maybe that's why it's so near and dear to our hearts.

As focus group participants discussed their emotional engagement with women's hockey, the topic of conversation veered between Olympic women's hockey, professional women's hockey, live hockey games and sometimes, actually playing hockey. The participants that played hockey articulated their watching practices in a variety of specific ways. Some described a cognitive practice watching women play hockey as a means to gather information about skills and play or to "build up knowledge of the team and the players" (A-1), "to see how the plays were crafted ... and try to mimic them. How can I do that and who on my team can I pass the puck to like that?" (A-2). Others described the feeling of watching women's hockey with an embodied empathy: "You watch them on the ice and you can almost imagine yourself doing that, 
right? Like maybe it's subconscious or maybe it's not" (F-1). B-2 articulated this particularly in contrast to how she watches with men's hockey:

when I watch women's hockey I feel energy. It's almost like I can relate way better to the women and I feel motivated and driven and like that could be me out there. But when I'm watching the men's hockey, there's just the fans and the excitement and the fight. Like more passion comes up for me when I watch women play hockey.

This idea was developed further by A-2 who described how while watching the game, she would feel as though the action of the game itself was within her body:

it's like I learned the term proprioception. Knowing where your body is in space, it's an experience I have when I'm making art and when I'm playing hockey. When you're watching a hockey game, do you feel it in your body? I do.

For some focus group participants, emotional investment with the game came from their association with it, as players, but also through family members:

I was exposed to women's hockey growing up and that's why I have a personal connection to the sport ... I can bet you that if I was not exposed to it as a kid watching my sister play, I probably wouldn't seek it out. (E-4).

Most characterized an emotional engagement resulting from knowledge of the players, the teams and the stakes: "Just to watch two teams that I'm not really a fan of wouldn't be as exciting for me. So certainly there has to be that rivalry and that kind of connection to be emotional" (E-2). However, some participants described their emotional investment watching women's hockey without having any knowledge or context of the game, exemplified by this comment: "for me hockey is hockey and I enjoy watching it” (B-5). 
In many instances, participants relate this back to the lack of media coverage of the sport and particularly the players, their personalities and backstories:

if you have a personal connection with the player and you know about their history and how they got there. You know their story. Brigette Lacquette? She was the first aboriginal person to play on the national team. There's a connection and if you're an aboriginal girl, now you're watching her with a different eye, this is a different kind of player now to you and she's more meaningful to you then what she does on the ice. But if you don't know that story then you lose the opportunity to have that kind of emotional connection. And that's one of the big advantages that men have, that there's more engagement because we know all the stories. (B-1)

Participants called for more media coverage of professional women hockey players that focus on personal stories, their backgrounds, how they came to play hockey, their hopes and dreams for the future. In the survey, only $7.2 \%$ could name all the players on the Canadian women's national team, $69.2 \%$ of respondents indicated they could name some of the players and $13.9 \%$ couldn't name any. $77.2 \%$ had heard of the CWHL, $48.2 \%$ knew about the NWHL and $18.4 \%$ hadn't heard of either league. Focus group participants identified the lack of in-depth of knowledge of teams, players and league as a barrier to watching and following the sport. Participants called for richer coverage of the sport of women's hockey, specifically around the mythic storied games from the past. And, as with most mythologies, these are moving stories filled with heroes. For example, two participants in different focus groups told the same story about the 2007 International Ice Hockey Federation (I.I.H.F) World Women's Championship held in Winnipeg. Canada was dominating a woefully mismatched Germany. 
The German goalkeeper stood on her head for about a period and a half. Canada snuck a couple by her, but it was three to one and then we scored the fourth goal and the floodgates opened, as happens in sport. But it was in a sold out Winnipeg arena when they ended up subbing out the German goalie. I only found out afterwards she was 16 or 17 year old. I mean she was a young girl. The crowd gave her a standing ovation. Then she won player of the game. They gave her another standing ovation. (F-3) And the standing applause must have been 10 minutes long. I'm not trying to exaggerate at all. It went on and on and on because the audience that appreciated the game, appreciated the players and appreciated the sports-personship of the game? And that for me was one of those moments in sport that is just a real highlight. And it isn't connected at all with the win or loss. It's connected with the way that the women can play. And it apparently it got to Angela Merkel in Germany, she got it in a text right away, and it was on the national news in Germany. And you know, Canadians responded in kind to the skill and tenacity of the German players. (D-4)

Again here, as discussed earlier, women's hockey is seen to be a vehicle for social good. In these stories, the sentiment is based less on a comparison to the men's game than it relies on a more traditional gender coding of femininity: compassionate and caring. In this case, the feelings are emitted from a presumably gender-mixed crowd embraced as they were in the warmth of the women's game.

\section{Sample Homogeneity}


A demographic profile of survey respondents emerged from the quantitative data in terms of gender, cultural identification, location and personal association with hockey. The majority of respondents $(77.8 \%)$ identified as female while the minority $(19.3 \%)$ identified as male. There was a smaller representation outside the gender binary with $1.5 \%$ identifying as gender variant/non-conforming, $0.4 \%$ transgender male and $0.1 \%$ as transgender female. $1.6 \%$ preferred not to say. The small representation from non CIS-gender respondents is notably contrary to the gender inclusive nature of women's hockey as vigorously argued by some focus group participants and as evidenced by the CWHL's well-publicized acceptance of transgender player Jessica Platt on the Toronto Furies, and with Harrison Browne of the NWHL. The lack of transgender participation in this study arguably indicates more a failure in distribution than a lack of interest from these groups. Women's hockey as a site of gender inclusivity is an important consideration and will be discussed in the next chapter.

Results indicating respondents' level of education was similarly skewed with over half (58.7\%) claiming some post-secondary education (39.2\% Bachelors and 19.5\% Masters). The age of respondents was more balanced with over a quarter (26.9\%) 45-54 year olds and just under a quarter (24.2\%) 35-44 year olds, 16.0\% 55-64 year olds and 13.4\% 25-34 years of age. Geographic region also showed imbalance with well over half (61.0\%) of respondents from Ontario; other provinces were minimally represented with just $16.0 \%$ of British Columbians making up the next largest group of respondents followed by 13.2\% from Alberta. Over half (58.2\%) of respondents identified their living situations as urban, $27.4 \%$ suburban and $14.4 \%$ from rural communities. Results indicating the cultural identification of participants shows even greater skewing with $90.0 \%$ identifying as white; $2.1 \%$ as South Asian; $1.8 \%$ as First Nation, 
Inuit or Metis; $1.6 \%$ as Chinese, $0.7 \%$ as Black, $0.6 \%$ as Arab. Other identifications had results $<0.5 \%$ while $3.2 \%$ indicated "other" and $3.2 \%$ "preferred not to say".

Of the 26 focus group participants, 17 responded to the question of gender selfidentification requested at the time of scheduling. 14 (82.4\%) self-identified as female/CISgendered female, $2(11.8 \%)$ as male/CIS-gendered male and $1(5.9 \%)$ as gender variant/nonconforming. Other quantitative demographic data in terms of cultural identification, age, education, location and community was not specifically collected from focus group participants although in many occasions some demographic information was provided in conversation. According to transcripts, most focus group participants indicated Ontario residency; two participants indicated British Columbia as home province, two from Manitoba and two from Quebec. One participant was American although, as with the survey, his data was removed.

The results indicate that the majority of survey respondents were female/CIS-gendered female, white, urban, between the ages of 35 and 54, and with some form of higher education. In focus groups, the majority of participants identified as female/CIS-gendered female. This demographic homogeneity must be interrogated as being indicative of either ineffectual survey distribution and recruitment and/or reflecting a lack of diversity in women's hockey. Snowball sampling relies on distribution through established networks; while my efforts to ensure broad distribution included recruiting high profile athletes, sport academics and leaders, community hockey groups and associations, reaching out specifically to various regional hockey associations may have helped provide a more geographically even sample set. The lack of racial and ethnic diversity in the sample set is more troubling and indicates either a failure of distribution and/or systemic racial discrimination in women's hockey. I will discuss this further in the next chapter. 
This chapter has summarized the key findings from the quantitative and qualitative components of this mixed method audience reception study. In the next chapter I will challenge the chicken-and-egg argument that limits media coverage for women's hockey by identifying an interested but unserved audience. I will discuss the discursive construction of women's hockey as 'pure' and explore it as a strategy utilized by the potential audience to negotiate the paradox of women playing a hypermasculine sport. I will argue that this strategy works against the growth of professional women's hockey by both disavowing affect and ensuring women's hockey remains commercially non-viable. Lastly, I will argue in the next chapter that affective engagement is a key component when strategizing audience building. 


\section{Chapter 5 Discussions}

The previous chapter presented findings from the mixed method research on the women's hockey audience in Canada. This chapter will provide a discussion of these findings within the framework of the aims of the study, exploring implications and limitations, within the context of existing literature. The purpose of this study is to understand how audiences engage with women's hockey as a media text, how they accept, negotiate or reject messages of nationality and gender normativity, and to identify and understand the barriers inhibiting the growth of the professional women's hockey audience. The research questions recognize the power of sport media to perpetuate dominant social norms (Hutchins \& Rowe, 2012; Kennedy \& Hills, 2015; Raney \& Bryant, 2009) both in terms of representation (how certain bodies, situations and activities are presented) (Koivula, 1999; Kane, 1996; Messner, 1988; Wright \& Clarke, 1999) and visibility (Cooky et al., 2013; Cooky et al., 2015; Packer et al, 2014). My research recognizes hockey as a social discourse that actively works to construct a specifically gendered Canadian national identity (Allain, 2008; Gee, 2009; Gruneau \& Whitson, 1993; Watson, 2017). I seek to critically examine the implications of the exclusion of women players from the mainstream media discourse of hockey and consider reparations that may to lead to more equitable media coverage of the women's game and ultimately a stable audience and secure future.

\section{Women's Hockey Audience}

Who is the audience for women's hockey? The research question contains a number of complex components. Who is the audience that watches women's hockey now, but also, who is 
the audience that should or could watch women's hockey in some undetermined future? And what about the audience in absentia, who may eagerly watch women's hockey during the Olympics but can't be enticed to watch the professional women play? Is it even possible to conceptualize the women's hockey audience as a stable category in an increasingly fragmented media landscape (Livingstone \& Das, 2013)? Within the field of communication, the determination of what an audience is and what an audience does remains a source of debate (Das, 2019). Political economists (Smythe, 1977) theorize the audience as commodity, sought out, collected and gathered by broadcasters to be sold to the highest bidding advertiser. Social scientists (Hovland, 1951; Lasswell, 1927), concerned about harmful media effects approach the audience as passive dupes either lacking the skills necessary to critically analyze television texts or, perhaps, simply be unwilling to employ them. Critical cultural studies scholars theorize the audience as active, incorporating the engagement of television texts within their everyday lives for a wide variety of purposes and meanings (Ang, 1985, 1990; Hall, 1980; Morley \& Brunsdon, 1999). With datafication and mobile technologies, audiences are seen as agentic, mediatized consumers and producers of individualized digital texts "gaz[ing] at their computer or mobile phone screen, often alone, while multitasking social networking, music downloading, chatting, searching for information, or playing games and, simultaneously, discussing their experiences with others elsewhere" (Livingstone \& Das, 2013, p. 1), rather than collective entities with shared experiences actively engaging with mass media in the form of traditional, cable and satellite programming. Before examining the audiences for women's hockey, the first question must be: do audiences even still exist?

Livingstone \& Das (2013) argue for the continuing validity of the audience as both a practice, "audiencing" (Fiske, 1989), and an object of study. They approach the audience as a 
comparatively stable bridge between constantly changing and interacting texts and contexts, an activity made all the more visible given the multiple platforms available to viewers to express when, how and why they engage with media texts (Bird, 2012). Das (2019) argues for an increased interest in audience research as a way to analyze the datafication of everyday lives; utilizing audience research theory and methods can help the researcher learn if and how users may resist ubiquitous intrusions and constant demands for unpaid affective labour from powerful media corporations. Mediatization researchers do not always consider an active, critical and empowered user/audience (Couldry and Hepp 2018). If audience research offers value by providing a framework to examine user interaction with digital technologies and texts, are audience and users necessarily the same? Livingstone \& Das (2013) claim a deficit in the fluidity of this terminology, pointing out use reduces a text to an object that exists to have something done to it, rather than a site of interpretation and meaning making. Additionally, user represents a physical and temporal singularity with individual screens and media on demand and cannot encompass the collectivity captured by 'audience' (and by its contrasting alternatives - public, mass, crowd, market). Thus, the properties of collectives, and any (often rather unpredictable) power that thereby accrues, is missed by a reductively individual level of analysis. (Livingstone \& Das, 2013, p. 10)

The user/audience distinction is important to make in this study, especially when considering possible platforms for future women's hockey media distribution. Consider the 2018/19 season of the Canadian Women's Hockey League (CWHL). Of the 85 regular season games played, two were broadcast nationally on Sportsnet. Sportsnet also provided game coverage of the annual All Star Game and the Clarkson Cup Final. That is four out of 87 women's hockey games broadcast nationally on conventional television in Canada. This is the same coverage Sportsnet has 
provided since they first started broadcasting the CWHL in 2015 (Amanda Dwyer, personal correspondence, October 16, 2018). CWHL provided live-streaming of games on their website but this only covered 18 regular season games and three from the playoffs. Three days before the 2019 Clarkson Cup Final, the NHL Network announced they would broadcast the game to their American audiences; a gesture that would prove to be too late to save the CWHL (Rutherford, 2019). In the face of shrinking audiences, even for the broadcast juggernaut that is sport, is it even reasonable to think about professional women's hockey as a conventional television text?

Wall Street Journal writer David Pierce (2019) repositions the typical sport fan "plopping down on the couch — or, if you were lucky, in the stands — and watching a game" to an equally keen sports fan "checking reactions on Twitter, hunting for highlights on Instagram and sending OMG-that-shot faces on Snapchat" (1st. para). He explains that this is just the beginning of an eventual complete digitization of sport. In the near future, fans will be able to sit courtside, presumably somewhere near Drake, experiencing the sights and sounds, although not the smell, of sport through Virtual Reality (VR) broadcast. The economics here are clear: broadcasters and advertisers are slowly losing the battle to keep live sport feeds from non-paying streaming customers: "More than 460,000 people belong to a Reddit group devoted to streaming National Basketball Association games (without a license), and the BBC found that more than a third of Premier League soccer fans regularly watched 'unofficial' game streams" (Pierce, 2019, 3rd para). Given that VR headsets plug into conventional television systems rather than a live streams online, broadcasters hope to ensure that those who want the unique "game-changing" experience of sport on VR, must subscribe to an expensive cable package.

Survey respondents and focus group participants were asked to choose their preferred platform for watching women's hockey between digital streaming or conventional broadcast or a 
combination of the two. The majority $(85.9 \%)$ of survey respondents indicated their intention to watch the Canadian national women's team play Olympic Hockey on television, while 34.4\% said they would stream games online. In focus groups, online live-streaming services were frequently proposed as a solution to the poor mainstream media coverage given to professional women's hockey games. However, participants also felt that including women's hockey in traditional broadcast was necessary to "legitimize the sport as part of their regular programming" (C-3). Others argued that not all audiences are able to access online games: "it's not like, oh, there's all these people waiting to watch something. They can't access it. They're not waiting to watch it yet" (C-2). Participants articulated that a traditional broadcast assigns value to women's hockey, both in terms of marketing and publicizing the women's game and also legitimizing it to a mass audience as opposed to the individual user.

The purpose of this study is very specifically to study the audience for women's hockey as opposed to a user or spectator in order to explore the possibilities from the accrued power (predictable or not) that Livingston and Das (2013) argue comes from the audience as a collective property. The question in this study is not about whether audiences still exist, but is it possible to realize an audience as "public, mass, crowd, market" (Livingstone \& Das, 2013, p. 10) anywhere but necessarily within the traditional mass communication broadcast model? This is not to suggest that new media users lack connectivity, or agency or collective properties. Livingstone (2018) argues strenuously that while the practices of new media audiences and their interpretation of digital texts may have been sidelined in current mediatization research, scholars must remember to consider:

audiences' diverse interpretations of political media texts; their agency in acting on their interpretations as voters and citizens in a mediated world; the social and civic 
consequences of interactions among audience members; the difficulty of persuading audiences to think in particular ways; the aggregate effect of audiences' choice, search, selection, and commenting strategies; and the activities of audiences in creating and sharing mainstream, alternative, or resistant content. (p. 176)

Instead, the collective power of the mass media audience must be understood in both culturally and politically economic terms. The power of the audience as commodity for women's hockey must have enough of a use-value for producers to sell to advertisers to offset the always steep cost of sport production (Smythe, 1977). Producing sport is an analogue process requiring expensive stadiums, multiple cameras and crew, on-screen talent and infrastructure for a live audience. The financial investment is too great to take chances with the return. This is the classic chicken-and-egg argument that has limited media coverage of women's sports for decades: is it up to broadcasters to build an audience or do they simply respond to existing audience demand (Cooky et al., 2013; Cooky et al., 2015)? Broadcasters demands for a return on the investment required to produce sport becomes even less tenable in the relatively unstable market of online streaming. Unlike other digital content on YouTube for example, or Facebook, content that can be filmed in a living room with computer or cell phone cameras, the production costs for sport remain the same, regardless of platform. The investment reflects the content rather than the platform.

Academics and reporters have pondered the future of the conventional broadcast television economic model built on broadcasters gathering audiences through programming to sell to advertisers (Doyle, 2016; Ellis, 2000; Katz, 2009). Fragmented audiences, pirated programming and an abundance of alternative digital content, specifically social media and video games, has left conventional broadcasters facing revenue shortfalls. Still others remain 
optimistic. The Atlantic's Alexis Madrigal (2018) examines Nielsen ratings and concludes that “Americans still watch an absolutely astounding amount of traditional television” (3rd para), nearly 8 hours per day. Enli and Syvertsen (2016) observe "after a quarter of a century of "the end of television', it is interesting that there is still so much television left to debate" (p. 143).

With its reliance on a "live, unpredictable viewing experience" (Whannel, 2009, p. 205), broadcasters and scholars have argued that, with some adaptations, sport is uniquely able to withstand the transition from conventional broadcast to a digital streaming media environment (Billings \& Hardin, 2014; Zheng \& Mason, 2018). Taylor and Thomass (2017) credit the stability of the audience for the sustainability of televised sport:

Sport viewers are steadfast: they watch shows live, they rarely change channels during commercials, they gather in groups in front of the television, and they feel a strong emotional tie to the content on the screen. In an age of over-the-top (OTT) streaming sites like Netflix, and the threat of viewers cutting the cord to distribution services like cable and satellite, sports is seen as the content that will keep viewers tethered to traditional television services. (p. 112)

Whether the sport audience maintains its devotion to cable and satellite broadcast remains to be seen. Certainly they have shown themselves to be capable of a certain amount of fickleness. As discussed in the previous chapter, the Canadian men's national team is usually the Olympic ratings winner. At the 2018 Olympics, the overall audience numbers for men's hockey were much lower than previous years and the Canadian men's team bronze medal game ranked as the 7th most watched sporting event overall. Between the absence of professional NHL players and superstars and the team's failure to make the gold medal rounds, audiences for men's hockey proved to be more changeable than stable. The spring of 2019 is seeing a change in hockey 
audience behaviour. With no Canadian teams in the Stanley Cup playoffs and the Toronto Raptors basketball team winning the NBA Championship finals, Canadian audience numbers for men's hockey are have decreased: the NBA Eastern Conference finals garnered average minute ratings of 3.1 million while the Boston-Carolina Stanley Cup series was watched by an average of 1.3 million viewers. Compare this to the 2018 Stanley Cup round that featured two Canadian teams and were watched by an average of 2.5 and 2.7 million ((McGran, 2019). The question is: is the men's hockey audience, or even the sport audience in general, fickle enough to be enticed to become the audience for professional women's hockey? More pointedly, will the decreasing audiences for men's hockey lead broadcasters to lessen media coverage? Given the ideological rather than economic nature of ratings as Meehan (2002) argues, the answer is likely no.

Academics and reporters who write about the future of sport and audience consider whether those audiences can be maintained. Will they abandon their sport viewing activities? Will they stream games online (legal or illegally)? Will they pay large fees to satellite and cable companies so they can keep "a strong emotional tie to the content on the screen" (Taylor \& Thomass, 2017, p. 112)? Contemplating the future of an already existing entity is a far cry from thinking about building one from scratch. If professional women's hockey has struggled to build a conventional broadcast television audience, would switching to a digital platform make any difference?

I propose that the best way for women's hockey to build an audience is through conventional (satellite or cable) broadcast. As mentioned above, sport audiences can be fickle. Can existing audiences already consuming sport on satellite or cable, be drawn to watch women's hockey instead of, or in addition to, other broadcast sports? Certainly, the Canadian audience for women's hockey was trending upward from 40,000 in 2015 (Larkin, 2015) to 
175,000 in 2019 (The Canadian Women's Hockey League, 2019). Even though the CWHL has folded, there is value in speculating how the audience for women's hockey could grow. I argue that one of the findings of this study answers the chicken-and-egg dilemma between audience interest and broadcasters investment. Between the numbers of people who participated in this research study, the statements provided by respondents and participants, and the millions willing to watch Olympic women's hockey, clearly there is an interested audience for women's hockey, an audience-in-waiting, ready to be produced by a willing broadcaster.

Sport must be considered as a unique television text. Different from drama or comedy or most reality television, sport "audiencing" is a predominantly collective, preferably live, mediated experience. While the goal of this study is to examine audience engagement with hockey as a mediated text as opposed to spectating a live sports event, focus group participants frequently discussed watching women play hockey at an arena or community rink, both in terms of what they had or hadn't experienced and what they would or wouldn't attempt to do in future hockey watching. The distinction between a spectator at a live event, as opposed to a viewer of a mediated text, is important to make in the context of this research question that specifically queries the broadcast audience in terms of ratings rather than spectators at live games. I argue that, considering the development of the women's hockey audience, the experiences must be considered to be intricately connected and relational. As I have witnessed, and as was described by one focus group participant, producers of sparsely attended broadcast women's hockey games reposition the available audience to one side of the ice to display what appears to be a full arena for the cameras. In the documentary Play Fair (Gall, McIvor, \& Peeler, 2015), Olympian and former professional hockey player Hayley Wickenheiser comments that the growth of the women's hockey audience depends on "bums on seats" in arenas. In broadcast sport media, live 
spectators, individually and en masse, become valuable contributors to the text adding audio with crowd gasps and cheers from wins and losses and visual with audiences dancing, raising their arms in sequence to pass the wave and the ubiquitous kiss cam. Sport media doesn't simply reflect the live sporting event as much as it redefines it, in the case of women's hockey, repositioning subjects within the frame to support the narrative structure (Lever \& Wheeler, 1993). The strategy also circulates affect; the excitement of the live audience spreads to the television audience by example.

Diana-Luiza Dumitriu (2014) examines the structuring effects of the sport-media-nexus on the spectator experience to argue that mechanisms such as instant replay and micro close-ups have elevated mediated sport to become the preferred text for audiences. Viewers used to the focus and clarity that technological advancements have provided to game coverage may find the live arena experience less compelling and even less accurate than the mediated one. The dichotomy then is that the reliance on the live audience for women's hockey in order to make it a more valuable media text might be more difficult to build in the face of this theoretical rejection of the non-mediatized, or partially non- mediatized, live game.

Nevertheless, for advocates, the growth of the broadcast audience for women's hockey must begin with attending live games. Former CWHL Commissioner Brenda Andress founded the non-profit organization "She Is" (http://www.sheissport.com) with a goal to increasing viewership for women's sport that begins with increasing attendance: "the simple solution to support our female athletes is for you, the fan, to show up" (Brenda Andress, personal communication, June 10, 2019). However, as evidenced in this study, even though the majority of respondents and participants supported the idea of watching professional women play hockey, just under half had never watched a broadcast or streamed game, much less "shown up". 
Results of this research show the majority of participants who have watched women play hockey in the Olympics and/or professionally, self-identified as female with some connection to the game of hockey, as players themselves or through friends and family. Those who played women's hockey described their viewing activities in productive terms: they watch women play to get tips and to gain insight on certain game strategies and skills, appreciating or critiquing players' skating, passing and scoring with an insider expertise, distinguished from the less experienced, knowledgeable fan. For those focus group participants who played hockey and have seen live games, this conflation reached beyond cognitive practice. The feeling of playing, the sounds of the skates on ice, the smell of the arena is recognized in the media image, transported as it were through the television screen to the (experienced) viewers as though by "proprioception" (A-2). This will be explored further in the affect theory section below.

Association with hockey also included driving daughters and sons to and from practice, volunteering in leagues, and attending family members' games, arguably some of the domestic practices that Hardin and Whiteside (2011) identified as barriers to women watching women's sport. For some focus group participants, association provides access to and knowledge of women's hockey; for others, watching women's hockey becomes a gesture of support for their playing family member, a cognitive choice to legitimize the sport or a statement of advocacy. Unlike Hardin and Whiteside (2011), who found that access to sport viewing is often negotiated through male family members, participants in this study credited their daughters as the driving force to watch both women's hockey and men's hockey. This is an important consideration in audience building, especially given the growing number of girls signing up to play community or recreational hockey in Canada (Weir, Smith, Paterson, \& Horton, 2010; Wingrove, 2010) and in the United States (Murphy, 2018). These young female hockey players could be the realization 
of a promised, organically grown demographic who can demand that broadcasters and media platforms commit more, and more comprehensive, coverage. The CWHL recognized their future audience by providing free game tickets to members of community girls' hockey teams. The hope that hockey playing girls and women will save women's hockey must be tempered with an acknowledgment that the relationship is also symbiotic: participation grows in proportion to media coverage (CAAWS, 2016). While attractive to corporate funders, waiting for young female sports fans to mature into audiences may not be enough, especially given the lure of the ever-present, easily accessible and more highly prized men's hockey.

\section{Women and Hockey}

That women watch the game of hockey is not surprising despite Langley's (2003) argument that the National Hockey League (NHL) and Hockey Night In Canada (HNIC) negate and marginalize female viewing experiences and fail to account for any disparity in engagement that doesn't work to prop up, flesh out and homogenize expressions of nationalism and masculinity. While professional men's hockey broadcasts are characterized by a "pernicious sense of male entitlement: to space, to status, to national belonging" (Adams, 2006, p. 71), there have been some efforts to diversify at least the racial monoculture of HNIC, specifically with the long-running successful spin off Hockey Night in Canada Punjabi (Szto \& Gruneau, 2018). However, NHL/HNIC has not seen a gendered equivalent with the exception of the occasional inclusion of female commentators, such as, Cassie Campbell, Leah Hextall, Tessa Bonhomme and Andi Petrillo. Phillips (2018) recounts that the identification of women as a valuable hockey audience demographic coincided with the first woman sports announcer to appear on HNIC, 
Helen Hutchison in 1973, but argues the connection between the two is unconvincing. Hutchison's term lasted just one year and it would be another twenty before the HNIC brought in another female sportscaster, yet female hockey audiences continued to grow.

Phillips (2018) proposes that female hockey viewers in the 1980s were drawn in part by the unlikeliest of lures: Don Cherry and his inter-period commentary on Coaches Corner, known as much for his controversial pronouncements on society, gender and politics as hockey. Cherry has been associated, and associates himself, with women's hockey and its legitimacy, known by some to be a "strong supporter of the female game since the early 1980 s and ... to speak out in favour of women's hockey" (Canada's Women's Olympic Team, 1997), despite being called out for advocating a ban on female sportscasters in the hockey locker room (Power, 2013), and derided for remarks such as calling "the American players 'slobs'... noting that the Canadians looked like fashion models" (McKeon, 2018). His appearance during the 2018 Olympic women's hockey gold medal game received little scrutiny in the press but was a controversial topic of conversation in this study's focus groups. Some participants felt Cherry's appearance had a positive impact "because if Don Cherry is there that might draw a new audience or a bigger audience in, even though we may not love him, people do associate him with hockey" (B-2). Others were affronted by his appearance claiming "he kept talking about the men's game, didn't really know who the players were in the women's game. It was an embarrassment to see him speaking about women hockey. Something it seems that he knows nothing about” (B-4). Participants felt that Cherry's avuncular appearance was devised to lend credibility to the women's game, a blessing of sorts, a statement of legitimacy, authenticity and recognition even as they expressed their own rejection of the man and his association with the women's game as inappropriate. 
This is supported by researchers who argue that commentators wield great influence over how audiences interpret sporting events and athletes (Bryant, Comisky, \& Zillman, 1977; Tudor, 1992), constructing discursive frameworks that often serve to reinforce ideological norms, particularly in regards to race and gender (Billings \& Eastman, 2002; Duncan, Messner, Willms, Wilson, \& Amateur Athletic Foundation of Los Angeles, 2005). Kelly Poniatowski and Marie Hardin (2012) argue that the NBC commentators for 2010 Olympic women's hockey, while professing support for the game and its players, habitually "position female players as substandard (with the standard as masculine) in several key ways. Thus, it ultimately reflects a strategy of ambivalence that undermines the credibility of the athlete and women's participation in the sport" (author's italics, p. 624). Poniatowski and Hardin (2012) do not make an explicit connection between this "ambivalent" commentary and the gender of the media personality giving it, other than to note that of the five commentators analyzed, two were women.

Whether Cherry's plain-talking everyman approach to hockey, his flamboyant costumes, outrageous statements and his "cult of personality" led to an increase in female viewership is unknown. Phillips (2018) suggests that Cherry was perhaps "a happy accident" in CBC plans "to attract more prime time female viewers via more widely accessible content, while not alienating its already established male prime-time audience with more coverage of women's hockey, or else with non-hockey content” (p. 213). Certainly, this is supported by Meehan's (2002) argument that the primacy of the male audience demographic is always secured, even at the risk of lost revenues from an untapped female market. It is equally conjectural that the male hockey fan might boycott their Saturday night ritual (Langley, 2003) in the face of a gendered infiltration. More specifically in need of interrogation is the idea that female hockey fans might only be drawn in by "female-friendly hockey sub-content" (Phillips, 2018, p. 212). This implies that 
female audiences are unable to make a connection with the technical and strategic components of the game, that the language of hockey is necessarily foreign to the female viewer. However, Crawford and Gosling (2004) found female fans of ice hockey in the U.K. to be both knowledgeable and engaged with the details of the games and players, despite the concerted efforts of male fans to marginalize and dismiss them as "Puck Bunnies". The presumed homogeneity of the sport audience has been challenged by scholars who identify a wide array of racial, gender and class identities beyond white males, as well as a diversity of reasons for engagement from strident fan to casual viewer (Caudwell, 2007; Cohan, 2019; Schirato, 2013; Szto, 2016; Theodoropoulou, 2018; Toffoletti, 2017; Toffoletti \& Mewett, 2012).

Many of the female focus group participants in this study spoke of their interest in, knowledge of and passion for the game of hockey, often regardless of player gender: "you know, my dad and I will put on whatever game is on that night and watch it and enjoy it because we love the sport" (C-5); "for me, hockey is hockey and I enjoy watching it ... men's versus women's, well it's all just a good game and I love watching it" (B-3). The female sport viewing experience may be multi-layered and nuanced, as I'll discuss below, but it is important to state that the most basic component of knowing and enjoying game play is included. Insistence that women's sport spectatorship requires some level of interpretation or filter outside of, or in addition to, watching gameplay works to construct an identity devoid of expertise and authority and further marginalizes their experiences (Crolley \& Long, 2001; Farrell et al., 2011; Kissane \& Winslow, 2016; Markovits \& Albertson, 2012). Even an initiative such as Toronto's The GIST (http://www.thegistnews.ca), a female-run website providing sport news specifically for women, includes a separate page detailing the basic rules of major sports. The assumption is clear: women as aliens in a foreign landscape require a lesson in sport as a second language. 


\section{The Female Sport Audience}

Scholars are showing increased interest in the female sport audience in general with particular attention paid to the female sport fan (Farrell et al., 2011; Gosling, 2007; Hoeber \& Kerwin, 2013; Osborne \& Coombs, 2013; Sveinson \& Hoeber, 2015). The focus for many researchers considers how female sports fans are: persistently marginalized in the hypermasculinity of most sporting cultures (Jones, 2008; Pope, 2011; Sherlock \& Elsden, 2000); their heterosexual interests in male athletes questioned and rejected (Esmonde, Cooky, \& Andrews, 2018); and their interest in sport reduced to satisfying the desires of their male partners, fathers and siblings (Farrell, 2006; Whiteside \& Hardin, 2011). Hynes and Cook (2013) explore how female fans go online to express their sporting interests, in blogs and on social media groups, often hiding their gender to sidestep the barriers they face attending live games, including access to arenas and performances of masculine violence in the stands. Female sport fans are expected to be knowledgeable than their male counterparts or face accusations of inauthenticity, only then to contend with the fine line between knowledgeable and unfeminine 'know-it-all'. Sveinson \& Hoeber, 2016 argue that viewing sport can be an empowering experience for female fans, especially when they demonstrate knowledge and competency that defies dominant perceptions that their fan experience is inauthentic. Empowerment from watching women's hockey was discussed by focus group participants, often articulated as the expected outcome for young girls learning from women hockey players as 'role models'. For participants, watching women's hockey becomes an empowering political act of solidarity and defiance against gender equity, both on the ice and off. 
Much of the existing research focuses on non-spectatorship presents data showing the small female fan base for women's sport (Farrell et al., 2011) and examines how women don't watch other women play sport, particularly team sports that involve physical and aggressive play that contravene gender-role expectations and are deemed inappropriate for women (Costa \& Guthrie, 1994; Lee, Zillmann, \& Waver, 1998). Antunovic and Hardin (2012) focus on femaleled blogs about women's sport and find sentiments that support the dominant gender ideology of sport that values male athletes over female, demonstrating that female bloggers desired Women's National Basketball Association (WNBA) players to aspire to the excellence of the men in the National Basketball Association (NBA). In this study, focus group participants who identified themselves as non-spectators offered an alternative explanation, most frequently pointing to a lack of information about the days, times and platforms of broadcast games. Mainstream sport media company infrastructure and daily sport news segments as well as insufficient league publicity strategies were critiqued as barriers that excluded participants who would otherwise be willing to tune in or attend games.

Female fans and spectators of women's sport have received little academic attention. Toffoletti is one of the leading scholars on women sports fans with publications including Sport and its Female Fans (2012), a book she co-edited with Peter Mewett, and her most recent book, Women Sport Fans: Identification, Participation, Representation (2017). Toffoletti uses a number of theoretical lenses including post-feminism, neoliberalism, consumer citizenship and transnational feminism to position her research, and honour the place that sport holds, in the everyday lives of women around the world. Despite acknowledging the dearth of academic research focusing on women fans in general and particularly women fans of women's sport, only one chapter in Sport and its Female Fans offers analysis of female fans for women's sport. 
Lindsay Meân (2012) conducts a comparative analysis of the WNBA website with the (men's) NBA website to show how digital sport design and content perpetuates gender inequity. Toffoletti limits her own engagement with female fans of women's sport to the last chapter and a half of her 2017 book. In one chapter, she interrogates how female fans use social/mobile media platform to promote women's sport. She focuses on a women's soccer tournament in Australia, the Asian Cup, to consider how female Australian-Iranian fans used Twitter to distribute images of themselves at matches, in full game regalia, to women in Iran who are not allowed to attend sporting events. Toffoletti argues the circulation of these affectively empowering images serves to create collective and community identities for Iranian women soccer fans and solidarity with women sport fans more generally around the world. Toffoletti focuses on the media coverage these tweets received arguing that reporters were less interested in touting social media's potential for female fan collectives than they were in trumpeting the freedom westernized Australian-Iranian soccer fans enjoy in comparison to their oppressed sisters in Iran. Tinged as they are with a nationalistic superiority, Toffoletti (2017) warns these reports "threaten to overshadow the potential of social media for advancing transnational feminist alliances and advocacy around gender justice in sport by evoking modernist myths of western progress (sporting, technological) as a facilitator to gender equality" (p. 113).

Toffoletti goes on to propose a double-sided typology to characterize the media representation of female fans: the post-feminist female fan. In this binary, on the one hand, female sports fans are celebrated for their physical attributes and their competency; performing personal and sexualized empowerment as a neoliberal choice, even as it functions to strip their authority and authenticity and re-inscribe sport sexism. Toffoletti focuses on the 2015 FIFA Women's World Cup to identify an alternative image of empowerment for the female sport fan, 
"that of the 'future girl' (Harris, 2004) or 'top girl' (McRobbie, 2007) who is capable, competent and embodies the potential and capacity of young women everywhere" (Toffoletti, 2017, p. 124). While not discounting the possibility that media representation of the female fan for women's sport may also include sexualized imagery, the latter definition holds the most potential to inform this research study by providing insight into the constitutive power of media representation and participants' conflation of "audiencing" with political/social activism discussed in the previous chapter. I'll begin as Toffoletti does with an exploration of postfeminism.

A multiplicity of definitions make postfeminist concepts particularly challenging to interrogate. McRobbie $(2007,2009)$ critically identifies postfeminism as a term of redundancy that presumes the goals and ambitions of liberal feminism to be achieved so as to no longer be of use, patriarchal institutions presumably made inert in the face of autonomous, empowered, individual women (also Gill, 2007), now free to define their femininity through freedom of choice in the neoliberal marketplace (Gill, 2008; McRobbie, 2009; Negra, 2009). In this way, gender discrimination becomes a matter of individual responsibility rather than systemic inequity. Postfeminism can also indicate a shared co-existence, or even addendum to, liberal feminism; one that promises an intersectional corrective to the traditional white, middle-class, heteronormative stronghold on the definition (Vered \& Humphreys, 2014).

Sport scholars (Cole \& Hribar, 1995; Francombe, 2010; Heinecken, 2016; Lafrance, 1998; McDonald, 2000, 2012) identify a particular enactment of postfeminism in sport that elevates (literally on a podium) narratives of individual triumph/empowerment and defines emancipation through participation in the sport market as spectators. Toffoletti (2017) suggests that when the spectator watches women's sport, the specific representation of the ideal fan 
constructs an identity that disavows a stereotypical sexualized femininity in favour of an active, educated, egalitarian femininity:

Decked out in team colours, this female fan embodied a related, yet sufficiently distinct, form of contemporary femininity to her 'sexy sister' in both age and looks. Commonly imagined as a girl, tween or teenager, she frequently appears amidst a group of female friends - a celebration of sporty sisterhood, if you like - clear eyed and fresh faced, long hair pulled back in a ponytail or contained by a colourful headband. (p. 141)

Toffoletti refers to Harris' (2004) "can-do" girl as the foundation for this idealized female sports fan as empowered, confident and enthusiastic about her place in the world and her unlimited future as capitalist consumer. Often framed against the "at-risk" girl, the "can-do" girl participates as player or fan in any sport as though by choice, sidestepping any institutional, economic and social barriers that may have limited her foremothers. I argue that sport becomes not just an expression of "can-do" girl empowerment, but instead becomes the reason why she is empowered. Sport is framed as protection, ensuring the "can-do" girl does not become "at-risk". In contrast to the sexy female fan of men's sport who is afforded empowerment through representation of physical attractiveness, Toffoletti argues that the "can-do" fan's prominent girlhood defines her future role as market consumer and audience commodity to "sustain the market privilege and broader social power and influence wielded by dominant institutions and organizations, such as FIFA" (Toffoletti, 2017, p. 142). The postfeminist poster child of selfdetermination and sufficiency also suggests unhindered intersectionality with images of diverse "can-do" girls, sport's multiculturalism touted as progressive even while perpetuating narratives of colonialist marginalization that sees girls of colour needing sport to rescue them from precarious situations (Hayhurst, 2014; McDonald, 2015). 
Surprisingly, given the space dedicated to the sexualized postfeminist sport fan here and in earlier her work on the female fan's objectification of the male athlete, (Toffoletti \& Mewett, 2012), Toffoletti fails to consider how youthful representation of the "can-do" girl also works to actively de-sexualize female fans of women's sport as a function of systemic homophobia and gender normativity. When it comes to expressing sexual attraction between fan and player, the "can-do" girl doesn't and this, in essence, neutralizes the homophobic threat of lesbianism that is constructed to be a barrier to media coverage and audience growth. I indicate this as a perceived barrier constructed by broadcasters that they require to be resolved; the empirical data from this audience research study does not support this as a specific articulation. However, I note a number of scholars examining issues of homophobia in sport such as Davison (2009), Stoelting (2011) and Waldron (2016) and acknowledge persistent anti-gay discrimination and heterosexism in men's (Parviainen, 2018) and women's sport (Blinde \& Taub, 1992; Cahn, 1994; Griffin, 1998) and will argue below how concerns around sexual orientation become articulated as expressions of femininity.

\section{Hockey and Gender}

While some focus group participants spoke of how playing hockey led to homophobic slurs, "if you were a good player, you'd have lesbian or gay stamped on your forehead" (A-3), women's hockey and its audience were more frequently and positively positioned as being inclusive in terms of gender identity and sexual orientation: "I'm okay with women's hockey and just knowing that it's inclusive, but there is an argument to be made maybe going forward to relabel that 'women and trans hockey', I think it's just non-CIS male hockey" (A-1). Some 
participants made a connection between the historical perception of sport as "unfeminine" (F-2) and the lack of audience engagement and institutional financial recognition but this was not specifically defined in terms of player or fan sexual orientation. For these participants, hockey was the key to broadening and re-defining those traditional, patriarchal notions of femininity. Re-defined perhaps to incorporate strength, aggression and physicality, femininity as embodied by the female hockey player — " "a young woman in her hockey gear, taking her helmet off ... the flushed cheeks ... beautiful" (F-2) — imbues her with an unquestionable authority to play role model to young girls and women. Further, it is through the establishment of players' heteronormative attractiveness, despite their strong bodies engaged in masculine-coded play, that participants find encouragement for the eventual legitimacy of professional women's hockey. In this way, definitions of idealized femininity may be expanded to include fitnessdriven activities yet "idealized masculinity" (Antunovic \& Hardin, 2015) as gender norm is maintained.

The association between women's hockey and social value was articulated by focus group participants as a source of possible social and political benefits associated with liberal feminist goals, specifically around issues of equal pay and representation. In their analysis of women-generated online sport blogs, Antunovic and Hardin (2015) identify a "feminist approach to both sport participation and fandom" (p. 673) drawn from a negotiation between Coakley's (2004) two theoretical frameworks that conceptualize sport as either a hypermasculine competitive environment where winning is prized above all else; or, an active participation structured around and valued for forming connections that "encourage inclusion and cooperation rather than exclusion and hierarchical arrangement of dominance" (Antunovic \& Hardin, 2015, p. 665). While radical feminists (Miner \& Longino, 1987: 256) reject competition as an 
expression of masculine dominance, Antunovic and Hardin (2015) propose Lenskyj and Sport Canada's (1994) framework as a complement to the either/or dichotomy of competition and connection. Their hybrid feminist framework considers how women's participation, as players and fans, may challenge "the dominant value system [that] promotes dominance over others, self-sacrifice and hostility toward the fellow competitor" not by negating concepts of competition but reframing the desire to win as an expression of "empowerment, skill enhancement, and connections" (Antunovic \& Hardin, 2015, p. 673).

This framework becomes valuable when examining the proposition that engaging with women's hockey as sport and as media text is a fundamentally feminist act. As discussed in the findings chapter, focus group participants described their reasons for watching women's hockey as a way to express their support for gender equality, to feel a sense of empowerment, to provide strong role models for girls and to recognize a shift in society:

I think there's some real fertile ground right now, like with all of the advances you know, with all of the movements that are happening right now, like surrounding feminist and young women like declaring themselves as feminists and women CEOs and women politicians with all of that. I think there's a real fertile ground for the CWHL to just explode and also the skill in women's hockey, women's sports in general. (A-1) They seamlessly negotiate with the competitive elements of hockey, cheering their favourite team or player on to win, yet in a way that is subservient to the greater good of participating in women's quest for gender equity. They decry moments when female athletes are celebrated for their looks instead of their athleticism; they critique inequitable access to rinks and ice time; they resent limited coverage on daily sport media. Even the physicality of the game, the bodychecking and scuffles associated with the violence of the men's game, is re-framed as evidence of 
legitimacy: "anyone who watches it knows the physical contact, the strengths and power of these athletes is incredible, nobody's going to say that those girls aren't throwing body checks and are getting penalized for it and throwing punches" (E-3). For A-2, the physicality of the game is seen to provide opportunities to build close connections:

I never would have had the confidence to relate to another woman like that. You're bumping up against your girlfriends; there's a physicality that I, even in my theatre days, rolling around and dancing, we never had that same kind of physical, that sort of jocular, teasing in the locker room.

Even something as drenched in patriarchal villainy as locker room talk (Kane \& Disch, 1993), can be re-framed as a feminist consciousness-raising session. Consistent with Kniffin and Palacio's (2018) findings about trash talk and gendered athletes, female participants' descriptions of on-ice trash talk went no further than curses over a lost goal; rather, appreciation for an opponent's well-timed slap shot was more commonly expressed.

\section{Women's "Pure" Hockey}

Conversely, focus group participants frequently touted the women's game for its superiority to men's hockey for being less violent and more skill-based: "not to say that the women's game isn't physical, it certainly is, but it becomes more of a positional game. It's about passing lanes, it's about systems and it's the speed of the puck. It's what hockey should be" (B1). Appreciation for game play is interpreted as evidence of players' skills, connections and teamwork, particularly in contrast to men's hockey with short shifts for players racing to get in front of the net, "the rock star coming down the ice and getting all the glory" (A-2). Men's 
hockey then is actually antithetical to teamwork; players must compete against teammates as well the opposing team in a quest for game points, salary and media exposure. Teamwork in women's hockey is articulated as evidence of a morally superior commitment to community responsibilities as opposed to selfish individualism and feeds into traditionally feminine representations of women as caring, self-sacrificing and maternal. Participants view men's hockey as rough, violent play where fights are prized as much as goals; in contrast, they describe the women's game with a sepia-tinged temporality, a "pure" version of the game as it used to be played before its current iteration as economic juggernaut of the sport/media complex. The female athletes then become the enactment of the hockey myth, keepers of the faith, players in it for the love of the game rather than the size of their paycheque. This perception bears some scrutiny. As Phillips (2018) argues that men's professional hockey from inception, has always been a commercial venture. As early as the 1920s, NHL games were regularly covered by radio broadcasts; by 1929 McLaren Advertising was selling sponsorship dollars and two years later, the games — including its advertisements — reached over 1 million Canadian listeners. When the NHL struck a deal with the newly formed Canadian Broadcasting System in 1936/37, Imperial Oil was the program sponsor for radio broadcasts reaching an audience of 2 million Canadians every weekend (Scherer \& Whitson, 2009). By the early 1960s, televised HNIC games sold advertisements to an audience of 5.5 million (Scherer \& Whitson, 2009). Far from a pure love of the sport, professional men's hockey has always been monetized and structured as capitalist spectacle from its very beginnings.

Yet, the mythology persists, enacted and re-enacted in discourses around women's hockey. Much as Jameson (1991) described nostalgia films less interested in representing a historical period in its "lived totality" than "reinventing the feel and shape of characteristic art 
objects of an older period, [that] seek to reawaken a sense of the past associated with those objects" (p. 8), women's hockey works as a characteristic art object, or placeholder for a history of hockey that never was. While Adams (2006) identifies hockey nostalgia as an instrument of exclusion, a way to ensure its male whiteness remains intact, the ideological work with women's hockey is different. First, the discursive construction of women's hockey as "pure" protects the integrity of hegemonic femininity, most at risk in this hypermasculine sport. Women's hockey is effectively feminized, saturated with hegemonically feminine values of virtue, purity and morality. Secondly, the representation of non-commercial hockey, be it amateur or noncommercially viable hockey, in such temporally fluid terms (e.g. what men's hockey used to be) works to reinforce the mythological foundations of men's hockey in Canada and even faintly promise its return. If women's hockey is what men's hockey used to be, maybe men's hockey will be that way again too:

I think the women's game is becoming more like the men's game, but more interestingly the men's game is becoming more like the women's game. I don't think they'll ever converge, but we're at a period of time now where the two games are becoming more and more alike. And I think that the reason for that, especially on the men's side, is that the women's game is the pure form of hockey. It's really what hockey is as, as a sport. (B-1) Lastly, nostalgia ensures women's hockey and its players remain locked in exhausted combat with an un-winnable myth rather than working to defining their own iteration of the game. Bélanger and Valois-Nadeau (2015) turn to Barthes Mythologies (1972) to interrogate how the sentiment of a "pure" hockey informs the images and discourses that surround the Montreal Canadiens and their Quebecois fans constructs hockey as a naturalized expression of Canadian (Quebecois) identity. Barthes explores how myths work to naturalize perceptions by 
presenting values or ideology as if they were natural conditions of the world rather than social constructions to support power structures, benefiting some while excluding others. Barthes refers to Saussure's (2006) foundational work in semiology that approaches language not as a fixed object but as a social process that carries multiple and changeable meanings, with no intrinsic link between signifier and signified. He argues that myths construct meaning at a secondary level through the re-signification of an already constituted sign by way of distortion, to be perceived rather than interpreted and accepted as true.

Bélanger and Valois-Nadeau (2015) identify the paradox between the image of the shinny-playing child on a Canadian frozen lake celebrating the country's sturdy citizens "untainted by aspirations of salary and upward mobility, the child plays just for the fun of it, with his whole heart, and to defend the honour and pride of his team and village" (p. 71-72) and the game infected with commercialization of the sport and its "insidious effects" (p. 71). The mythology of hockey that solves this paradox is created through the calculated, coordinated and collective efforts of three entities: the market, the media and the fans who create and re-create images and narratives to obfuscate the origins and continuation of hockey as a commercial, profit-driven venture. The myth guides its self-sustaining circularity:

Had there been no radio play-by-play, had there been no television coverage, hockey could never have infiltrated the daily lives of millions of Quebecois. And without these entertainment platforms there to fuel the pride of an entire people and galvanize it in support of the Montreal Canadiens, the team could never have grown into the national emblem it is today" (Bélanger \& Valois-Nadeau, 2015, p. 84-85).

As the men's professional hockey league develops and moves further and further away from its mythical origins, fans double down on their efforts to restore their familiar and personal 
associations to the game by "rehashing the so-called 'original' values of yesterday's good old boys" (Bélanger \& Valois-Nadeau, 2015, p. 90).

For focus group participants, positioning the women's game as representing those "good old boy" values suggests an attempt to convey a legitimacy that goes beyond the morally superior game play from less violence and more teamwork. There is a specific historicity at play here. When A-2 identifies the romance of the original six hockey teams of the NHL as "this idea that authentic hockey is the original six and we play like they do", even while sidestepping the scars, stitches and missing teeth of those un-helmeted players, the intent is to right the historical wrong of women's exclusion from the game, to correct Gruneau and Whitson's (1993) “cultural amnesia about the social struggles and vested interests—-between men and women, social classes, regions, races, and ethnic groups - that have always been part of hockey's history" (p. 132). The belief here is not only is women's hockey is as good as men's, it is superior for having resisted the crass commercialization suffered by the NHL and the accompanying degradation of the game.

The paradox is the elevation of women's hockey for its adherence to "pure" (i.e. noncommercial) game play becomes the argument for why it should be considered as a more commercially viable product. Focus group participants routinely positioned the women's game as different and superior to the men's as a way of advocating increased audience size and financial compensation for the players as evidence of social legitimacy. This is done by imbuing women's hockey with a sepia-toned romanticism of the original six that both supports the myth of hockey as a "pure national symbol", although here long skirts and ice skates take the place of the boy on the frozen lake, but also simultaneously eradicates women's own long history, not just in the sport but as paid players in a professional league. Denied commercial viability, 
professional women's hockey remains amateur, as though for its own good.

\section{The Professional Amateur}

In his 1978 essay, Sport and Social Class, Bourdieu exposes the foundations of the amateur ideal of sport as an expression of elite class distinction. What were once games for the unwashed masses, sport in the leisurely hands of the aristocrat becomes:

disinterested practice, a finality without an end, analogous to artistic practice, but even more suitable than art ... for affirming the manly virtues of future leaders: sport is conceived as a training in courage and manliness, 'forming the character' and inculcating the 'will to win' which is the mark of the true leader, but a will to win within the rules. This is 'fair play', conceived as an aristocratic disposition utterly opposed to the plebeian pursuit of victory at all costs. (p. 824-825)

The tension Bourdieu notes is in the hierarchical distinction between sporting activities, some coded as acceptably refined (often with accompanying clubhouses) compared to the vulgarity of other practices more suitable for the lower rungs of the social hierarchy. He also notes a difference in the intention of sport practice from filling aristocratic "spare time" compared to working class aspiration via the court, the field or ice. While the elite amateur competes for personal best or possibly reaffirmation of social status, working class athletes contend with different stakes: "The working-class cult of sportsmen of working-class origin is doubtless explained in part by the fact that these 'success stories' symbolize the only recognized route to wealth and fame" (Bourdieu, 1978, p. 832-833). Positioned in contrast to the entitled amateur and his abundance of spare time, the working class athlete instead must be compensated for 
sporting time that would otherwise be spent earning on the factory floor. The professionalism of sport, meaning the working class athlete is paid, requires its commercialization. Kidd (2013) makes the distinction clear when critiquing the commercialization of Canadian hockey:

Commercial hockey in Canada is called 'professional' hockey, but strictly speaking the two terms are not synonymous. In 'professional' hockey, the players are remunerated for their hockey efforts and thus can be distinguished from 'amateur' players who are not so remunerated. The term 'commercial' refers to a different aspect of the game altogether, the ownership and control of the game. In 'commercial' hockey, team owners enjoy complete property rights in the players and the right of admission to actual contests, and they operate the hockey enterprise to make a private profit. It has been the commercialization of the game, not its professionalization, that has harmed Canadian hockey. (p. 353)

While Kidd argues that professional, non-commercial hockey players may be remunerated by the gate, this hasn't been the case for women's hockey that, as noted earlier, has struggled as much with "bum-in-seats" as with broadcast audience.

Travers (2008) critiques the economic structural disparity of sport as a particularly pointed expression of gender injustice. The cultural effects of a two-sex based system that normalizes masculine superiority and relegates other genders to either the margins (women) or obscurity (transgender and non-binary athletes) plays out in a self-sustaining feedback loop in which the cultural celebration of the male athletes leads to economic awards which leads to further cultural celebration and so on. The affirmation then is not only in a gendered hierarchy but in a naturalization of the binary itself. Travers considers strategies proposed to correct the gender injustice of sport, many associated with specific iterations of feminism. These include a 
radically feminist "top down" replacement of sport's gendered hierarchy with "cooperative and non-hierarchical celebrations of physicality and play based on feminist principles of cooperation and inclusion" (p. 86); a third wave feminist approach that also recognizes and celebrates individual athletes who trouble gender normativity, change coming as it were, from the bottom up; a post-feminist vision that removes gender distinctions in favour of sport as meritocracy; and the liberal feminist/queer intention of equity that "eliminates male-only sporting while maintaining sporting spaces for girls and women” (p. 86). Travers favours recommendations that radicalize a liberal feminist quest for equity with a recognition that participatory parity must be intersectional and demands the rejection of the biologically essentialist hierarchy implicit in the gender binary.

I observe that the recommendation Travers (2008) identifies as a third wave feminist focus on athletes who disrupt discourses of gender normativity enacts Itani's (2015) caution that intersection be not a "dominant-centric additive of the marginalized, but a destabilization of the centre - a deconstruction of women, men, sport, nationalism and colonialism" (p. 264-265). Itani and other scholars (Cronin \& Armour, 2015; Clark, Ferkins, Smythe, \& Jogulu, 2018) argue that the experiences of athletes must be made central, their voices heard and projected through scholarly work. Focusing on the experiences of athletes who agitate against gender normativity also satisfies Markula's (2003) interpretation of Foucault's technology of the self. Markula argues for the transformation of sport from within that depends particularly on athlete's critical self-awareness to resist and trouble the power relations of sport. In this context, I argue that the 200 professional women hockey players who have unionized and planned a boycott of this upcoming season (Knight, 2019) represents such a critical self-awareness. Whether the outcome leads to a transformation of sport power relations (possibly one gender-inclusive league, player 
run and economically sustainable) or a reaffirmation of structural gendered hierarchy (professional women's hockey as a subsidiary of the NHL) remains to be seen.

\section{Inclusive Women's Hockey}

As mentioned in the previous chapter, the results of this study indicate a demographic homogeneity in the sample. This is evidenced particularly in the gender of respondents with the majority defining themselves as female/CIS-gendered female. The other notable and troubling result is the lack of racial or ethnic diversity in the respondents with $90.0 \%$ identifying as white. While this may very well be a failing in recruitment, I argue this indicates a broader issue of lack of diversity in sports in general (Andrews, 1996; Farred, 2018; Grainger, Newman \& Andrews, 2006), and hockey in particular (Abdel-Shehid, 2005; Cairnie, 2019; Krebs, 2012; Poniatowski \& Whiteside, 2012) that either exclude non-white bodies or frame non-white athletes in ways that reinforce racial stereotypes and discriminatory practices. Pitter (2006) recounts a long history of black and aboriginal hockey players in Canada, although ignored and made invisible in popular hockey mythology, and the racism players of colour continue to experience on the ice and in the dressing room. He cautions that: "ignoring the accomplishments of non-whites in hockey, as well as the obstacles they have had to struggle against, has served to distort our collective consciousness" (Pitter, 2006, p. 135). Adams (2006) argues that Canadian men's hockey, in its lack of racial diversity and its conflation with nationalism, serves to construct and

"keep[s] whiteness central to dominant notions of Canadian identities" (p. 76). The centralization of hockey as a national discourse ensures that those who the discourse privileges, "native-born, 
white men" (Adams, 2006, p. 71) remain central. Other lives and experiences are kept off the ice and in the margins.

There has been little academic research in women's hockey and racial diversity (Danford \& Donnelly, 2018). Media reports have highlighted the lack of representation of non-white players on the professional circuit (Ayala, 2017). Angelica Rodriguez (2017), a writer with the feminist sport publication The Victory Press, conducted a roundtable discussion with women of colour associated with women's hockey to talk about experiences of racial discrimination and bias, including denied opportunities to play on national teams and coded language to ensure marginalization: “often, 'attitude' and 'chemistry' are cited as reasons for P.O.C. not to be allowed a chance to play in national events, which is code for race and perception of race" (Rodriguez, 2017, 9th para.). Even as the NHL touts the economic importance of multiculturalism and diversity (Frey \& Davis, 2018), and the website The Color [sic] of Hockey (http://www.colorofhockey.com) celebrates growing diversity in community leagues, the touted inclusiveness of elite women's hockey (Murphy, 2017) appears to be incomplete.

With the exception of the pre-contact shinny played by aboriginal women (Hall, 2002 and see Hall, 2013, 2016), the history of women in hockey in Canada has been a story of able-bodied white women, initially working class (Hall, 2016, p. 22), but more recently middle and upper class, with costs of gear, league fees and ice time being prohibitive for many (Kirby, Letts \& Steckley, 2013; Mirtle, 2008). Hall (2016) recounts the difficulty finding and accessing historical documents relating to the history of aboriginal women in this sport and argues that simply plugging them into the existing historical narrative neglects the autonomy of their specific experiences. Others (Vertinsky, 1994; Ellison, 2002) critique the lack of historical research focused on Canadian women athletes of colour beyond an acknowledgement that few women of 
colour were allowed to participate in sport until the last half of the twentieth century, and even then, they faced discrimination and social and economic barriers to sport (Burnett, 2001). Stories of women like Angela James are beginning to be told. Angela is one of the first two women to ever be inducted into the Hockey Hall of Fame in Toronto, the first openly gay player and the second black athlete ever to be inducted. In February 2019, Sportsnet featured her story in a short video and article in celebration of Black History month (Sadler, 2019). In 2018, Brigitte Lacquette received extensive media coverage as the first aboriginal woman to play on the Canadian Olympic women's team (Arthur, 2018; Bellegarde, 2018; Pimental, 2018). While Canada has a national women's sledge hockey team that competes internationally, women's sledge hockey has not been recognized by the Paralympics and is therefore not funded by Hockey Canada (Syal, 2018). Future academic research in women's hockey must consider intersectionality in telling the history and present-day conditions of players of all abilities, ages, genders, classes and races.

The lack of diversity in this study and in women's hockey contradicts the statements of many of focus group participants that characterize women's hockey as a fundamentally inclusive sport, often presented as a distinguishing feature compared to the men's game and contributed to the perception of women's hockey as “pure” and morally superior. This was specifically articulated in discussions about the inclusion of transgender players in the Canadian Women's Hockey League (CWHL) and the National Women's Hockey League (NWHL). Jessica Platt joined the CWHL team, the Toronto Furies in 2016; two years later, in January 2018, she informed her teammates she was a transgender woman and came out to the public on Instagram (Brady, 2018a; Bennett, 2018). She is the first and only 'out' transgender woman on the CWHL and has publicly acknowledged the league for providing private and public support to her 
throughout (Brady; 2018; Bennett, 2018). For some focus group participants, this acceptance led them to conclude that women's hockey is open and non-judgemental in a way that is unique in sport generally and hockey in particular. A-1 even suggested a category change to reflect the sport's inherent inclusivity: "right now there's CIS male sport and then there's everything else ... there is an argument to be made maybe going forward, we should re-label as 'women and trans hockey'. I think really, it's just non-CIS male hockey”. While the American NWHL has been equally accepting of Harrison Browne, a transgender male hockey player, (Rutherford, n.d.; Hutchins, 2017b), to say that women's hockey has always been an inclusive, welcoming space is inaccurate.

While there has been growing academic interest in transgender lived experiences and sport (Caudwell, 2014; Lucas-Carr \& Krane, 2011, 2012; Semerjian \& Cohen, 2006; Tagg, 2012; Travers, 2006; Travers \& Deri, 2011) and some indication of a growing inclusion for transgender athletes across all sport (Hargreaves \& Anderson, 2014), there has been less research focusing specifically on transgender players in women's hockey. In 2008, Cohen and Semerjian interrogated the banning of a "transsexual male-to-female (MTF) athlete" (p. 133) from USA Hockey. They conducted four in-depth interviews with the athlete who was undergoing hormone therapy and had begun, but not completed, surgical procedures. They concluded that the hypermasculine environment of hockey, even women's hockey, relies on a rigidity of gender difference, a "belief in two distinct, unchanging and immutable sexes" (p. 134) that limits opportunities for transgender players. In "Angela's” case, despite having testimony from three doctors advising she be considered female, and active negotiations over her gender status, when asked to present her birth certificate at the National Championship Tournament, she was disqualified "because her sex was deemed questionable" (p. 137). 
In "Angela's" case, the discrimination was institutional: neither her teammates nor coaching staff registered concern over her gender status; instead, they recognized the ban as "similar to the discrimination they faced in finding available ice times for practice and access to men's leagues" (Cohen \& Semerjian, 2008, p. 137). Despite the support of players and coaches, it was the "hegemonic male authority" of USA Hockey that had the "power to decide what it means to be male or female, and hence assign her an identification" (p. 138). I argue that the inclusion of transgender players in the CWHL and NWHL can be best understood by the autonomy of the leagues. Despite individual team associations between the CWHL and NWHL and the NHL (see Introduction), the CWHL and NWHL were/are stand-alone organizations and as a result, not answerable to any "hegemonic male authority" of an umbrella league. This is a key distinction given the ongoing speculation that the future of the professional women's game lies with the NHL (Wharnsby, 2019). Can the inclusiveness of women's hockey, or "non-CISmale" hockey continue under the auspice of the CIS-male hockey league, the NHL? If women's hockey is a kind of gender catch-all, does this specifically re-enforce a gender binary that reifies the "hegemonic male authority" of the men's game?

Sport relies structurally on a gender binary that continually enforces, not just difference in biology, but difference of value in measurements of strength, speed, aggression and agility that find "non-CIS male" athletes wanting (Theberge, 1998; Messner, 1988 and 2010; Willis, 1982). Kane (1995) argues that feminist critiques of sport need to go beyond proposed resolutions that reinforce biological determinism. Rather than fighting for more visibility and more legitimacy for sports traditionally defined as feminine, academics must focus on ways women have infiltrated sports associated with men. Women represented as athletic, aggressive and confrontational, characteristics traditionally associated with hegemonic masculinity, upends 
the biological determinism of sport and makes visible the structural hierarchy that defines sport as men's natural domain. The demand here is for research that can "provide empirical evidence that many women can outperform many men ... and also that they can possess physical attributes such as strength and speed in greater capacities than do many men (Kane, 1995, p. 197) in order to upend the intrinsic gendered hierarchy of sport. The foundation of this approach rejects sport as necessarily segregated by gender and imagines it instead as a continuum where women can outperform men as a way to counter perceptions that athletic performance is naturally and intrinsically associated with certain gendered (male) traits.

\section{Affect and Aggression}

Theberge's (1998) exploration of women's hockey as a sport continuum provides a valuable theoretical lens through which to examine not just the possibility of a merged women's league under the NHL, but also focus group participants who position this as the best-case solution for the sustainable health of professional women's hockey. Her ethnographic study centres on an elite level women's hockey team, The Blades, and their perspectives on the hockey they play in comparison to the men's, noting that most players' early experiences were in gender-integrated teams only moving to girls' and women's teams as they entered their teen years. The issue of gender segregation in sport has been a source of great debate in academic and activist circles, with some (Tamburrini \& Tännsjö, 2005; Foddy \& Savulescu, 2011; Love \& Kelly, 2011) advocating non-gendered competitions at elite level events while others (Travers 2008; McDonagh and Pappano 2007) argue for maintaining women-only sporting events as a way to protect their hard-won advances. 
Much as focus group participants in this study articulated, Blades players describe the difference in women's hockey in terms of levels of physical violence associated with bodychecking, almost as a gateway to on-ice fist fights, suggesting that they play instead by “brains over brawn” (Theberge, 1998, p. 194). Yet, some players who participated in genderintegrated teams of a like or higher level, describe a willingness and ability to engage in aggressive bodychecking and also an appreciation for the improvement of their own game play. For these players, bodychecking is an integrated material component of the sport for which they have received training and wield with practice. Still, International Ice Hockey Federation (IIHF) regulations identify bodychecking in women's hockey as a penalty infraction. Weaving and Roberts (2012) argue the bodychecking ban contributes to the ongoing marginalization of the women's game, re-enforces traditional stereotypes of female athletes as less capable of physical aggression and denies players' "bodily agency" (p. 470). They suggest the prohibition of bodychecking is more than concern over threats of physical injury; it is instead based on fears of a general increase in the violence of the women's game, even if a linear association between the two is misleading and "rooted in paternalism" (p. 474). I go a step further to argue the distinction is also affective between the physical aggression of bodychecking and the emotional aggression (transgression) of on-ice fighting. Not only does the ban on bodychecking re-enforce the "incompatibility between the female athlete and the (ideologically) ideal woman... which does not fit with the concept of women using their bodies in a forceful and dominant manner" (Weaving \& Roberts, 2010, p. 476), it also negates the risk of over-emotionalism. This is an important distinction for two reasons: 1) female athletes are habitually punished for affective aggression and 2) sport media's reliance on affect to establish audience engagement. 
One example of an athlete punished for affective aggression was discussed in the focus groups. At the conclusion of the 2018 Olympic women's hockey gold medal game when the losing Canadian teamed lined up to receive their second place silver medals. Defence player Jocelyne Larocque immediately removed her silver medal, clutching it instead in her hands while the winning American team's anthem played. Before she made it back to the locker room, an official informed her that she was obligated to wear the medal (Hoffman \& Meixler, 2018). She received intensive backlash from some press and fans on social media "suggesting she had shown 'no class' and had acted in an unsportsmanlike, and even un-Canadian way. 'As a Canadian I am embarrassed,' added one Twitter user" (Belam, 2018, $9^{\text {th }}$ para.). Larocque issued a public apology, explaining her commitment to the games and the heightened emotions from playing do not excuse her failure to act as a good role model (Longley, 2018b).

Most focus group participants were sympathetic to Larocque, disappointed with the action but understood it was the emotional investment in the game that led her to remove the medal. Some participants explained that Larocque's actions allowed the audience to make an emotional connection with her; her fallibility humanized her. Many were frustrated by what they considered to be an unreasonably intense furor over Larocque's action, particularly compared to the more muted reaction two months earlier when Lias Andersson, a Swedish player at the World Junior Hockey Championships, not only removed his silver medal, but also tossed it into the stands (Chidley-Hill, 2018). Whereas Larocque immediately received a stern lecture from International Ice Hockey Federation (IIHF) officials about the consequences of violating medal wearing regulations (Robertson, 2018), Andersson's actions brought him a four game suspension, although this was announced months after the incident and after this research was 
conducted (Steiss, 2018). Participants expressed their frustration at what they perceived as a detrimental double-standard for women hockey players:

I thought it was really unfair that they didn't focus on the women winning the silver. They focused on Jocelyne's disappointment and the fact that she took her medal off from around her neck and then she apologized and put it on after. But I thought that the kind of attention that women got around that was not particularly positive. And for women's hockey, I would hope that they would focus on things that are positive, positive aspects of the game. Not, you know, disappointed player taking the medal off from around her neck for five minutes. (D-4)

A few participants critiqued Larocque's actions for being detrimental to the game and her effectiveness as a role model:

I was born and raised in a military family, surrounded by this sense of duty. So when that happened, I saw that as completely unsportsmanlike. I was very upset. It's disrespectful and I think that as a woman on the world stage and we're trying to promote women's hockey, to pull a move like that was just kind of putting a negative connotation around it. $(\mathrm{B}-2)$

Jocelyne Larocque's decision to remove her silver medal was interpreted by some reporters (Reslen, 2018; Young, 2018) and focus group participants as an act of aggressive confrontation, "unsportsmanlike and disrespectful", rather than the predictable result of the necessary emotional investment in the outcome of an important game.

When Serena Williams breaks her tennis racket and calls out a chair umpire, as she did at the 2018 US Open (Kessel, 2018), this is not seen as evidence of her passion and commitment to the game; instead commentators are quick to characterize it as a "meltdown" (Armour, 2018; 
Haring, 2018), as a breach of normalized gender and racial expression. Positioning aggression in the gendered athlete's body as transgression fails to recognize the ubiquitous structural requirements in sport that demand confrontation, assertion and aggression, and feeds "the habit of connecting active emotions exclusively with the male body and masculine being" (Thing, 2001, p. 276). The tension between "safer and cleaner" women's hockey and a socially constructed gender binary that may allow women to be stronger and faster but denies them the space for physical aggression for the risk it will lead to emotional aggression effectively creates a double bind (Bateson, 1972), an "unresolvable sequence of expectations" (p. 156) for female (and other non-hegemonically white male) athletes that limit participatory possibilities and even threaten health and well-being (Burrow, 2016).

The transgressive association of the female athlete and aggression is no accident. Duncan (2006) identifies emotion as one of ten categories of sport media discursive strategies that frame women's sport in an oppositional gender binary. Her findings indicate that women were most often associated with the emotion of grief (from loss) that turned them "into serious moral people and made them more cooperative and team-or community-minded ... resources for good works, in addition to a higher level of play" (p. 258), particularly when compared to men's passion as the fire driving them to win. Journalists commonly point to the stress women suffer in intense competition due to their "emotional instability" (Duncan, 2006, p. 259). The affective association between female athletes and grief is repeated across all sport.

Ahmed (2004) argues affect or emotion is neither an individual linear biological process nor psychological state but instead is produced in cultural contexts in which identity is performed within specific power structures. "Emotions shape the very surfaces of bodies, which take shape through the repetition of actions over time" (p. 4). The repetition of affect creates a "stickiness" 
so that certain emotions adhere to certain bodies alongside or even before cognitive awareness. By this, we can envision Duncan's observation of grief as affect that sticks to female athletes' bodies through repetition so that alternative affective expression, such as anger and frustration, become discursively transformed into “emotional meltdowns". I argue that in women's hockey, there is a different affective association at play. Rather than grief or "emotional meltdowns", women's hockey players are, as discussed, commonly (repeatedly) represented as "pure", virtuous representatives of a mythical game. On the ice, with faces hidden and bodies restricted in padding, jerseys and helmets, denied and punished for affective excess (bodychecking or lossinspired fury), and still morally "pure", female hockey players are instead affect-less. Can bodychecking allow active emotions to be felt by (and to stick to) the players and through them to the audience?

Weaving and Roberts (2012) argue in favour of bodychecking in women's hockey. Others (Donaldson, Asbridge \& Cusimano, 2013) argue the increased risk of physical injury means that bodychecking has no place in any hockey, particularly in youth hockey (Emery et al, 2010; Lee, 2019; Macpherson, Rothman \& Howard, 2006). Theberge (2000) observes that despite its disallowance, women players engage in bodychecking and other physically aggressive activity in direct contrast to "the rhetoric that portrays women's hockey as 'different' and 'the way hockey is meant to be played"' (p. 126). In her analysis of the media coverage of the 2006 Women's Olympic Ice Hockey Tournament, Poniatowski (2011) identified how commentators pointed to the illegality of bodychecking to construct players in a way that "serves to support stereotypes of femininity - frailty, weakness, passivity and inferiority” (p. 44). Within the parameters of this study, it is important to position bodychecking as a mediatized event, loaded with affective weight, and interrogate how it functions within the flow of affect from, to and 
between the viewer and sport media. Rather than looking at what bodychecking in televised hockey is, I argue the focus must be on what bodychecking does and the role it plays in affective circulation.

While Kavka's (2008) work is primarily in reality television, her theorizing of affect circulation between television, text and audience holds equal value in sport media, two genres that share many of the same productions techniques and affective strategies as discussed below. Kavka turns to affect theory to show how television in general and reality television in particular, works at the level of affect or feeling rather than merely cognition to establish an immediate unmediated intimacy between text and viewer, as though erasing the technology that connects the two. Referring to Grossberg's (1997) work on the wild realism of media texts, Kavka suggests consumption occurs on a variety of planes from the cerebral to the corporeal, that there is "excessive material" (Kavka, 2008, p. 26) in television texts that matters specifically within the zone of affect. Kavka uses the word "matters" here to serve a dual purpose. On the one hand, she sees the affective economy of television as an important site to study excess of meaningeffects, an addendum or alternative to structures of signification, the reading of signified and signifier to understand ideological effects and defects. This is a way of studying television that goes beyond the rational, the cognitive, the representative and the ideological.

Affect also "matters" in terms of materialism, matter in terms of physiology. Kavka argues that reality television in particular engages the viewer in emotional excess in a way other genres don't. The "affective call" is so effective not only because it is experienced by supposedly "real people" but also because it is based on identification with the affective situation itself. This is not imitation or mimicry or simple vicarious joy at the suffering of others but an instinctive understanding of the intensity of feeling on the screen even if the viewer has never expressed it 
or embodied it in exactly the same way (functioning even in marketing as in the traditional "thrill of victory, agony of defeat" from the Wide World of Sports, for example). Kavka calls this affective reality from the screen - something viewers are given to feel rather than necessarily know. Viewers not only identify with the affective situation, their feelings are engaged and amplified and made all the more real because they are shared with the others seen on the screen. This hyper-familiarity not only resonates because viewers identify with the affective situation in the process of resonance or identification, they create an affective reality. Kavka describes a comfort in this shared affective space that occurs at the interface or cusp of viewer and program. Affect is not a solid, immutable state associated or positioned with one body but instead is “dynamic, transportable, transmittable and mobile... unconsciously incursive, creeping or flooding situations with a 'mood' or tone that comes from or 'properly' belongs elsewhere. In its very transportable, dynamic, un or pre-fixable nature, affect has a productive amorphousness" (author's italics, Kavka, 2008, p. 31), moving within and between people and even within and between objects. Kavka is particularly concerned with how affect moves within and between the TV screen, forming what she calls the "cusp" or "point of emergence" for particular material forms the feeling may inhabit.

Kavka suggests that affect can come from a relationship with technology. She argues that mediation is already central to affect in cusp formation or as intermediation; in a technological milieu, affect functions as a "hard-wired circuit" linking bodies, psyches and the world. So, affect that is produced at the site of the cusp formation, even if that site is a piece of technology like a TV screen, that affect is real. Viewers see faces in close-up and recognize the affective state - the recognition is an exercise in affective transmission. TV is uniquely structured to amplify affect and connect real people on one side of the screen with, as Kavka suggests, real 
people on the other side of the screen.

Kavka argues that reality television is a genre specifically suited to the circulation of affect. How does affect circulate in sport media? Kennedy and Hills (2009) describe sport as a "highly physical experience: playing sport is an embodied activity and it arouses embodied responses in its spectators ... a bodily response beyond meaning” (p. 25). Sport media, like reality television, transmits affect to spark audience response, caught somewhere between cognition and emotion, an internal, almost instinctual knee-jerk reaction to the moment, in the moment, a "bodily response beyond meaning" (p. 25). In an ethnographic study of gendered emotional engagement with sport, Gantz and Wenner (1991) note that the differences between male and female viewers' emotional response was less dramatic than expected although certainly evident. They found men's response to sport was significantly heightened in emotion in comparison to women's; men will suffer from withdrawal pains if sport is removed from the television; and men have more willingness to retain their emotional state after the game has ended. The study closes with an acknowledgement that social expectations are undergoing dramatic change; 28 years later, gendered emotional response to sport media requires further research.

When asked to choose one or more feelings they had watching the national team play hockey during the Olympics, online survey respondents overwhelmingly indicated feeling "proud" (81.3\%) and said they felt "excited" (78.0\%) with negligible amounts for the other choices. The $10.8 \%$ who chose to write in an alternative articulated a number of feelings including "inspired" (25.0\%), "patriotic" (18.3\%), "interested" (13.3\%), "impressed" (11.7\%), "nervous" (10\%), "empowered" (8.3\%) and "happy $(8.3 \%)$. The intention was to provide an array of "feelings" for respondents to choose that ranged between affect as instinctual feeling and 
emotion as feeling attached to or associated with object and body as discussed above. Of available choices it is interesting that "pride" and "excitement" were nearly equally chosen. I argue that "excitement" represents affect as per Kennedy and Hills (2009) and Kavka (2008), instinctual and unformed, whereas "pride" is an emotional response when the affect "excitement" interacts with the cognitive process that is nationalism. Of the feelings respondents defined for themselves, the ones closely aligned with affect in terms of instinct, "nervous" (10\%) and "happy" (8.3\%), were significantly lower ranked than the $3^{\text {rd }}$ choice "interested" $(13.3 \%)$ which suggests a more cognitive processing. My argument from these findings is that audience's affective investment in women's hockey during the Olympics, the excitement the respondents feel, is closely related to and even superseded by that specific sport spectacle and its expression of national pride. As per Ahmed, national pride is the emotion that sticks to women's hockey through a quadrennial repetition and becomes the only acceptable affective association with playing and watching women transgress the boundaries of hegemonic femininity. In the absence of national pride, outside the Olympics or other nation based mega-sport events, and as above, drenched in moral virtue, professional women hockey is affectively evacuated.

While survey respondents were not asked to describe their feelings watching professional women's hockey, two questions do provide some interesting insight into affective investment. When asked why women should play professional hockey, $77.8 \%$ wrote answers indicating that it would lead to gender equity while $26.4 \%$ referenced the quality of the women's game play; yet, when asked why people should watch women play hockey, the numbers were reversed with $76.2 \%$ of respondents referencing the quality of the game and $26.8 \%$ of respondents referencing its importance in terms of gender equity. In an open text analysis of the answers from respondents who referenced the quality of the game, $60 \%$ described the quality of the game in 
cognitive terms ("skill”) while 38\% used affective language ("exciting", "fun") and $4 \%$ used both cognitive and affective language. Taken as a whole, of the 558 respondents who agreed that people should watch women's hockey, only 157 (28.1\%) indicated affect as the reason.

Focus group participants were asked about their emotional engagement with women's hockey at both the Olympics and in the professional leagues. Echoing survey respondents, participants indicated feeling a sense of national pride while watching the Canadian national women's hockey team play at the 2018 Olympics and a sense of excitement as they played against their long-standing rival American team. Some participants indicated the feeling of pride was heightened when cheering on the women's team in particular, explaining that the women were succeeding where the men couldn't. D-2 describes her emotional engagement with hockey as a sequence of layered affective intensities:

I feel it strongly when women are playing because this is my pride and this country, this is my women representing my country... watching Montreal Canadiens, I've been watching them since I was born, and it's not the same kind of emotion that goes in. Like, yeah, if they win the Stanley cup, I'll be happy. But I'm won't have that sense of pride that I do when a Canadian team wins the gold or silver. Maybe that's why so many people get engaged into hockey during the Olympics, because it's a question of our country, right? They were representing us.

Other participants talk about finding emotional attachment to women's hockey through stories. One example is the story of the standing ovation for the German goaltender discussed in the previous chapter; two participants in two different groups referred to this story as heart-warming and touching that left them tearful and proud.

In contrast to the cognitive expressions for gender equity in the online survey, focus 
group participants describe feeling emotion attached to the quest for gender equity: "I just get so emotionally involved because you're seeing women do something that's, you know, always been such a male-dominated environment and maybe that's it. Maybe that's why it's so near and dear to our hearts" (D-1). And yet, the open text responses to the survey question coded as equity discussed above, did not include affective language. Focus group participants who played hockey used affective terms to describe their viewing experience: "I feel excitement, but when I watched women's hockey, I feel energy. It's almost like I can relate way better to the women and I feel motivated and driven and like that could be me out there" (B-2). Others described a physical response to the media text, an instinctual reaction to the physicality of the players, the speed of the game, the rush of adrenalin as well as the more cognitive responses of appreciating, learning from and identifying with the players. A-2 articulated this as a feeling based on intimacy and familiarity:

I recognize myself in that game over and over. The skills, the commitment it takes to build the skills, the team play, the physical awareness of yourself and others on the ice. And just the joy, my God, scoring a goal or not scoring a goal stopping a goal. And you're happy.

For E-5 who does not play hockey, emotional response to the professional women's game comes less from the game play than from a connection to the players.

when I watch broadcasts like hometown hockey and you watch the Olympics, the broadcasters and back to $\mathrm{CBC}$ do a really good job talking about the stories behind the athletes. That kind of stuff matters. And I know it takes a lot of time and effort for the broadcasters a lot of work, but I hope they know that it, I feel like it does work.

For focus participants, the connection to professional women's hockey must be built on these 
stories. I argue this is less of a cognitive desire for information or stats, than it is to establish what Kavka (2008) refers to as intimacy between viewer and (reality) television text. This intimacy demands the invisibility of the technology, so that the technology, or screen or cusp becomes affectively entangled and obscured. Reality matters because the "real people" on the screen are defined as such, "real" athletes like the "real" people watching. I argue that in sport, affective circulation, particularly in terms of heightened "active" affects of aggression, assertion and confrontation, depends on the same familiarity, not with recognition of the viewer to the athlete (which would discount non-male fans of men's sport) but with the affect flowing from the (appropriately male) athlete's body. The familiarity of the associated affect and body allows for the affect to circulate freely whereas the transgression of the affect with the female athlete's body becomes noticeably mediated. The exception of course can be found in events such as the Olympics, when gender, class and race identities become subsumed in the dominating representation of nationalism.

When focus group participants spoke specifically about professional women's hockey, the affective investment was noticeably reduced, perhaps not surprising given that not all had seen a CWHL game. Support for the growth of professional women's hockey was framed as a feminist activity, a matter of gender equity, of political activism and this in turn becomes paradoxically associated with hegemonically feminine virtue. Unlike the emotional terms D-1 used above to describe the feeling of equity attained even just for that game moment, many participants statements indicate that equity for professional women's hockey has yet to be achieved, watching women's hockey then takes on a component of moral obligation, the right thing to do. Watching professional women's hockey becomes a commitment to providing role 
models for young girls, for supporting gender equity, for closing the pay gap, for combating hockey's hypermasculinity.

Poniatowski and Hardin (2012) deconstruct the characterization of the role model for women's hockey noting that, unlike previous research, their participants were more likely to refer to a female hockey player as a role model than a man. Despite the seemingly progressive nature of this finding, the authors note a disparity: the role model, either male or female, is seen to be necessary only to the female players. Male hockey players are inherently legitimate, in no need of guidance or support to perform their natural function. Women, on the other hand, as invaders or interlopers in this masculine domain, require both inspiration and an approval that is never quite given (Poniatowski \& Hardin, 2012). (from pg 137). In an interview for an article on the state of women's hockey, advocate Deirdre Norman goes a step further to argue that associating women's hockey with stereotypically feminine traits, such as virtue, purity, and community isn't just an ineffective path to sustainability, it is detrimental:

We need to stop approaching the CWHL with a "bake-sale mentality." The CWHL markets the league as having "good role models for girls," so that younger fans will see that they, too, can be athletic and skilled and still be a girl. The NHL doesn't rest the bulk of its marketing on having good role models, so why does women's hockey? Jocelyne Larocque's decision to remove her medal after it was placed around her neck shows that women don't always conform to stereotypes - but they can still be great athletes. She made a choice in the heat of the moment, one that she would probably take back if she could — but does that make her any less of an outstanding hockey player? Absolutely not. (as cited in Taylor, 2018, 13th para.)

Larocque's transgression risks her suitability as role model and threatens the perceived social 
value of women's hockey. Women as hegemonically feminine role models must also abide by the unwritten constraints of behaviour that dictate women may be emotional, but that emotional expression must be virtuous, innocent and "pure". This is particularly true if the athlete competes in male-coded team sports such as hockey or soccer at the risk of being perceived as masculine. Underlying this is the question of sexual orientation. Historically, social perceptions about female athletes have been tinged with homophobia (Fink, 2012). A physically muscular appearance translates as masculine and puts sexual orientation in question. Even as more female hockey players are coming out (Proteau, 2014) and women's hockey is seen to be genderinclusive, value is still ascribed, even a recuperative value, to the athlete who can be simultaneously strong and feminine, who is then a "culturally acceptable woman" (Krane, 2001, p. 116). This discursive contradiction, that female athletes be strong enough to compete, but not so strong they risk their femininity, is pervasive in sporting cultures. Hegemonic femininity as a social construction in a patriarchal culture defaults to heterosexuality, or as Adrienne Rich (1980) puts it "compulsory heterosexuality." Representations of femininity then must subscribe to its construction: women must be weak, emotional, dependent and follow to gender-appropriate behaviour. Female athletes, particularly female athletes in sports traditionally defined as male, must navigate the expectations of hegemonic femininity and strong bodies. Vikki Krane (2001) notes that any transgression of heteronormative appearance and behaviour has led to heterosexism and homonegativism: "women in sport who do not conform to the feminine ideal and who are perceived as masculine are labeled as lesbian" (p. 119). The consequences of this label include lost revenue and sponsorship, harassment, physical harm and discrimination (Blinde \& Taub, 1992; Griffin, 1998; Krane, 1998; Veri, 1999). Meanwhile, athletes who successfully perform hegemonic femininity can reap rewards. Simply renegotiating gender 
norms to include muscular women as feminine maintains the sexual difference which reestablishes sport's compulsory heterosexuality.

Norman (cited in Taylor, 2018) advocates a distinctive re-framing of the game, not only in the media but also from the players and institutions, that recognizes and rejects the marginalization affirmed by discourses that associate women's hockey with purity, morality, virtue. Consider Nancy Theberge's $(1995,1998,2003)$ ethnographic work on women's hockey that stretches back over twenty years. The discursive framework used by her participants was the same then as it is now: women's hockey is a "pure", morally superior iteration of the game. While participants and respondents express a conceptual commitment to women's hockey, this long established framework is clearly not compelling them to watch. While participants and respondents express a conceptual commitment to women's hockey, this long established framework is clearly not compelling them to watch. In the next chapter, I will explore alternative frameworks that resist discursive hegemonic femininity and consider instead the ways that affect can circulate between professional women's hockey and a mediated audience.

From the participants, what appears to be missing is an affective connection or, as per Kavka, affective circulation between the professional women's hockey media text and viewer through the cusp of the television screen. As mentioned above, Kavka positions her theory of affect and intimacy in reality television suggesting the affective circulation flows freely between the 'real people' on screen and the real people in the audience. Dominguez (2015) identifies a reality television sub-genre, The Real Housewives docusoap franchise, as melodrama, a form that publicizes and makes spectacle of the private and the everyday. Like its fictional counterpart, the soap opera, The Real Housewives features recurring characters in an intimate story space (housewives) that becomes a grand stage for the circulation of a sensational emotionality: 
offering disorienting and anxiety-inducing explosions of affect that are then followed by cathartic discussions of emotion (focused on understanding the previous outbursts), creating for viewers a sense of connection with the program and its cast through their ability to witness these moments of intimate discord, whether between kin or friend. (Dominguez, 2015, p. 159)

Dominguez identifies as trope that moment in every iteration of the franchise when conflict builds to a spectacular release, fights, occasionally physical, erupt between well-coiffed and impeccably made up women. Describing these moments as the predictability of unpredictability, Dominguez borrows the term "money shot", a pornographic reference that refers to male orgasmic ejaculation as the only acceptable proof of real physical arousal (Williams, 1999). This is a well-established strategy to heighten affective intimacy with viewers, particularly in reality television. Bruce (2009) describes the "spectacle of the "reveal" (p. 83) as the affective pinnacle of home improvement reality programs while Grindstaff (2002) refers to the emotional outburst of talk shows like the Jerry Springer Show. However, whereas the home improvement money shot is a temporally limited affect that can include joy, sorrow or rage, in the docusoap serial, the money shot is built around anger and jealousy and is drawn out and built up over multiple episodes, even revisited and, in essence, re-felt, during the ubiquitous reunion shows. Viewers feel intimately connected with the characters through these affective outbursts even though the promise to discharge is never fully achieved and never will be achieved, so they can re-feel the affective state as it is discussed, dismantled and ultimately deferred to the promise of the next episode. The thrill of / anxiety over constant deferral combines with the familiarity of the characters and compels the viewer to keep watching. I argue below that, much like soap opera 
and reality television texts, sport creates and circulates affect as strategy for audience engagement.

\section{Soap Opera and Sport}

Feminist scholars (Ang, 1985; Brunsdon, 2000; Geraghty, 1991; Modleski, 1979) have long sought to understand viewer engagement with a genre that "targets and exploits the female audience, selling them 'images' of themselves that are deeply problematic and indicative of the contemporary epoch of postfeminist media culture" (Cox, 2012, p. vii). They argue that pleasure results from the negotiation with the encoded messages of gender, race and class identity allowing viewers to "critique televisual discourses at the same time that they intimately engage with them" (Mankekar, 1999, p. 14). Drawing similarities between soap opera and sport media has provided a framework for scholars (Gantz, Wang, Paul, \& Potter, 2006; Lavelle, 2015; Whannel, 2002) to examine emotional engagement with two popular "talked about texts" albeit ones firmly "divided along gender lines" (O'Connor \& Boyle, 1993, p. 108). While soap operas feature fictional characters in domestic situations and sport is a non-fiction public event, both texts are formed and manipulated through structured production components in terms of camera angle, reply and music. Even the ubiquitous instant replay of televised sport is repurposed as post commercial break story summary for soap operas and reality television. In this way, the docusoap provides an even more compelling comparative in that the characters, like the athletes, are identified as real, although structured as contrivance, "real people playing themselves" (Hall, 2009, p. 516). O'Connor and Boyle also describe similar viewing practices, sport and soap opera as an everyday, leisurely activity, and the ways emotional identification is constructed from the 
text that relies on and supports characteristics of gender normativity. This pleasure comes to viewers by way of recognition (Ang, 1985).

For O'Connor \& Boyle (1993) the most marked similarity is the "play of emotion and the invocation of a strong emotional response in the viewer" (p. 112), or as Kavka (2008) would have it, the affective circulation. Whereas traditionally feminine characteristics presume unhindered emotionality, the authors suggest that sport provides one of the few arenas where men can indulge in strong emotional expression without censure or threat to masculinity; although, one may also perhaps consider Supreme Court nomination hearings to be a recently added venue for male affective performance or presidential twitter explosions. I suggest that the distinction is even more discrete. Sport may be an acceptable venue for male emotionalism but, as discussed above, it is decidedly hostile to female affective expression. The point may be taken further to argue that the only cultural space afforded to women to express extreme emotionalism is in the domestic sphere of the soap opera or docusoap.

O'Connor and Boyle (1993) argue that the affective circulation in both sport and soap opera (and I would add, reality docusoap), relies on an identification with characters and players, a "process of personalization" (p. 113) that reaches beyond the temporal constraints of the specific media text. Much as viewers watch soap opera characters grow up on screen, recalling storylines and alliances from the past that contextualize the present, sports fans follow players from the minors to the professional, changing teams or positions, wins and losses, injuries and recoveries. Viewers create "prolonged and continuous" relationships with characters through the open text of their personal narratives and the genres. While the end of the soap opera episode promises the upcoming drama, so too does the whistle at the end of the game lead viewers to the re-match next week or next season. Connections are created through personal profiles in pre- 
game spots, profiles in supporting media and the staple of the docusoap, the confessional interview (Coles, 2000).

If indeed sport is a "male soap opera" (Morse, as cited in O'Connor \& Boyle 1993, p. 109) and soap opera provides "pleasures of identification and involvement ... available only to women" (Geraghty, 1991, p. 167), what can be learned about audience emotional engagement, or lack thereof, in professional women's hockey? As Geraghty argues, the soap opera audience has broadened to include men and, as discussed above, women are equally engaged in male sport soap opera, which suggests the issue reaches beyond a gendered audience. Results from this audience study indicate clearly that professional women's hockey as a concept or media text does not affectively engage viewers. Few focus group participants described feeling emotionally connected to the game and players; those that did most frequently were players of women's hockey, knowledgeable about the games, the leagues and the players. Only $7.0 \%$ of online survey respondents could name all the players on the Canadian National women's hockey team, while $69.2 \%$ could name some of the players. Focus group participants were able to identify some Olympic players, mainly higher profile athletes who have also worked in sport media; however, few were able to name specific CWHL players or teams.

Well over half (59.4\%) of online survey respondents indicated lack of information as the reason why they didn’t watch professional women's hockey. Similarly, focus group participants commonly pointed to a lack of publicity. As mentioned in the previous chapter, former CWHL Commissioner Brenda Andress described a strong campaign run by the league and broadcaster leading up to the 2018 Clarkson Cup (personal communication, June 10, 2019). Further to my argument above, publicity about game times and channel is not the main barrier keeping audiences from watching professional women's hockey. What focus group participants describe 
is a lack of emotional connection with the players and the game which is extrapolated into an anticipated lack of affective circulation between viewer and media text. Whereas women's Olympic hockey benefits from the emotionalism of patriotism and the structure of spectacle, professional women's hockey has been denied the opportunity to circulate strong emotional response, instead positioned by proponents, as "pure", "strategic" and "politically empowering", who "respected the players for playing smarter, for being more intelligent" (E-1). The game then is disengaged from the players and any emotional investment viewers may make with them.

\section{Mixed Methods Audience Research}

This research is prompted by a desire to understand why audiences will and won't watch professional women's hockey; or, more specifically, to examine the inconsistency between what audiences say they want and what they do. Given that participants and respondents took the time and made the effort to participate in this research for no compensation, I contend that the audience studied in this research was already interested in women's hockey and interested in supporting this research before it began. And yet, even among this audience of the converted, relatively few watched professional women's hockey with consistency or commitment. This presents a contradiction that requires the rigour of mixed methods to unravel. The quantitative component provided data from a large sample $(n=685)$ that indicated overall trends in how audiences watched, thought and felt about women's hockey. The qualitative component allowed for a deeper interrogation into the reasons and uses audiences have or don't have for women's hockey in a much smaller sample $(n=25)$. Each individual component produced valuable data but on its own provided only limited insight into women's hockey audiences. I argue that the 
mixing of these methods didn't simply allow for more valuable data or even a deeper exploration of the theories I engaged. In this study, the rigour of mixed methods resulted unexpectedly in a validation of the impact on audiences of unaffective media events.

This is important when considering the growth of the audience for women's hockey. Advocates and activists argue the importance for increased audience and media coverage for women's hockey for a number of political reasons including gender inequity, the importance of role model for young girls and the wage gap. At the heart of the struggle lies the physical and emotional constraints of gender normative behaviour that must be challenged. Thing (2001) concludes from her ethnographic study of female athletes and aggression that while there is an element of play-aggression that reproduces hegemonic masculine values in sport, the benefits must be considered. She describes the pleasure and freedom felt by female athletes from the hard physical work of sport, that allows them to know and learn from their bodies and redefine them as "not-weak and not-fragile ...to invert the common-sense belief in the naturalistic female body" (p. 286). From Thing (2001) and Theberge (1997, 1998, 2003), and from focus group participants, the playing of hockey elicits intensely active affects. The circulation of these affects depends on and ultimately contributes to the destabilization of the biologically determined, feeling, gendered body. 


\section{Chapter 6 Conclusions}

In July 2018, I presented the preliminary findings of this research at the annual Hockey Conference at the University of Alberta. Since its inauguration in 2001 at Saint Mary's University in Halifax, the conference has drawn together academics, athletes, artists and activists from around the world to present and discuss the many "historical, empirical, conceptual, and artistic approaches to the sport" (www.thehockeyconference.ca). In conference materials, amongst the location and accommodation details, was a reminder for me to pack my skates for the conference's annual game of shinny. Presumably, hockey scholars are hockey lovers and any gathering is an opportunity to shoot the breeze — and the puck.

Unlike many of the other conference attendees, I do not play hockey. My interest in hockey is in how it functions as a cultural discourse, how it works to reaffirm normative gender ideologies, and how it so stubbornly excludes women. In many ways, my interest in sport in general results from a desire to understand my own childhood as a reluctant athlete. Perhaps it was being bigger than everyone else, taller certainly, including most of the boys. Already my physical representation of hegemonic femininity had some transgressions; sport, I feared, might compromise this further. Later in life, when I no longer cared about such things, I discovered for myself the connection between physical and mental health and witnessed the camaraderie and community that can come from team play. My work in sports and hockey is as much for other girls who may feel alienated or excluded from the world of sport.

Despite my romanticized view of sport and hockey and its benefits as such, it is worth noting that many scholars and athletes are now problematizing sport, specifically examining the risks posed to children and youth in sports, including sport specialization and its necessarily intensive training too early in life (Feeley, Agel \& LaPrade, 2016; Moseid, Myklebust, Fagerland 
\& Bahr, 2019). Parissa Safai (2003) investigates risks of injury in sports and the culture that normalizes pain for male and female athletes. Even supposedly "progressive" campaigns such as “Throw Like a Girl” (https://www.youtube.com/watch?v=XjJQBjWYDTs) and "This Girl Can” (http://www.thisgirlcan.co.uk) that challenge the hegemonic masculinity of sport by celebrating alternatives to the sexualized and hyperfeminine female athlete have been critiqued by scholars (Fullagar \& Francombe-Webb, 2015) for the use of "Girl" in "This Girl Can" to represent women and the infantilizing and fetishizing of female flesh for the male gaze, and control over that body. Dawn Heinecken (2015) critiques the seemingly progressive fictional narratives of the empowered "tough" girl athlete who participates in all sports without restriction, develops close relationships with teammates and is unapologetically competitive. While the character presents a challenge to hegemonic feminine identity, Heinecken argues these "transgressions" are welcome only in the sporting environment, noting the underlying message that girls should "should keep their on-field identities separate from their real lives" (p. 28). Between its entrenched masculine culture, its complete corporatization and its "insidious" effectiveness at perpetuating ideological norms, sport, particularly sport media discourses must continue to be examined critically by scholars, activists and athletes.

Still, the intention of my research study, to learn about women's hockey audiences and why they will or won't watch professional women's hockey, is inspired by the voices of athletes and activists who demand that women be allowed the opportunity to play, and make a living from, professional hockey. This follows Itani's (2015) directive to make visible the experiences of athletes to help "deconstruct the normative and normalizing discourses of women and female sporting masculinities" (p. 265) and adds to it by providing a voice for the otherwise silent hockey audience to be heard. Conceptualizing the audience for women's hockey is important, 
especially an audience for professional women's hockey; as has been recently demonstrated, without an audience there simply is no professional women's hockey. Activists argue that building a broadcast audience for women's hockey begins with increasing the number of spectators to lives games (Andress, personal communication, June, 10 2019); certainly a full arena loans a sense of excitement and interest to the media text. Focus group participants and sports reporters argue for increased broadcaster investment or inclusion under the umbrella of the media juggernaut that is the National Hockey League (Rutherford, 2019). The results of this study indicate the issue with women's hockey audience growth is less to do with media coverage and financial investment than with audience affective engagement.

This chapter summarizes the findings of this mixed method study, acknowledges its limitations and provides recommendations for further research. I summarize with a statement on how the conclusions of this study may inform strategies to grow the audience for professional women's hockey.

\section{The Findings}

The findings of this mixed method study show that while audiences want women to play professional hockey and want an audience to watch women play hockey, they generally don't watch it themselves. To excavate the paradox between what audiences say they want and what they do, I turned to affect theory to both establish mediated sport as a particularly effective circulator of affect, much like soap operas and reality television; and to establish the affective connection audiences will and won't make with women's hockey during the Olympics and in the professional leagues. Survey respondents and focus group participants indicated a lack of media 
coverage for the professional women's game keeps them from watching. This is mirrored in participant statements desiring personal connections with the players, their stories and those storied games. I contend that lack of information and connection is instead a desire for familiarity with the characters and settings and an intimacy with the television text that, as per Kavka (2008), allows circulation of affect.

This is not to claim that the lack of promotion, media coverage and marketing doesn't plague women's hockey, or indeed all women's sport (Cooky et al., 2013; Cooky et al., 2015) struggling to find broadcast audiences. Given that the sample population was from an already established women's hockey audience, an audience who cared enough about women's hockey to take the time first to do a survey and then even more time to participant in a focus group, an audience who expressed their commitment to women's hockey as an idea, that this audience of the converted, per se, still does not watch professional women's hockey indicates the issue is more than lack of information. Instead, I contend that audiences are seeking an affective engagement with professional women's hockey that has been rendered affect-less or affectdepleted as a result of three discursive strategies. First, a result of its repetitive quadrennial association with the Olympics, women's hockey is most affectively associated or sticky with national pride; in this case, patriotism functions to solve the gender transgression of women playing the hypermasculine sport of hockey. Secondly, the characterization of professional women's hockey as the "pure" representation of the game manifests the myth of Canadian hockey as a non-commercialized sport. Women's hockey then acts as a nostalgic placeholder devoid of context that ultimately serves to limit affective circulation. Whatever affect there is from nostalgia, it becomes attached or stuck, not to women's hockey, but to the myth they represent. The framing of women's hockey as "pure" because it has resisted the lure of crass 
commercialism functions as a double bind to ensure the financial sustainability of professional women's hockey remains at risk.

Lastly, forbidding bodychecking and other body contact play even as it occurs on the ice is an exercise in institutional gaslighting. While being mindful of research exposing the pervasive discourse of athlete "toughness" (Messner, 2015; Safai, 2003) and the developing awareness and protocols around concussions (Sanderson, Weathers, Snedaker, \& Gramlich, 2017), I argue the aggression of hockey play, of any sport for that matter, carries affective weight and yet female athletes who perform aggression or other active affects are routinely punished for transgressing the structures of hegemonic femininity (Allen \& Frisby, 2017). In women's hockey, all affect, with the exception of national pride, is limited or denied as a way to solve the risk to hegemonic femininity in the hypermasculinized arena of hockey.

The use of mixed methods in this study has provided valuable insight into the unaffected audience for women's hockey. The value of this insight must also be considered as a finding of the study and as evidence of the fruitful possibilities of convergent audience reception studies as called for by Livingstone (2019) and others (Gunter, 2000; Morley, 2006; Schrøder, 2013). The rigour required of mixed methods in data gathering and analysis allows for more than deeper or richer results from each component. Following grounded theory, the mixing of methods, specifically the constant comparison of quantitative data and qualitative data allowed findings and theories to emerge from the data. Mixed methods in this study resulted in an unexpected result: a quantifiably validated assessment of the impact of unaffective media events on the audience. This indicates benefits for the future of audience research following mixed methodology and informed by grounded theory. 


\section{Limitations}

One of the unexpected strengths of this study resulted from the targeted population from which the sample was drawn. Knowing that participants and respondents were already predisposed to have an interest in women's hockey and yet still didn't consistently or with commitment watch professional women play indicated the issue was more than mere lack of knowledge or exposure. And still, it is important to acknowledge recruitment as one of the limitations. While snowball sampling is academically acceptable, relying on personal networks led to a demographic homogeneity that may or may not reflect the broader audience for women's hockey. As well, snowball sampling and other non-probability sampling that rely on volunteers to self-select for participation means there is no reliable sample size to calculate a margin of error indicating possible difference between results and the "real" population the sample represents. The smaller the margin of error, the more the results are statistically reliable. While non-probability sampling such as snowball are academically acceptable, drawing a sample from a defined population such as a community, regional or even national hockey league or association would have provided more statistically reliable quantitative results.

\section{Recommendations Future Research}

Professional women's hockey is currently facing a crisis. Whatever specific gains in terms of audience growth and financial stability made over the years of the CWHL have been lost with the league folding. While optimistic voices prophesy a re-birth, possibly as one league, possibly under the moneyed auspices of the National Hockey League, the results of this study 
suggest that unless professional women's hockey undergoes a drastic re-framing, audiences will remain unaffected and non-spectators. Certainly, the established meta-narrative of professional women's hockey inspires little affective engagement. Media stories that focus on the infighting between two leagues (Noel, 2015), the difficulties paying the players (Berkman, 2017) or even keeping them well equipped (Stone, 2015), function to redirect audience attention away from the game. Even before the Canadian league folded, the perception that professional women's hockey was in a constant state of crisis was already established.

If, as Ahmed argues, emotions "shape the very surfaces of bodies, which take shape through the repetition of actions over time" (Ahmed, 2004, p. 4), changing the emotions, or intensity of emotions, currently stuck to women's hockey is also possible through repetition. One strategy will be the resolution of the double bind that demands women play an aggressive sport without allowing them to be bodily aggressive. Another example may be found in the newest of a seven-season series from the Aboriginal People's Television Network. The 2019 season of Hit The Ice (https://aptn.ca/hittheice) follows a group of young aboriginal women at a two-week training camp run by Olympians and professionals there to recruit players for university teams. In this emotionally powerful series, young players are presented as fully realized characters, defined but not limited by hockey, unapologetically competitive and aggressive and focused on the game. Production techniques common to the reality docusoap including confessional interviews combine with active and aggressive game play allow affect circulation between the characters and the audience through the television screen. The representation of aboriginal female players is also an important step towards battling the perception of racial and class homogeneity in the women's game. 
Women's hockey must also be interrogated as a Western-defined sporting activity with little racial diversity and high financial barriers. With the recent development of women's hockey in China and the success of the Finnish team in the recent Women's World Cup of Hockey, there is a strong demand for academic research that considers women's hockey from a transnational perspective. Wrapped as it is nationally in the construction of the Canadian male identity, how does hockey function in other cultures, as a particularly physical representation of gender characteristics and "the historical association between gender physicality and power" (Theberge, 1997, p. 70)?

Many scholars have identified sport in general and hockey in particular to have great representational power to define gender. As a result, the inclusivity of women's hockey where non-gender binary players have been accepted and embraced is fertile ground for further academic exploration of sport as an activity and as a media discourse that can function to challenge sexual difference. Given that many young hockey players start their experience in a non-gendered league and as one focus group participant observed "the women's game is becoming more like the men's game, but more interestingly the men's game is becoming more like the women's game" (B-1), scholarly research must undertake an examination of the future of professional hockey and its possibility as a gender convergent sport.

While there has been increasing academic interest in the female sport fan experience, the lack of research into the audience for women's sport must be addressed, particularly the female viewer/spectator for women's sport. Additionally, given that the representation of men who participated in this study was small, but not insignificant $(19.3 \%$ of survey respondents and $20 \%$ of focus group participants), I argue that the male viewer of female sport is worthy of academic attention. Research must include examinations of recent campaigns attempting to destabilizing 
sport as a masculine domain such as Nike's "Dream Crazier"

(https://www.youtube.com/watch?v=whpJ19RJ4JY), “Throw Like a Girl”

(https://www.youtube.com/watch?v=XjJQBjWYDTs) and "This Girl Can”

(http://www.thisgirlcan.co.uk) and the effectiveness of female athlete role models, particularly

considering research showing that girls are dropping out of sport in large numbers (Crane \&

Temple, 2015; Plaza \& Boiché, 2017).

Lastly, the history of professional women's hockey requires further academic research.

The story, fraught with conflict, infighting and secrets, is about different groups of women who want the same thing, to be able to play professional hockey. Systemic discrimination and the limitations of hegemonic gender norms inhibit this. Rather than working together to overcome the common foe, as it were, the groups of women instead battle each other over what amounts to the crumbs allowed them in a patriarchal society. Future academic research must expose how patriarchal structures depend on a divide and conquer strategy that ensures gendered inequity remains intact.

\section{Growing The Audience For Professional Women's Hockey}

In women's hockey, the children are indeed the future. Studies have shown the number of young girls signing up for recreational and community leagues in Canada is growing (Murphy, 2018). The CWHL had already identified these players as their future audience by offering free game tickets to jersey wearing girls. Whatever the future iteration of professional women's hockey, this association must continue to be exploited. Young girls don't just take themselves to games, they come with economically valuable parents and family members who buy tickets, 
snacks and merchandise. Hockey associations at the local, regional and national level should emphasize the connection between spectating professional women's game, watching broadcasts of professional women's hockey with establishing a possible future or even a dream for hockey playing girls.

Certainly growing an audience for women's hockey must include women viewers. Women watch men's hockey for a number of reasons from the feeling of nationalist pride during a mega-event such as the Olympics, to team-based loyalty, to the affective excitement of fast and aggressive play. While female participants in this study articulate these same objectives watching women play hockey, particularly national pride during the Olympics, they were also apt to describe watching women's hockey as a statement of solidarity, a way of supporting the fight for gender equity. Equally important is the development of male audiences for women's hockey. The participants and respondents who identified as male represented a small but enthusiastic minority. Much as men's hockey has identified the need for audience expansion to include all genders, so too must women's hockey. The majority of participants and respondents used terminology that imbued women's hockey with purity and virtue and ascribed value to the game and players as role model for young girls. I echo Deirdre Norman's recommendations to reject characterizations of the women's game as "pure" as a postfeminist discursive strategy that functions to uphold the limitations of hegemonic femininity and a double bind that keeps professional women's hockey from a financially stable future (Taylor, 2018).

I will close by referencing an article written by Scott Stinson (2018) for the National Post charting the ascension of men's minor hockey from "small town curiosity to must see TV" (1st para.). The World Junior Ice Hockey Championships have been broadcast on TSN since 1991. Today, the tournament's annual final scheduled just after Christmas is watched by millions of 
people in Canada and around the world (Stinson, 2018). Stinson talks about the camaraderie in the broadcast booth, reporters and cameramen who sacrificed the holidays with their families to capture these teenagers who, like women hockey players, play for the love of the game, rather than immediate commercial stakes. In this case, the purity of the game is the draw, audiences enticed to see these players in their "pure" state — before they become corrupted with million dollar NHL salaries. This is, as they say, 'good hockey'. Audiences are enticed by the temporality, a moment in time that they can refer to well after the player has gone onto NHL fame.

The commitment in the booth to good hockey was soon to be matched by the whole TSN organization:

TSN's influence on the tournament's growth cannot be denied. Consider that it has for a decade now been the host broadcaster for the world juniors, even when they are played across the Atlantic Ocean. In that role, it is intimately involved in all aspects of the tournament, it makes all the decisions about television production, whether in Sweden or the Czech Republic or Canada, and provides the feeds that are used by international broadcasters. Because TSN produces all 31 games, that means those networks can show their home team start to finish. (Stinson, 2018, 17th para.)

Broadcaster commitment certainly ensures that all hockey games are covered. With just four out of 87 games broadcast, professional women's hockey has never received that same level of commitment; in contrast, broadcasting only four games conveys the message that the broadcaster, the expert on all things to do with televised sport, believes the value is not there. The four games instead becomes a gesture or statement for social good. Having the broadcaster "get intimately involved" means not just promotion and marketing on the individual channels but 
working to frame the games as must see TV. The article by Stinson answers the chicken and egg of what comes first: broadcaster investment or the audience. Clearly, with junior men's hockey, the audience resulted from broadcaster investment, not just in the games covered but in the framing of the game as legitimate.

The findings of this study indicate that there is an interested audience for professional women's hockey, a product-in-waiting needing broadcaster investment to complete its construction. How then to compel a broadcaster to make this investment, especially given the industry's changing and challenging economic landscape? One answer may be found in the new season of Battle of the Blades, a reality competition series produced by Insight Productions for Canada's public broadcaster, the Canadian Broadcasting Corporation (CBC). Battle of the Blades pairs a hockey player with a figure skater to train and compete in a figure skating competition. In its four previous seasons from 2009 to 2013, all teams but one have consisted of a male hockey player and a female figure skater. Only once has a women's hockey player competed with a male figure skater; in 2011, Olympian and Toronto Furies player Tessa Bonhomme skated with gold medalist David Pelletier to win the third season (cite). In April 2019, the CBC announced it was responding to audience demands and re-launching the series (Wong, 2019). This season will feature two professional women's hockey players, Natalie Spooner and Amanda Kessel (Canadian Broadcasting Corporation, 2019).

Despite the fact that the women are only two of seven hockey playing competitors, the promotional material for the series prominently features a photograph of an unidentified female hockey player, her long blond ponytail hanging down from underneath her helmet. The promotion is based less on the personality of the skater than on her gender. I contend that this 
promotional material is intended to capitalize on the increasing public awareness of women's professional hockey and also gestures to the women's game as legitimate.

As a public broadcaster, the $\mathrm{CBC}$ is obliged to follow the Broadcasting Act, S.C. 1991, c. 11 that dictates the Canadian broadcasting system should:

through its programming and the employment opportunities arising out of its operations, serve the needs and interests, and reflect the circumstances and aspirations, of Canadian men, women and children, including equal rights, the linguistic duality and multicultural and multiracial nature of Canadian society and the special place of aboriginal peoples within that society. (Canada, 1991, p. 3)

As Phillips (2018) argues, for many decades the CBC constructed a valuable audience for men's hockey, often to the detriment of other alternatives. As a result, half of the population has been consistently and routinely excluded from the cultural discourse of Canada's national sport. I argue that the $\mathrm{CBC}$ has an obligation to right this wrong. I further argue that if there there is a perceived value in promoting a reality series on the backs of professional women hockey players, then there must be value in constructing an audience for the professional women's game.

Whether Battle of the Blades works to establish audience affective engagement with professional women's hockey remains to be seen. Survey respondents and focus group participants certainly called for, not just increased media coverage, but deeper and richer coverage that would allow them to make a connection with the game and the players. Coverage must also include commentary and daily sports reporting that go beyond the "problem with women's hockey" to cover games, to talk about trades and player statistics. Crucially, this coverage must also reject the automatic association between women's hockey and hegemonically feminine virtues that denies the players the opportunity to be competitive, aggressive and 
commercially viable. Broadcasters must frame the women's game, not as "pure" or virtuous, but as good hockey. Institutional regulations must be re-written to resolve the disconnect between allowed and denied physical aggression in a physically aggressive game. Lastly, professional women's hockey must resolve internal differences to present a united organization as one league; the 200 professional women hockey players who have gathered, unionized and initiated job action indicates this may be on the way. 


\title{
Appendices \\ Appendix A - Ryerson Research Ethics Board Approval
}

$7 / 8 / 2019$

Email sent to applicant on January 12, 2018 11:44:45 AM

Ryerson

University

Ryerson Home > Online Ethics Submission and Review System > Protocol View

\section{Email sent to applicant on January 12, 2018 11:44:45 AM}

\author{
From: rebchair@ryerson.ca \\ To: dgall@ryerson.ca, ,jbruce@ryerson.ca \\ Cc: rebchair@ryerson.ca \\ Subject: REB 2017-377 Status \\ Date: January 12, 2018 11:44:45 AM \\ REB 2017-377 \\ Project Title: FROZEN OUT: AUDIENCE, AFFECT \& WOMEN'S HOCKEY
}

Dear Donna Gall,

The Research Ethics Board has completed the review of your submission. Your research project is now approved for a one year period as of Jan 12,2018.The approval letter is attached in Adobe Acrobat (PDF) format.

Congratulations and best of luck with the project.

Please note that this approval is for one year only and will expire on J anuary 12, 2019. Shortly before the expiry date a request to complete an annual report will be automatically sent to you. Completion of the annual report takes only a few minutes, enables the collection of information required by federal guidelines and when processed will allow the protocol to remain active for another year.

Please note that REB approval policies require that you adhere strictly to the protocol as last reviewed by the REB. Any modifications must be approved by the Board before they are implemented. Adverse or unexpected events must be reported to the REB as soon as possible with an indication from the Principal Investigator as to how, in the view of the Principal Investigator, these events affect the continuation of the protocol.

Please quote your REB file number (REB 2017-377) on future correspondence.

If you have any questions regarding your submission or the review process, please do not hesitate to get in touch with the Research Ethics Board (contact information below).

No research involving humans shall begin without the prior approval of the Research Ethics Board.

This is part of the permanent record respecting or associated with a research ethics application submitted to Ryerson University.

NOTE: This email account (rebchair@ryerson.ca) is monitored by multiple individuals. If you wish to contact a specific member of the Research Ethics Board, please do so directly. 


\section{Appendix B - Online Survey}

Women's Hockey Audience Reception Study

Consent to Participate in Research

The Survey

1) Among all the Winter Olympic sports, how important is hockey to you?

( ) Very Important

( ) Somewhat Important

( ) Not very Important

( ) Not at all Important

( ) Don't know

2) During the Winter Olympics, would you rather watch Men's Hockey or Women's Hockey?

( ) I would rather watch Men's Hockey

( ) I would rather watch Women's Hockey

( ) I would watch both equally

( ) I would watch neither

( ) Don't know

3) Have you, or are you planning to, watch the Canadian National Women's Hockey Team play during the 2018 Winter Olympics?

( ) Yes

( ) No

( ) Maybe

( ) Don't know

4) How will you watch the Canadian National Women's Hockey Team play during the Olympics? (choose one or as many as applicable)

[ ] Television

[ ] Streaming online

[ ] In a public space (i.e. bar or public arena)

[] At home alone

[] At home with others

[ ] Not planning to watch 
5) Can you name any of the individual players on the Canadian National Women's Hockey Team?

( ) Yes, I can name all the players

( ) Yes, I can name some of the players

( ) Yes, I can name one of the players

( ) No I cannot name any of the players

( ) Don't know

6) Watching Canadians play hockey during the Olympics makes me feel (choose one or as many as applicable)

[ ] Proud

[ ] Excited

[ ] Bored

[ ] Embarrassed

[ ] Nothing

[ ] Other - Write In:

7) Do you think Olympic Women's hockey is (choose one or as many as applicable)

[] Exciting

[ ] Violent

[ ] Fast-paced

[] Boring

[ ] Skill-based

[ ] Not as good as the men's game

[ ] As good as the men's game

[ ] Better than the men's game

[ ] Too limited/not enough teams playing

[] I don't watch

[ ] Don't know

[ ] Other - Write In:

8) The National Hockey League chose not to participate in the 2018 Winter Olympics which means their hockey players are not allowed at the Games. Has this, or will this, make you more inclined to watch Women's Olympic Hockey, less inclined to watch Women's Olympic Hockey, or does it make no difference to you?

( ) More inclined to watch Olympic Women's Hockey

( ) Less inclined to watch

( ) Makes no difference to me

( ) Don't know 
9) Are you aware that women also play professional hockey?

() Yes

( ) No

( ) Don't know

10) Which professional women's hockey league have you heard of? (choose one or as many as applicable)

[ ] Canadian Women's Hockey League (CWHL)

[ ] National Women's Hockey League (NWHL)

[ ] I haven't heard of any professional women's hockey leagues

11) Have you ever watched professional women's hockey?

() Yes

() No

() Don't know

12) Why haven't you watched professional women's hockey? (choose one or as many as applicable)

[ ] I didn't know there was professional women's hockey

[ ] I don't watch any hockey

[ ] I don't know where I can watch professional women's hockey

[ ] I don't know when I can watch professional women's hockey

[] I'm not interested

[ ] I don't have enough time

[ ] I have good intentions but forget to watch

[ ] I don't think there are enough teams play/not competitive enough league

[] Women's hockey isn't exciting enough

[ ] I would rather watch NHL Hockey

[ ] Other - Write In:

13) Are you interested in watching professional women's hockey?

() Yes

( ) No

( ) Don't know

14) You would watch professional women's hockey if (choose one or as many as applicable) [ ] there was more advertising/publicity about the games

[ ] I knew where and when to watch games on television

[] I knew how to stream games on the internet/mobile

[ ] I knew more about the players 
[ ] I knew more about the teams

[ ] the games were faster/more exciting

[ ] the games had more hitting/slashing/violence

[] I could watch the games live in an arena

[ ] Other - Write In:

[ ] I wouldn't watch women play professional hockey

15) When you watch women's hockey, does the gender of the players matter to you?

( ) The gender of the players always matters to me

( ) The gender of the players sometimes matters to me

( ) The gender of the players usually does not matter to me

( ) The gender of the players never matters to me

( ) I don't watch

16) Who in your family plays organized hockey? (choose one or as many as applicable)

[] $\mathrm{Me}$

[ ] Partner/Spouse

[ ] Daughter

[] Son

[ ] Mother

[ ] Father

[] Sister

[ ] Brother

[ ] Other - Write In:

[ ] No one in my family plays hockey

17) Do you think women should play professional hockey?

() Yes

( ) No

( ) Don't know

18) Why shouldn't women play professional hockey?

19) Why should women play professional hockey?

20) Do you think that more people should watch professional women's hockey?

() Yes

( ) No 


\section{( ) Don't know}

21) Why should more people watch professional women's hockey?

22) Why shouldn't more people watch professional women's hockey?

23) To which gender identity do you most identify?

[ ] Female

[] Male

[ ] Transgender Female

[ ] Transgender Male

[ ] Gender Variant/Non-Conforming

[ ] Not Listed:

[ ] Prefer not to say

24) What is your age?

( ) 18-24 years old

( ) 25-34 years old

( ) 35-44 years old

( ) 45-54 years old

( ) 55-64 years old

( ) $65-74$ years old

( ) 75 years or older

( ) Prefer not to say

25) What is the highest degree or level of school you have completed? If currently enrolled, highest degree received.

( ) Less than a high school diploma

( ) High school degree or equivalent

( ) Some post-secondary (college/university), no degree

( ) Associate degree (e.g. AA, AS)

( ) Bachelor's degree (e.g. BA, BS)

( ) Master's degree (e.g. MA, MS, Med)

( ) Professional degree (e.g.MD, DDS, DVM)

( ) Doctorate (e.g. PhD, EdD)

( ) Prefer not to say

26) You live in this province/territory 

( ) Alberta
( ) British Columbia
( ) Manitoba
( ) New Brunswick
( ) Newfoundland \& Labrador
( ) Northwest Territories
( ) Nova Scotia
( ) Nunavut
( ) Ontario
( ) Prince Edward Island
( ) Quebec
( ) Saskatchewan
( ) Yukon
( ) I don't live in Canada

27) Which of the following best describes the area you live in?

( ) Urban

( ) Suburban

( ) Rural

28) You may belong to one or more groups on the following list. Are you...? (choose one or as many as applicable)

[] White

[ ] South Asian (e.g., East Indian, Pakistani, Sri Lankan, etc.)

[ ] Chinese

[ ] Black

[ ] First Nation, Inuit, Metis

[ ] Filipino

[ ] Latin American

[] Arab

[ ] Southeast Asian (e.g., Vietnamese, Cambodian, Malaysian, Laotian, etc.)

[ ] West Asian (e.g., Iranian, Afghan, etc.)

[ ] Korean

[] Japanese

[ ] Other - Write In:

[ ] Prefer not to say

29) BY CLICKING SUBMIT SURVEY BELOW, I GIVE MY CONSENT TO PARTICIPATE. IF I PRESS DO NOT SUBMIT, I WILL EXIT THE SURVEY AND NONE OF MY RESPONSES WILL BE SAVED. 


\section{( ) SUBMIT SURVEY \\ ( ) DO NOT SUBMIT}

\section{REMOVE CONSENT}

Thank You! 


\section{Appendix C - Online Survey Consent}

\section{FROZEN OUT: AUDIENCES, AFFECT \& WOMEN'S HOCKEY}

\section{INTRODUCTION AND PURPOSE}

My name is Donna Gall. I am a doctoral student at Ryerson University working with my faculty supervisor, Dr. Jean Bruce, in the graduate program in Communication and Culture. Thank you for taking part in my research study about audiences of women's hockey during and after the Olympics.

\section{WHAT YOU ARE BEING ASKED TO DO}

If you are at least 18 years old and have watched or plan to watch the Canadian National Women's Hockey Team play during the 2018 Winter Olympics, you are being asked to voluntarily complete this on-line survey. It involves questions about watching women's hockey on television or online and should take about 4 minutes to complete. In order for all your answers to be collected you must go to the end of the survey and click 'submit survey'. This will demonstrate your full consent to participation.

\section{POTENTIAL BENEFITS}

There is no direct benefit to you for taking part in this study. It is hoped that the research will build knowledge about audiences for hockey in general and women's hockey in particular. It is hoped that this knowledge may help to grow the audience for women's hockey.

\section{WHAT ARE THE POTENTIAL RISKS TO YOU}

Participating in this online survey poses minimal risk. Losing valuable time is a possible risk so I have kept the questions short and small in number. The questions about viewing habits and women's hockey may cause discomfort over lost personal opportunities to watch or play hockey. You are free to decline to answer any questions you do not wish to answer, or stop participating at any time by closing your browser. If you close your browser before getting to the end of the survey and do not confirm your consent to participate at the end of the survey by clicking the 'submit survey' button your information collected up to that point will not be used.

\section{YOUR IDENTITY WILL BE ANONYMOUS}

This online survey is anonymous and as such will not be collecting information that will easily identify you, like your name or other unique identifiers. Although your Internet Protocol (IP) address can be tracked through the survey platform, the researcher/s will not be collecting this information. Your IP address may be observed only to ensure that one individual is not completing the survey many times and to gather data about general geographic information (city, province) only.

\section{HOW YOUR INFORMATION WILL BE PROTECTED AND STORED}


This survey uses Survey Gizmo which is a United States of America (USA) company. Consequently, USA authorities under the provisions of the Patriot Act may access the survey data. If you would rather participate with an email or paper-based survey please contact the researchers. You will find contact details at the bottom of this page. Please note email or paperbased surveys may allow your identity to be known to the researcher/s but if you select this option your information will be kept confidential.

To further protect your information, data stored by the researcher will be password protected and/or encrypted. Only the researcher/s named in this study will have access to the data as collected. Any future publications will include collective information (i.e., aggregate data). Your individual responses (i.e., raw data) will not be shared with anyone outside of the research team.

When the research is completed, the researcher/s will keep the data for up to 16 months after the study is over.

\title{
INCENTIVES FOR PARTICIPATION
}

You will not be paid for taking part in this study.

\section{YOUR RIGHTS AS A RESEARCH PARTICIPANT}

Participation in research is completely voluntary and you can withdraw your consent at any point up to clicking the submit button at the end of the survey. If you wish to discontinue the survey at any point, simply close your browser and no data will be collected. However, because the survey is anonymous, once you click the submit button at the end of the survey, the researchers will not be able to determine which answers belong to you so your consent cannot be withdrawn after that point.

Please note, that by clicking submit at the end of the study you are providing your consent for participation. By consenting to participate you are not waiving any of your legal rights as a research participant.

\section{QUESTIONS}

If you have any questions about this research, please feel free to contact the researcher.

\author{
RESEARCHER: \\ Donna Gall \\ $\mathrm{PhD}$ Candidate \\ Ryerson University \\ Office \#111 - 111 Gerrard Street \\ Toronto, ON M5B 1G8 \\ dgall@ryerson.ca
}




\section{SUPERVISOR:}

Dr. Jean Bruce

Associate Dean, Graduate Education

Faculty of Communication and Design

Ryerson University

$\underline{416.979 .5000 \# 3590}$

jbruce@ryerson.ca

If you have any questions about your rights or treatment as a research participant in this study, please contact the Ryerson University Research Ethics Board at rebchair@ryerson.ca (416) 9795042.

Please print a copy of this page for your future reference.

By clicking the "Next" button below, I acknowledge I am at least 18 years old and am consenting to participate in this study. 


\section{Appendix D - Online Survey Recruitment Notice}

\section{Ryerson University}

\section{Email, phone or in-person script template}

Hi

My name is Donna Gall. I am a PhD Candidate at Ryerson University in the Communications and Culture. I am contacting you to see if you might be interested in participating in a research study.

This research is being done as part of my $\mathrm{PhD}$ dissertation and my supervisor's name is Dr. Jean Bruce. The focus of the research is learning about the audience for women's hockey during and after the 2018 Olympics.

To participate you need to have watched at least one game of the Canadian National Women's Hockey team during the 2018 Winter Olympics.

If you agree to volunteer you will be asked to participate in an online survey. The online survey is anonymous and will take approximately 10-15 minutes of your time. The link to the survey is www.survey.com.

Your participation is completely voluntary and if you choose not to participate it will not impact your relationship with Hockey Canada, the Canadian Women's Hockey League or Ryerson University.

The research has been reviewed and approved by the Ryerson University Research Ethics Board.

If you are interested in more information about the study or would like to volunteer please reply to this emaildgall@ryerson.ca.

\section{Facebook Post}

Seeking participants for an online audience survey of Women's Hockey during the 2018 Winter Olympics.

This research is being done as part of my $\mathrm{PhD}$ dissertation and my supervisor's name is Dr. Jean Bruce. The focus of the research is learning about the audience for women's hockey during and after the 2018 Olympics. 
To participate you need to have watched at least one game of the Canadian National Women's Hockey team during the 2018 Winter Olympics.

If you agree to volunteer you will be asked to participate in an online survey. The online survey is anonymous and will take approximately 10-15 minutes of your time. The link to the survey is www.survey.com.

Your participation is completely voluntary and if you choose not to participate it will not impact your relationship with Hockey Canada, the Canadian Women's Hockey League or Ryerson University.

The research has been reviewed and approved by the Ryerson University Research Ethics Board.

If you are interested in more information about the study or would like to volunteer please reply to this emaildgall@ryerson.ca.

\section{Twitter Template}

Research Participants Sought \#womenshockeyaudience@RyersonU @FrozenOut 


\section{Appendix E - Focus Group Recruitment Form}

Ryerson

University

\section{COVER LETTER FOR FOCUS GROUP SIGN-UP}

Thank you again so much for signing up to participate in my research study on Women's Hockey as part of my PhD dissertation at Ryerson University in the Communications and Culture department under the supervision of Dr. Jean Bruce. The focus of the research is learning about the audience for women's hockey during and after the 2018 Olympics.

To participate you need to be over the age of 18 and have watched at least one game of the Canadian National Women's Hockey team during the 2018 Winter Olympics.

At the end of the focus group, you will be asked if you are willing to be contacted for a follow up telephone interview. Follow up telephone interviews will be approximately 30 minutes in length.

Your participation is completely voluntary and if you choose not to participate it will not impact your relationship with anyone associated with this study.

Please use the link below to go to an external sign-up page. I have listed the available times and dates for in-person focus groups. Please choose one of the available spots. All focus groups will be held in the Student Learning Centre on Ryerson Campus at 341 Yonge Street in downtown Toronto and will take 1 hour of your time.

After you have chosen your spot, I will send a follow-up email confirming the date, time and place and will include the consent form I will need you to sign when you arrive.

IF YOU CANNOT ATTEND IN PERSON, please indicate your willingness to either participate in an online focus group, a telephone conference call or an individual interview online or by phone. I will then follow-up with you directly by email.

The research has been reviewed and approved by the Ryerson University Research Ethics Board. 


\section{Appendix F -Focus Group Follow Up (1)}

\section{FOCUS GROUP PARTICIPANTS NEEDED FOR DOCTORAL RESEARCH ON WOMEN'S HOCKEY}

ARE YOU:

- A VIEWER OF CANADIAN WOMEN'S HOCKEY DURING THE WINTER OLYMPICS?

- OVER THE AGE OF 18?

If you answered yes to the above noted questions you are invited to volunteer in this audience reception study about women's hockey during and after the 2018 Winter Olympics.

You will be asked to participate in a focus group to talk about watching women's hockey.

Your participation will involve either a 30-45 minute focus group located on Ryerson Campus OR a 30-45 minute online conference call or a 30-45 minute telephone conference call.

At the end of the focus group you will asked if you will be willing to be contacted for a follow-up interview in person or on the telephone. Follow up telephone interviews will be approximately $20-30$ minutes in length.

If you are interested in participating in this study or for more information please contact:

Donna Gall, PhD Candidate at dgall@ryerson.ca.

This study is part of my Doctoral Dissertation.

This research study has been reviewed and approved by the Ryerson University Research Ethics Board.

* Required

1. Email address *

2. NAME

3. EMAIL ADDRESS *

4. CITYIPROVINCE 
5. MOST CONVENIENT DAYS TO MEET/SPEAK

Check all that apply.

$\square$ Monday
$\square$ Tuesday
$\square$ Wednesday
$\square$ Thursday
$\square$ Friday
$\square$ Saturday
$\square$ Sunday

6. MOST CONVENIENT TIME TO MEET/SPEAK

Check all that apply.

$\square$ MORNINGS

AFTERNOONS

1 EVENINGS

Other:

Thank you so much for participating in the research! Don't hesitate to contact me at dgall@ryerson.ca if you require any further information.

Powered by

Google Forms 


\section{Appendix G -Focus Group Follow Up (2)}

\section{Women's Hockey Audience Focus Group Follow-up}

Thank you again so much for signing up to participate in my research study on Women's Hockey. This is part of my PhD dissertation at Ryerson University in the Communications and Culture department under the supervision of Dr. Jean Bruce. The focus of the research is learning about the audience for women's hockey during and after the 2018 Olympics.

To participate you need to be over the age of 18 and have watched at least one game of the Canadian National Women's Hockey team during the 2018 Winter Olympics.

At the end of the focus group, you will be asked if you are willing to be contacted for a follow up telephone interview. Follow up telephone interviews will be approximately 30 minutes in length.

Your participation is completely voluntary and if you choose not to participate it will not impact your relationship with anyone associated with this study.

Below, I have listed the available times and dates for in-person focus groups. Please choose one of the available spots. All focus groups will be held in the Student Learning Centre on Ryerson Campus at 341 Yonge Street in downtown Toronto and will take 1 hour of your time.

After you have chosen your spot, I will send a follow-up email confirming the date, time and place and will include the consent form I will need you to sign when you arrive.

IF YOU CANNOT ATTEND IN PERSON, please indicate your willingness to either participate in an online focus group, a telephone conference call or an individual interview online or by phone. I will then follow-up with you directly by email.

The research has been reviewed and approved by the Ryerson University Research Ethics Board.

Thank you again!

1. Email address *

2. I can attend

Mark only one oval.

Wednesday March 28, 2018 10:30am - 11:30am

Wednesday March 28, 2018 5:30pm - 6:30pm

Thursday March 29, 2018 10:30am - 11:30am

Thursday March 29, 2018 6:00pm - 7:00pm

3. I cannot attend but will

Check all that apply.

Participate in an online focus group

Participate in a telephone conference call

Agree to be interviewed individually by telephone or online

Send me a copy of my responses. 


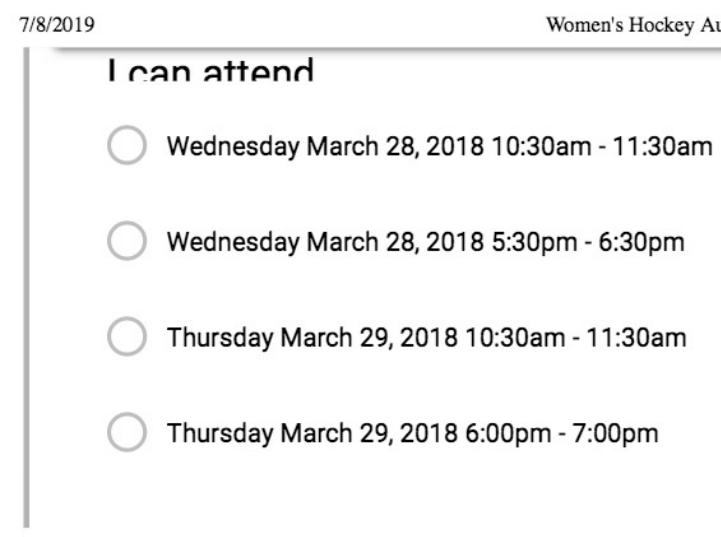

\section{I cannot attend but will}

Participate in an online focus group

Participate in a telephone conference call

Agree to be interviewed individually by telephone or online

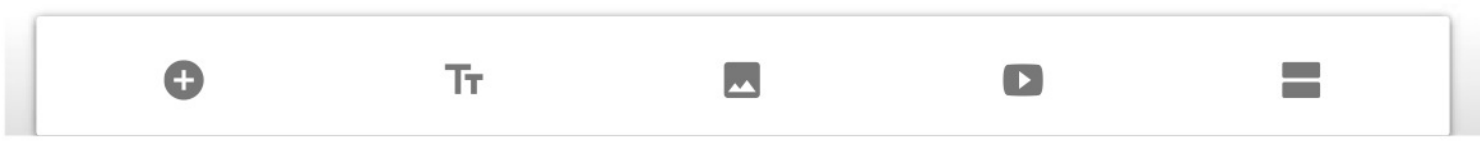




\title{
Appendix H - Focus Group Follow Up (3)
}

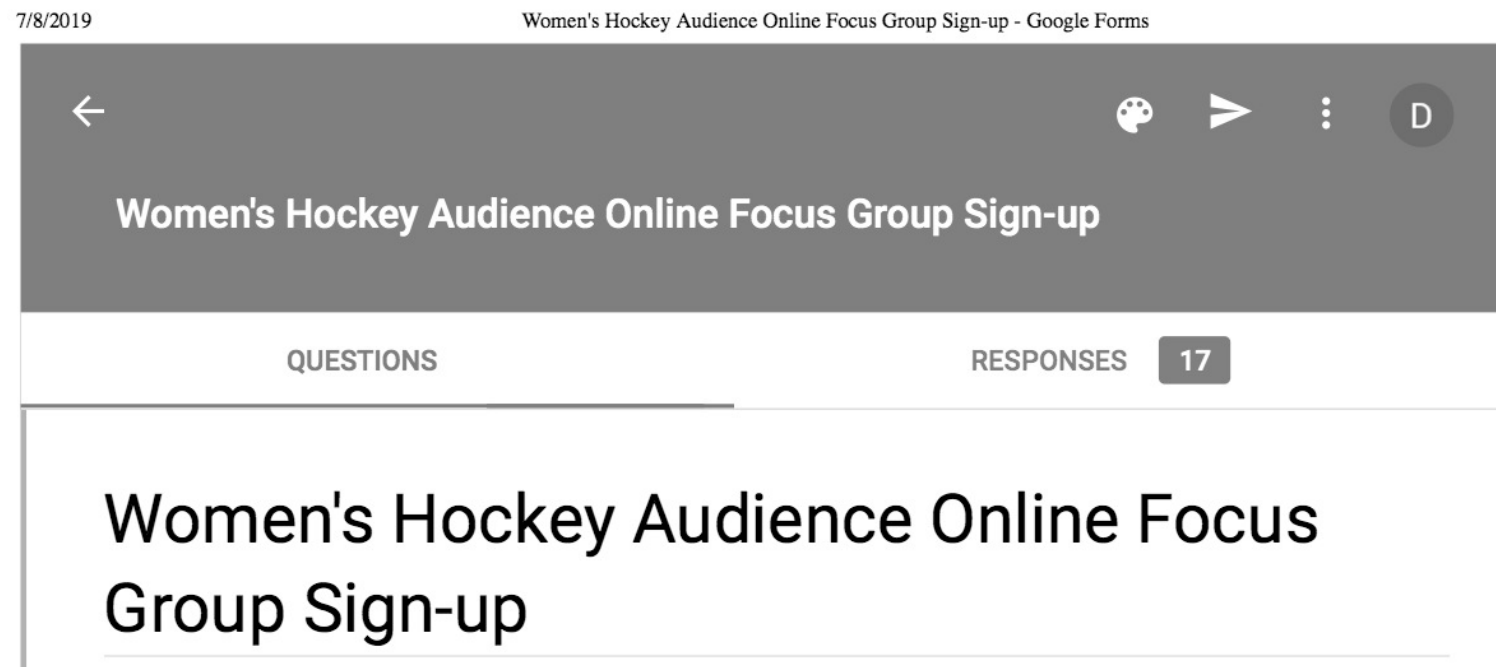

You have indicated that you are willing to participate in an online focus group to discuss Women's Hockey Audiences. Thank you so much for contributing to my doctoral research!

\begin{abstract}
For the online focus groups, I will be using Google Hangout that allows for a small group to meet online. We will communicate with video and/or audio. I have listed below a number of options of particular days and times (EST) over the coming weeks. Please indicate as many time slots that you are able to attend so that I have some options when assembling groups.
\end{abstract}

\begin{abstract}
So that I can try to make the groups as diverse as possible, I am also asking for some demographic information, your age, gender and exposure to hockey. Please note that this information will not be associated with your participation in the focus group. For privacy, I will ensure this information is deleted once I have assigned you to a particular group and you have confirmed that you are able to participate.
\end{abstract}

I will send you an email confirming the day and time as well as a link to the website where the focus group will happen. I will also send you a consent form that I will need you to sign electronically and return to me.

If you are unable or unwilling to participate in a video/audio online conference, I am also setting up a text-based focus group. This will be a website where I will have a series of questions. You can type in your answers as well as comment on other people's answers. This will be a private, closed group by invitation only and will run for a week so that you can participate in your own time and schedule. If this option appeals to you, please indicate below and I will follow up by email with details and the link.

Thank you again so much.

\section{Email address *}

\section{Valid email address}

This form is collecting email addresses. Change settings

I can narticinate in these Video/Audio Online Focus Grouns (nlease choose as<smiles>[C+]</smiles> 


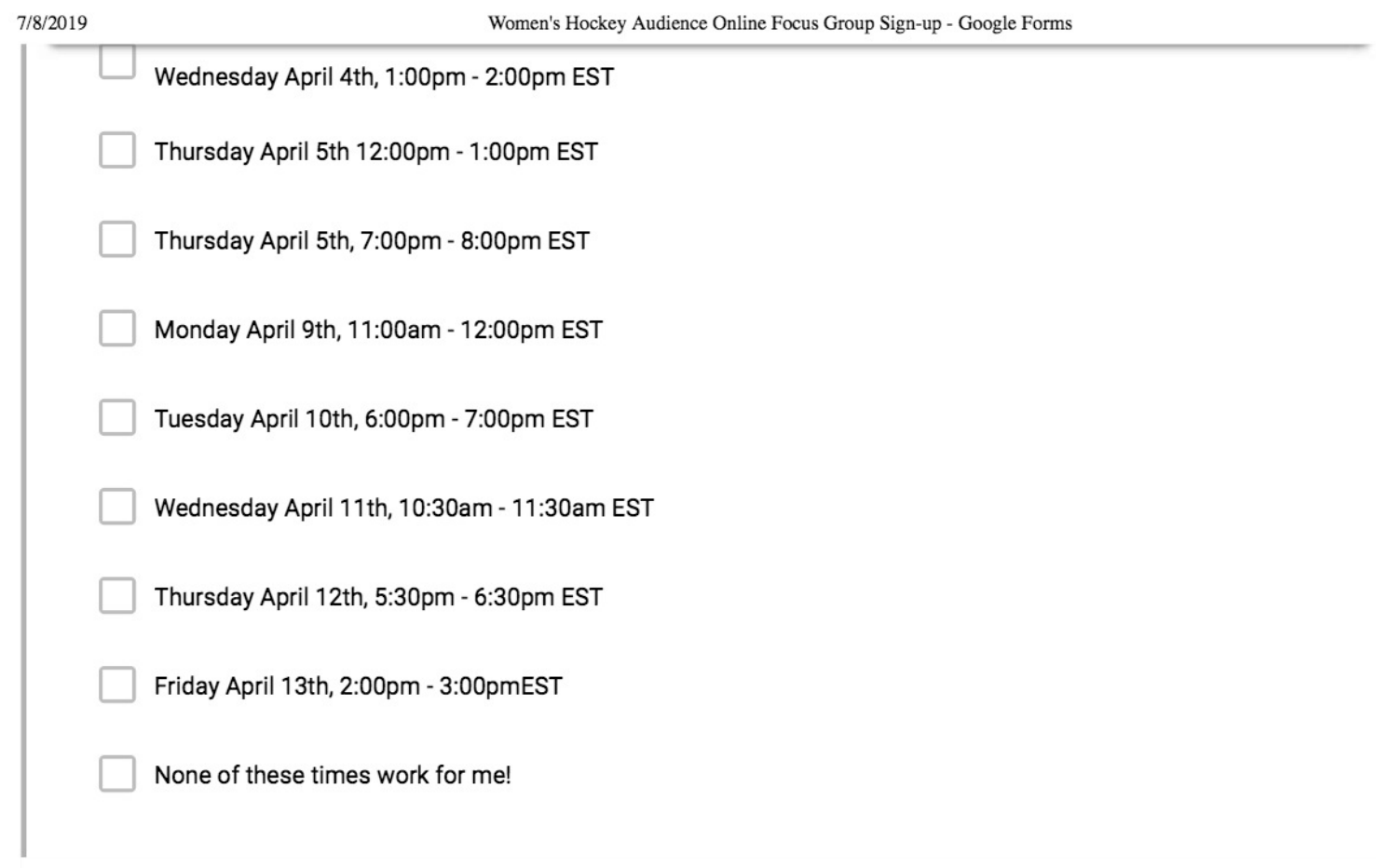

I want to do a text-based online focus group

Yes

\section{To which gender identity do you most identify?}

Female/CIS-Gendered Female

Male/CIS-Gendered Male

Transgender Female

Transgender Male

Gender Variant/Non-Conforming

Prefer not to answer

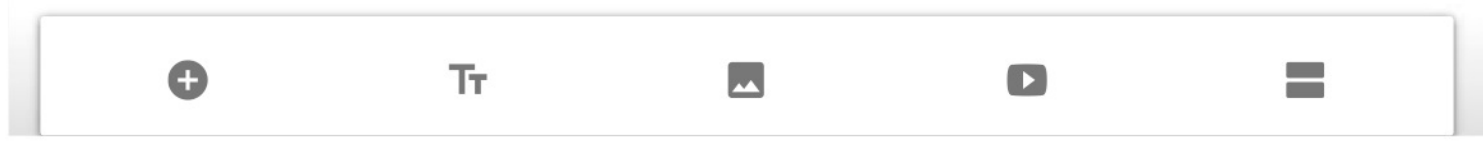




\section{Appendix I - Focus Group Consent}

\section{Ryerson University}

\section{Ryerson University Consent Agreement}

You are being invited to participate in a research study. Please read this consent form so that you understand what your participation will involve. Before you consent to participate, please ask any questions to be sure you understand what your participation will involve.

\section{TITLE OF STUDY: $\quad$ Frozen Out: Audiences, Affect \& Women's Hockey}

INVESTIGATOR: This research study is being conducted by Donna Gall from Communication \& Culture at Ryerson University and supervised by Dr. Jean Bruce, Faculty of Communication and Design, Ryerson University.

If you have any questions or concerns about the research, please feel free to contact Donna Gall at dgall@ryerson.ca or Dr. Bruce at jbruce @ ryerson.ca.

\section{PURPOSE OF THE STUDY}

Millions of Canadians tune in to watch women play hockey during the Olympics but less than $1 \%$ of those viewers continue to watch women play in the professional Canadian Women's Hockey League (CWHL). I want to know more about the audiences for women's hockey beginning during the 2018 Winter Olympics and right through to the broadcast of the Clarkson Cup, the final of the CWHL season. I am recruiting 40 people to participate in 1 of 4 focus groups, 10 people per focus group. I am looking for adult Canadians (18 years or older) who will watch any of the Canadian National Women's hockey game during the 2018 Winter Olympics broadcast on the $\mathrm{CBC}$ or streamed at online site. This research will contribute to the completion of my PhD Dissertation.

\section{WHAT YOU WILL DO}

You will be asked to participate in a focus group to discuss what it is like to watch women's hockey during the 2018 Olympics. The focus group will be one-hour long and will take place in a private meeting room on Ryerson Campus in downtown Toronto. The groups will be scheduled during the day or in the early evening and will occur after the Olympics end sometime between February 25, 2018 and March 31, 2018.

During the focus group I will start the conversation by asking questions about watching women's hockey, I will ask questions about specific games and players, how women's hockey compares to men's hockey, how "Canadian" women's hockey is, if you plan to watch more women's hockey after the Olympics. 
If you indicate that you plan to watch more women's hockey, with your permission, I will contact you to schedule a follow up phone interview to discuss watching women's professional hockey. This telephone interview will take approximately 30 - 40 minutes.

The research findings will be available in my $\mathrm{PhD}$ Dissertation. This is available upon request to all participants.

\section{POTENTIAL BENEFITS}

By participating in this survey you are helping to build knowledge about audiences for hockey in general and women's hockey in particular. It is hoped that this knowledge may help to grow the audience for women's hockey. There is no financial compensation or benefit for participating in this survey. I cannot guarantee, however, that you will receive any benefits from participating in this study.

\section{POTENTIAL RISKS}

Participating in this focus group poses minimal risk. The questions about viewing habits and women's hockey may cause discomfort over lost personal opportunities to watch or play hockey. Conversations about gender and sport may cause discomfort with differing opinions about women's rights or abilities to play.

\section{CONFIDENTIALITY}

I will ask for your personal information including name, contact information, age and gender. However, I will keep your participation in the focus group confidential. I will assign you a numeric code so that any statements you make during the focus group will not be identifiable. This coding information will be kept secure in a locked office. I will take notes during the focus group and this will also be kept secure in a locked office. I will keep these notes until the completion of my PhD Dissertation after which they will be shredded.

With your permission I will video and/or audio record the focus group for note taking purposes only. Only my supervisor Dr. Jean Bruce and I will have access to this video or audio material. Once I have transcribed the conversation, I will digitally wipe the video/audio tapes. The transcriptions will be stored on an external hard drive kept in a locked office. The data on the hard-drive will be encrypted and password protected. The data will be kept until the completion of my PhD Dissertation after which it will be digitally wiped. If you would like to review or edit your focus group statements I will provide those transcripts for you to review up you one month after the focus group.

\section{INCENTIVES FOR PARTICIPATION}

There is no financial or in-kind incentive for participating in the survey other than the potential benefits as detailed above.

Upon request I will also provide you with a final copy of my completed dissertation.

\section{COSTS TO PARTICIPATION}

The focus group will be held on Ryerson Campus in downtown Toronto. I will offer reimbursement for one hour parking up to $\$ 10$ or two TTC tokens to reimburse transportation charges. 


\section{VOLUNTARY PARTICIPATION AND WITHDRAWAL}

Participation in this study is completely voluntary. You can choose whether to be in this study or not. If any question makes you uncomfortable, you can refuse to answer that question. You may stop participating at any time. If you choose to stop participating, you may also choose to not have your data included in the study. Your choice of whether or not to participate will not influence your future relations with Ryerson University or the investigator Donna Gall or supervisor Jean Bruce involved in the research.

OUESTIONS ABOUT THE STUDY: If you have any questions about the research now, please ask. If you have questions later about the research, you may contact:

INVESTIGATOR: Donna Gall

PhD Candidate

Ryerson University

Office \#111 - 111 Gerrard Street

Toronto, ON M5B 1G8

dgall@,ryerson.ca

SUPERVISOR: $\quad$ Dr. Jean Bruce

Associate Dean, Graduate Education

Faculty of Communication and Design

Ryerson University

$\underline{416.979 .5000 \# 3590}$

jbruce@ryerson.ca

This study has been reviewed by the Ryerson University Research Ethics Board. If you have questions regarding your rights as a participant in this study please contact:

Research Ethics Board

c/o Office of the Vice President, Research and Innovation

Ryerson University

350 Victoria Street

Toronto, ON M5B 2K3

416-979-5042

rebchair@ryerson.ca 
FROZEN OUT: AUDIENCE, AFFECT \& WOMEN'S HOCKEY

\section{CONFIRMATION OF AGREEMENT:}

Your signature below indicates that you have read the information in this agreement and have had a chance to ask any questions you have about the study. Your signature also indicates that you agree to participate in the study and have been told that you can change your mind and withdraw your consent to participate at any time. You have been given a copy of this agreement.

You have been told that by signing this consent agreement you are not giving up any of your legal rights.

Name of Participant (please print)

Signature of Participant Date

I agree to be audio and/or video recorded for the purposes of this study. I understand how these recordings will be stored and destroyed.

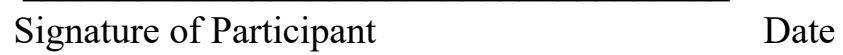

I agree to allow the researcher to contact me to schedule a follow-up telephone interview. I can however change my mind and withdraw this consent at any time.

Date 


\section{Bibliography}

Abdel-Shehid, G. (2005). Who da' man? Black masculinities and sporting cultures. Canadian Scholars' Press.

Abrams, K. M. \& Gaiser, T.J. (2017). Online Focus Groups. In Nigel G. Fielding, Raymond M. Lee, \& Grant Blank (Eds.) The Sage Handbook of Online Research Methods (pp. 435449).

Adams, C. (2008). "Queens of the Ice Lanes": The Preston Rivulettes and Women's Hockey in Canada, 1931-1940. Sport History Review, 39(1), 1-29.

Adams, C., \& Leavitt, S. (2018). 'It's just girls' hockey': Troubling progress narratives in girls' and women's sport. International review for the sociology of sport, 53(2), 152-172.

Adams, C., \& Stevens, J. (2007). Change and grassroots movement: Reconceptualising women's hockey governance in Canada. International Journal of Sport Management and Marketing, 2(4), 344-361. doi:10.1504/IJSMM.2007.013710

Adams, M. L. (2006). The Game of Whose Lives? Gender, Race, and Entitlement in Canada's 'National Game'. In R.S. Gruneau \& D. Whitson (Eds.), Artificial ice: Hockey, culture, and commerce (pp. 71-84). University of Toronto Press.

Adams, Mary Louise. (2011). Artistic impressions: Figure skating, masculinity, and the limits of sport. University of Toronto Press.

Adriaanse, J. A., \& Claringbould, I. (2016). Gender equality in sport leadership: From the Brighton Declaration to the Sydney Scoreboard. International Review for the Sociology of Sport, 51(5), 547-566.

Adriaanse, J., \& Schofield, T. (2014). The impact of gender quotas on gender equality in sport governance. Journal of Sport Management, 28(5), 485-497.

Afoko, C. (2018, September 10). Serena Williams's treatment shows how hard it is to be a black woman at work. The Guardian. Retrieved from https://www.theguardian.com/commentisfree/2018/sep/10/serena-williams-black-womanwork-tennis-discrimination

Ahmed, S. (2004). The Cultural Politics of Emotion Routledge.

All-Star Game. (2014). Retrieved from http://www.thecwhl.com/allstar/history

Allain, K. A. (2008). "Real fast and tough": The construction of Canadian hockey masculinity. Sociology of Sport Journal, 25(4), 462-481.

Allain, K. A. (2011). Kid Crosby or Golden Boy: Sidney Crosby, Canadian national identity, and the policing of hockey masculinity. International Review for the Sociology of Sport, $46(1), 3-22$. 
Allain, K. A. (2014). 'What happens in the room stays in the room': Conducting research with young men in the Canadian Hockey League. Qualitative Research in Sport, Exercise and Health, 6(2), 205-219.

Allen-Collinson, J. (2011). Feminist phenomenology and the woman in the running body. Sport, ethics and philosophy, 5(3), 297-313.

Allen, K. \& Frisby, C. (2017). A Content Analysis of Micro Aggressions in News Stories about Female Athletes Participating in the 2012 and 2016 Summer Olympics. Journal of Mass Communication \& Journalism. DOI: 10.4172/2165-7912.1000334

Alvarez, A. (2018, April 14). Should female athletes sue the networks for equal coverage? The Guardian. Retrieved from https://www.theguardian.com/sport/2018/apr/14/womensathletes-lawsuit-equal-coverage-title-ix

Anderson, C. A. (2016). Media violence effects on children, adolescents and young adults. Health Progress (Saint Louis, Mo.), 97(4), 59-62.

Anderson, E. (2002). Openly Gay Athletes: Contesting Hegemonic Masculinity in a Homophobic Environment. Gender \& Society 16(6): 860—77.

Andrews, D. L. (1996). The fact(s) of Michael Jordan's blackness: Excavating a floating racial signifier. Sociology of Sport Journal, 13(2), 125-158.

Ang, I. (1985). Watching Dallas: Soap opera and the melodramatic imagination. Routledge.

Ang, I. (1990). Culture and communication: Towards an ethnographic critique of media consumption in the transnational media system. European Journal of Communication, 5(2), 239-260.

Ang, I. (1991). Desperately seeking the audience Routledge.

Ang, I. (1995) The nature of the audience. In J. Downing, A. Mohammadi, \& A. SrebernyMohammadi (Eds.), Questioning the Media: A Critical Introduction (2nd ed.) ( pp. 207220). Sage.

Ang, I. (2007). Watching Dallas: Soap opera and the melodramatic imagination (2nd ed.). Routledge.

Annie, B. (2018, July 25). Women's Hockey Wednesday: Brenda Andress leaves behind a strong CWHL. Vox Media. Retrieved from https://www.pensionplanpuppets.com/2018/7/25/17600678/womens-ice-hockeywednesday-brenda-andress-leaves-cwhl-commissioner-jayna-hefford-one-league-inferno

Antunovic, D. (2019). "we wouldn't say it to their faces": Online harassment, women sports journalists, and feminism. Feminist Media Studies, 19(3), 428-442.

doi:10.1080/14680777.2018.1446454 
Antunovic, D., \& Hardin, M. (2012). Activism in Women's sports blogs: Fandom and feminist potential. International Journal of Sport Communication, 5(3), 305-322.

doi:10.1123/ijsc.5.3.305

Antunovic, D., \& Hardin, M. (2015). Women and the blogosphere: Exploring feminist approaches to sport. International Review for the Sociology of Sport, 50(6), 661-677. doi:10.1177/1012690213493106

Armour, N. (2018, September 09). Instead of triumphing, Serena Williams diminished herself with behaviour at the U.S. Open. USA Today. Retrieved from https://www.usatoday.com/story/sports/columnist/nancy-armour/2018/09/08/serenawilliams-didnt-behave-like-champion-us-open-loss/1243879002/

Armstrong, J. (2017, June 30). Tokyo 2020 will attract youth, offer gender equality: IOC VP. CTV News. Retrieved from https:/www.ctvnews.ca/sports/tokyo-2020-will-attract-youthoffer-gender-equality-ioc-vp-1.3483377

Arthur, B. (2018, February 19). It's Canada and the United States for women's hockey gold. The Toronto Star. Retrieved from https://www.thestar.com/sports/olympics/2018/02/19/canadian-women-advance-to-goldmedal-hockey-game-vs-archrival-us.html

Arthur, B. (2018, February 20). 'Beat them on the ice': The rise of Brigette Lacquette, the first Indigenous woman on Canada's Olympic hockey team. The Toronto Star. Retrieved from https://www.thestar.com/sports/olympics/2018/02/20/beat-them-on-the-ice-the-rise-ofbrigette-lacquette-the-first-indigenous-woman-on-canadas-olympic-hockey-team.html

Atkinson, M. (2010). It's still part of the game: Violence and masculinity in Canadian ice hockey. In L. K. Fuller (Ed.), Sexual Sports Rhetoric: Historical and Media (pp. 15-30). Peter Lang Inc., International Academic Publishers.

Ayala, E. (2017, May 22). Four American Women of Color Navigating Hockey's Whiteness. The Victory Press. Retrieved from https:/victorypress.org/2017/05/22/four-american-womenof-color-navigating-hockeys-whiteness

Bacon-Smith, C. (1992). Enterprising women: Television fandom and the creation of popular myth University of Pennsylvania Press.

Bailey, R., Wellard, I., \& Dismore, H. (2005). Girls' Participation in Physical Activities and Sports: Benefits, Patterns, Influences, and Ways Forward. World Health Organization Centre for Physical Education and Sport. Retrieved 18 August 2016 from http://www.icsspe.org/sites/default/files/Girls.pdf.

Balsamo, A. (1995). Forms of technological embodiment: Reading the body in contemporary culture. Body \& Society, 1(3-4), 215-237.

Barber, J. (2013). Montreal Canadiens: Thrilling Stories from Canada's Famous Hockey Franchise. James Lorimer \& Company. 
Barker, M. (2019). How shall we measure our progress? on paradigms, metaphors, and meetings in audience research. Television \& New Media, 20(2), 130-141.

Barthes, R. (1972). Mythologies 1957. Trans. Annette Lavers. New York: Hill and Wang, 30206.

Bartos, R. (1989). Marketing to Women Around the World. Harvard Business School Press.

Bateson, G. (1972). The logical categories of learning and communication. Steps to an Ecology of Mind, 279-308.

Bausinger, H. (1984). Media, technology and daily life. Media Culture \& Society, 6(4), 343-351.

Belam, M. (2018, February 23). Canada's Jocelyne Larocque apologises for removing silver medal on podium. Retrieved from https://www.theguardian.com/sport/2018/feb/23/canada-jocelyne-larocque-apologisesfor-removing-silver-medal-on-podium

Bélanger, A., \& Valois-Nadeau, F. (2015). From the Shinny Pond to the Bell Centre: Reweaving the Myth of the Sainte-Flanelle. In C. Boissinot, N. Baillargeon, \& S. Irving, (Eds.) Hockey and philosophy (pp. 71-96). University of Ottawa Press.

Bellegarde, B. (2018, January 23). Meet Brigette Lacquette, the 1st First Nations woman on Canada's Olympic hockey team. CBC News. Retrieved from https://www.cbc.ca/news/indigenous/brigette-lacquette-cote-first-nation-olympic-hockeyteam-1.4499136

Bem, S. L. (1981). Gender schema theory: A cognitive account of sex typing. Psychological Review, 88(4), 354-364. doi:10.1037/0033-295X.88.4.354

Bennett, D. (2018, February 6). Hockey is for Everyone: The Jessica Platt Story. Sportsnet. Retrieved April 15, 2019, from https://www.sportsnet.ca/hockey/nhl/hockey-everyonejessica-platt-story/

Berelson, B. R., Lazarsfeld, P. F., McPhee, W. N., \& McPhee, W. N. (1954). Voting: A Study Of Opinion Formation In A Presidential Campaign. University of Chicago Press.

Berkman, S. (2017, September 01). Women's Hockey League Will Pay Players for First Time. New York Times. Retrieved from https://www.nytimes.com/2017/09/01/sports/hockey/womens-hockey-league-will-payplayers-for-first-time.html

Bernstein, A. (2002). Is it time for a victory lap?: Changes in the media coverage of women in sport. International Review for the Sociology of Sport, 37(4), 415-428. doi:10.1177/1012690202037004022

Bertrand, J. T., Brown, J. E., \& Ward, V. M. (1992). Techniques for analyzing focus group data. Evaluation Review, 16(2), 198-209. doi:10.1177/0193841X9201600206

Beville, H. M. (1988). Audience ratings: Radio, television, and cable. Psychology Press. 
Billings, A. C. (2008). Olympic media: Inside the biggest show on television. Routledge.

Billings, A. C., \& Eastman, S. T. (2002). Selective representation of gender, ethnicity, and nationality in American television coverage of the 2000 Summer Olympics. International review for the sociology of sport, 37(3-4), 351-370.

Billings, A. C., \& Hardin, M. (2014). Defining ubiquity: Introduction to the Routledge Handbook of Sport and New Media. In Routledge Handbook of Sport and New Media (pp. 19-22). Routledge.

Billings, A. C., \& Hundley, H. L. (2010). Examining identity in sports media (pp. 1-15). Los Angeles, CA: Sage Publications.

Billings, A. C., \& Young, B. D. (2015). Comparing flagship news programs: Women's sport coverage in ESPN's SportsCenter and FOX Sports Live. Electronic News, 9(1), 3-16.

Billings, A. C., Angelini, J. R., \& Wu, D. (2011). Nationalistic notions of the superpowers: Comparative analyses of the American and Chinese telecasts in the 2008 Beijing Olympiad. Journal of Broadcasting \& Electronic Media, 55(2), 251-266.

Billings, A. C., Brown, K. A., \& Brown, N. A. (2013). 5,535 hours of impact: Effects of Olympic media on nationalism attitudes. Journal of Broadcasting \& Electronic Media, 57(4), 579-595.

Bird, S. E. (2003). Audience in everyday life: Living in a media world Routledge.

Bird, S. E. (2012). Everyday Life: Beyond Audience Response. The Routledge companion to news and journalism (Revised ed.), (pp. 417-427). Routledge.

Birrell, S. (2000). Feminist theories for sport. In J. Coakley \& E. Dunning (Eds.), Handbook of sports studies (pp. 61-76). Sage.

Birrell, S., \& Cole, C. L. (1994). Women, Sport, and Culture. Champaign, Il: Human Kinetics.

Birrell, S., \& Loy Jr., J. W. (1979). Media Sport: Hot and Cool. International Review of Sport Sociology, 14(1), 5-19.

Blain, N., \& Bernstein, A. (2003). Sport, Media, Society: Global and Local Dimensions. Frank Cass.

Blake, J. (2010). Canadian Hockey Literature: A Thematic Study. University of Toronto Press.

Blinde, E. M., \& Taub, D. E. (1992). Women athletes as falsely accused deviants: Managing the lesbian stigma. Sociological Quarterly, 33(4), 521-533.

Blumenthal, D. (1997). Women And Soap Opera: A Cultural Feminist Perspective Praeger.

Bociurkiw, M. (2011). Feeling Canadian: Television, Nationalism, And Affect. Wilfrid Laurier Univ. Press. 
Bogart, L. (1996). Research as an instrument of power. American Communication Research: The Remembered History, 135-146.

Bourdieu, P. (1978). Sport and social class. Information (International Social Science Council), 17(6), 819-840.

Boyle, R., \& Haynes, R. (2009). Power play: Sport, the media and popular culture (2nd ed.) Edinburgh University Press.

Brady, R. (2017, November 09). Jayna Hefford looks to future of women's hockey as she takes place in Hall of Fame. The Globe and Mail. Retrieved from https://www.theglobeandmail.com/sports/article-canadian-national-team-pillar-heffordlooks-to-future-of-womens/

Brady, R. (2018a, March 07). Jessica Platt's journey: How hockey helped a transgender player find herself. The Globe and Mail. Retrieved from https://www.theglobeandmail.com/sports/hockey/transgender-hockey-player-jessicaplatts-journey-toward-a-newidentity/article38181760/

Brady, R. (2018b, May 25). Canadian Women's Hockey League will begin paying its players. The Globe and Mail. Retrieved from https://www.theglobeandmail.com/sports/hockey/cwhl-will-pay-its-players-for-the-firsttime-starting-this-season/article36139819/

Branch, J. (2015, July 27). Dutee Chand, Female Sprinter With High Testosterone Level, Wins Right to Compete. The New York Times. Retrieved from https://www.nytimes.com/2015/07/28/sports/international/dutee-chand-female-sprinterwith-high-male-hormone-level-wins-right-to-compete.html

Brennan, T. (2004). The Transmission Of Affect. Cornell University Press.

Brooks, A. (2002). Postfeminisms: Feminism, Cultural Theory And Cultural Forms. Routledge.

Brown, M. E. (1994). Soap Opera And Women's Talk: The Pleasure Of Resistance Sage Publications.

Bruce, J. (2009). Home improvement television: Holmes on homes makes it right. Canadian Journal of Communication, 34(1), 79. doi:10.22230/cjc.2009v34n1a2181

Bruce, T. (1998). Audience frustration and pleasure: Women viewers confront televised women's basketball. Journal of Sport and Social Issues, 22(4), 373-397.

Bruce, T. (2004). Marking the boundaries of the 'normal' in televised sports: The play-by-play of race. Media, Culture \& Society, 26(6), 861-879.

Bruce, T. (2016). New rules for new times: Sportswomen and media representation in the third wave. Sex Roles, 74(7-8), 361-376. 
Bruce, T., \& Scott-Chapman, S. (2010). New Zealand: Intersections of nationalism and gender in Olympics newspaper coverage. In Bruce, T., Hovden, J., \& Markula, P. (Eds.) Sportswomen at the Olympics (pp. 273-287). Sense Publishers.

Brunsdon, C. (1981). 'Crossroads' Notes on Soap Opera. Screen, 22(4), 32-37.

Brunsdon, C. (2000). The feminist, the housewife, and the soap opera. Oxford University Press.

Brunsdon, C., \& Morley, D. G. (1978). Everyday Television: Nationwide. British Film Institute.

Bryant, J., \& Raney, A. A. (2000). Sports on the screen. In D. Zillmann \& P. Vorderer (Eds.), LEA's communication series. Media entertainment: The psychology of its appeal (pp. 153-174). Lawrence Erlbaum Associates Publishers.

Bryant, J., \& Zillmann, D. (2009). A retrospective and prospective look at media effects. In R.L. Nabi \& M.B. Oliver, (Eds.) The Sage Handbook Of Media Processes And Effects (pp. 917). Sage.

Buckingham, D. (2008). Children and media: A cultural studies approach. The international handbook of children, media and culture, 219-236. Burnett, C. (2001). Whose game is it anyway? Power, play and sport. Agenda, 16(49), 71-78.

Burrow, S. (2016). Trampled autonomy: Women, athleticism, and health. IJFAB: International Journal of Feminist Approaches to Bioethics, 9(2), 67-91.

Burton, L. J. (2015). Underrepresentation of women in sport leadership: A review of research. Sport Management Review, 18(2), 155-165.

Butler, J. (1993). Critically queer. GLQ: A Journal of Lesbian and Gay Studies, 1(1), 17-32. doi:10.1215/10642684-1-1-17

Butler, J. (1999). Gender Trouble: Feminism And The Subversion Of Identity (10th ed.). Routledge.

Byrne, B. (2004). Qualitative interviewing. Researching Society and Culture, 2, 179-192.

CAAWS (Canadian Association for the Advancement of Women and Sport and Physical Activity) (2016). "Women in Sport: Fuelling a Lifetime of Participation. A Report on the Status of Female Sport Participation in Canada." https://www.caaws.ca/e/wpcontent/uploads/2016/03/FWC_ResearchPublication_EN_7March2016.pdf

Cahn, S. K. (1994). Coming On Strong: Gender And Sexuality In Twentieth-century Women's Sport Free Press.

Cairnie, J. (2019). Truth and reconciliation in postcolonial hockey masculinities. Canadian Literature, (237), 103-183.

Calderón, C. A., Álvarez, M., \& Mariño, M. V. (2019). Distributed Supervised Sentiment Analysis of Tweets: Integrating Machine Learning and Streaming Analytics for Big Data Challenges in Communication and Audience Research. Empiria: Revista de metodología de ciencias sociales, (42), 113-136. 
Callegaro, M. (2008). Social desirability. Encyclopaedia Of Survey Research Methods, 826-827.

Campbell, K. K. (1989). The sound of women's voices. Quarterly Journal of Speech, 75(2), 212220.

Campbell, M. (2019). Caster Semenya goes to court for the right to run. The Toronto Star. Retrieved from https://www.thestar.com/sports/sports-prism/2019/02/27/caster-semenyagoes-to-court-for-the-right-to-run.html

Canada. (1991). Broadcasting Act (1991, c. 11). Retrieved from: http://lawslois.justice.gc.ca/eng/acts/B-9.01/FullText.html

Canada's Women's Olympic Team to Pay Homage to Rose Cherry. (1997, November 7). Hockey Canada. Retrieved from http://www.hockeycanada.ca/en-ca/news/1997-nr-113-en

Canadian Broadcasting Corporation. (2019, September 9). The Complete Lineup Of Pairs Prepares To Hit The Ice On Cbc's Battle Of The Blades, Premiering September 19. [Press Release]. Retrieved from: https://www.cbc.ca/mediacentre/press-release/thecomplete-lineup-of-pairs-prepares-to-hit-the-ice-on-cbcs-battle-of-the

Canadian Heritage. (2019). The Government of Canada Is Taking Concrete Steps to Achieve Gender Equity in All Levels of Sport [Press Release]. Retrieved from https://www.canada.ca/en/canadian-heritage/news/2019/03/the-government-of-canada-istaking-concrete-steps-to-achieve-gender-equity-in-all-levels-of-sport.html

Canadians less interested in Olympic hockey without NHL players: Angus Reid. (2018, February 7). Corus Entertainment. Retrieved from https://1045freshradio.ca/news/4010926/canadians-less-interested-in-olympic-hockeywithout-nhl-players-angus-reid/

Caprino, K. (2018, September 10). Serena Williams, Sexism And The Immense Power Of Speaking Up. Retrieved from https://www.forbes.com/sites/kathycaprino/2018/09/10/serena-williams-sexism-and-theimmense-power-of-speaking-up/\#2a8fcaf655d5

Castelnuovo, S., Guthrie, S. R., Eileen, Laura McDonagh, \& Pappano. (2008). Why separate is not equal in sports: Playing with the boys. Politics \& Gender, 4(4), 655.

Caudwell, J. (2007). Queering the field? the complexities of sexuality within a lesbian-identified football team in England. Gender, Place \& Culture, 14(2), 183-196.

doi:10.1080/09663690701213750

Caudwell, J. (2014). [Transgender] young men: Gendered subjectivities and the physically active body. Sport, Education and Society, 19(4), 398-414.

Cavanagh, S. L., \& Sykes, H. (2006). Transsexual bodies at the Olympics: The international Olympic committee's policy on transsexual athletes at the 2004 Athens summer games. Body \& Society, 12(3), 75-102. doi:10.1177/1357034X06067157 
CBC touts Canadian ratings for Winter Games. (2018, February 27). Windsor News. Retrieved from https://www.ourwindsor.ca/community-story/8288756-cbc-touts-canadian-ratingsfor-winter-games/

CBC Unveils Star-studded Roster Of Commentators, Analysts And Reporters For Englishlanguage Coverage Of Pyeongchang 2018 - CBC Media Centre. (n.d.). Retrieved from https://www.cbc.ca/mediacentre/press-release/cbc-unveils-star-studded-roster-ofcommentators-analysts-and-reporters-for

Chalabaev, A., Sarrazin, P., Fontayne, P., Boiché, J., \& Clément-Guillotin, C. (2013). The influence of sex stereotypes and gender roles on participation and performance in sport and exercise: Review and future directions. Psychology of Sport and Exercise, 14(2), 136-144.

Chaney, D. (1972). Processes of Mass Communication. London: Macmillan.

Charmaz, K. \& Henwood, K. (2008). Grounded Theory. In C. Willig \& W. Stainton Rogers (Eds.) The SAGE handbook of qualitative research in psychology (pp. 240-260). Sage.

Chidley-Hill, J. (2018, January 06). Sweden's captain throws silver medal into crowd after frustrating loss to Canada. Rogers Media. Retrieved from https://www.680news.com/2018/01/06/swedens-captain-throws-silver-medal-into-crowdafter-frustrating-loss-to-canada/

Chikarovski, K. (2015). If you broadcast it, they will watch. Daily Telegraph. Retrieved from http://www.dailytelegraph.com.au/rendezview/womens-sport-if-you-broadcast-it-theywill-watch/news-story/3b10bf4d23ec201766284df60b741c24

Clark, K., Ferkins, L., Smythe, L., \& Jogulu, U. (2018). Valuing the lived experience: a phenomenological study of skiing. Sport in Society, 21(2), 283-301.

Clarkson Cup in limbo over ownership rights. (2007, October 31). The Toronto Star. Retrieved from https://www.thestar.com/sports/2007/10/31/clarkson_cup_in_limbo_over_ownership_rig hts.html

Clipperton, J. (2019, February 12). 'It hurts to think about': Canada's men's hockey team reflects on Olympic bronze, CBC Sports. Retrieved from https://www.cbc.ca/sports/hockey/pyeongchang-lookback-mens-hockey-1.5015266

Clipperton, J. (2019, January 20). Merger, equality on players' minds at CWHL all-star game, CBC Sports. Retrieved from https:/www.cbc.ca/sports/hockey/cwhl-all-stargame-toronto-1.4986030

Clough, P. T. (2008). The affective turn: Political economy, biomedia and bodies. Theory, Culture \& Society, 25(1), 1-22.

Clough, P., \& Nutbrown, C. (2012). A student's guide to methodology. Sage.

Coakley J.J. (2004) Sports in Society: Issues and Controversies. McGraw-Hill. 
Cohan, N. (2019). We Average Unbeautiful Watchers: Fan Narratives And The Reading Of American Sports. University of Nebraska Press.

Cohen, J. H., \& Semerjian, T. Z. (2008). The collision of trans-experience and the politics of women's ice hockey. International Journal of Transgenderism, 10(3-4), 133-145.

Cole, C. L. (1993). Resisting the canon: Feminist cultural studies, sport, and technologies of the body. Journal Of Sport And Social Issues, 17(2), 77-97.

Cole, C. L. (2000). One chromosome too many? In K. Schaffer \& S. Smith (Eds.)The Olympics At The Millennium: Power, Politics And The Games. New Brunswick, NJ: Rutgers University Press. pp. 128-146.

Cole, C. L., \& Denny III, H. (1994). Visualizing deviance in post-Reagan America: Magic Johnson, AIDS, and the promiscuous world of professional sport. Critical Sociology, 20(3), 123-147.

Cole, C. L., \& Hribar, A. (1995). Celebrity feminism: Nike style post-Fordism, transcendence, and consumer power. Sociology of Sport Journal, 12(4), 347-369.

Coles, G. (2000). Docusoap: actuality and the serial format. In M. Llewellyn-Jones And B. Carson Frames And Fictions on Television. The Politics of Identity within Drama (pp. 27-39). University of Chicago Press.

Comisky, P., Bryant, J., \& Zillmann, D. (1977). Commentary as a substitute for action. Journal of Communication, 27(3), 150-153.

Connell, R. (2008). Masculinity construction and sports in boys' education: A framework for thinking about the issue. Sport, Education and Society, 13(2), 131-145.

Connell, R. W., \& Messerschmidt, J. W. (2005). Hegemonic masculinity: Rethinking the concept. Gender and Society, 19(6), 829-859. doi:10.1177/0891243205278639

Cooky, C., \& Dworkin, S. L. (2013). Policing the boundaries of sex: A critical examination of gender verification and the caster Semenya controversy. The Journal of Sex Research, 50(2), 103-111. doi:10.1080/00224499.2012.725488

Cooky, C., Messner, M. A., \& Hextrum, R. H. (2013). Women play sport, but not on TV: A longitudinal study of televised news media. Communication \& Sport, 1(3), 203-230.

Cooky, C., Messner, M. A., \& Musto, M. (2015). "It's dude time!” A quarter century of excluding women's sports in televised news and highlight shows. Communication \& Sport, 3(3), 261-287.

Corcoran, T. (2019, May 08). Want to save women's pro hockey? 'Equity tax' the NHL's millionaires. The Financial Post. Retrieved from https://business.financialpost.com/opinion/terence-corcoran-want-to-save-womens-prohockey-equity-tax-the-nhls-millionaires 
Costa, D. M., \& Guthrie, S. R. (1994). Women and Sport: Interdisciplinary Perspectives. Human Kinetics.

Couldry, N., \& Hepp, A. (2018). The Mediated Construction Of Reality. John Wiley \& Sons.

Cox, N. B. (2012). Femme dysfunction is pure gold" A feminist political economic analysis of Bravo's "The Real Housewives [Doctoral Dissertation]. The Florida State University.

Crane, J., \& Temple, V. (2015). A systematic review of dropout from organized sport among children and youth. European Physical Education Review, 21(1), 114-131.

Crawford, G., \& Gosling, V. K. (2004). The Myth of the 'Puck Bunny' Female Fans and Men's Ice Hockey. Sociology, 38(3), 477-493.

Creamer, E. G. (2018). Enlarging the conceptualization of mixed method approaches to grounded theory with intervention research. American Behavioural Scientist, 62(7), 919934.

Creedon, P. J. (Ed.). (1994). Women, Media And Sport. Sage.

Crenshaw, K. (1991). Mapping the margins: Intersectionality, identity politics, and violence against women of color. Stanford Law Review, 43(6), 1241-1299. doi:10.2307/1229039

Creswell, J. W., \& Maietta, R. C. (2002). Qualitative research. In D. C. Miller \& N. J. Salkind (Eds.), Handbook Of Social Research (pp. 143-184). Thousand Oaks, CA: Sage.

Creswell, J. W., \& Plano Clark, V. L. (2011). Designing and conducting mixed methods research (2nd ed.) SAGE Publications.

Crolley, L., \& Long, C. (2001). Sitting pretty? Women and football in Liverpool. In S. Hopkins, C. Long, \& J. Williams (Eds.) Passing rhythms: Liverpool FC and the transformation of football. (pp. 195-214). Bloomsbury Publishing.

Cronin, C., \& Armour, K. M. (2015). Lived experience and community sport coaching: A phenomenological investigation. Sport, Education and Society, 20(8), 959-975.

Cubitt, S. (1984). Top of the Pops: the Politics of the Living Room. in L. Masterman (ed.) Television Mythologies: Stars, Shows \& Signs. Comedia Publications Group/MK Media Press.

CWHL partners with Senators for Clarkson Cup. (2016, January 26). Vox Media. Retrieved from https://www.pensionplanpuppets.com/2016/1/26/10830622/cwhl-and-ottawa-senatorsannounce-clarkson-cup-partnership

Danermark, B. (2002). Explaining Society: Critical Realism In The Social Sciences. Routledge.

Danford, M., \& Donnelly, P. (2018). 'Racial' Representation in Canadian Interuniversity Sport: A Pilot Study. Toronto: Centre for Sport Policy Studies, Faculty of Kinesiology and Physical Education, University of Toronto. 
D'Arcangelo, L. (2018, May 19). 'There's no break': Overseas double duty is an offer many WNBA stars can't refuse. The Guardian. Retrieved from https://www.theguardian.com/sport/2018/may/19/theres-no-break-overseas-double-dutyis-an-offer-many-wnba-stars-cant-refuse

Das, R. (2019). A field in flux: The intriguing past and the promising future of audience analysis. Television \& New Media, 20(2), 123-129. doi:10.1177/1527476418814592

Dashper, K. (2012). Together, yet still not equal? Sex integration in equestrian sport. AsiaPacific Journal of Health, Sport and Physical Education, 3(3), 213-225.

Data Usage Guidelines. (n.d.). Retrieved from http://en.numeris.ca/data-usage-guidelines

Davidson, J. (2009). Lesbian erotics at women's hockey: Fans, flashing, and the Booby Orrs. Journal of Lesbian Studies, 13(3), 337-348.

Davis, N. W., \& Duncan, M. C. (2006). Sports knowledge is power: Reinforcing masculine privilege through fantasy sport league participation. Journal of Sport and Social Issues, 30(3), 244-264.

Deacon, D. (2007). Researching Communications: A Practical Guide To Methods In Media And Cultural Analysis (2nd ed.) Hodder Arnold.

Dean, J. (2010) Feminism in the papers: Contested feminisms in the British quality press. Feminist Media Studies, 10, 4, 391-407.

Deleuze, G., Guattari, F., \& Massumi, B. (1987). A Thousand Plateaus: Capitalism And Schizophrenia. University of Minnesota Press.

Dominguez, P. (2015). "I'm Very Rich, Bitch!”: The Melodramatic Money Shot and the Excess of Racialized Gendered Affect in the Real Housewives Docusoaps. Camera Obscura: Feminism, Culture, and Media Studies, 30(1 (88)), 155-183.

Donaldson, L., Asbridge, M., \& Cusimano, M. D. (2013). Bodychecking rules and concussion in elite hockey. PloS One, 8(7), e69122-e69122. doi:10.1371/journal.pone.0069122

Donnelly, P. \& M.K. Donnelly (2013). Sex Testing, Naked Inspections and the Olympic GamesA Correction to: The London 2012 Olympics - A Gender Equality Audit. Centre for Sport Policy Studies Research Report. Toronto: Centre for Sport Policy Studies, Faculty of Kinesiology and Physical Education, University of Toronto.

Doyle, G. (2016). Resistance of channels: Television distribution in the multiplatform era. Telematics and Informatics, 33(2), 693-702.

Dreger, A. D. (1998). "Ambiguous Sex"—or Ambivalent Medicine?: Ethical Issues in the Treatment of Intersexuality. Hastings Center Report, 28(3), 24-35.

Dumitriu, D. L. (2014). The mediated live experience and the spatial reconfiguration of the sport act. Revista Română de Comunicare şi Relaţii Publice, 16(3), 35-45. 
Duncan, M. C. (2006). Gender warriors in sport: Women and the media. In A. Raney \& J. Bryant Handbook of sports and media, 231-252. Lawrence Erlbaum Associates.

Duncan, M. C., \& Hasbrook, C. A. (2002). Denial of power in televised women's sports. In S. Scraton \& A. Flintoff, Gender and Sport: A Reader (pp. 83-93). Routledge.

Duncan, M. C., Messner, M. A., Willms, N., Wilson, W., \& Amateur Athletic Foundation of Los Angeles. (2005). Gender In Televised Sports: News And Highlights Shows, 1989-2004. Los Angeles: Amateur Athletic Foundation of Los Angeles.

Dunn, C. (2017). Coming on strong: gender and sexuality in women's sport. Sport in History, 37:2, 258-260, DOI: 10.1080/17460263.2017.1315

Dunning, E. (1986). Sport as a male preserve: Notes on the social sources of masculine identity and its transformations. Theory, Culture \& Society, 3(1), pp. 79-90.

Dworkin, S. L., \& Wachs, F. L. (2009). Body Panic: Gender, Health, And The Selling Of Fitness. NYU Press.

Earle, N. (2002). Hockey as Canadian Popular Culture: Team Canada 1972, Television and the Canadian Identity. Slippery Pastimes: Reading the Popular in Canadian Culture. Wilfred Laurier Press.

Eastman, S. T., \& Billings, A. C. (1999). Gender parity in the Olympics: Hyping women athletes, favouring men athletes. Journal of Sport and Social Issues, 23(2), 140-170.

Eaton, J. (2012). Gender equity in Canadian ice hockey: The legal struggle. Legal Information Management, 12(2), 121-123.

Eco, U. (1990). Towards a semiological guerrilla warfare. Travels in hyper reality: Essays. (1st Harvest/HBJ ed.) Harcourt Brace Jovanovich.

Edwards, L., Jones, C., \& Weaving, C. (2013). Celebration on ice: double standards following the Canadian women's gold medal victory at the 2010 Winter Olympics. Sport in Society, 16(5), 682-698.

Elliott, J. K. (2019, January 29). Humboldt Broncos crash: Global News. What the RCMP forensics team found. Retrieved from https:/globalnews.ca/news/4901621/humboldtbroncos-forensic-report/

Ellis, J. (2000). Seeing things: Television in the age of uncertainty. I.B. Tauris.

Ellison, J. (2002). Women and sport: An examination of advertisements between 1950 and 2002. Canadian Woman Studies, 21(3).

Elueze, R., \& Jones, R. L. (1998). A quest for equality: A gender comparison of the BBC's TV coverage of the 1995 World Athletics Championships. Women in Sport and Physical Activity Journal, 7(1), 45-67. 
Emery, C. A., Kang, J., Shrier, I., Goulet, C., Hagel, B. E., Benson, B. W., Nettel-Aguirre, A., McAllister, J.R., Hamilton, G.M., \& Meeuwisse, W. H. (2010). Risk of injury associated with bodychecking among youth ice hockey players. Jama, 303(22), 2265-2272

Enli, G., \& Syvertsen, T. (2016). The end of Television-Again! how TV is still influenced by cultural factors in the age of digital intermediaries. Media and Communication, 4(3), 142153. doi:10.17645/mac.v4i3.547

Esmonde, K., Cooky, C., \& Andrews, D. L. (2018). "That's not the only reason I'm watching the game": Women's (hetero)sexual desire and sports fandom. Journal of Sport \& Social Issues, 42(6), 498-518. doi:10.1177/0193723518797041

Etue, E., \& Williams, M. K. (1996). On The Edge: Women Making Hockey History. Second Story Press.

Everbach, T. (2018). "I Realized It Was About Them... Not Me": Women Sports Journalists and Harassment. In J.R. Vickery and T. Everbach, Mediating Misogyny (pp. 131-149). Palgrave Macmillan, Cham.

Farred, G. (2018). The Burden Of Over-representation: Race, Sport, And Philosophy. Temple University Press.

Farrell, A. O. (2006). Why Women Don't Watch Women's Sport: A Qualitative Analysis (Doctoral Dissertation). The Ohio State University).

Farrell, A., Fink, J. S., \& Fields, S. (2011). Women's sport spectatorship: An exploration of men's influence. Journal of Sport Management, 25(3), 190-201.

Fausto-Sterling, A. (2000). Sexing the body: Gender politics and the construction of sexuality. Basic Books.

Featherstone. L. (2018). Divining Desire: Focus Groups and the Culture of Consultation. OR Books: London.

Feeley, B. T., Agel, J., \& LaPrade, R. F. (2016). When is it too early for single sport specialization?. The American journal of sports medicine, 44(1), 234-241.

FIFA.com. (2015, December 17). FIFA Women's World Cup 2015 FIFA Women's World Cup tops 750 million TV viewers. Retrieved from https://www.fifa.com/womensworldcup/news/record-breaking-fifa-women-s-world-cuptops-750-million-tv-viewers-2745963

Fink, J. S. (2012). Homophobia and the marketing of female athletes and women's sport. In G.B. Cunningham Sexual Orientation And Gender Identity In Sport: Essays From Activists, Coaches, And Scholars (pp. 49-60). Center for Sport Management Research and Education, Texas A \& M University.

Fink, J. S. (2015). Female athletes, women's sport, and the sport media commercial complex: Have we really "come a long way, baby"? Sport management review, 18(3), 331-342. 
Fiske, J. (1985). The semiotics of television. Critical Studies in Mass Communication, 2(2), 176183.

Fiske, J. (1987). Television culture. Routledge.

Fiske, J. (1990). Ethnography and everyday life. Routledge.

Fiske, J. (1992). Audiencing: A cultural studies approach to watching television. Poetics, 21(4), 345-359. doi:10.1016/0304-422X(92)90013-S

Fiske, J. (2001). Reading the popular. Taylor \& Francis e-Library.

Flames partner with CWHL to support Team Alberta. (2012, November 13). [Press Release] Retrieved from https://www.nhl.com/news/flames-partner-with-cwhl-to-support-teamalberta/c-645586

Foddy, B., \& Savulescu, J. (2011). Time to re-evaluate gender segregation in athletics?. British Journal of Sports Medicine, 45(15), 1184-1188.

Fortier-Brynaert, E. (2011). 'She Shoots, She Scares"! Online Reader Responses and the Backlash Against the 2010 Canadian Olympic Women's Hockey Team on Ice Celebration. [Masters Major Research Project] University of Ottawa.

Foucault, M. (1981). History of systems of thought. Philosophy \& Social Criticism, 8(3), 353359.

Francombe, J. (2010). "I cheer, you cheer, we cheer": Physical technologies and the normalized body. Television \& New Media, 11(5), 350-366.

Fraser, N. (1985). What's critical about critical theory? The case of Habermas and gender. New German Critique, (35), 97-131.

Freud, S. (1940). An Outline Of Psychoanalysis. Standard Edition 23.

Frey, W. H. \& Davis, K. (2018). Shifting Demographics And Hockey's Future [Policy Brief]. NHL Social Impact, Growth Initiatives \& Legislative Affairs Lecture Series. Retrieved from https://nhl.bamcontent.com/images/assets/binary/300993502/binary-file/file.pdf

Friedman, E. (2017, April 04). NHL players won't be at the 2018 Olympics, and that's bad for hockey. MacLeans Magazine. Retrieved from https://www.macleans.ca/sports/nhlplayers-wont-be-at-the-2018-olympics-and-thats-bad-for-hockey/

Friedman, E. (2019, April 2). Thoughts: NHL-backed women's league the 'obvious solution'. Sportsnet. Retrieved from https://www.sportsnet.ca/hockey/nhl/31-thoughts-nhl-backedwomens-league-obvious-solution/

Frisk, A. (2018, April 10). Canadian soldiers in Iraq put their hockey sticks out for Humboldt Broncos. Global News. Retrieved from https://globalnews.ca/news/4135173/humboldtbroncos-hockey-sticks-out-canadian-soldiers-iraq/ 
Francombe-Webb, J., \& Fullagar, S. (2015, January 16). The This Girl Can campaign is all about sex, not sport | Simone Fullagar. Retrieved from https://www.theguardian.com/commentisfree/2015/jan/16/this-girl-can-campaign-sexsport-real-women-bodies-objectifying-female-flesh

Gall, D. (2016). Weight of the Pass. Unpublished manuscript.

Gall, D. (Producer/Director), McIvor, C. (Producer), Peeler, J. (Producer). (2015) Play Fair. Canada: Frank Digital/Frantic Films.

Gamson, W. A. (1992). Talking Politics Cambridge University Press.

Gantz, W., \& Wenner, L. A. (1991). Men, women, and sports: Audience experiences and effects. Journal of Broadcasting \& Electronic Media, 35(2), 233-243.

Gantz, W., Wang, Z., Paul, B., \& Potter, R. F. (2006). Sports versus all comers: Comparing TV sports fans with fans of other programming genres. Journal of Broadcasting \& Electronic Media, 50(1), 95-118. doi:10.1207/s15506878jobem5001_6

Gauntlett, D. (2007). Creative explorations: New approaches to identities and audiences. Routledge.

Gee, S. (2009). Mediating sport, myth, and masculinity: The National Hockey League's "Inside the Warrior" advertising campaign. Sociology of Sport Journal, 26(4), 578-598.

Geraghty, C. (1991). Women and soap opera: A study of prime time soaps. Polity Press.

Gibbs, A. (2011). Affect theory and audience. In V. Nightingale, The Handbook Of Media Audiences (pp. 251-266). Wiley-Blackwell.

Gilenstam, K. (2009). Gender And Physiology In Ice Hockey: A Multidimensional Study [Doctoral dissertation], Umeå University.

Gill, R. (2007) Gender and the Media. Cambridge: Polity Press.

Gill, R. (2007). Postfeminist media culture: Elements of a sensibility. European journal of cultural studies, 10(2), 147-166.

Gill, R. (2008). Culture and subjectivity in neoliberal and postfeminist times. Subjectivity, 25(1), 432-445.

Gillespie, M. (1995). Television, ethnicity, and cultural change. Routledge.

Gillis, S. and Munford, R. (2004) Genealogies and generations: The politics and praxis of third wave feminism. Women's History Review, 13, 2, 165-82.

Ginés, I. C. (2014). Blood Ties: Interpretive Communities and Popular (Gendered) Genres. Critical Studies, 37, 233.

Gitlin, T. (1978). Media sociology: The dominant paradigm. Theory and Society, 6(2), 205-253. 
Glaser, B.G. (2008). Qualitative and quantitative research. Grounded Theory Review: An International Journal, 7(2). 99-113.

Glaser, B. G., \& Strauss, A. L. (1967). The discovery of grounded theory: Strategies for qualitative research. Aldine Pub. Co.

Goetz, A. M. (2007). Political cleaners: Women as the new anti-corruption force?. Development and Change, 38(1), 87-105.

Goldberg, M. E., Sandikci, O., \& Litvack, D. (2018). Reducing the level of violence in hockey. In M. E. Goldberg \& M. Fishbein (Eds.), Social Marketing (pp. 335-352). Psychology Press.

Gordon, S. (2016, November 18). NWHL cuts salaries to save season. Sports Illustrated. Retrieved from https://www.si.com/2016/11/18/ap-hko-womens-league-pay-cuts-1st-ldwritethru

Gorton, K. (2009). Media Audiences: Television, Meaning And Emotion. Edinburgh University Press.

Gosling, V.K. (2007) 'Girls Allowed? The Marginalization of Female Sport fans' in J. Gray, C. Sandvoss, and C. L. Harrington (Eds.), Fandom: Identities and Communities in a Mediated World, (pp. 250-260). New York University Press.

Grainger, A., Newman, J., \& Andrews, D. (2006). Sport, the media and the construction of race. In A. Raney \& J. Bryant (Eds.), Handbook Of Sports And Media (pp. 447-468). Routledge.

Gray, A. (2006). Video Playtime: The Gendering Of A Leisure Technology. Routledge.

Gray, J. (2017). Reviving audience studies. Critical Studies in Media Communication, 34(1), 7983.

Gray, J., \& Lotz, A. D. (2012). Television Studies Polity Press.

Gray, J., Sandvoss, C., \& Harrington, L. (2007). Why study fans?. New York University Press.

Greene, J. C. (2007). Mixed Methods In Social Inquiry (Vol. 9). John Wiley \& Sons.

Greene, L. S. (2016). Mirror, mirror on the wall - gender, Olympic competition and persistence of the feminine ideal. Wisconsin Journal of Law, Gender \& Society, 31(2), 57.

Gregg, M., \& Seigworth, G. J. (Eds.). (2010). The Affect Theory Reader. Duke University Press.

Griffin, P. (1998). Strong Women, Deep Closets: Lesbians And Homophobia In Sport. Human Kinetics Publishers.

Grindstaff, L. (2002). The Money Shot: Trash, Class, And The Making Of TV Talk Shows. University of Chicago Press. 
Grossberg, L. (1997). Bringing It All Back Home: Essays On Cultural Studies. Duke University Press.

Gruneau, R. S., \& Whitson, D. (2006). Artificial ice: Hockey, culture, and commerce. University of Toronto Press.

Gruneau, R., \& Whitson, D. (1993). Hockey Night In Canada: Sport, Identities And Cultural Politics. Garamond Press.

Guba, E. G., \& Lincoln, Y. S. (1989). Fourth generation evaluation. Sage.

Guba, E. G., \& Lincoln, Y. S. (2005). Paradigmatic Controversies, Contradictions, and Emerging Confluences. In N. K. Denzin \& Y. S. Lincoln (Eds.), The Sage Handbook Of Qualitative Research (pp. 191-215). Sage Publications Ltd.

Guest, A. M., \& Luijten, A. (2018). Fan culture and motivation in the context of successful women's professional team sports: a mixed-methods case study of Portland Thorns fandom. Sport in Society, 21(7), 1013-1030.

Guetterman, T. C., Babchuk, W. A., Howell Smith, M. C., \& Stevens, J. (2019). Contemporary approaches to mixed methods-grounded theory research: A field-based analysis. Journal of Mixed Methods Research, 13(2), 179-195

Gunter, B. (2000). Media Research Methods: Measuring Audiences, Reactions And Impact. Sage.

Gunter, B., \& Svennevig, M. (1987). Behind And In Front Of The Screen: Television's Involvement With Family Life. John Libbey \& Company.

Hall, A. (2009). Perceptions of the authenticity of reality programs and their relationships to audience involvement, enjoyment, and perceived learning. Journal of Broadcasting \& Electronic Media, 53(4), 515-531.

Hall, C.M. and Hodges, J. (1996) 'The party's great, but what about the hangover? The housing and social impacts of mega-events with special reference to the 2000 Sydney Olympics', Festival Management and Event Tourism, 4(1), 13-20.

Hall, M. A. (2002). The girl and the game: A history of women's sport in Canada Broadview Press.

Hall, M. A. (2013). Toward a history of Aboriginal women in Canadian sport. Aboriginal Peoples \& Sport In Canada: Historical Foundations And Contemporary Issues, 64-91.

Hall, M. A. (2016). The girl and the game: A history of women's sport in Canada (Second ed.) University of Toronto Press.

Hall, S. (1980). Culture, Media, Language: Working Papers In Cultural Studies, 1972-79. Routledge in association with the Centre for Contemporary Cultural Studies, University of Birmingham. 
Hankivsky, O., \& Grace, D. (2015). Understanding and emphasizing difference and intersectionality in multimethod and mixed methods research. In S.N. Hesse-Biber and R. B. Johnson (Eds.), The Oxford Handbook Of Multimethod And Mixed Methods Research Inquiry. Oxford University Press.

Hardin, M., \& Greer, J. D. (2009). The influence of gender-role socialization, media use and sports participation on perceptions of gender-appropriate sports. Journal of Sport Behaviour, 32(2), 207.

Hardin, M., \& Whiteside, E. (2012). How do women talk sports? Women sports fans in a blog community. In Toffoletti \& Mewett (Eds.), Sport And Its Female Fans, 152-168. Routledge.

Hardin, M., Chance, J., Dodd, J. E., \& Hardin, B. (2002). Olympic photo coverage fair to female athletes. Newspaper Research Journal, 23(2-3), 64-78.

Hargreaves, J. (1985). The body, sport and power relations. The Sociological Review, 33(S1), pp. 139-159.

Hargreaves, J. (1994). Sporting Females: Critical Issues In The History And Sociology Of Women's Sports. Routledge.

Hargreaves, J., \& Anderson, E. (Eds.). (2014). Routledge Handbook Of Sport, Gender And Sexuality. Routledge.

Haring, B. (2018, September 09). Serena Williams Meltdown Leads To US Open Tennis Final Loss, A Major Upset. Deadline. Retrieved from https://deadline.com/2018/09/serenawilliams-meltdown-leads-to-us-open-tennis-final-loss-a-major-upset-1202460377/

Harris, A. (2004). Future Girl: Young Women In The Twenty-first Century. Routledge.

Harris, J., \& Clayton, B. (2002). Femininity, masculinity, physicality and the English tabloid press: The case of Anna Kournikova. International Review For The Sociology Of Sport, 37(3-4), 397-413.

Harrison, R., \& Dopp, J. (Eds.). (2009). Now Is the Winter: Thinking about Hockey. Wolsak and Wynn.

Harry, J. (1995). Sports ideology, attitudes toward women, and anti-homosexual attitudes. Sex Roles, 32(1-2), 109-116.

Hartmann, H. (1976). Capitalism, patriarchy, and job segregation by sex. Signs: Journal of Women in Culture and Society, 1(3, Part 2), 137-169.

Harvey, J. (2006). Whose Sweater Is This The Changing Meanings of Hockey in Quebec. In David Whitson \& Richard Gruneau (Eds.), Artificial Ice: Hockey, culture, and commerce. University of Toronto Press. 29-52.

Hayhurst, L. M. (2014). The 'girl effect' and martial arts: Social entrepreneurship and sport, gender and development in Uganda. Gender, place \& culture, 21(3), 297-315. 
Hayhurst, L. M., Kay, T., \& Chawansky, M. (Eds.). (2015). Beyond Sport For Development And Peace: Transnational Perspectives On Theory, Policy And Practice. Routledge.

Heinecken, D. (2015). Pretty tough sports and the promotion of female empowerment in young adult sports fiction. The Lion and the Unicorn, 39(1), 23-41.

Heinecken, D. (2016). Gender and jockography: post-feminism and resistance in female sports autobiographies. Feminist Media Studies, 16(2), 325-343.

Hermes, J. O. (2014). Rediscovering twentieth-century feminist audience research. In C. Carter, L. Steiner, \& L. McLaughlin. The Routledge Companion to Media \& Gender (pp. 79-88). Routledge.

Herzog, H. (1941). On borrowed experience: An analysis of listening to daytime sketches. Zeitschrift für Sozialforschung, 9(1), 65-95.

Herzog, H. (1944). What do we really know about daytime serial listeners? In P.F. Lazarsfeld \& F.N. Stanton (Eds.), Radio Research 1942-1943. (pp. 3-33). New York: Duell, Sloan \& Pearce.

Hesse-Biber, S. N. (2010). Mixed Methods Research: Merging Theory With Practice. Guilford Press.

Hesse, M. (2019, May 02). We celebrated Michael Phelps's genetic differences. Why punish Caster Semenya for hers? The Washington Post. Retrieved May 10, 2019, from https://www.washingtonpost.com/lifestyle/style/we-celebrated-michael-phelpss-geneticdifferences-why-punish-caster-semenya-for-hers/2019/05/02/93d08c8c-6c2b-11e9-be3a33217240a539_story.html?utm_term=.28743df4aacc

Hewings, M. (2006, December 6). Sharing the Hockey Dream. The Montreal Gazette. Retrieved from https://www.pressreader.com/canada/montrealgazette/20061216/281960308265011

Hill, A. (2004). Reality TV: Factual Entertainment And Television Audiences. Routledge.

Hills, L., \& Kennedy, E. (2009). Double trouble: Kelly Holmes, intersectionality and unstable narratives of Olympic heroism in the British media. In P. Markula (Ed.), Olympic Women And The Media (pp. 112-131). Palgrave Macmillan, London.

Hirdman, A. (2016). The passion of mediated shame: Affective reactivity and classed otherness in reality TV. European Journal Of Cultural Studies, 19(3), 283-296.

Hobson, D. (1982). Crossroads: The Drama Of A Soap Opera. Methuen Limited.

Hobson, W. (2018). At Larry Nassar sentencing hearing, a parade of horror and catharsis. The Washington Post. Retrieved April 20, 2019, from https://www.washingtonpost.com/sports/olympics/at-larry-nassar-sentencing-hearing-aparade-of-horror-and-catharsis/2018/01/18/19bed832-fc55-11e7-8f662df0b94bb98a_story.html?utm_term=.e558d73a1f68 
Hockey Night in Canada salutes Gord Downie. (2016, May 25). Sportsnet. Retrieved March 25, 2019, from https://www.sportsnet.ca/hockey/nhl/hockey-nigh-canada-salutes-gorddownie/

Hoeber, L., \& Kerwin, S. (2013). Exploring the experiences of female sport fans: A collaborative self-ethnography. Sport Management Review, 16(3), 326-336.

Hoffman, A., \& Meixler, E. (2018, February 22). Canadian Jocelyn Larocque Takes off Silver Hockey Medal. Time Magazine. Retrieved from https:/time.com/5170424/canadiantakes-off-medal-olympics/

Höijer, B. (2008). Ontological assumptions and generalizations in qualitative (audience) research. European Journal of Communication, 23(3), 275-294.

Horkheimer, M., \& Adorno, T. W. (1972). Dialectic Of Enlightenment. Herder and Herder.

Horning, D. L. (2018). Women's Recreational Hockey: A New Player Profile. In S. Irving, N. Baillargeon, \& C. Boissinot, (Eds.). Hockey: Challenging Canada's Game = Au-delà du Sport National (pp. 165 - 178). University of Ottawa Press.

Houghton, C., Murphy, K., Meehan, B., Thomas, J., Brooker, D., \& Casey, D. (2017). From screening to synthesis: using NVivo to enhance transparency in qualitative evidence synthesis. Journal Of Clinical Nursing, 26(5-6), 873-881.

Hovland, C. I. (1951). Changes in attitude through communication. The Journal of Abnormal and Social Psychology, 46(3), 424-437. http://dx.doi.org/10.1037/h0055656

Huiberts, E. (2016). Building bridges, filling gaps: Toward an integrative interdisciplinary and mixed method approach for future audience research in relation to the mediation of distant suffering. International Journal of Communication, 10, 21.

Hutchins, A. (2017a, December 06). Olympic hockey without NHL players will be a can-miss event. MacLeans Magazine. Retrieved from https:/www.macleans.ca/sports/olympichockey-without-nhl-players-will-be-a-can-miss-event/

Hutchins, A. (2017b, November 10). Why Harrison Browne couldn't retire from women's hockey. MacLeans Magazine. Retrieved from https://www.macleans.ca/news/canada/why-harrison-browne-couldnt-retire-fromwomens-hockey/

Hutchins, B., \& Rowe, D. (2012). Sport Beyond Television: The Internet, Digital Media And The Rise Of Networked Media Sport. Routledge.

Hynes, D., \& Cook, A. M. (2013). Online belongings: Female fan experiences in online soccer forums. In B. Hutchins \& D. Rowe (Eds.), Digital Media Sport (pp. 115-128). Routledge.

Ingle, S. (2019, May 01). Caster Semenya loses landmark legal case against IAAF over testosterone levels. The Guardian. Retrieved from https://www.theguardian.com/sport/2019/may/01/caster-semenya-loses-landmark-legalcase-iaaf-athletics 
Interim CWHL commissioner defends decision to fold league. (2019, April 02). CBC News. Retrieved from https://www.cbc.ca/news/canada/ottawa/cwhl-shutting-down-jaynahefford-1.5080831

Itani, S. (2015). Japanese Female and 'Trans' Athletes: Negotiating Subjectivity and Media Constructions of Gender, Sexuality, and Nation. University of Toronto (Canada).

James, A. (2013). Seeking the analytic imagination: reflections on the process of interpreting qualitative data. Qualitative Research, 13(5), 562-577.

James, W. (1952). Principles Of Psychology. Encyclopaedia Britannica.

Jameson, F. (1991). Postmodernism, Or, The Cultural Logic Of Late Capitalism. Duke University Press.

Jamieson, S. (2014). Reading the "St. Louis Whirligig": Hockey, Masculinity, and Aging in Paul Quarrington's King Leary. Journal of Canadian Studies, 48(3), 181-199.

Jenkins, H. (1992). Textual poachers: Television fans \& participatory culture Routledge.

Jenkins, H. (2012). Never Trust a Snake: WWF Wrestling as Masculine Melodrama. In MC Kearney (Ed.) The Gender and Media Reader. Routledge.

Jhally, S. (1982). Probing the blindspot: The audience commodity. Canadian Journal of Political and Social Theory, 6(1-2), 204-210.

Jhally, S. (1984). The spectacle of accumulation: Material and cultural factors in the evolution of the Sports/Media complex. The Insurgent Sociologist, 12(3), 41.

Jhally, S., \& Lewis, J. (1992). Enlightened Racism: The Cosby Show, Audiences, And The Myth Of The American Dream. Westview Press.

Lewis, J., \& Jhally, S. (1994). The Politics of Cultural Studies: Racism, Hegemony, and Resistance. American Quarterly, 46(1), 114-117. doi:10.2307/2713361

Jhally, S., \& Livant, B. (1986). Watching as working: The valorization of audience consciousness. Journal of Communication, 36(3), 124-143.

Johnstone, L. \& Millar, S. (2012). Actively Engaging Women and Girls: Addressing the PsychoSocial Factors. Canadian Association for the Advancement of Women in Sport. Retrieved from http://www.caaws.ca/ActivelyEngaging/documents/CAAWS_CS4L_Engaging_Women. pdf

Jones, K. W. (2008). Female fandom: Identity, sexism, and men's professional football in England. Sociology of Sport Journal, 25(4), 516-537.

Jones, R., Murrell, A. J., \& Jackson, J. (1999). Pretty versus powerful in the sports pages: Print media coverage of US women's Olympic gold medal winning teams. Journal of Sport and Social Issues, 23(2), 183-192. 
Kaillie Humphries files harassment complaint with Bobsleigh Canada. (2019, January 21). CBC Sports. Retrieved from https://www.cbc.ca/sports/olympics/bobsleigh/kaillie-humphriesbobsleigh-canada-harassment-1.4985486

Kamberelis, G., \& Dimitriadis, G. (2013). Focus Groups. London: Routledge.

Kane, M. J. (1995). Resistance/transformation of the oppositional binary: Exposing sport as a continuum. Journal Of Sport And Social Issues, 19(2), 191-218.

Kane, M. J. (1996). Media coverage of the post Title IX female athlete: A feminist analysis of sport, gender, and power. Duke Journal of Gender Law \& Policy, 3, 95.

Kane, M. J. (2013). The better sportswomen get, the more the media ignore them. Communication \& Sport, 1(3), 231-236. doi:10.1177/2167479513484579

Kane, M. J., \& Disch, L. J. (1993). Sexual violence and the reproduction of male power in the locker room: The "Lisa Olson incident". Sociology of Sport Journal, 10(4), 331-352.

Kane, M. J., \& Maxwell, H. D. (2011). Expanding the boundaries of sport media research: Using critical theory to explore consumer responses to representations of women's sports. Journal of Sport Management, 25(3), 202-216.

Kane, M. J., LaVoi, N. M., \& Fink, J. S. (2013). Exploring elite female athletes' interpretations of sport media images: A window into the construction of social identity and "selling sex” in women's sports. Communication \& Sport, 2167479512473585.

Kaplan, E. (2019, May 17). Devils ending partnership with NWHL's Riveters. ESPN. Retrieved from https://www.espn.co.uk/nhl/story/_id/26768491/devils-ending-partnership-nwhlriveters

Karlyn, K.R. (2003). Scream, Popular Culture, and Feminism's Third Wave: 'I'm Not My Mother'. Genders, Volume 38

Katz, E. (1959). Mass communications research and the study of popular culture: An editorial note on a possible future for this journal. Departmental Papers (ASC), 165.

Katz, E., \& Lazarsfeld, P. F. (2006). Personal influence: The part played by people in the flow of mass communications (2nd ed.). Transaction Publishers.

Katz, E., \& Scannell, P. (2009). The end of television? It's impact on the world (so far)'. Special edition of The Annals of the American Academy of Political and Social Science, 625.

Katz, E., Blumler, J. G., \& Gurevitch, M. (1973). Uses and gratifications research. The Public Opinion Quarterly, 37(4), 509-523.

Kaufman, P., \& Wolff, E. A. (2010). Playing and protesting: Sport as a vehicle for social change. Journal of Sport and Social Issues, 34(2), pp. 154-175.

Kavka, M. (2008). Reality television, affect and intimacy: Reality matters: Palgrave Macmillan. 
Kellner, D. (2011). Cultural studies, multiculturalism, and media culture. Gender, Race, And Class In Media: A Critical Reader, 3, 7-18.

Kennedy, E., \& Hills, L. (2015). Sport, Media And Society. Bloomsbury Publishing.

Kenny, A. J. (2005). Interaction in cyberspace: an online focus group. Journal of Advanced Nursing, 49(4), 414-422.

Kessel, A. (2018, September 09). Serena Williams again bears brunt of double standards in tennis | Anna Kessel. The Guardian. Retrieved from https://www.theguardian.com/sport/2018/sep/09/serena-williams-again-bears-bruntdouble-standards-tennis

Kidd, B. (1990). The men's cultural centre. In M.A. Messner \& D.F. Sabo (Eds.), Sport, Men, And The Gender Order: Critical Feminist Perspectives, (pp. 31-43). Human Kinetics Books.

Kidd, B. (2013). Canada's 'national' sport. Sport in Society, 16(4), 351-361. doi:10.1080/17430437.2013.785747

Kidd, B. (2018). Towards responsible policy-making in international sport: Reforming the medical-scientific commissions. Sport in Society, 21(5), 773-787.

Kim, K., Cheong, Y., \& Kim, H. (2016). Competition and coexistence of sports media: The case of watching the 2014 Sochi Winter Olympic Games. Asian Journal of Communication, 26(5), 485-503.

Kimble, C. E., \& Cooper, B. P. (1992). Association and dissociation by football fans. Perceptual and Motor Skills, 75(1), 303-309.

King, S. (1993). The politics of the body and the body politic: Magic Johnson and the ideology of AIDS. Sociology of Sport Journal, 10(3), 270-285.

Kinnick, K. N. (1998). Gender bias in newspaper profiles of 1996 Olympic athletes: A content analysis of five major dailies. Women's Studies in Communication, 21(2), 212-237.

Kirby Letts, G., \& Steckley, J. (2010). Social inequality. In J. Steckley \& G.K. Letts (Eds.), Elements Of Sociology: A Critical Canadian Introduction (2nd ed.). Oxford University Press.

Kissane, R. J., \& Winslow, S. (2016). "You're Underestimating Me and You Shouldn't" Women's Agency in Fantasy Sports. Gender \& Society, 30(5), 819-841.

Kite, J., \& Phongsavan, P. (2017). Insights for conducting real-time focus groups online using a web conferencing service. F1000research, 6, 122. doi:10.12688/f1000research.10427.1

Kitzinger, J. (1994). The methodology of focus groups: the importance of interaction between research participants. Sociology of health \& illness, 16(1), 103-121.

Kniffin, K. M., \& Palacio, D. (2018). Trash-talking and trolling. Human Nature, 29(3), 353-369. Hawthorne. doi:10.1007/s12110-018-9317-3 
Knight, D. (1999). Why we enjoy condemning sentimentality: A meta-aesthetic perspective. The Journal of Aesthetics and Art Criticism, 57(4), 411-420.

Knight, H. [@HilaryKnight]. (2019, May 2). We may represent different teams, leagues and countries but collectively we stand as one. [Tweet]. //twitter.com/HilaryKnight/status/1123965390747525121/photo/1?ref_src=twsrc\%5Etfw $\%$ 7Ctwcamp \%5Etweetembed\%7Ctwterm\%5E1123965390747525121\&ref_url=https\%3 A\%2F\%2Fwww.npr.org\%2F2019\%2F05\%2F02\%2F719627264\%2F200-female-prohockey-players-lay-down-their-sticks-demanding-better-conditions

Knobloch-Westerwick, S., David, P., Eastin, M. S., Tamborini, R., \& Greenwood, D. (2009). Sports spectators' suspense: Affect and uncertainty in sports entertainment. Journal of Communication, 59(4), 750-767.

Koivula, N. (1999). Gender stereotyping in televised media sport coverage. Sex roles, 41(7-8), 589-604.

Krane, V. (1998). Lesbians in sport: Toward acknowledgment, understanding, and theory. Journal of sport and exercise psychology, 20(3), 237-246.

Krane, V. (2001). We can be athletic and feminine, but do we want to? Challenging hegemonic femininity in women's sport. Quest, 53(1), 115-133.

Krebs, A. (2012). Hockey and the reproduction of colonialism in Canada. In J. Joseph, S. Darnell, \& Y. Nakamura (Eds.). Race and sport in Canada: Intersecting inequalities. Canadian Scholars Press.

Lafrance, M. R. (1998). Colonizing the feminine: Nike's intersections of postfeminism and hyperconsumption. In G. Rail (Ed.), Sport And Postmodern Times, 117-39. SUNY Press.

Langley, S. (2003). Gender, talk, TV, hockey, and "Canadian identity": Feminist takes on "television rejection". Canadian Journal of Communication, 28(4), 413. doi:10.22230/cjc.2003v28n4a1390

Larkin, M. (2015, January 05). The fight to sell women's hockey: Can the CWHL become the WNBA? The Hockey News. Retrieved from https://thehockeynews.com/news/article/thefight-to-sell-womens-hockey-can-the-cwhl-become-the-wnba

Lasswell, H. D. (1927). Propaganda technique in the world war. Ravenio Books.

Latour, B. (1994). Pragmatogonies. A mythical account of how humans and non-humans swap properties. American Behavioural Scientist, 37(6), 791-808.

Lavelle KL. As Venus Turns: A Feminist Soap Opera Analysis of Venus Vs. Journal of Sports Media. 2015;10:1-16.

Lawson, B. (2004). What Designers Know. Routledge.

Lazarsfeld, P. F. (1941). Remarks on administrative and critical communications research. Zeitschrift für Sozialforschung, 9(1), 2-16. 
Lazarsfeld, P. F. (1948). The use of panels in social research. Proceedings of the American Philosophical Society, 92(5), 405-410.

Lazarsfeld, P. F., \& Dinerman, H. (1949). Research for Action. In Lazarsfeld, P. F., \& Stanton, F. N. (Eds.). Communications Research 1948-1949. Harper \& Brothers: New York.

Lazarsfeld, P. F., Gaudet, H., \& Berelson, B. (1968). People's Choice: How The Voter Makes Up His Mind In A Presidential Campaign (3rd ed.) Columbia University Press.

Lazzarato, M. (2006). The Concepts of Life and the Living in the Societies of Control. In M. Fuglsang \& S. Meier (Eds.), Deleuze and the Social (171-190). Edinburgh University Press.

Leafs Announce Partnership Agreement with Toronto Furies. (2012, November 13). [Press Release]. Retrieved from https://www.nhl.com/mapleleafs/news/leafs-announcepartnership-agreement-with-toronto-furies/c-645484

Lee, R. (2019). An Economic Evaluation of Bodychecking Policies in Bantam Ice Hockey [Master's thesis]. Cumming School of Medicine.

Lee, R. M., \& Fielding, N. G. (1991). Computing for qualitative research: Options, problems and potential. In N. G. Fielding \& R. M. Lee (Eds.), Using Computers In Qualitative Research (pp. 1-13). Sage.

Legislative Services Branch. (1994). Consolidated federal laws of Canada, National Sports of Canada Act. Retrieved from https://laws-lois.justice.gc.ca/eng/acts/n-16.7/page-1.html

Lenskyj, H. (2012). Best Olympics Ever? The Social Impacts of Sydney 2000. SUNY Press.

Lenskyj, H., \& Sport Canada. (1994). Women, Sport, And Physical Activity: Selected Research Themes. Sport Information Resource Centre for Sport Canada.

Lever, J., \& Wheeler, S. (1993). Mass media and the experience of sport. Communication Research, 20(1), 125-143.

Levy, Y. (2018). Serial housewives: the feminist resistance of The Real Housewives' matrixial structure. Continuum, 32(3), 370-380.

Lewis, J. (1991). The Ideological Octopus: An Exploration Of Television And Its Audience. Routledge .

Lewis, P. (2014) Postfeminism, Femininities and Organization Studies: Exploring a New Agenda. Organization Studies, 35, 12, 1845-66

Lindner, K. (2012). Women's Boxing at the 2012 Olympics: Gender trouble?. Feminist Media Studies, 12(3), 464-467.

Lines, G. (2004). The sports star in the media: the gendered construction and youthful consumption of sports personalities. In L. Misener (Ed.), Power games: A critical sociology of sport (pp. 196-215). Human Kinetics Publishers, Inc. 
Lippmann, W. (2017). Public Opinion. Routledge.

Livingstone, S. M. (1993). The rise and fall of audience research: An old story with a new ending. Journal of Communication, 43(4), 5-12. doi:10.1111/j.1460-2466.1993.tb01298.x

Livingstone, S. (1998). Audience research at the crossroads: the 'implied audience' in media and cultural theory. European journal of cultural studies, 1(2), 193-217.

Livingstone, S. (2003). The changing nature of audiences. A companion to media studies, 6, 337.

Livingstone, S. (2004). The challenge of changing audiences: Or, what is the audience researcher to do in the age of the Internet? European journal of communication, 19(1), 75-86.

Livingstone, S. (2008). Audiences and interpretations. E-Compós, 10doi:10.30962/ec.v10i0.185

Livingstone, S. (2013). The participation paradigm in audience research. The Communication Review, 16(1-2), 21-30.

Livingstone, S. (2019). Audiences in an age of datafication: Critical questions for media research. Television \& New Media, 20(2), 170-183. doi:10.1177/1527476418811118

Livingstone, S., \& Das, R. (2013). The end of audiences? In J. Hartley, J.E. Burgess, \& A. Bruns (Eds.), A Companion To New Media Dynamics (pp. 104-122). Chichester and Malden.

Loiperdinger, M., \& Elzer, B. (2004). Lumière's arrival of the train: Cinema's founding myth. The Moving Image, 4(1), 89-118.

Longino, H. E., \& Miner, V. (Eds.). (1987). Competition, a Feminist Taboo?. Feminist Press at the City University of New York.

Longley, R. (2018a, February 23). Dark day for Canadian hockey: Men's Olympic team loses to Germany in semifinals. The National Post. Retrieved from https://nationalpost.com/sports/olympics/olympic-shocker-germany-upsets-canada-inmens-hockey-semifinal

Longley, R. (2018b, February 23). Jocelyne Larocque sorry for taking off silver medal after women's hockey loss. Retrieved from https://nationalpost.com/sports/olympics/jocelynelarocque-sorry-for-taking-off-silver-medal-after-womens-hockey-loss

Lorenz, S. L. (2015). Constructing a cultural history of Canadian hockey. The International Journal of the History of Sport, 32(17), 2107-2113. doi:10.1080/09523367.2016.1152265

Love, A., \& Kelly, K. (2011). Equity or essentialism? US courts and the legitimation of girls' teams in high school sport. Gender \& Society, 25(2), 227-249.

Lucas-Carr, C. B., \& Krane, V. (2011). What is the T in LGBT? Supporting transgender athletes through sport psychology. The Sport Psychologist, 25(4), 532-548.

Lucas-Carr, C., \& Krane, V. (2012). Troubling sport or troubled by sport. Journal for the Study of Sports and Athletes in Education, 6(1), 21-44. 
Lucas, K., \& Sherry, J. L. (2004). Sex differences in video game play: A communication-based explanation. Communication Research, 31(5), 499-523.

Lunt, P., \& Livingstone, S. (1996). Rethinking the focus group in media and communications research. Journal of Communication, 46(2), 79-98.

MacNeill, M. (1996). Networks: Producing Olympic ice hockey for a national television audience. Sociology of Sport Journal, 13(2), 103-124.

MacNeill, M. (2009). Opening up the gendered gaze: Sport media representations of women, national identity and the racialised gaze in Canada. In Markula, P. (Ed.), Olympic Women and the Media (pp. 50-69). Palgrave Macmillan.

Macpherson, A., Rothman, L., \& Howard, A. (2006). Body-checking rules and childhood injuries in ice hockey. Paediatrics, 117(2), e143-e147.

Macur, J. (2017, August 04). What Qualifies a Woman to Compete as a Woman? An Ugly Fight Resumes. The New York Times. Retrieved from https://www.nytimes.com/2017/08/04/sports/olympics/gender-dutee-chand-india.html

Madrigal, A. C. (2018, May 30). When Did TV Watching Peak? The Atlantic. Retrieved from https://www.theatlantic.com/technology/archive/2018/05/when-did-tv-watchingpeak/561464/

Maher, C., Hadfield, M., Hutchings, M., \& de Eyto, A. (2018). Ensuring rigor in qualitative data analysis: A design research approach to coding combining NVivo with traditional material methods. International Journal of Qualitative Methods, 17(1), 1609406918786362.

Malony, V. (2014, February 25). CBC closes Olympics on a ratings high. Playback Magazine. Retrieved from http://playbackonline.ca/2014/02/25/cbc-closes-olympics-on-a-ratingshigh/

Mankekar, P. (1999). Screening Culture, Viewing Politics: An Ethnography Of Television, Womanhood, And Nation In Postcolonial India. Duke University Press.

Markovits, A. S., Albertson, E. K., \& Albertson, E. K. (2012). Sportista: Female Fandom In The United States. Temple University Press.

Markula, P. (2003). The technologies of the self: Sport, feminism, and Foucault. Sociology of sport journal, 20(2), 87-107.

Markula, P., Grant, B. C., \& Denison, J. (2001). Qualitative research and aging and physical activity: Multiple ways of knowing. Journal of Aging and Physical Activity, 9(3), 245264.

Massumi, B. (2003). The archive of experience. In J. Brouwer \& A. Mulder (Eds.), Information is Alive (pp. 142-151). V2Publishing/NAI Publishers. 
McDonagh, E., \& Pappano, L. (2007). Playing With The Boys: Why Separate Is Not Equal In Sports. Oxford University Press.

McDonald, M. G. (2000). The marketing of the women's national basketball association and the making of postfeminism. International Review for the Sociology of Sport, 35(1), 35-47.

McDonald, M. G. (2012). Out of bounds plays: The Women's National Basketball Association and the neoliberal imaginings of sexuality. In D.L. Andrews \& M.L. Silk (Eds.), Sport and Neoliberalism: Politics, Consumption, and Culture (pp. 211-224). Temple University Press.

McDonald, M. G. (2015). Imagining neoliberal feminisms? Thinking critically about the US diplomacy campaign, 'Empowering women and girls through sports'. Sport in Society, 18(8), 909-922.

McFarlane, B. (1994). Proud Past, Bright Future: 100 Years of Canadian Women's Hockey. Toronto: Stoddart.

McGran, K. (2019, March 31). The Canadian Women's Hockey League is folding, board says business model 'isn't the right one'. The Toronto Star. Retrieved from https://www.thestar.com/sports/hockey/2019/03/31/canadian-womens-hockey-league-isfolding-board-says-business-model-is-financially-unsustainable.html

McGran, K. (2019, May 30). Is the NBA more popular than NHL in Canada? The Toronto Star. Retrieved from https:/www.thestar.com/sports/breakaway_blog/2019/05/30/is-the-nbamore-popular-than-nhl-in-canada-.html

McKay, J. (1993). "Marked men" and "Wanton women": The politics of naming sexual "Deviance" in sport. The Journal of Men's Studies, 2(1), 69-87. doi:10.3149/jms.0201.69

McKay, J., \& Rowe, D. (1997). Field of soaps: Rupert v. Kerry as masculine melodrama. Social Text, (50), 69-86.

McKay, J., \& Smith, P. (1995). Exonerating the hero: Frames \& narratives in media coverage of the OJ Simpson story. Media Information Australia, 75(1), 57-66.

McKay, J., Messner, M. A., \& Sabo, D. (2000). Masculinities, Gender Relations, And Sport (Vol. 11). Sage Publications.

McKeon, L. (2018, February 27). Unequal playing field: Olympics media coverage is still sexist. TVOntario. Retrieved from https://www.tvo.org/article/unequal-playing-field-olympicsmedia-coverage-is-still-sexist

McRobbie, A. (1978) Working Class Girls and the Culture of Femininity. In Women's Studies Group, Centre for Contemporary Cultural Studies (Ed.), Women Take Issue: Aspects of Women's Subordination. London: Hutchinson.

McRobbie, A. (2007). Top girls? Young women and the post-feminist sexual contract. Cultural Studies, 21(4-5), 718-737. 
McRobbie, A. (2009). The Aftermath Of Feminism: Gender, Culture And Social Change. Sage.

McVee, M.B., Dunsmore, K. \& Gavelek, J.R. (2005). Schema theory revisited. Review of Educational Research, 75, 531-566.

Meân, L. (2009). Making masculinity and framing femininity. In Hundley, H. L., \& Billings, A. C. (Eds.). Examining identity in sports media (pp. 65-86). Sage Publications.

Meân, L. (2012). Empowerment Through Sport?. In K. Toffoletti \& P. Mewett (Eds.), Sport And Its Female Fans (pp. 169-192). Routledge.

Meân, L. J. (2010). Making masculinity and framing femininity: FIFA, soccer, and world cup web sites. In Examining Identity in Sports Media. (pp. 65-86). SAGE Publications Inc..

Meehan, E. R. (2002). Gendering the commodity audience: Critical media research, feminism, and political economy. In Eileen R. Meehan \& Ellen Riordan (Eds.) Sex \& Money: Feminism And Political Economy In The Media (pp. 209-222). University of Minnesota Press.

Meese, J., \& Podkalicka, A. (2015). Practices of media sport: Everyday experience and audience innovation. Media International Australia, 155(1), 89-98.

Merton, R. K. (1987). The focussed interview and focus groups: Continuities and discontinuities. The Public Opinion Quarterly, 51(4), 550-566.

Merton, R.K. (1949) Social Theory and Social Structure. Glencoe, IL: Free Press.

Messner, M. A. (1988). Sports and male domination: The female athlete as contested ideological terrain. Sociology of Sport Journal, 5(3), 197-211.

Messner M (2011) Gender ideologies, youth sports and the production of soft essentialism. Sociology of Sport Journal 28(2), 151-170.

Messner, M. A. (2015). Male Athletes, Injuries, and Violence. Sociological Perspectives on Sport: The Games Outside the Games, 446.

Messner, M. A., Dunbar, M., \& Hunt, D. (2000). The televised sports manhood formula. Journal of Sport and Social Issues, 24(4), 380-394.

Messner, M. A., Duncan, M. C., \& Wachs, F. L. (1996). The gender of audience building: Televised coverage of women's and men's NCAA basketball. Sociological Inquiry, 66(4), 422-440.

Minnesota Whitecaps cry foul on exclusion from Canadian Women's Hockey League. (2012, January 18). Huffington Post. Retrieved from https://www.huffingtonpost.ca/2011/11/18/minnesota-whitecaps-cry-f_n_1102142.html

Mirtle, J. (2013, November 8). The great offside: How Canadian hockey is becoming a game strictly for the rich. The Globe and Mail. Retrieved from https://www.theglobeandmail.com/news/national/time-to-lead/the-great-offside-howcanadian-hockey-is-becoming-a-game-strictly-for-the-rich/article15349723/ 
Modleski, T. (1979). The search for tomorrow in today's soap operas. Film Quarterly, 33(1), 12.

Modleski, T. (1982). Loving With A Vengeance: Mass Produced Fantasies For Women. New York: Routledge, Chapman and Hall, Inc.

Mohorovich, M. (2018, February 27). Olympic Women's Hockey Doesn't Need to be Changed. Last World on Sports. Retrieved from https://lastwordonhockey.com/2018/02/21/olympic-womens-hockey-doesnt-needchanged/

More Than $85 \%$ Of Canada Tunes In For CBC/Radio-Canada's Coverage Of The Olympic Winter Games Pyeongchang 2018. (2018, February 27). [Press Release]. CBC Sports. Retrieved from https:/www.cbc.ca/mediacentre/press-release/more-than-85-of-canadatunes-in-for-cbc-radio-canadas-coverage-of-pyeongcha

More trouble for women's hockey as Buffalo Beauts owner hands franchise back to NWHL | CBC Sports. (2019, May 08). CBC Sports. Retrieved from https://www.cbc.ca/sports/hockey/nwhl-kim-pegula-buffalo-beauts-1.5128271

Morgan, D. L. (1996). Focus groups. Annual review of sociology, 22(1), 129-152.

Morgan, D. L. (1997). Focus Groups As Qualitative Research. (2nd ed.) Sage Publications.

Morgan, D. L. (2007). Paradigms lost and pragmatism regained: Methodological implications of combining qualitative and quantitative methods. Journal of Mixed Methods Research, $1(1), 48-76$.

Morley, D. (1980). The Nationwide Audience: Structure and Decoding. London: British Film Institute.

Morley, D. (1986). Family Television: Cultural Power And Domestic Leisure. Routledge.

Morley, D. (1992). Television, Audiences, And Cultural Studies. Routledge.

Morley, D. (1993). Active audience theory: pendulums and pitfalls. Journal of communication, 43(4), 13-19.

Morley, D. (2006). Unanswered questions in audience research. The Communication Review, $9(2), 101-121$.

Morley, D., \& Brunsdon, C. (1999). The Nationwide Television Studies. Routledge.

Morrison, D. E. (2001). The Search For A Method: Focus Groups And The Development Of Mass Communication Research. Indiana University Press.

Moseid, C. H., Myklebust, G., Fagerland, M. W., \& Bahr, R. (2019). The association between early specialization and performance level with injury and illness risk in youth elite athletes. Scandinavian Journal Of Medicine \& Science In Sports, 29(3), 460-468.

Mountjoy, M. (2019). 'Only by speaking out can we create lasting change': what can we learn from the Dr. Larry Nassar tragedy? British Journal of Sports Medicine, 53(1), 57-60. 
Muller, N. L. (1995). as the [sports] world turns: An analysis of the Montana-49er social drama. Journal of Sport \& Social Issues, 19(2), 157-179.

Mumford, L. S. (1995). Love And Ideology In The Afternoon: Soap Opera, Women, And Television Genre. Indiana University Press.

Murphy, M. (2017, November 22). Women's hockey leagues NWHL, CWHL set example for inclusivity in sports. Sporting News. Retrieved from https://www.sportingnews.com/ca/nhl/news/nwhl-cwhl-nhl-harrison-browne-laurendahm-kelsey-koelzer-anya-battaglino/1m17lgodt2aig14h8avfypnfg3

Murphy, M. (2017, October 20). NWHL releases salary details for 2017-18 season. The Ice Garden. Retrieved from https://www.theicegarden.com/2017/10/20/16508376/nwhlreleases-salary-details-for-2017-18-season-womens-hockey-pay-players-tierscompensation-nwhlpa

Murphy, M. (2018, September 05). Women's Hockey is Growing Strong. The Ice Garden. Retrieved from https://www.theicegarden.com/2018/9/5/17759722/growing-strongwomens-hockey-growing-popularity-growth-usa-pyeongchang-nwhl-cwhl

Nagoshi, J. L., \& Brzuzy, S. (2010). Transgender theory: Embodying research and practice. Affilia, 25(4), 431-443. doi:10.1177/0886109910384068.

Nafio. (2018, September 13). 2018-19 CWHL season ticket packages are on sale now! Retrieved from https://www.pensionplanpuppets.com/cwhl/2018/9/13/17856608/2018-19-cwhlseason-ticket-packages-on-sale-toronto-furies-markham-thunder-womens-hockey

Napoli, P. M. (2003). Audience Economics: Media Institutions And The Audience Marketplace. Columbia University Press.

Napoli, P. M. (2011). Audience Evolution: New Technologies And The Transformation Of Media Audiences. Columbia University Press.

Napoli, P. M. (2012). Audience evolution and the future of audience research. International Journal on Media Management, 14(2), 79-97

Neal, A. (2019, July 13). Yes, the WNBA Wage Gap Is a Real Thing. Grandstand Central. Retrieved from https://grandstandcentral.com/2018/sports/basketball/yes-the-wnba-wagegap-is-real/

Neale, J. (2016, April 23). NWHL threatened by alleged investor through leaked document. Yahoo Sports. Retrieved from https://ca.sports.yahoo.com/blogs/nhl-puck-daddy/nwhlthreatened-by-alleged-investor-through-leaked-document-055350338.html

Negra, D. (2009) What a Girl Wants? Fantasizing the Reclamation of Self in Postfeminism. Oxon: Routledge.

NHL's Canadiens, CWHL's Stars strike partnership to grow women's game. (2015, March 19). CTV News. Retrieved from https://montreal.ctvnews.ca/nhl-s-canadiens-cwhl-s-starsstrike-partnership-to-grow-women-s-game-1.2288634 
Nightingale, V. (1993). What's 'ethnographic' about ethnographic audience research? In G. Turner (Ed.), Nation, Culture, Text: Australian Cultural And Media Studies (pp. 164177). Routledge.

Noël, B. (2015, August 03). What Does the Launch of the NWHL Mean for the CWHL? Vice. Retrieved from https://www.vice.com/en_ca/article/bmqwq3/what-does-the-launch-ofthe-nwhl-mean-for-the-cwhl

Norman, L. (2010). Feeling second best: Elite women coaches' experiences. Sociology of Sport Journal, 27(1), 89-104.

Norton, W. (2009). Women On Ice: The Early Years Of Women's Hockey In Western Canada. Ronsdale Press.

NWHL cancels expansion plans, open to talk of new league. (2019, May 30). Sportsnet. Retrieved from https://www.sportsnet.ca/hockey/nhl/nwhl-cancels-expansion-plans-opentalk-new-league/

O'Connor, B., \& Boyle, R. (1993). Dallas with balls: televised sport, soap opera and male and female pleasures. Leisure Studies, 12(2), 107-119.

Oates, T. P. (2012). Representing the audience: The gendered politics of sport media. Feminist Media Studies, 12(4), 603-607.

Olympics Committee's policy on transsexual athletes at the 2004 Athens summer games. Body \& Society, 12, 75-102.

Ontario Ministry of Tourism, Culture and Sport. (2017). Advancing Opportunities for Women and Girls in Sport: Ontario's Action Plan. [Action Plan]. Retrieved from http://www.mtc.gov.on.ca/en/sport/women_sport_plan.shtml

Osborne, A. C., \& Coombs, D. S. (2013). Performative sport fandom: An approach to retheorizing sport fans. Sport in Society, 16(5), 672-681.

Osborne, A. C., \& Coombs, D. S. (2015). Female Fans of the NFL: Taking their Place in the Stands. Routledge.

Otterson, J. (2018, February 05). TV Ratings: Super Bowl LII Slips 7\% From 2017 to 103.4 Million Viewers. Variety. Retrieved from https:/variety.com/2018/tv/news/super-bowllii-ratings-1202687239/

Packer, C., Geh, D. J., Goulden, O. W., Jordan, A. M., Withers, G. K., Wagstaff, A. J., Bellwood, R.A., Binmore, C.L., \& Webster, C. L. (2014). No lasting legacy: no change in reporting of women's sports in the British print media with the London 2012 Olympics and Paralympics. Journal of Public Health, 37(1), 50-56.

Paechter, C. (2018). Rethinking the possibilities for hegemonic femininity: Exploring a Gramscian framework. Women's Studies International Forum, 68, 121-128. doi:10.1016/j.wsif.2018.03.005 
Parviainen, J. (2018). Insert name of openly gay hockey player here: Attitudes towards homosexuality among Canadian male major midget AAA ice hockey players. Sociology of Sport Journal, 35(4), 347. doi:10.1123/ssj.2017-0168

Patton, K. (2018, February 07). Canadians lukewarm about Olympics without NHL: Poll. Surrey Now. Retrieved from https://www.surreynowleader.com/sports/canadians-luke-warmabout-olympics-without-nhl-presence/

Pelak, C. F. (2002). Women's collective identity formation in sports: A case study from women's ice hockey. Gender \& Society, 16(1), 93-114.

Perez, A. (2019, May 20). Women's hockey stars form players association in bid to get sustainable pro league. USA Today. Retrieved from https://www.usatoday.com/story/sports/hockey/2019/05/20/womens-hockey-starsplayers-association/3742818002/

Peters, C. (2015, October 11). NWHL, first paid women's pro hockey league, drops puck on first season. CBC Sports. Retrieved from https:/www.cbssports.com/nhl/news/nwhl-firstpaid-womens-pro-hockey-league-drops-puck-on-first-season/

Pfister, G. (2016). Gender, gender equality and women's sport. In R. Lopez de D'Amico, T, Benn, \& G. Pfister. Women and Sport in Latin America (pp. 32-54). Routledge.

Phillips, R. J. (2018). An Inquiry into the Political Economy of Hockey Night in Canada: Critically Assessing Issues of Ownership, Advertising, and Gendered Audiences. Canadian Journal of Communication, 43(2).

Pieper, L. (2016). Sex Testing: Gender Policing In Women's Sports. University of Illinois Press.

Pierce, D. (2019, March 17). The Future of Sports Is Interactive, Immersive and Intense. Wall Street Journal. Retrieved from https://www.wsj.com/articles/the-future-of-sports-isinteractive-immersive-and-intense-11552827600

Pimental, T. (2018, March 10). Olympian Brigette Lacquette aims to inspire young Indigenous hockey players. APTN News. Retrieved from https://aptnnews.ca/2018/03/09/olympianbrigette-lacquette-aims-inspire-young-indigenous-hockey-players/

Pitter, R. (2006). Racialization and hockey in Canada: From personal troubles to a Canadian challenge. In Whitson, D., Gruneau, R. (Eds.), Artificial Ice: Hockey, Culture, And Commerce (pp. 123-142). University of Toronto Press.

Plantinga C.R. \& Smith G.M. (1999). Passionate Views: Film, Cognition, and Emotion. Johns Hopkins University Press.

Players 'shocked' as Canadian Women's Hockey League folds. (2019, March 31). City News. Retrieved from https://edmonton.citynews.ca/2019/03/31/canadian-womens-hockeyleague-folds/

Plaza, M., \& Boiché, J. (2017). Gender stereotypes, self, and sport dropout: a one-year prospective study in adolescents. Movement Sport Sciences, (2), 75-84. 
Poniatowski, K. (2011). "You're Not Allowed Bodychecking in Women's Hockey": Preserving Gendered and Nationalistic Hegemonies in the 2006 Olympic Ice Hockey Tournament. Women in Sport and Physical Activity Journal, 20(1), 39-52.

Poniatowski, K., \& Hardin, M. (2012). "The More Things Change, the More They...”: Commentary During Women's Ice Hockey at the Olympic Games. Mass Communication and Society, 15(4), 622-641.

Poniatowski, K., \& Whiteside, E. (2012). “Isn't He a Good Guy?”: Constructions of Whiteness in the 2006 Olympic Hockey Tournament. Howard Journal of Communications, 23(1), 116.

Pope, S. (2011). 'Like pulling down Durham Cathedral and building a brothel': Women as 'new consumer' fans?. International Review for the Sociology of Sport, 46(4), 471-487.

Power, P. (2013, April 28). Women's sports media group calls Don Cherry's comments 'sexist'. The Globe and Mail. Retrieved from https:/www.theglobeandmail.com/sports/womenssports-media-group-calls-don-cherrys-comments-sexist/article11600656/

Poynter, R. (2010). The Handbook Of Online And Social Media Research: Tools And Techniques For Market Researchers. John Wiley \& Sons.

Pratten, J. D. (2003). Professional wrestling - multi-million pound soap opera of sports entertainment. Management Research News, 26(5), 32-43.

Press, A. L. (1990). Class, gender and the female viewer: Women's responses to Dynasty. In M.E. Brown (Ed.), Television And Women's Culture: The Politics Of The Popular (pp. 144-157). Sage Publications.

Press, A. L. (2006). Audience research in the post-audience age: An introduction to Barker and Morley. The Communication Review, 9(2), 93-100.

Pringle, R. (2015). When the Pleasure Is Political: An Affective Analysis of Viewing the Olympics, In Pringle, R., Rinehart, R. E., \& Caudwell, J. (Eds.) Sport And The Social Significance Of Pleasure (pp. 116-145). Routledge. 116-145.

Probyn, E. (2005). Blush: Faces of Shame. University of Minnesota Press. Retrieved from http://www.jstor.org/stable/10.5749/j.ctttt6tjCopy

Pronger, B. (1999). Outta my endzone: Sport and the territorial anus. Journal of Sport and Social Issues, 23(4), 373-389.

Proteau, A. (2014, June 12). Gay female Olympic hockey players are increasingly comfortable coming out - and that's great. The Hockey News. Retrieved from https://thehockeynews.com/news/article/gay-female-olympic-hockey-players-areincreasingly-comfortable-coming-out-and-thats-great

Purdy, C. (2018, December 19). Deadly Humboldt Broncos bus crash chosen by Canadian media as News Story of the Year. CBC News. Retrieved from https://www.cbc.ca/news/canada/saskatoon/humboldt-crash-story-of-the-year-1.4951837 
Pyeongchang 2018: TSN National Broadcast Schedule. (2018, February 02). TSN. Retrieved from https://www.tsn.ca/pyeongchang-2018-tsn-national-broadcast-schedule-1.986877

Radway, J. (1984). Interpretive communities and variable literacies: The functions of romance reading. Daedalus, $113(3), 49-73$

Radway, J. A. (1984). Reading The Romance: Women, Patriarchy, And Popular Literature. University of North Carolina Press.

Ramirez, K. (2018, May 08). A History Of Women's Sports Media Coverage Progress \& How Much Further It Needs To Go. Girl Talk HQ. Retrieved from https://girltalkhq.com/ahistory-of-womens-sports-media-coverage-progress-how-much-further-it-needs-to-go/

Raney, A. A., \& Bryant, J. (2009). Handbook Of Sports And Media. Routledge.

Reid, P. A., \& Mason, D. S. (2015). 'Women can't skate that fast and shoot that hard!': The first women's world ice hockey championship, 1990. The International Journal of the History of Sport, 32(14), 1678-1696.

Reid, P. A., \& Mason, D. S. (2017). Sport signifiers and symbols: an ideographic analysis of the 1990 women's world ice hockey championship. Managing Sport and Leisure, 22(5), 374389.

Reslen, E. (2018, February 23). Jocelyne Larocque Removes Silver Medal After Loss. Yahoo News. Retrieved from https://ca.news.yahoo.com/jocelyne-larocque-removes-silvermedal-001504287.html

Reynolds, T. (2016, January 09). Canada's Kaillie Humphries drives first 4-woman bobsled against men in World Cup. Global News. Retrieved from https://globalnews.ca/news/2443729/humphries-drives-first-4-woman-bobsled-againstmen-in-world-cup/

Rich, A. (1980). Compulsory heterosexuality and lesbian existence. Signs: Journal Of Women In Culture And Society, 5(4), 631-660.

Ritter, L. A., \& Sue, V. M. (2007). Selecting a sample. New Directions for Evaluation, (115), 2328.

Robertson, G. (2018, February 23). Rules are rules: After shunning silver, Jocelyne Larocque ordered to wear medal. The Globe and Mail. Retrieved from https://www.theglobeandmail.com/sports/olympics/rules-trump-emotion-as-canadianwomens-hockey-player-ordered-to-wear-silver-medal/article38062105/

Roberts, M. L. (2002). True womanhood revisited. Journal of Women's History, 14(1), 150-155.

Robidoux, M. (2001). Men At Play: A Working Understanding Of Professional Hockey. McGillQueen's Press-MQUP.

Robidoux, M. A. (2002). Imagining a Canadian identity through sport: A historical interpretation of lacrosse and hockey. Journal of American Folklore, 115(456), 209-225. 
Robson, M. (2002). Problem-solving In Groups. Gower Publishing, Ltd..

Rodriguez, A. (2017, May 19). VP Roundtable: On Race and Representation in Women's Hockey. Victory Press. Retrieved from https:/victorypress.org/2017/05/19/vproundtable-on-race-and-representation-in-womens-hockey/

Rody-Mantha, B. (2018). The Stanley Cup Playoffs, by the numbers. Media In Canada. Retrieved from http://mediaincanada.com/2018/06/21/the-stanley-cup-playoffs-by-thenumbers/

Rogers, E. M. (1994). A History Of Communication Study: A Biographical Approach. The Free Press.

Rogers, E. M., \& Cartano, D. G. (1962). Methods of measuring opinion leadership. Public Opinion Quarterly, (26), 435-441.

Rorke, T., \& Copeland, A. (2017). Athletic disobedience: providing a context for analysis of Colin Kaepernick's protest. FairPlay, Revista de Filosofia, Ética y Derecho del Deporte, (10), 83-107.

Rosen, B. (2019, April 08). Female hockey players devastated after CWHL folds. Global News. Retrieved from https://globalnews.ca/news/5141928/female-hockey-players-react-cwhlfolds/

Rowe, D. (2004). Sport, Culture And The Media: The Unruly Trinity (2nd ed.) Open University Press.

Rowe, D. (2017). We're all transnational now: sport in dynamic sociocultural environments. Sport in Society, 20(10), 1470-1484.

Rubin, A. M. (2009). Uses-and-gratifications perspective on media effects. In J. Bryant, D. Zillman, \& M.B. Oliver (Eds.), Media effects: Advances in Theory and Research (pp. 181-200). Routledge.

Rubin, A. M., \& Bantz, C. R. (1987). Utility of videocassette recorders. American Behavioural Scientist, 30(5), 471-485.

Ruddock, A. (2011). Cultivation Analysis and Media Violence. In V. Nightingale (Ed.), The Handbook Of Media Audiences (pp. 340-359). John Wiley \& Sons.

Ruggiero, T. E. (2000). Uses and gratifications theory in the 21 st century. Mass Communication \& Society, 3(1), 3-37.

Rutherford, K. (2018). Can the CWHL teach China to love hockey? Sportsnet. Retrieved from https://www.sportsnet.ca/hockey/nhl/cwhl-china-hockey-krs-vanke-shenzhen/

Rutherford, K. (2019). Inside the frustrating, inspiring mess that's women's pro hockey. Sportsnet. Retrieved from https://www.sportsnet.ca/hockey/nhl/inside-cwhl-nwhl-messbig-read/ 
Rutherford, K. (n.d.). What Harrison Browne gives up to be the only man in women's hockey. Sportsnet. Retrieved from https://www.sportsnet.ca/hockey/nhl/harrison-browne-givesman-womens-hockey/

Ryan, D. (2017, October 10). The Bruins should follow the Devils' lead and invest in women's hockey. Vox Media. Retrieved from https://www.stanleycupofchowder.com/2017/10/10/16451020/nhl-investment-inwomens-hockey-bruins-womens-hockey-partnership-nwhl-cwhl-new-jersey-devils

Sadler, E. (2019, April 10). Commissioner Gary Bettman says NHL won't interfere with NWHL. Sportsnet. Retrieved from https://www.sportsnet.ca/hockey/nhl/commissioner-garybettman-says-nhl-wont-interfere-nwhl/

Safai, P. (2003). Healing the body in the "culture of risk": Examining the negotiation of treatment between sport medicine clinicians and injured athletes in Canadian intercollegiate sport. Sociology Of Sport Journal, 20(2), 127-146.

Said, E. W. (2003). Orientalism. Penguin

Saldana, J. (2011). Fundamentals of Qualitative Research (Understanding Qualitative Research) 1st Edition. Oxford University Press.

Sanderson, J., Weathers, M., Snedaker, K., \& Gramlich, K. (2017). "I was able to still do my job on the field and keep playing": an investigation of female and male athletes' experiences with (not) reporting concussions. Communication \& Sport, 5(3), 267-287.

Sargent, S. L., Zillmann, D., \& Weaver III, J. B. (1998). The gender gap in the enjoyment of televised sports. Journal of Sport and Social Issues, 22(1), 46-64.

Saussure, F. d. (2006). Writings In General Linguistics. Oxford University Press.

Savage, J., \& Yancey, C. (2008). The effects of media violence exposure on criminal aggression: A meta-analysis. Criminal Justice and Behaviour, 35(6), 772-791. doi: $10.1177 / 0093854808316487$

Scherer, J., \& Whitson, D. (2009). Public broadcasting, sport, and cultural citizenship: The future of sport on the Canadian broadcasting corporation? International Review for the Sociology of Sport, 44(2-3)/, 213-229. doi:10.1177/1012690209104798

Schirato, T. (2013). Sports Discourse. Bloomsbury Academic.

Schram, C. (2019, April 01). Women's Pro Hockey Must Find Its Way Forward After The Dissolution Of The CWHL. Forbes Magazine. Retrieved from https:/www.forbes.com/sites/carolschram/2019/04/01/womens-pro-hockey-must-findits-way-forward-after-the-dissolution-of-the-cwhl/\#40b35cd578c9

Schrøder, K. (2003). Researching Audiences. Edward Arnold Publishers.

Schrøder, K. C. (2000). Making sense of audience discourses: Towards a multidimensional model of mass media reception. European Journal of Cultural Studies, 3(2), 233-258. 
Schrøder, K. C. (2013). From dogmatism to open-mindedness? historical reflections on methods in audience reception research. The Communication Review, 16(1-2), 40-50.

Schrøder, K. C. (2019). Audience Reception Research in a Post-broadcasting Digital Age. Television \& New Media, 20(2), 155-169.

Sedgwick, E. K., \& Frank, A. (1995). Shame in the cybernetic fold: Reading Silvan Tomkins. Critical Inquiry, 21(2), 496.

Sehmby, D. S. (2002). Wrestling and popular culture. CLCWeb: Comparative Literature and Culture, 4(1), 5.

Seidler, V. (1991). Recreating Sexual Politics: Men, Feminism and Politics. Routledge.

Semerjian, T. Z., \& Cohen, J. H. (2006). "FTM means female to me": transgender athletes performing gender. Women in Sport and Physical Activity Journal, 15(2), 28-43.

Sherlock, J., \& Elsden, N. (2000). Going all the way': Female football fans and 'Ladette' culture in the UK. In M. Keech \& G. McFee (Eds.), Issues And Values In Sport And Leisure Cultures (pp. 123-140). Cardinals Publishers Group.

Sherry, E., Osborne, A., \& Nicholson, M. (2016). Images of sports women: A review. Sex Roles, 74(7-8), 299-309.

Sholle, D. (1991). Reading the audience, reading resistance: Prospects and problems. Journal of Film and Video, 43(1/2), 80-89.

Shouse, E. (Dec. 2005) "Feeling, Emotion, Affect," M/C Journal, 8(6). Retrieved from http://journal.media-culture.org.au/0512/03-shouse.php.

Slack, J. D., \& Allor, M. (1983). The political and epistemological constituents of critical communication research. Journal of Communication, 33(3), 208-218.

Sloan, L, R. (1989). The motive of sport fans. In J. H. Goldstein (Ed.), Sports, games and play: Social and psychological viewpoints (2nd ed.). Lawrence Erlbaum. 175-240

Smythe, Dallas. W. (1977). Communications: Blindspot of western Marxism. Canadian Journal of Political and Society Theory, 1(3), 1-28.

Sparks, R., \& Westgate, M. (2002). Broad-based and targeted sponsorship strategies in Canadian women's ice hockey. International Journal of Sports Marketing and Sponsorship, 4(1), 48-73.

Spencer, D. (2016, April 27). Cold relations between CWHL, NWHL get icier in trademark dispute. Toronto Sun. Retrieved from https://torontosun.com/2016/04/27/cold-relationsbetween-cwhl-nwhl-get-icier-in-trademark-dispute/wcm/f724a253-8501-4724-82d7$7 \mathrm{cc} 7 \mathrm{a} 9 \mathrm{e} 6 \mathrm{a} 0 \mathrm{ac}$

Spencer, D. (2017, March 31). Battle for equality continues for women's hockey | CBC Sports. CBC Sports. Retrieved from https://www.cbc.ca/sports/hockey/nhl/equality-womenshockey-1.4050849 
Spencer, D. (2019a, January 20). Merger, equality on players' minds at CWHL all-star game CBC Sports. CBC Sports. Retrieved from https:/www.cbc.ca/sports/hockey/cwhl-allstar-game-toronto-1.4986030

Spencer, D. (2019b, March 31). CWHL folding, female hockey players fearful for future of their game. Retrieved from https://nationalpost.com/pmn/sports-pmn/hockey-sports$\mathrm{pmn} /$ saying-business-model-is-financially-unsustainable-the-cwhl-is-folding

Spigel, L. (1992). Make Room For TV: Television And The Family Ideal In Postwar America. University of Chicago Press.

Standing Committee on Canadian Heritage. (2017). Women and Girls in Sport (Report). Retrieved from https://www.ourcommons.ca/DocumentViewer/en/42-1/CHPC/report-7

Stanton, F. N., \& Lazarsfeld, P. F. (Eds.). (1944). Radio Research: 1942-1943. Duell, Sloan and Pearce.

Steiss, A. (2018, May 2). IIHF - IIHF Disciplinary board decision. [Report]. Retrieved from https://www.iihf.com/en/events/2018/wm/news/2216/iihf-disciplinary-board-decision

Stevens, J. (2006). Women's hockey in Canada: After the "gold rush." In David Whitson \& Richard Gruneau (Eds.), Artificial Ice: Hockey, Culture, And Commerce. University of Toronto Press.

Stevens, J. (2018). Thirty years of “Going Global”: Women's International Hockey, Cultural Diplomacy, and the Pursuit of Excellence. In S. Irving, N. Baillargeon, \& C. Boissinot, (Eds.). Hockey: Challenging Canada's Game = Au-delà du Sport National (pp. 147-164). University of Ottawa Press.

Stevens, J., \& Holman, A. C. (2013). Rinkside: New scholarly studies on ice hockey and society. Sport in Society, 16(3), 251-253.

Stewart, K., \& Williams, M. (2005). Researching online populations: the use of online focus groups for social research. Qualitative Research, 5(4), 395-416.

Stinson, S. (2018, January 04). Making of a monster: How TSN transformed the world juniors from small-time curiosity into must-see TV. The National Post. Retrieved from https://nationalpost.com/sports/hockey/making-of-a-monster-how-tsn-transformed-theworld-juniors-from-small-time-curiosity-into-must-see-tv

Stoelting, S. (2011). Disclosure as an interaction: Why lesbian athletes disclose their sexual identities in intercollegiate sport. Journal of Homosexuality, 58(9), 1187-1210. doi:10.1080/00918369.2011.605731

Stone, A. (2015, March 18). The greatest women's hockey player in the world needs to find a job. USA Today. Retrieved from https://ftw.usatoday.com/2015/03/marie-philip-poulincanada-womens-ice-hockey-boston-university

Strauss, A. \& Corbin, J. (1990). Basics of Qualitative Research: Grounded Theory Procedures and Techniques. Sage. 
Strong, G. (2019, March 12). Canadian women's curlers seek equal payout at national events. CBC Sports. Retrieved from https://www.cbc.ca/sports/olympics/curling/curling-payoutmen-women-national-events-1.5053139

Sue, V. M., \& Ritter, L. A. (2012). Conducting Online Surveys. Sage.

Sugden, J. P., \& Tomlinson, A. (Eds.). (2012). Watching The Olympics: Politics, Power And Representation. Routledge.

Sveinson, K., \& Hoeber, L. (2015). Overlooking the obvious: An exploration of what it means to be a sport fan from a female perspective. Leisure Studies, 34(4), 405-419.

Sveinson, K., \& Hoeber, L. (2016). Female sport fans' experiences of marginalization and empowerment. Journal of Sport Management, 30(1), 8-21.

Swanson, D. L. (1977). The Uses And Misuses Of Uses And Gratifications Human Communication Research, 3(3), 214-221.

Swink, R. S. (2017). Lemony Liz and likable Leslie: audience understandings of feminism, comedy, and gender in women-led television comedies. Feminist Media Studies, 17(1), $14-28$.

Syal, R. (2018, March 07). Canadian women's sledge hockey team fights for the right to play. The Globe and Mail. Retrieved from https://www.theglobeandmail.com/sports/olympics/canadian-womens-sledge-hockeyparalympics/article38213587/

Sykes, H. (2006). Transsexual and transgender policies in sport. Women in Sport and Physical Activity Journal, 15(1), 3-13.

Szto, C. (2016). \# LOL at Multiculturalism: Reactions to Hockey Night in Canada Punjabi From the Twitterverse. Sociology of Sport Journal, 33(3), 208-218.

Szto, C., \& Gruneau, R. (2018). The Hockey Night in Canada Punjabi Broadcast: A Case Study in Ethnic Sports Media. In S. Irving, N. Baillargeon, \& C. Boissinot, (Eds.). Hockey: Challenging Canada's Game = Au-delà du Sport National (pp. 199 - 216). University of Ottawa Press.

Tagg, B. (2012). Transgender netballers: Ethical issues and lived realities. Sociology of Sport Journal, 29(2), 151-167.

Tamburrini, C., \& Tännsjö, T. (Eds.). (2005). Genetic technology and sport: ethical questions. Routledge.

Tarde, G. (1962). The Laws of Imitation (1903). New York: Henry, Holt and Co. Tarde, G, 5988.

Tasker, Y., \& Negra, D. (Eds.). (2007). Interrogating Postfeminism: Gender And The Politics Of Popular Culture. Duke University Press. 
Taylor, G., \& Thomass, B. (2017). Sports rights and public service media/public broadcasting: Case studies on economic and political implications. International Communication Gazette, 79(2), 111-119.

Taylor, K. R. (2018, March 25). Women's Sports Aren't About Bake Sales and Role Models. The Walrus. Retrieved from https://thewalrus.ca/womens-sports-arent-about-bake-sales-androle-models/

Tervo, M. (2001). Nationalism, sports and gender in Finnish sports journalism in the early twentieth century. Gender, Place and Culture: A Journal of Feminist Geography, 8(4), 357-373.

The Canadian Women's Hockey League to Discontinue Operations. (2019, March 31).[Press Release] Retrieved from http://www.thecwhl.com/the-canadian-womens-hockey-leagueto-discontinue-operations

Theberge, N. (1995). Gender, sport, and the construction of community: A case study from women's ice hockey. Sociology of Sport Journal, 12(4), 389-402.

Theberge, N. (1997). "It's Part Of The Game" Physicality and the Production of Gender in Women's Hockey. Gender \& Society, 11(1), 69-87.

Theberge, N. (1998). "Same Sport, Different Gender” A Consideration of Binary Gender Logic and the Sport Continuum in the Case of Ice Hockey. Journal of Sport and Social Issues, 22(2), 183-198.

Theberge, N. (2000). Higher Goals: Women's Ice Hockey And The Politics Of Gender. SUNY Press.

Theberge, N. (2002). Challenging the gendered space of sport: Women's ice hockey and the struggle for legitimacy. In S. Scraton \& A. Flintoff, Gender and Sport: A Reader (pp. 292-302. Routledge.

Theodoropoulou, V. (2018). Takings Sides, Social Identity, and Sport Audiences. In M.A. Click \& S. Scott (Eds.), The Routledge Companion to Media Fandom (pp. 221-229). Routledge.

TheUndefeated. (2016, December 19). Serena Williams sits down with Common to talk about race and identity. The Undefeated. Retrieved from https://theundefeated.com/features/serena-williams-sits-down-with-common-to-talkabout-race-and-identity/

Thing, L. F. (2001). The female warrior: Meanings of play-aggressive emotions in sport. International Review for the Sociology of Sport, 36(3), 275-288.

Thorpe, H. (2014). Transnational Mobilities In Action Sport Cultures. Springer.

Thorpe, H., \& Rinehart, R. (2010). Alternative sport and affect: Non-representational theory examined. Sport in Society, 13(7-8), 1268-1291. 
Thrift, N. (2008). Non-representational Theory: Space, Politics, Affect. Routledge.

Toepoel, V. (2015). Doing Surveys Online. Sage.

Toffoletti, K. (2017). Sexy women sports fans: Femininity, sexuality, and the global sport spectacle. Feminist Media Studies, 17(3), 457-472.

Toffoletti, K. (2017). Women Sport Fans: Identification, Participation, Representation. Routledge.

Toffoletti, K., \& Mewett, P. (Eds.). (2012). Sport And Its Female Fans (Vol. 17). Routledge.

Tolson, A. (1977). The Limits Of Masculinity: Male Identity And The Liberated Woman. Harper \& Row.

Travers, A. (2006). Queering sport: Lesbian softball leagues and the transgender challenge. International Review for the Sociology of Sport, 41(3-4), 431-446.

Travers, A. (2008). The sport nexus and gender injustice. Studies in Social Justice, 2, 79-101.

Travers, A., \& Deri, J. (2011). Transgender inclusion and the changing face of lesbian softball leagues. International review for the sociology of sport, 46(4), 488-507.

Tudor, A. (1998). Sports reporting: Race, difference and identity. In K. Brants, J. Hermes, \& L.V. Zoonan (Eds.), The Media In Question: Popular Cultures And Public Interests. Sage. 147-156.

Smith, L. T. (2012). Decolonizing Methodologies: Research And Indigenous Peoples (Second ed.) Zed Books.

UNESCO calls for fairer media coverage of sportswomen. (2018, February 8). [Press Release]. Retrieved from https://en.unesco.org/news/unesco-calls-fairer-media-coveragesportswomen

Vered, K. O., \& Humphreys, S. (2014). Postfeminist inflections in television studies. Continuum, 28(2), 155-163. doi:10.1080/10304312.2014.888037

Veri, M. J. (1999). Homophobic discourse surrounding the female athlete. Quest, 51(4), 355-368.

Vertinsky, P. (1994). Women, sport, and exercise in the 19th century. Women and sport: Interdisciplinary perspectives, 63-82.

Vincent, J., \& Crossman, J. (2012). "Patriots at Play": Analysis of newspaper coverage of the gold medal contenders in men's and women's ice hockey at the 2010 Winter Olympic Games. International Journal of Sport Communication, 5(1), 87-108.

Vincent, J., \& Crossman, J. (2015). "our game our gold": Newspaper narratives about the Canadian men's ice hockey team at the 2010 winter Olympic games. Journal of Sport Behaviour, 38(1), 97. 
Vincent, J., Kian, E. M., Pedersen, P. M., Kuntz, A., \& Hill, J. S. (2010). England expects: English newspapers' narratives about the English football team in the 2006 World Cup. International Review for the Sociology of Sport, 45(2), 199-223.

Vlessing, E. (2014, February 25). TV Ratings: CBC Wins With Canada's Hockey Gold. Hollywood Reporter. Retrieved from https://www.hollywoodreporter.com/news/tvratings-cbc-wins-canadas-682643

Von der Lippe, G. (2002). Media image: Sport, gender and national identities in five European countries. International review for the sociology of sport, 37(3-4), 371-395.

Wagner, D. (2019, May 30). It's likely that more Canadians will watch the NBA Finals than the Stanley Cup Final. Vancouver Courier. Retrieved from https://www.vancourier.com/pass-it-to-bulis/it-s-likely-that-more-canadians-will-watchthe-nba-finals-than-the-stanley-cup-final-1.23839083

Waldron, J. J. (2016). It's complicated: Negotiations and complexities of being a lesbian in sport. Sex Roles, 74(7-8), 335-346.

Walsh, I., Holton, J. A., Bailyn, L., Fernandez, W., Levina, N., \& Glaser, B. (2015). What grounded theory is... a critically reflective conversation among scholars. Organizational Research Methods, 18(4), 581-599.

Ward, L., \& Strashin, J. (2019, May 07). Intimidation, verbal abuse of Canada's elite athletes are not uncommon, study finds. Retrieved from $C B C$ News. https://www.cbc.ca/news/investigates/elite-athletes-abuse-1.5125147

Warner, W. L., \& Henry, W. E. (1948). The radio daytime serial: a symbolic analysis. Genetic Psychology Monographs.

Warr, D. J. (2005). “It was fun... but we don't usually talk about these things": analyzing sociable interaction in focus groups. Qualitative inquiry, 11(2), 200-225.

Watson, S. D. (2017). Everyday nationalism and international hockey: contesting Canadian national identity. Nations and Nationalism, 23(2), 289-308.

Wawrow, J. (2016, April 28). National Women's Hockey League sued by former executive. Associated Press. Retrieved from https://apnews.com/a535a1c872664b969bbe74fab6a309b8

We help our members understand the media habits of Canadians. (n.d.). Retrieved from http://en.numeris.ca/about-us/overview

Weaving, C., \& Roberts, S. (2012). Checking in: an analysis of the (lack of) bodychecking in women's ice hockey. Research Quarterly For Exercise And Sport, 83(3), 470-478.

Webster, J. G., Phalen, P. F., \& Lichty, L. W. (2006). Ratings Analysis: The Theory And Practice Of Audience Research (3rd ed.) L. Erlbaum Associates. 
Weir, P. L., Smith, K. L., Paterson, C., \& Horton, S. (2010). Canadian women's ice hockey: Evidence of a relative age effect. Talent Development \& Excellence, 2(2), 209-217.

Welter, B. (1966). The cult of true womanhood: 1820-1860. American Quarterly, 18(2), 151174.

Wenner, L. A. (Ed.). (1998). MediaSport. Psychology Press.

Wenner, L. A., \& Billings, A. C. (Eds.). (2017). Sport, media and mega-events. Taylor \& Francis.

Wenner, L. A., \& Gantz, W. (1989). The audience experience with sports on television. In L. Wenner (Ed.), Media, Sports, \& Society, (pp. 241-269). Sage Publications.

Wensing, E. H., \& Bruce, T. (2003). Bending the rules: Media representations of gender during an international sporting event. International review for the sociology of sport, 38(4), 387-396.

Whannel, G. (1992). Fields In Vision: Television Sport And Cultural Transformation. Routledge.

Whannel, G. (2002). Reading the sports media audience. In L. Wenner (Ed.), MediaSport (pp. 235-240). Routledge.

Whannel, G. (2009). Television and the transformation of sport. The Annals of the American Academy of Political and Social Science, 625(1), 205-218.

doi:10.1177/0002716209339144

Whannel, G., \& Horne, J. (2016). Understanding the Olympics. Routledge.

Wharnsby, T. (2019, March 23). Time is now for 1 women's hockey league - with assist from NHL. CBC Sports. Retrieved from https://www.cbc.ca/sports/hockey/nhl/tim-wharnsbyclarkson-cup-united-womens-league-1.5069151

Wheaton, B., \& Thorpe, H. (2018). Action sports, the Olympic Games, and the opportunities and challenges for gender equity: The cases of surfing and skateboarding. Journal of Sport and Social Issues, 42(5), 315-342.

Wheedon, C. (1997). Feminist Practice And Poststructural Theory. Malden, MA: Blackwell.

Whiteside, E., \& Hardin, M. (2011). Women (not) watching women: Leisure time, television, and implications for televised coverage of women's sports. Communication, Culture \& Critique, 4(2), 122-143.

Wickenheiser, H. [@wick_22]. (2019, March 31). Can’t help seeing today’s \#cwhl news as a positive step in the long run for women's hockey. One step back, two steps forward perhaps? [Tweet]. //twitter.com/wick_22/status/1112449176577757184?lang=en

Wickenheiser, H. (2018). Skating in the drainage ditches. In S. Irving, N. Baillargeon, \& C. Boissinot, (Eds.). Hockey: Challenging Canada's Game = Au-delà du Sport National (pp. 42-43). University of Ottawa Press. 
Williams, L. (1989). Fetishism and the visual pleasure of hard core: Marx, Freud, and the "money shot". Quarterly Review of Film \& Video, 11(2), 23-42.

Williams, R. (1974). Communications as cultural science. Journal of Communication, 24(3), 1725.

Willis, P. (1982). Women in sport in ideology. In J. Hargreaves (Ed.) Sport, Culture and Ideology, (pp. 117-135). Routledge \& Keegan Paul.

Wingrove, J. (2018, April 30). Jersey girls: Women lead the way in hockey's growth. The Globe and Mail. Retrieved from https://www.theglobeandmail.com/sports/jersey-girls-womenlead-the-way-in-hockeys-growth/article562294/

WNBA players opt out of CBA, can start negotiating sooner. (2018, December 12). The New York Times. Retrieved from https://www.nydailynews.com/sports/basketball/ny-sportswnba-cba-negotiating-20181101-story.html

Wong, J. (2019, April 16). CBC revives Battle of the Blades skate competition show | CBC News. Retrieved from https://www.cbc.ca/news/entertainment/botb-returns-1.5098953

Wood, H. (2007). The mediated conversational floor: an interactive approach to audience reception analysis. Media, Culture \& Society, 29(1), 75-103.

Wrench, J. S., Thomas-Maddox, C., Richmond, V. P., \& McCroskey, J. C. (2008). Quantitative Research Methods For Communication: A Hands-on Approach. Oxford University Press, Inc..

Wright, J., \& Clarke, G. (1999). Sport, the media and the construction of compulsory heterosexuality: A case study of women's rugby union. International Review For The Sociology Of Sport, 34(3), 227-243.

Wright, K. B. (2005). Researching Internet-based populations: Advantages and disadvantages of online survey research, online questionnaire authoring software packages, and web survey services. Journal Of Computer-mediated Communication, 10(3), JCMC1034.

Yiamouyiannis, A., \& Osborne, B. (2012). Addressing gender inequities in collegiate sport: Examining female leadership representation within NCAA sport governance. Sage Open, 2(2), 2158244012449340.

Young, K., White, P., \& McTeer, W. (1994). Body talk: Male athletes reflect on sport, injury, and pain. Sociology Of Sport Journal, 11(2), 175-194.

Young, L. (2018, February 22). Canada's Jocelyne Larocque under fire for taking off her silver medal in Olympic women's hockey. Global News. Retrieved from https://globalnews.ca/news/4040772/2018-winter-olympics-women-hockey-silverjocelyne-larocque/

Young, M. (1999). The social construction of tourist places. Australian Geographer, 30(3), 373389. 
Yuval-Davis, N. (2006). Intersectionality and feminist politics. European journal of women's studies, 13(3), 193-209.

Zelkovich, C. (2010, March 02). Gold-medal hockey game watched by record 16.6 million. The Toronto Star. Retrieved from https://www.thestar.com/sports/olympics/2010/03/01/goldmedal_hockey_game_watched _by_record_166_million.html

Zheng, J., \& Mason, D. S. (2018). Sport, Information, and the Attention Economy. In Brand Platform in the Professional Sport Industry (pp. 1-18). Palgrave Pivot, Cham. 